\title{
Skyrmion generation and perpendicular magnetic anisotropy modification in Heusler alloy ultrathin films
}

by

Yao Zhang

\author{
A thesis \\ submitted to the Victoria University of Wellington \\ in fulfilment of the \\ requirements for the degree of \\ Doctor of Philosophy \\ in Physics.
}

Victoria University of Wellington

2021 



\section{Acknowledgement}

I firstly would like to thank my supervisor, Dr. Simon Granville, for taking me to Wellington, the coolest little capital in the world, and for giving me the chance to pursue the PhD with a lot of research resources and support. He has a wealth of knowledge and always patiently explain it to me. I really enjoy to discuss science with him. Every time I have a new idea, I cannot wait to discuss with him. He is always excited and interested and gives me a lot of suggestions even though some of ideas are not related to my PhD project. I think Simon definitely knows creativity can not be forced because he always asks me to have a good rest and enjoy the life in this amazing country. I feel very sorry I push him a bit heavy sometimes so that he has to work at weekend. I have learned an enormous amount from Simon no matter science and life.

I also would like to thank Dr. Guy Dubuis for him invaluable advice and comments on my PhD project and publications. He teaches me a lot about engineering science which is the weakness for me who only focuses on fundamental physics. Guy spends a lot of time on managing equipment. Without that, my research project cannot go such smooth. By the way, Guy's pear pie is scrumptious.

I would like to thank A/Prof. Ben Ruck, my second supervisor, for his constructive suggestions. Ben looks serious, but actually has an excellent sense of humor though sometimes I cannot understand due to the language and culture. I also would like to thank people from his group for interesting discussions. It is very luck there are some of people study magnetism and spintronics in this small community.

I would like to thank Dr. Colin Doyle for the XPS measurement. The LEGO in his lab is so impressive. Many thanks to Tane Butler, Dr. Shen Chong and Andrew Best for their help for magnetron sputtering, laser writer, PPMS and SQUID. I am also very grateful to the collaborators in 
Monash University, Beihang University, University of New South Wales and Kyoto University. I have to thank all members of Robinson Research Institute, for the friendly environment they offered me, and the muffin and scone for Monday meeting. Heartfelt thanks go to my officemates: David Uhrig and Oscar Bjareborn. I will definitely miss the time we study together in this super warm office.

Finally, I would like to thank my dad, mum, brother and sister for all their support. You are the best family I could have asked for. I am happy my parents did not ask me "when can you graduate?" and "when can you publish paper?" such annoying questions. A special thanks to my wife, Lingping Liao, for every her dedication during these years. I feel selfish that she resigned and came to here, but we do have a memory time during these years. I remember we travelled $3000 \mathrm{~km}$ around New Zealand during Christmas holiday. The trip was so funny and we did not realize that we run out the memory at the end of the trip. Life must be better in the future. A particular thanks to my son, Hengyu Zhang, for bringing so much fun to my life. I am very happy you enjoy having a bush walk and camping with mum and I at weekend and holiday. I still remember you asked me which mountain we are going to this weekend. Also, I think we need a big tent for next journey, and do not forget to take your dinosaur pajamas and whistle. 


\begin{abstract}
This thesis explores the magnetic properties of Heusler alloy thin films for spintronics applications. The skyrmion generation under different external stimulation was focused in $\mathrm{MgO} / \mathrm{Mn}_{2} \mathrm{CoAl} / \mathrm{Pd}$ ultrathin films and the anomalous Hall effect of $\mathrm{Co}_{2} \mathrm{MnGa}$ thin films.

The perpendicularly magnetized $\mathrm{MgO} / \mathrm{Mn}_{2} \mathrm{CoAl} / \mathrm{Pd}$ ultrathin films were firstly optimised by changing the thickness of Pd layer so that the effective magnetic anisotropy can be tuned and various magnetic textures can be obtained for different purposes. Based on the understanding of Pd dependence of magnetic properties, The skyrmion generation by applying an in-plane magnetic field was investigated to fine tune the effective magnetic anisotropy. Further the fractal analysis was used to describe the evolution of the magnetic states and categorise the formation of skyrmions. Then skyrmion generation by ionic liquid gating has been investigated in this trilayer. Both non-volatile and volatile skyrmions can be generated by applying a range of voltage sequences. The potential mechanisms, magneto-ionic and electrostatic charge effects, have been discussed as well.

Finally, the thickness dependence of $\mathrm{Co}_{2} \mathrm{MnGa}$ thin films was studied. This material can be used as a spin-orbit generator for manipulating skyrmions. A large anomalous Hall angle (AHA) was demonstrated in $\mathrm{Co}_{2} \mathrm{MnGa}$ thin films (20 - $50 \mathrm{~nm}$ ) showing a AHA $11.4 \%$ at low temperature and $\sim 9.7 \%$ at room temperature, which can be ascribed to the nontrivial topology of the band structure with large intrinsic Berry curvature. However, the anomalous Hall angle decreases significantly with thicknesses below $20 \mathrm{~nm}$, which band structure calculations confirm is due to the reduction of the majority spin contribution to the Berry curvature.
\end{abstract}




\section{Contents}

1 Introduction and theory 1

1.1 Overview . . . . . . . . . . . . . . . . 1

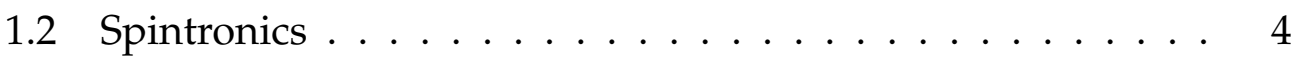

1.3 Origin of magnetism $\ldots \ldots \ldots \ldots$

1.4 Ferromagnetism . . . . . . . . . . . . . . . 10

1.5 Magnetic interactions, anisotropies and energy . . . . . . 12

1.5.1 Exchange interaction . . . . . . . . . . . . . . 12

1.5 .2 Zeeman energy . . . . . . . . . . . . . . . . 13

1.5.3 Magnetic dipolar anisotropy $\ldots \ldots \ldots$. . . . . . 13

1.5.4 Magnetocrystalline anisotropy . . . . . . . . . . . 14

1.5.5 Perpendicular magnetic anisotropy . . . . . . . . . 15

1.5.6 Dzyaloshinskii-Moriya interaction . . . . . . . . . 16

1.6 Magnetic spin textures . . . . . . . . . . . . . . . 17

1.6.1 Magnetic domains . . . . . . . . . . . . . . . 17

1.6 .2 Domain walls . . . . . . . . . . . . . . . . . . 18

1.6.3 Chiral domain walls . . . . . . . . . . . . . . 20

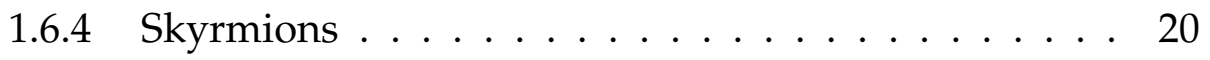

1.7 Heusler alloys . . . . . . . . . . . . . . . . . . . . 23

1.7.1 Crystal structure . . . . . . . . . . . . . . . . . 24

1.7.2 Half-metal and magnetism . . . . . . . . . . . . 25

1.7.3 Spin gapless semiconductors, $\mathrm{Mn}_{2} \mathrm{CoAl} \ldots \ldots . .28$

1.7.4 Magnetic Weyl semimetals, $\mathrm{Co}_{2} \mathrm{MnGa} \ldots \ldots \ldots . .28$ 
1.7.5 Skyrmions in Heusler alloys . . . . . . . . . . . . . 31

2 Methodology 33

2.1 Thin film growth $\ldots \ldots \ldots \ldots \ldots$

2.1 .1 Substrate cleaning . . . . . . . . . . . . 33

2.1.2 Thin film deposition . . . . . . . . . . . . . . 34

2.2 Device fabrication . . . . . . . . . . . . . . 36

2.2.1 Laser writer lithography . . . . . . . . . . . . . 37

2.2 .2 Ion milling . . . . . . . . . . . . . . . . . 38

2.3 Ionic liquid gating . . . . . . . . . . . . . . . . . . . . 39

2.4 Structure characterization . . . . . . . . . . . . . 40

2.4.1 X-ray diffraction and X-ray reflectivity . . . . . . . . 40

2.4.2 Scanning electron microscopy and energy-dispersive X-ray spectroscopy . . . . . . . . . . . . . . 42

2.4.3 Atomic force microscopy . . . . . . . . . . . . . . 43

2.4.4 X-ray photoelectron spectroscopy _. . . . . . . . 44

2.5 Electrical and magnetic characterization . . . . . . . . . . 45

2.5.1 Physical properties measurement system . . . . . . 46

2.5.2 Superconducting quantum interference device magnetometer . . . . . . . . . . . . . . . . . 49

2.5.3 Magneto-optical Kerr effect microscope . . . . . . . . 49

3 Thickness dependence of perpendicular magnetic anisotropy in $\mathrm{Mn}_{2}$ CoAl ultrathin films

3.1 Introduction . . . . . . . . . . . . . . . . . . . 59

3.2 Results . . . . . . . . . . . . . . . 60

3.2.1 Characterization of thick $\mathrm{Mn}_{2} \mathrm{CoAl} \ldots \ldots$. . . . . 60

3.2.2 Characterization of trilayers . . . . . . . . . . . 62

3.2.3 Thickness dependence of $\mathrm{Pd} \ldots$. . . . . . . . . . 63

3.3 Discussion and conclusions . . . . . . . . . . . . . . 65 
4 Skyrmions generated by in-plane magnetic field in $\mathrm{Mn}_{2} \mathrm{CoAl}$ ultrathin films $\quad 67$

4.1 Introduction . . . . . . . . . . . . . . . . 67

4.2 Results . . . . . . . . . . . . . . . . . . 68

4.2.1 Magnetization and chiral domain wall . . . . . . . . 68

4.2.2 Skyrmion generation assisted by an in-plane field . . 70

4.2.3 DMI calculation . . . . . . . . . . . . . . . . . . 74

4.2.4 Fractal analysis and magnetic phase diagram . . . . . 76

4.2.5 Further analysis of skyrmions formed from the inplane field . . . . . . . . . . . . . . . . 79

4.3 Discussion and conclusions . . . . . . . . . . . . . . 81

5 Skyrmions generated by ionic liquid gating in $\mathrm{Mn}_{2} \mathrm{CoAl}$ ultrathin films

5.1 Introduction . . . . . . . . . . . . . . . . . 83

5.2 Results . . . . . . . . . . . . . . . . 85

5.2.1 Non-volatile skyrmion generation by ILG . . . . . . . 85

5.2.2 Non-volatile skyrmion generation in intermediate PMA

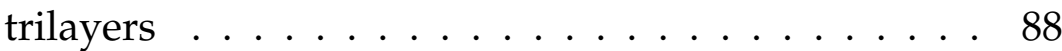

5.2.3 Volatile skyrmion generation by ionic liquid gating . 90

5.2 .4 Surface analysis . . . . . . . . . . . . . . . . . 92

5.3 Discussion and conclusions . . . . . . . . . . . . . . 94

6 Large anomalous Hall angle of $\mathrm{Co}_{2} \mathrm{MnGa} \quad 99$

6.1 Introduction . . . . . . . . . . . . . . . . . . . . 99

6.2 Results . . . . . . . . . . . . . . . . . . . . . 101

6.2.1 Sample preparation . . . . . . . . . . . . . . . . . . 101

6.2.2 Structure Characterization . . . . . . . . . . . . . . . . . 101

6.2.3 Magnetotransport properties . . . . . . . . . . . . 102

6.2.4 Evolution of Berry curvature and surface band structure . . . . . . . . . . . . . . . . . 110

6.3 Discussion and conclusions . . . . . . . . . . . . . . . . . 113 
7 Summary and outlook 117

7.1 Summary . . . . . . . . . . . . . . . . 117

7.2 Outlook . . . . . . . . . . . . . . . . . . 119

$\begin{array}{ll}\text { List of Publications } & 146\end{array}$

$\begin{array}{ll}\text { List of Figures } & 146\end{array}$

$\begin{array}{lr}\text { List of Abbreviations } & 159\end{array}$ 


\section{Chapter 1}

\section{Introduction and theory}

In this chapter, firstly, an overview for the whole thesis is gave (section 1.1). Then, the history of spintronics is briefly introduced (section 1.2). After that, in section 1.3 and 1.4, the principles of magnetism and ferromagnetism are given. The magnetic interactions, anisotropies and energy in ferromagnetic thin films (section 1.5) are discussed. Based on the balance of these energies, ferromagnetic thin films show various magnetic spin textures (section 1.6). In the section 1.7, the materials, Heusler alloys, which have been studied in this work are introduced.

\subsection{Overview}

The static or dynamic random-access memory (RAM) based on semiconductor technology enables fast read and write operations. However, these RAMs require power to retain the stored information due to their volatile behaviour meaning that the data will be lost once the power is switched off [1]. Spintronics is a research field manipulating the spin of electron for the next-generation nanoelectronic devices to increase their energy efficiency, memory and processing capabilities [2]. Over the past decade, magnetoresistive random-access memory (MRAM) has been developed to replace the static or dynamic RAM [3]. This memory technology is a type 
of non-volatile RAM where information is stored as the magnetization of the ferromagnetic layer in magnetic tunnel junction (MTJ) unit cells. There is no requirement to retain the stored information by keeping the power on so that it is much more efficient. Moreover, MRAM can operate at higher temperatures $\left(125^{\circ} \mathrm{C}\right)$ and radiation $\left(84 \mathrm{MeV} \mathrm{cm}^{2} / \mathrm{mg}\right)$ [4], making it even more useful. To improve the high storage density of MRAM, the size of MTJ needs to be reduced which also decreases the thermal stability. Materials with perpendicular magnetic anisotropy (PMA) possess high thermal stability which makes them suitable for spintronics applications and attracts attention from all around the world [5].

On the other hand, instead of storing information by uniform magnetization, skyrmions, a whirling spin textures with nanoscale dimension, can be used as information carriers in skyrmion-based racetrack memories [6]. In thin films, skyrmions usually can be observed in the system with PMA and Dzyaloshinskii-Moriya interaction (DMI) [7]. Currently, researchers focus on generating skyrmions in the traditional ferromagnetic materials, like $\mathrm{Fe}, \mathrm{Co}, \mathrm{CoFeB}$, in multilayers sandwiched by the $\mathrm{MgO}$ and heavy metal thin films [8,9]. In the last decade, different materials have been investigated to satisfy the requirement of PMA and skyrmions. Heusler alloys have low damping constant, high spin polarization and tunable properties which make them ideal for spintronics applications [10]. Very recently magnetic Weyl semimetals with emerging phenomena have been found in Heusler alloys [11,12]. This material shows a very large anomalous Hall angle which can be used a spin torque generator to drive skyrmion motion.

Though ferromagnet/heavy metal multilayers show great advantages for skyrmion-based applications, skyrmions only appear in a very narrow range of thicknesses, so it can require an extensive experimental campaign to produce multiple samples in attempts to find that narrow range. This is a challenge for sample preparation and is curtailing possible applications.

To realize the real skyrmion-based applications, methods are needed to 
manipulate skyrmions. The anomalous spin-orbit torque induced by anomalous Hall effect in ferromagnetic materials can be used to drive skyrmion motion. To improve the efficiency of this torque, it is important to find and study a ferromagnetic thin films with large anomalous Hall effect

Motivated by these challenges for fundamental research and practical application of skyrmions, two methods had been employed to generate skyrmion in $\mathrm{MgO} / \mathrm{Mn}_{2} \mathrm{CoAl} / \mathrm{Pd}$ ultrathin films and the $\mathrm{Co}_{2} \mathrm{MnGa}$ thin films with large anomalous Hall effect had been studied. This thesis is organized as follows:

Chapter 1: The general background is provided so that the reader can follow the study discussed in this thesis.

Chapter 2: The experimental techniques used to grow, design and characterize the samples are introduced.

Chapter 3: The Pd thickness dependence of perpendicular magnetic anisotropy and magnetic texture in $\mathrm{MgO} / \mathrm{Mn}_{2} \mathrm{CoAl} / \mathrm{Pd}$ ultrathin films has been studied. Therefore, the trilayers can be designed for different purposes.

Chapter 4: A powerful method that applying an in-plane magnetic field generates skyrmions is established in $\mathrm{MgO} / \mathrm{Mn}_{2} \mathrm{CoAl} / \mathrm{Pd}$ system. The fractal analysis is used to study the evolution of skyrmions as a useful tool.

Chapter 5: The method, ionic liquid gating, is used to generate skyrmions in $\mathrm{MgO} / \mathrm{Mn}_{2} \mathrm{CoAl} / \mathrm{Pd}$ films by applying a range of voltage sequences. The ionic liquid gating has been demonstrated a versatile method to engineer both non-volatile and volatile skyrmions by tuning the magnetic characteristics of films to the regimes where they can exist.

Chapter 6: The ferromagnetic Weyl semimetal $\mathrm{Co}_{2} \mathrm{MnGa}$ thin films show a large anomalous Hall angle which can be ascribed to the nontrivial topology of the band structure with large intrinsic Berry curvature. However, the anomalous Hall angle decreases significantly with thicknesses below $20 \mathrm{~nm}$ due to the reduction of the majority spin contribution to the 
Berry curvature.

Chapter 7: the conclusions and some interesting perspectives are addressed.

\subsection{Spintronics}

Spintronics, or spin electronics, is a field of research to realize the ultralow consumption and ultrahigh density nanoelectronics devices for magnetic memory, logic, and computing [2]. Instead of using the charge degree of freedom in microelectronics, spintronics devices manipulate the spin of the electron.

The development of spintronics was triggered by the discovery of giant magnetoresistance (GMR), in Fe/Cr multilayers by Albert Fert and Peter Grünberg independently in 1988 [13, 14]. In 2007, both of them were awarded the Nobel Prize in physics. The GMR effect is the change of resistance of thin films, such as $[\mathrm{Fe} / \mathrm{Cr} / \mathrm{Fe}]_{n}$ and $[\mathrm{Co} / \mathrm{Cu} / \mathrm{Co}]_{n}$ multilayers, in response to an external magnetic field. The multilayer exhibits low (high) resistance when the magnetization of ferromagnetic (FM) layer is parallel (antiparallel) to its neighbour FM layers, as shown in Figure 1.1. The MR ratio is defined by

$$
M R=\Delta R / R=\left(R_{\mathrm{AP}}-R_{\mathrm{P}}\right) / R_{\mathrm{P}}
$$

where $R_{\mathrm{P}}$ and $R_{\mathrm{AP}}$ represent the resistance of parallel and antiparallel configurations of the FM magnetization, respectively. The GMR effect depends on the spin-dependent scattering of electrons, as shown in Figure 1.2. The electrons with spin aligned with the same direction of the magnetization of FM layer can easily go through all the FM layer leading to a small resistance. However, electrons with spin aligned with the different direction of the magnetization of the magnetic layers will be scattered so that the resistance is high. The main application of GMR is magnetic 


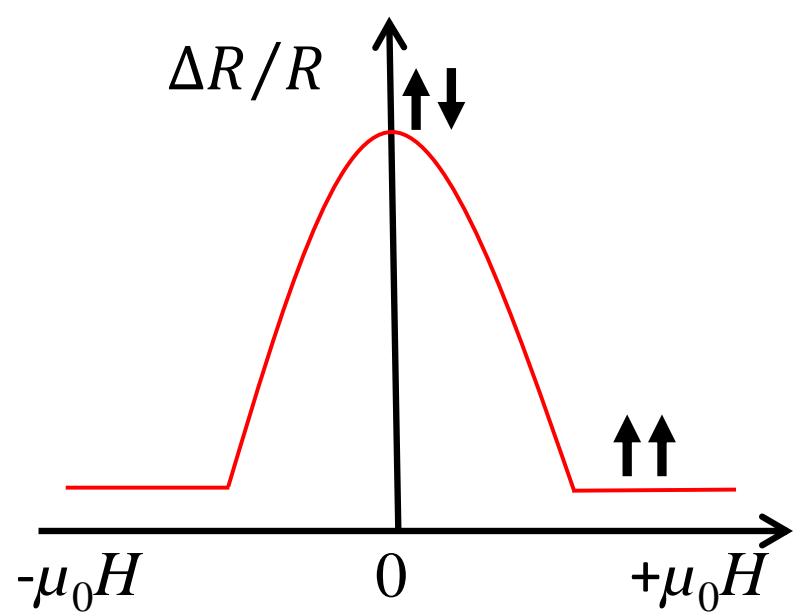

Figure 1.1: Typical GMR curve, the magnetoresistance as a function magnetic field.

field sensors which can be used as read heads in magnetic hard disk drives (HDD).

Another significant progress of spintronics is the discovery of tunneling magnetoresistance (TMR) in the magnetic tunnel junctions (MTJs) with $\mathrm{FM} /$ insulator/FM structure at room temperature for device applications. The TMR effect is similar to GMR, in which the resistance depends on the relative magnetization configuration of two FM layers. The MR signals can be dramatically improved by using insulators, such as $\mathrm{MgO}$, instead of using a metallic spacer. The value of TMR follows Julliere's formula [15]:

$$
T M R=\frac{R_{\mathrm{AP}}-R_{\mathrm{P}}}{R_{\mathrm{AP}}}=\frac{2 P_{1} P_{2}}{1-P_{1} P_{2}},
$$

where $P_{1}$ and $P_{2}$ are the tunneling spin polarizations for the two ferromagnetic layers. Experimentally, giant TMR ratios have been reported by Shinji Yuasa and Stuart Parkin independently in $\mathrm{Fe} / \mathrm{MgO} / \mathrm{Fe}$ and $\mathrm{CoFeB} /$ $\mathrm{MgO} / \mathrm{CoFeB}$ mutilayers, respectively $[16,17]$. By using MTJs as the read heads in ultrahigh-density HDDs with an areal density of $1.402 \mathrm{~Tb} / \mathrm{in}^{2}$ have been realized [18]. These two magnetization configurations can be 
(a) FM1 NM FM2
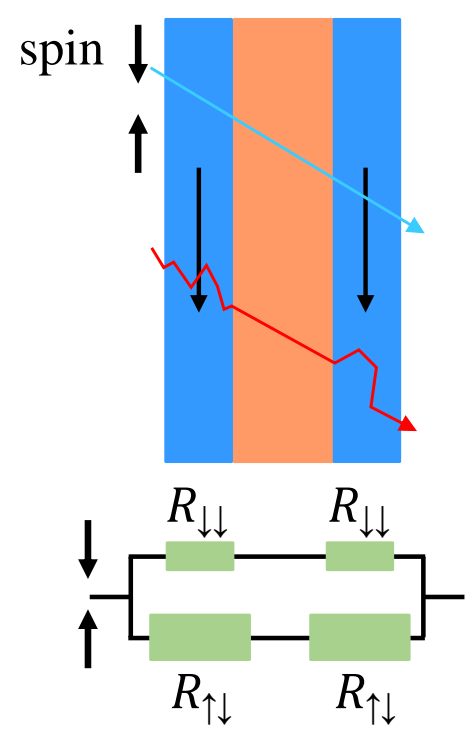

parallel, low $R$ (b) FM1 NM FM2
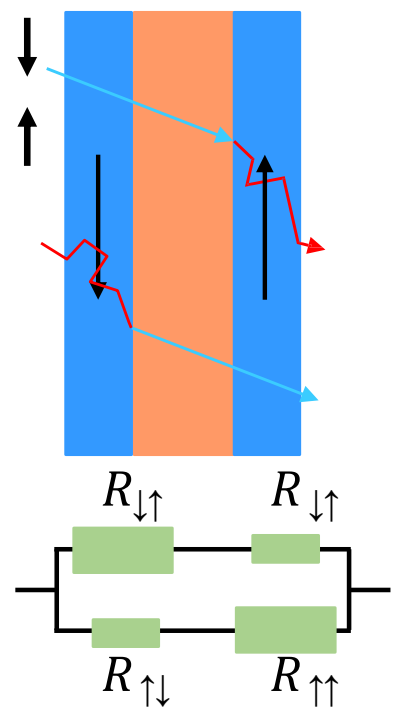

antiparallel, high $R$

Figure 1.2: Schematic of the mechanism of GMR. FM: ferromagnetic layer (arrows indicate the direction of magnetization), NM: non-magnetic layer. Electrons with spin up and down scatter differently in the multilayer. The bottom shows the equivalent circuit diagrams for the total resistances. $R_{\downarrow \downarrow}\left(R_{\uparrow \downarrow}\right)$ denotes the resistance for electrons with spin aligned with the same(different) direction of the magnetization of FM layer.

used for storing information: low resistance state (parallel) correspnds to the logical bit 0 and the other corresponds to 1 . This is the concept of MRAM. The memory cells are MTJs with non-volatile behaviour meaning that they can retain data even after power is removed so that they are energy efficient. The first generation of MRAM is toggle-MRAM in which the magnetization of FM layer is switched by a local magnetic field so that the information can be written. However, the required magnetic field generated by a large current is not possible to change the magnetization of just one cell if the cells are close together which limits its downsize scalability and energy efficiency. 


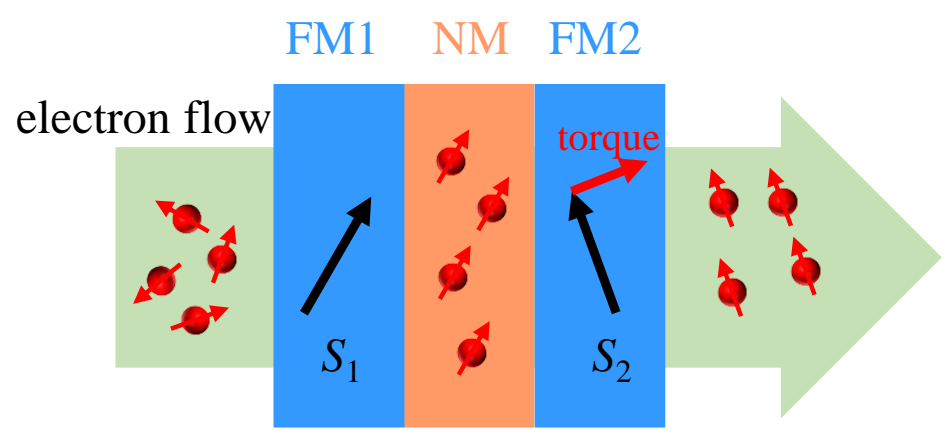

Figure 1.3: Schematic of the mechanism of spin transfer torque.

Spin transfer torque (STT) gives a chance to manipulate the magnetic moment of a FM layer with a spin-polarized current without applying an external magnetic field $[19,20]$. As shown in Figure 1.3, electrons flow from FM1 to FM2 layer. Once electrons pass through the FM1 layer, the spins of electrons exhibit the same direction as that of the magnetization in the FM1 layer, and thus the current becomes spin-polarized. Then, the angular momentum of the spin transfers to the magnetic moment of the FM2 layer as a spin transfer torque which enables to rotate the magnetic moment of FM2 to align with the direction of the magnetic moment of FM1. The concept of spin transfer torque has opened a new direction in spintronics: writing a magnetic configuration with an electrical current, and thus created the second generation of MRAM, called STT-MRAM [21]. Spin-orbit torque (SOT) is another promising method to switch the magnetization of a FM layer, which is known to be more efficient and faster than STT [22]. In principle, for a heavy metal/FM bilayer, a spin-polarized current can be generated from the heavy metal by spin-orbit scattering, and it then diffuses into the FM layer and exerts a SOT which can lead to a switching of the magnetization [23].

One of the alternative memories is the domain wall (DW) racetrack memory originally proposed by Stuart Parkin by using the magnetic domains as information carriers in nanowires [24]. In the racetrack memory, the magnetic domains are used to store information. The informa- 


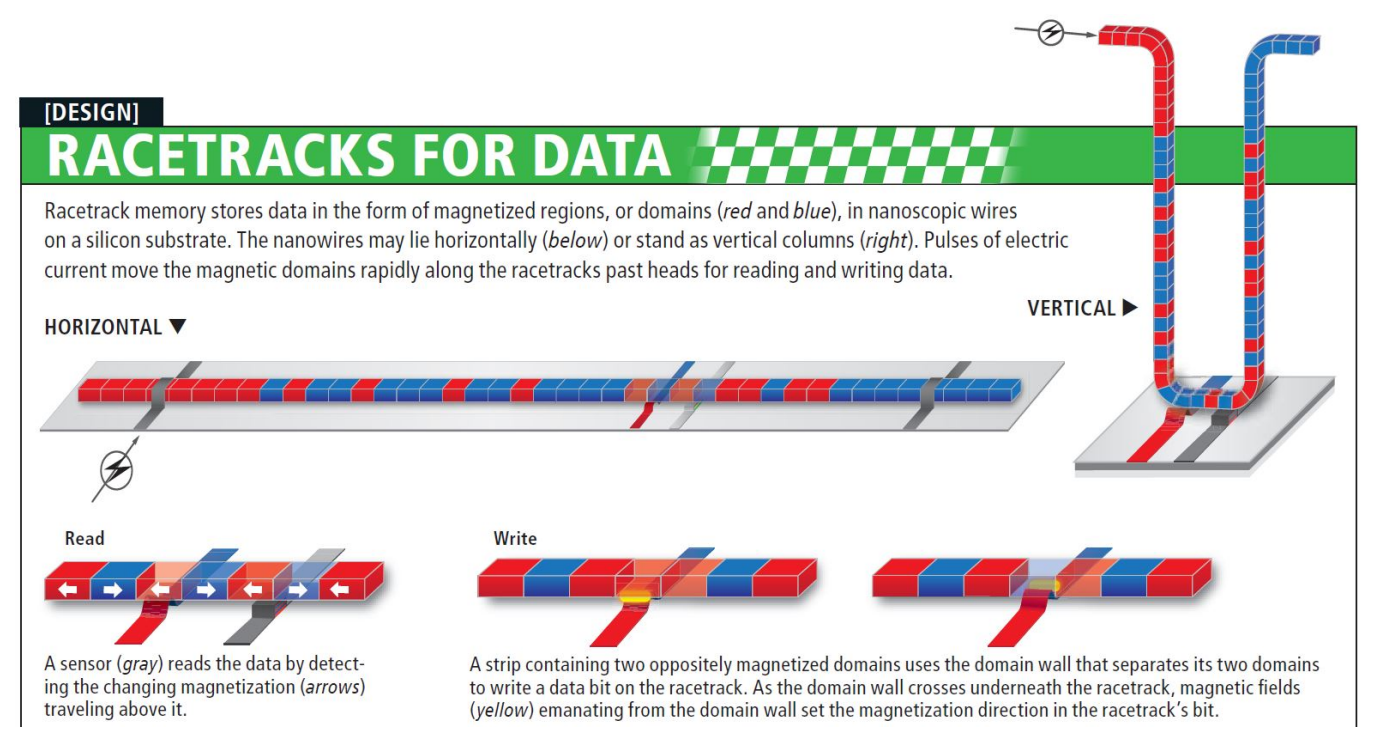

Figure 1.4: Schematic of magnetic DW racetrack memory [30].

tion can be manipulated by moving the DWs by injecting a spin-polarized current pulse, as shown in Figure 1.4. However, the velocity of ordinary DWs is not fast, around $100 \mathrm{~m} / \mathrm{s}$ with a current density of $10^{8} \mathrm{~A} \mathrm{~cm}^{-1}$ [25]. The pinning effect and DW structural instability limit the improvement of velocity. Replacing ordinary domain walls with chiral magnetic structures can help to solve these drawbacks and significantly improve the velocity due to phenomena, like perpendicular magnetic anisotropy, Dzyaloshinskii-Moriya interaction [26, 27], and spin Hall effect [28], derived from spin-orbit coupling [29].

To achieve an ultralow current density, $10^{6} \mathrm{~A} / \mathrm{cm}^{2}$, for racetrack memory, researchers recently started to use magnetic skyrmions as an information carrier [6]. Magnetic skyrmions are solitons with a nanoscale dimension which have chiral spin textures. They can be moved as the behaviour of a particle. Skyrmions have been experimentally observed in bulk materials $[31,32,33]$ and thin films [34, 8, 9]. Among them, ferromagnet/heavy metal ultrathin films exhibit skyrmions at room temperature, which allows them to be used in ultralow power and ultrahigh density devices 
$[6,35]$. Therefore, I am focusing on generating skyrmions in ferromagnet (Heusler alloys)/heavy metal ultrathin film system.

\subsection{Origin of magnetism}

The magnetism of materials originates from the orbital and spin magnetic moment of electrons of atoms. In classical physics, an electron is considered as a charged particle. Phenomenally, the orbital magnetic moment is caused by the electron revolving around the atom nucleus. The spin magnetic moment is induced from the rotation of electron around its own axis. In simple words, since an electron has charge, magnetic moment appears just like a magnetic field is generated when an current passes through a loop of wire, as shown in the Figure 1.5. Now people know electron shows the wave-particle duality. The microscopic theory of magnetism is based on the quantum mechanics of electronic angular momentum. The orbital magnetic moment along the $z$-direction is

$$
m_{z}=-\frac{e}{2 m_{e}} m_{\ell} \hbar, \text { where } m_{\ell}=0, \pm 1, \pm 2, \ldots
$$

Here $m_{e}$ and $m_{\ell}$ is the mass of electron and orbital magnetic quantum number. The spin magnetic moment along the $z$-direction is

$$
m_{z}=-\frac{e}{m_{e}} m_{s} \hbar, \text { with } m_{s}= \pm, \frac{1}{2},
$$

where $m_{s}$ is the spin magnetic quantum number, $\hbar=6.58 \times 10^{-19} \mathrm{eV} \cdot \mathrm{s}$ and $e=1.6 \times 10^{-19} \mathrm{C}$. However, for materials with full orbits, the magnetic moment is zero because the paired electrons with opposite spins cancel their magnetic moment. Thus, only the unpaired electrons contribute a net magnetic moment. For instance, transition metals (like $\mathrm{Co}, \mathrm{Fe}$ and $\mathrm{Ni}$ ) and rare-earth elements (like $\mathrm{Nd}$ and $\mathrm{Sm}$ ) with unpaired electrons in their $d$ or $f$ orbital, respectively, show strong magnetic moments. 

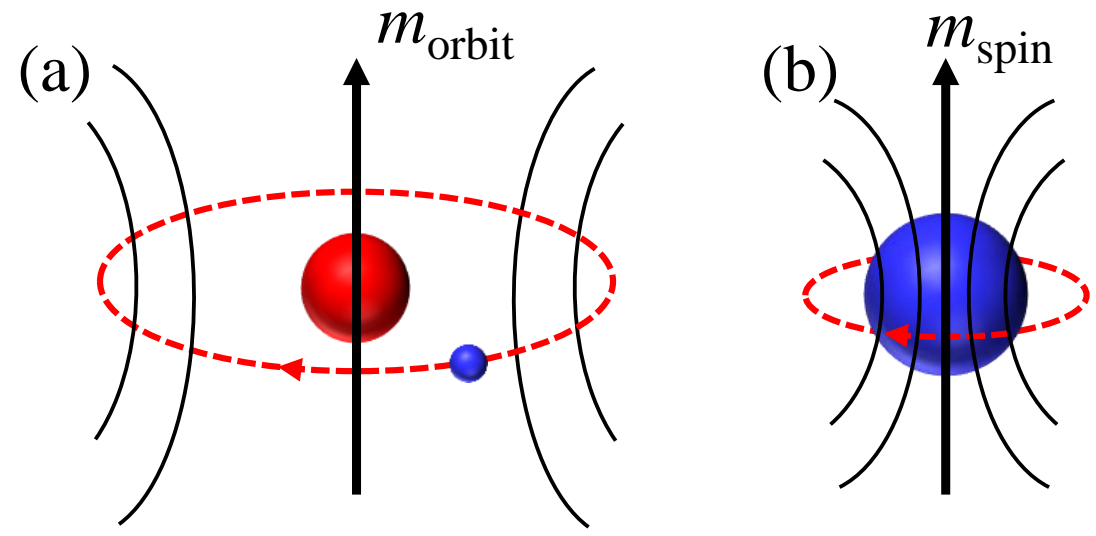

Figure 1.5: Magnetic moment associated with orbital motion (a) and spin (b) of an electron.

For different magnetic materials, their magnetization $(M)$ is influenced by an external magnetic field. $\mathrm{M}$ is defined by the magnetic moment per unit volume $(\mathrm{M}=\mathbf{m} / \Delta V)$. In terms of the magnetic susceptibility, $\chi=\mathbf{M} / \mathbf{H}$, which represents the sensitivity of response to a magnetic field $\mathbf{H}$, the different behaviours of magnetic materials can be categorized diamagnetism, paramagnetism, ferromagnetism, antiferromagnetism, ferrimagnetism and superparamagnetism.

\subsection{Ferromagnetism}

The characteristic feature of ferromagnetism is the spontaneous net magnetization in the absence of magnetic field due to the strongly parallel alignment of magnetic moments, as shown in Figure 1.6(a). This preferential alignment can be ascribed to the exchange interaction between the neighboring spins which is equivalent to a large internal magnetic field. It should be noted that magnetic domains exist without external magnetic field, in which the magnetic moments are in a uniform direction, but the direction of alignment varies from domain to domain in a random behaviour, as shown in Figure 1.6(c). After applying a large enough magnetic 
(a)

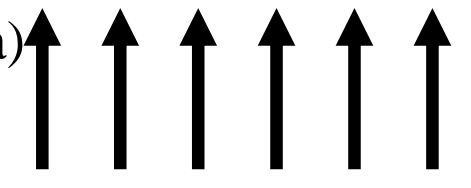

$T<T_{\mathrm{c}}$

(c)

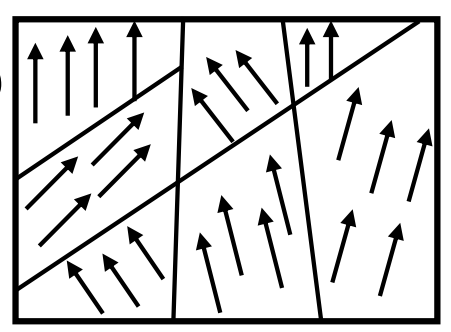

without $\mu_{0} H$

(e)

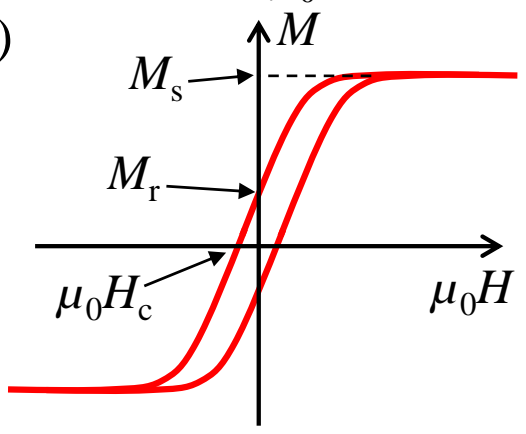

(b)

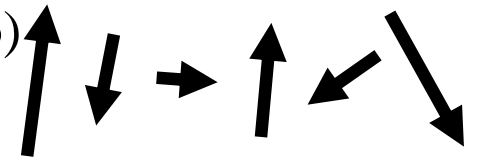

$T>T_{\mathrm{c}}$

(d)

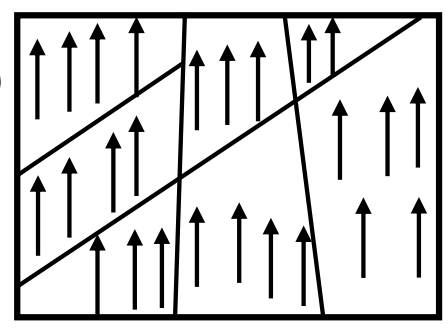

with $\mu_{0} H$

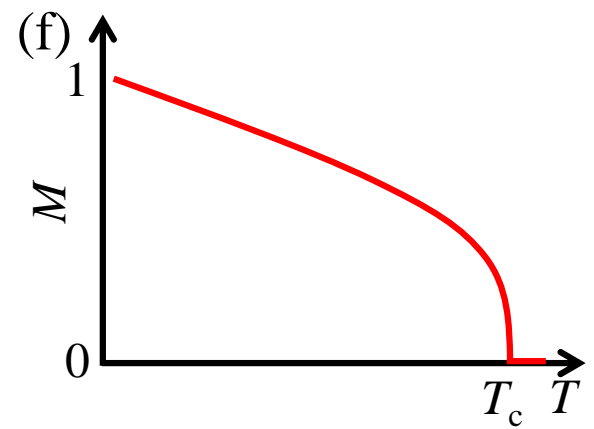

Figure 1.6: (a) Spontaneous magnetization below $T_{\mathrm{c}}$. (b) The random behaviour of magnetization above $T_{\mathrm{c}}$. (c)-(d) The alignment of magnetic domains without or with magnetic field, respectively. (e) Hysteresis loop. (f) The magnetization as a function of temperature.

field, the magnetic moments in all domains will rotate to the direction of magnetic field (see Figure 1.6(d)) and reach to the maximum magnetization called saturation magnetization $\left(M_{\mathrm{s}}\right)$. The plot of the magnetization with magnetic field is called hysteresis loop, as shown in Figure 1.6(e). The magnetization at zero magnetic field is called remanent magnetization $\left(M_{\mathrm{r}}\right)$ meaning that ferromagnets can retain part of or all magnetization once the magnetic field is removed. Another important parameter is the coercive field $\left(\mu_{0} H_{\mathrm{c}}\right)$ which represents the ability of a ferromagnetic mate- 
rial to overcome a magnetic field without becoming magnetically random. The hysteresis loop is a very essential characterization to understand the response of ferromagnetic materials under a magnetic field, which provides the critical parameters, like $M_{\mathrm{s}}, M_{\mathrm{r}}$ and $\mu_{0} H_{\mathrm{c}}$.

The ferromagnetism is also related to the temperature. Even though exchange interaction in ferromagnets is very strong, the thermal energy can eventually overcome it and result in a random order of magnetic moments at a critical temperature called Curie temperature $\left(T_{\mathrm{c}}\right)$. Above the $T_{\mathrm{c}}$, the ferromagnet is magnetically disordered (see Figure 1.6(b)), and the $M_{\mathrm{r}}$ reaches to zero. Figure 1.6(f) shows a typical plot of normalized magnetization $v s$ temperature. The elements $\mathrm{Fe}, \mathrm{Ni}$ and $\mathrm{Co}$ and many of their alloys are typical ferromagnetic materials. Ferromagnetic materials are very important for various industrial applications, especially, spintronics. Here, I am focused on making this type of materials in nanothin films.

\subsection{Magnetic interactions, anisotropies and en- ergy}

In this section, the magnetic interactions, anisotropies and the corresponding energy in ferromagnetic thin films will be discussed, which determine the total energy of magnetic moment and thus give rise to various magnetic configurations.

\subsubsection{Exchange interaction}

The exchange interaction is a consequence of the Pauli exclusion principle, which forbids two electrons to occupy the same quantum state and results in the Coulomb repulsion of two neighbouring electrons. Because electrons cannot be in the same state with the same spin, and different spin configurations of the electrons, like $\uparrow_{i} \uparrow_{j}$ and $\uparrow_{i} \downarrow_{j}(\uparrow(\downarrow)$ corresponds to spin up(down)), give rise to an energy difference between them, the exchange 
interaction between two neighboring atoms depends on the relative orientations of their spins. Heisenberg generalized the exchange interaction Hamiltonian for atomic spins $\mathbf{S}_{i}$ and $\mathbf{S}_{j}$.

$$
H_{\mathrm{ex}}=-2 \mathcal{J} \mathbf{S}_{i} \cdot \mathbf{S}_{j},
$$

where $\mathcal{J}$ is the exchange constant. $\mathcal{J}>0$ represents a ferromagnetic interaction, which tends to align the two spins parallel. The total exchange energy can be written as:

$$
E_{\text {ex }}=A \int_{V}\left[\left(\nabla M_{\mathrm{x}}\right)^{2}+\left(\nabla M_{\mathrm{y}}\right)^{2}+\left(\nabla M_{\mathrm{z}}\right)^{2}\right] d V,
$$

where $M_{\mathrm{xyz}}$ are different components of the magnetization, $V$ is the volume of the magnetic material and $A$ is the exchange stiffness.

\subsubsection{Zeeman energy}

The Zeeman energy is the energy of a magnetic moment under an external magnetic field. It leads to the alignment of the magnetization along the applied magnetic field. It is expressed as

$$
E_{\mathrm{ze}}=-\mu_{0} \int_{V} \mathbf{M} \cdot \mathbf{H} d V,
$$

where $\mathbf{M}$ and $\mathbf{H}$ are the magnetization and magnetic field, respectively.

\subsubsection{Magnetic dipolar anisotropy}

Magnetic dipolar anisotropy or shape anisotropy originates from the long range magnetic dipolar interaction. The source and sink of the magnetization look like the positive and negative magnetic charge which arise at the surface and give rise to the demagnetizing field or stray field, as shown in Figure 1.7. The demagnetizing field is given by: $\mathbf{H}_{\mathrm{d}}=-\left(\mathcal{N}_{\mathrm{x}}+\mathcal{N}_{\mathrm{y}}+\mathcal{N}_{\mathrm{z}}\right) \mathbf{M}$, where $\mathrm{M}$ is the magnetization vector and $\mathcal{N}$ is the shape dependent demagnetizing tensor. 


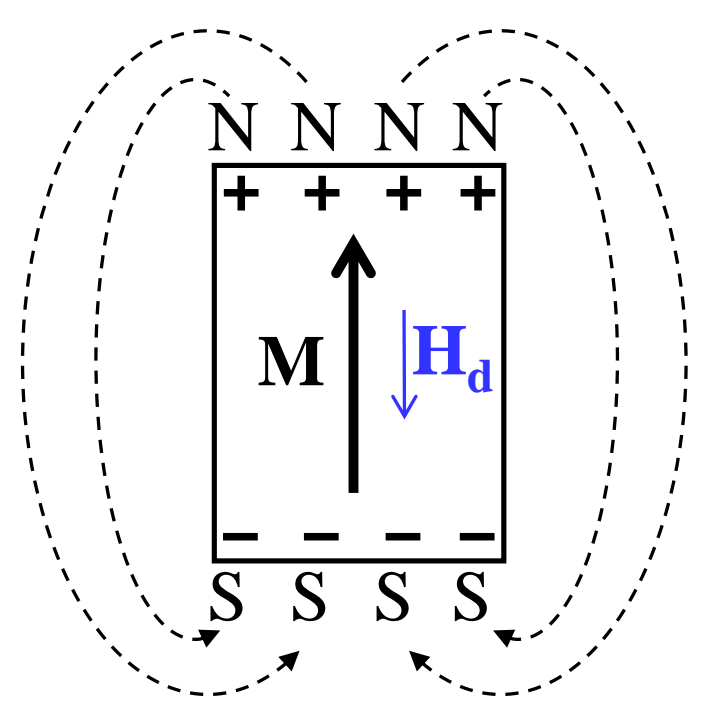

Figure 1.7: Schematic of demagnetizing field.

For a thin film, all tensor elements are zero except $\mathcal{N}_{\mathrm{z}}=1$. The dipolar anisotropy energy can be expressed as

$$
E_{\mathrm{d}}=-\frac{\mu_{0}}{2} \int_{V} \mathbf{M} \cdot \mathbf{H}_{\mathrm{d}} d V
$$

\subsubsection{Magnetocrystalline anisotropy}

Magnetocrystalline anisotropy happens when energy is needed to magnetize the material along a certain crystalline axis. The energy cost is at minimum along easy axes and maximum along hard axes. For materials with a cubic lattice structure, like Fe, the energy is given by

$$
E_{\mathrm{mc}}=K_{\mathrm{c} 0}+K_{\mathrm{c} 1}\left(\alpha_{1}^{2} \alpha_{2}^{2}+\alpha_{2}^{2} \alpha_{3}^{2}+\alpha_{1}^{2} \alpha_{3}^{2}\right)+K_{\mathrm{c} 2}\left(\alpha_{1}^{2} \alpha_{2}^{2} \alpha_{3}^{2}\right)+\ldots
$$

where $\alpha$ gives the cosine of the angle between the magnetization direction and the crystal axis and $K_{\mathrm{c} 0}, K_{\mathrm{c} 1}$ and $K_{\mathrm{c} 2}$ are anisotropy constants. Usually the higher order terms are ignored due to their small contributions. 


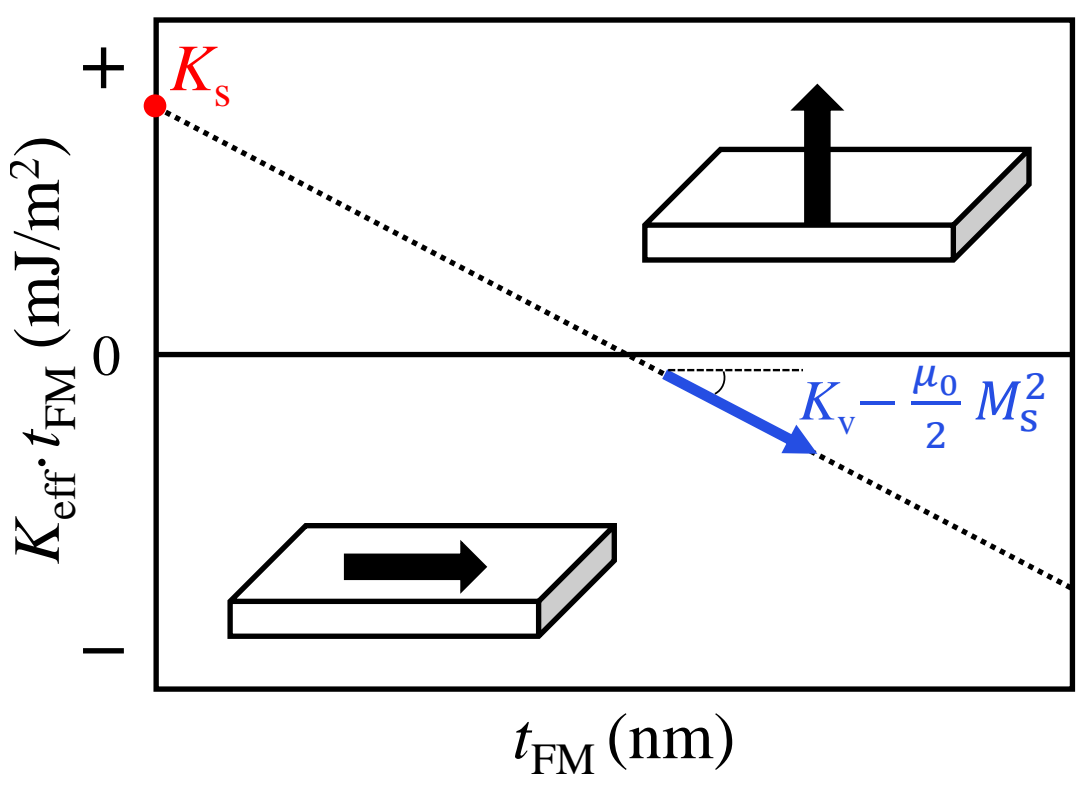

Figure 1.8: Typical curve about $K_{\text {eff }} \cdot t$ versus the thickness of ferromagnetic layer in multilayers. The flat plates and arrows denote the thin film and the direction of magnetization, respectively.

\subsubsection{Perpendicular magnetic anisotropy}

For a thin magnetic film placed between two interfaces, the total anisotropy is usually expressed as an effective anisotropy per unit volume

$$
K_{\text {eff }}=\left(K_{\mathrm{v}}-\frac{\mu_{0}}{2} M_{\mathrm{s}}^{2}\right)+\frac{K_{\mathrm{s}}}{t},
$$

where $K_{\mathrm{v}}$ accounts for bulk anisotropy contribution, $(1 / 2) \mu_{0} M_{\mathrm{s}}^{2}$ represents the demagnetizing energy, $t$ is the thickness of the ferromagnetic layer and $K_{\mathrm{s}}$ is the interfacial anisotropy originating from the two interfaces. Experimentally, the product $K_{\text {eff }} \cdot t$ can be plotted against $t$ to determine the various contributions. The slope of this curve gives the net bulk anisotropy, whereas the intercept with the vertical axis yields the net interfacial anisotropy, see Figure 1.8. For $K_{\mathrm{s}}>0$, thin films show PMA, favoring a magnetization perpendicular to the interface. This anisotropy was firstly observed in Co/X (X = Pd, Pt, Ir, Au) mutilayers [36, 37, 38]. 
The presence of nonmagnetic heavy metals at such interfaces modified the interfacial orbital angular momentum of Co due to spin-orbit interactions [39]. Later on, perpendicular anisotropy was also found in thin films with interfaces between the ferromagnet and nonmagnetic oxides such as $\mathrm{MgO}$ and $\mathrm{AlO}_{x}$, attributed to the nature of the bonding between the metal and oxygen ions at the interface [40,41, 42].

\subsubsection{Dzyaloshinskii-Moriya interaction}

DMI is an antisymmetric exchange interaction, which was first proposed by Dzyaloshinskii in 1957 to explain weak ferromagnetism due to canted magnetic moments in antiferromagnets [26]. Later, Moriya linked the existence of this interaction to the spin-orbit coupling mechanism [27].

For ferromagnet/heavy metal ultrathin film bilayers or multilayers with PMA, the DMI can be induced at the interface between the ferromagnetic layer and the heavy metal layer due to the broken inversion symmetry and strong spin-orbit coupling. The Hamiltonian of DMI between two neighbouring spins $\mathbf{S}_{i}$ and $\mathbf{S}_{j}$ can be expressed as

$$
H_{\mathrm{DMI}}=-\mathbf{D}_{i j} \cdot\left(\mathbf{S}_{i} \times \mathbf{S}_{j}\right),
$$

where $\mathbf{D}_{i j}$ is the DMI vector. Therefore, spin $\mathbf{S}_{i}$ can be tilted with respect to $\mathbf{S}_{j}$ by a rotation around the DMI vector $\mathbf{D}_{i j}$, as shown in Figure 1.9. Compared with the exchange interaction favouring collinear spin alignment, DMI induces a non-collinear spin texture with a chirality. A chiral object cannot be superimposed onto its mirror image. The DMI energy can be written as follows

$$
E_{\mathrm{DMI}}=D\left[M_{\mathrm{z}} \frac{\partial M_{\mathrm{x}}}{\partial x}-M_{\mathrm{x}} \frac{\partial M_{\mathrm{z}}}{\partial x}\right]+D\left[M_{\mathrm{z}} \frac{\partial M_{\mathrm{y}}}{\partial y}-M_{\mathrm{y}} \frac{\partial M_{\mathrm{z}}}{\partial y}\right],
$$

where $D$ is the DMI coefficient. 


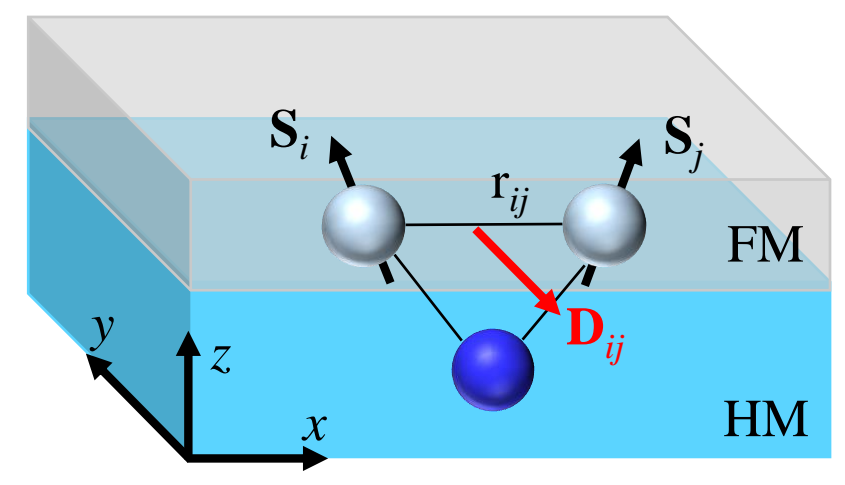

Figure 1.9: Schematic of DMI between two neighbouring spins $\mathbf{S}_{i}$ and $\mathbf{S}_{j}$ at the interface between ferromagnet and heavy metal layer. The DMI vector $\mathbf{D}_{i j}$ is along the $y$ axis direction.

\subsection{Magnetic spin textures}

This section addresses the nature of magnetic domains and domain walls in thin films. Chiral domain walls and spin texture induced by the DMI are introduced as well.

\subsubsection{Magnetic domains}

A magnetic domain is a region in which the magnetic moments are spontaneously aligned parallel due to the exchange interaction, as shown in Figure 1.10(a). However, for a big size of single domain, surface charges will form and lead to a large demagnetizing field which contributes to the magnetostatic energy and is no longer energetically favorable. In order to minimize this magnetostatic energy, the single domain breaks up into several domains so that the positive and negative magnetic charges close together and produce a pole-free configuration. For instance, the magnetostatic energy can be significantly decreased if the single domain splits into two domains magnetized in opposite directions, as shown in Figure 1.10(b). The equilibrium domain configuration depends on the result of minimizing the total free energy. For thin films, there are five main 

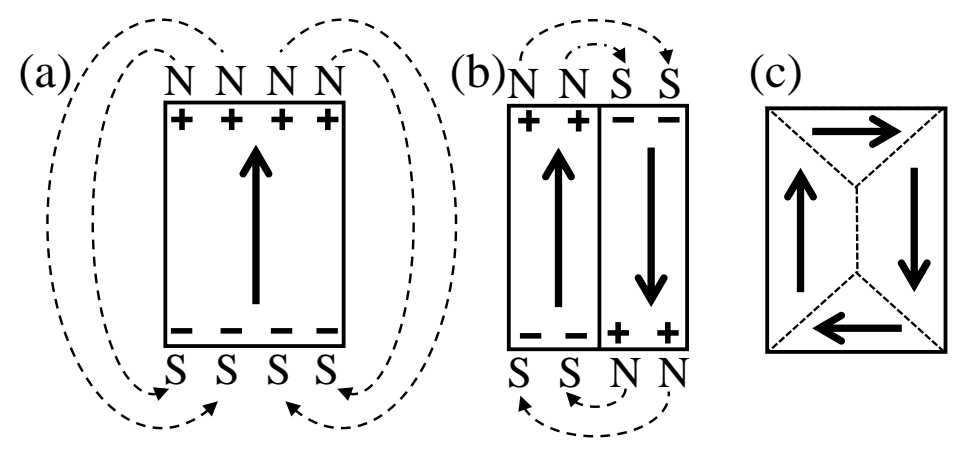

Figure 1.10: (a) A single domain, (b) A double-domain and (c) a multi-domain. The arrow and dash lines represent magnetic moment and demagnetizing field, respectively.

terms, which have been discussed in Section 1.5, have to be considered:

$$
E_{\mathrm{tot}}=E_{\mathrm{ex}}+E_{\mathrm{ze}}+E_{\mathrm{d}}+E_{\mathrm{mc}}+E_{\mathrm{DMI}}
$$

Eventually, the domains will reach into an equilibrium configuration with a specific size, for instance, like the texture in Figure 1.10(c).

\subsubsection{Domain walls}

Between two neighbouring domains, there is a transition region called domain wall (DW) in which the magnetic moment gradually rotates from one direction to another. The DW width is determined by the competition between the exchange interaction and effective magnetic anisotropy and is given by

$$
\Delta=\sqrt{\frac{A_{\mathrm{ex}}}{K_{\mathrm{eff}}}}
$$

where $A_{\text {ex }}$ is the exchange stiffness. The DW width is of order 10 to $100 \mathrm{~nm}$ [43]. From this equation, one can know that the exchange interaction tends to keep adjacent magnetic moments parallel and widen the DW, but the effective magnetic anisotropy wants them aligned with a certain direction and narrows the DW. 

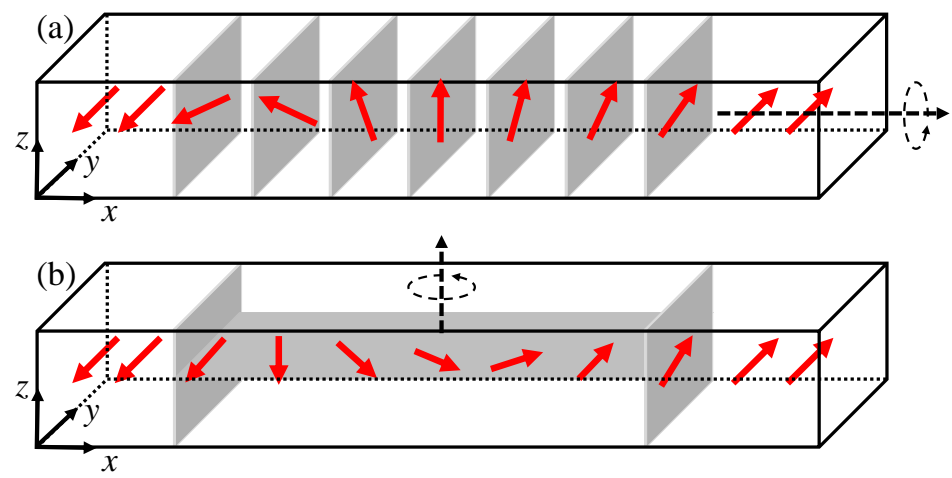

Figure 1.11: Schematic view of magnetic moments rotation in a Bloch wall (a) and Néel wall (b) between two domains with a opposite magnetized direction. The dashed arrows show the axis of rotation of magnetization. The thickness direction is along the $z$ axis.

For thin films, there are two typical types of $180^{\circ}$ DWs: Bloch and Néel walls. In a Bloch wall, the magnetic moments rotate in the $y$ - $z$ plane perpendicular to the surface of films (Figure 1.11(a)). In a Néel wall, the magnetic moments rotate in the $x-y$ plane (Figure 1.11(b)). The Néel wall normally has a higher energy than the Bloch wall because of a higher demagnetizing field created by the nonzero divergence of magnetization. However, when the thickness of the thin film is thinner than the DW width, Néel walls are more stable. There is a simple model to explain it. The wall is approximated by an elliptical cylinder of width $\Delta$ and thickness $t$. For the Bloch wall the demagnetizing factor of this cylinder along the out-of-plane direction is $N_{\text {Bloch }}=\Delta /(\Delta+t)$. If $t>>\Delta$, then $N \approx 0$ suggesting that the demagnetizing field closes to 0 . However, for the Néel wall $N_{\text {Néel }}=t /(\Delta+t)$, the $N_{\text {Néel }}$ will be smaller than $N_{\text {Bloch }}$ once $t$ is thinner than $\Delta$ indicating that the Néel wall has a lower demagnetizing field, as shown in Figure 1.12. One of the great potential application of the DWs is the magnetic DW racetrack memory which has been mentioned in section 1.2 . 


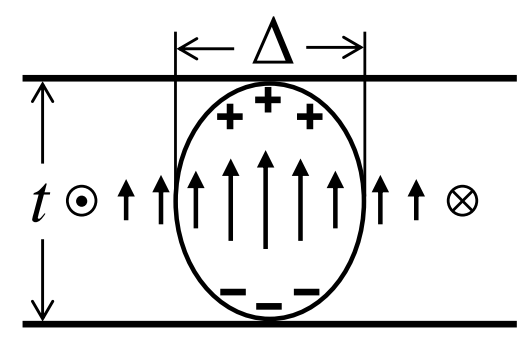

Bloch wall

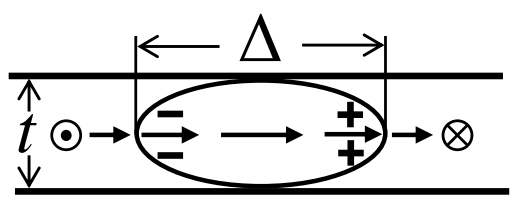

Néel wall

Figure 1.12: Cross section of a Bloch and Néel wall in thin films with different thickness $t$ and wall width $\Delta$. The arrows represent the projection on the cross section plane.

\subsubsection{Chiral domain walls}

Chiral DWs can be found in ferromagnet/heavy metal ultrathin films with PMA due to the competition of DMI favouring non-collinear spin texture, exchange interaction, and magnetic anisotropy. The chirality of the DW is determined by the sign of the $D$. For a positive DMI $(D>0)$, the spins rotate anticlockwise (ACW) from up to down and back to the up state creating a so-called left-handed Néel wall, as shown in the Figure 1.13(top). On the other hand, spins rotating clockwise (CW) from up to down and back to the up state is a so-called right-handed Néel wall with a negative DMI, as shown in the Figure 1.13(bottom). For the chiral DWs, the current-induced DW velocity can reach to $350-400 \mathrm{~m} / \mathrm{s}$ [44, 45]. Instead of using chiral DWs, skyrmions are another option to improve the scaling, reliability, and reproducibility of racetrack memory [6].

\subsubsection{Skyrmions}

A magnetic skyrmion is a chiral spin texture induced by DMI [46]. There are two typical types of magnetic skyrmions: Néel-type and Bloch-type skyrmions defined by the directions of the rotation. Figure 1.14(a) shows skyrmions with the spin rotating from the $\downarrow$ direction at centre to the $\uparrow$ 


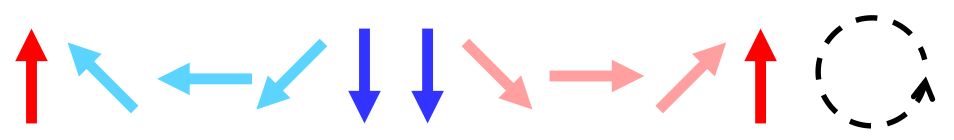 \\ left-handed Néel wall (ACW)

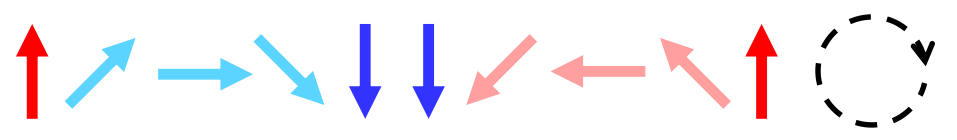 \\ right-handed Néel wall (CW)}

Figure 1.13: The spin rotation of chiral Néel domain walls.

direction at the edge. The topological spin texture of skyrmions can be defined by the topological charge or skyrmion number $S$, which characterizes the winding of the normalized local magnetization, M. In the twodimensional limit, the topological number is:

$$
S=\frac{1}{4 \pi} \int \mathbf{M} \cdot\left(\partial_{x} \mathbf{M} \times \partial_{y} \mathbf{M}\right) d x d y .
$$

To understand the chirality of skyrmions, a simple one-dimensional example is given to visualise the skyrmion number. The spin textures along the cross-section through the center for the Néel-type skyrmion have been shown in Figure 1.14(b), which is the same as the chiral Néel DW (see Figure 1.13). The spin $\uparrow$ rotates $360^{\circ}$ and back to $\uparrow$ direction with the chiral symmetry breaking so that the $S=1$. However, for the topologically trivial DWs, as shown in Figure 1.14(c), the net rotated angle is zero meaning that $S=0$. For a single Néel or Bloch skyrmion, the value of $S$ is quantized and given by \pm 1 . The sign of $S$ represents the direction of the spin at the center, i.e. $S=1(S=-1)$ corresponds to spin $\downarrow(\uparrow)$. This non-trivial topology determines the properties of skyrmions. Firstly, the skyrmion is topologically protected because there is a topological barrier to prevent it collapsing into other spin configuration with different $S$. What's more, skyrmion shows a solitonic nature: moving or interacting as a particle. Because of these unique properties, it shows great potential applications for magnetic memory, logic and computing applications [7, 35]. 
(a)

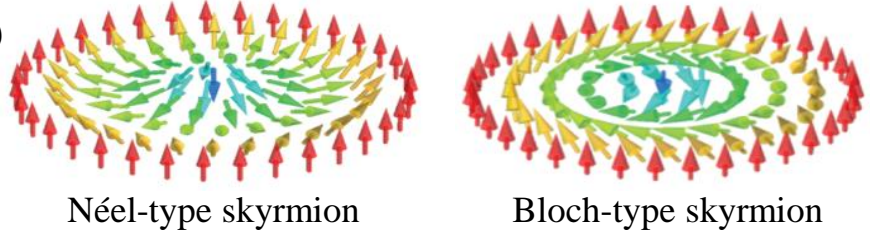

(b)
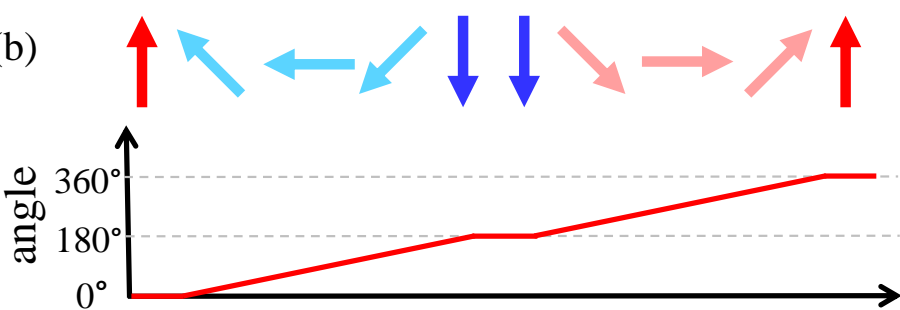

Topologically non-trivial domain wall

(c)
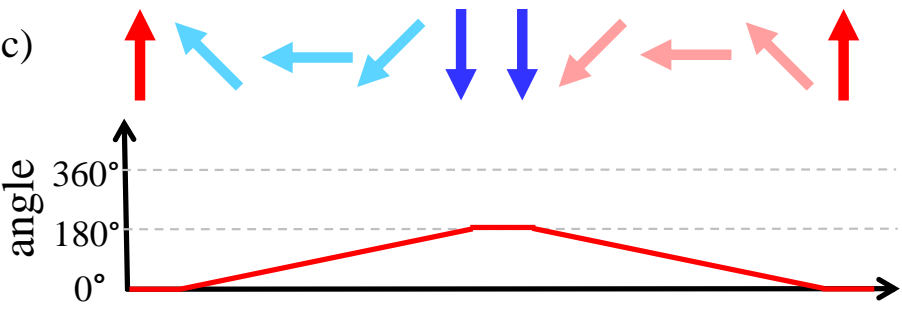

Trivial domain wall

Figure 1.14: (a) Spin texture of skyrmions. (b) The spin profile from the crosssection of Néel-type skyrmion and its angular progression which is the same as the non-trivial DWs. (c) The angular progression of the trivial DWs.

Skyrmions have been experimentally observed in both bulk materials with a non-centrosymmetric lattice $[31,32,33]$ and thin film magnetic multilayers with DMI at the interfaces [34, 8, 9]. Among them, ferromagnet/heavy metal ultrathin films exhibit Néel-type skyrmions at room temperature which allows them to be used in ultralow power and ultrahigh density devices $[6,35]$. Typical skyrmion sizes reported in the literature range between 4 and $4200 \mathrm{~nm}$ in various multilayer thin film systems [47].

Since the skyrmion is a quasiparticle with nanoscale dimension, it can be used as an information carrier and becomes an alternative option for magnetic racetrack memory (see Figure 1.15). Skyrmions can be moved 


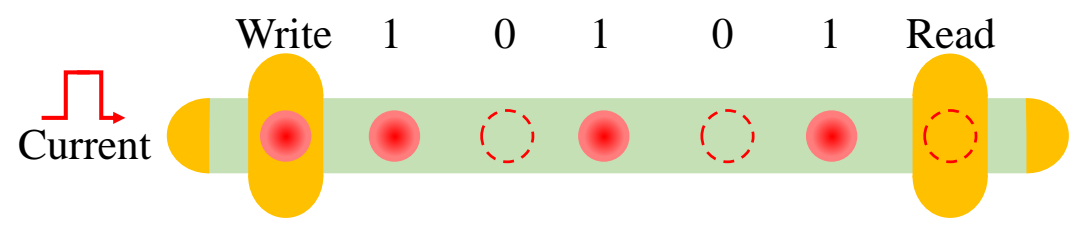

Figure 1.15: Skyrmion-based magnetic racetrack memory. The presence of a skyrmion code data bit 1 and the absence of a skyrmion code data bit 0 . Skyrmion driven by a current pulse. The red dot denotes skyrmion.

under ultralow current densities, $10^{2} \mathrm{~A} \mathrm{~cm}^{-1}$ [48, 49], five orders of magnitude below the critical current density above which chiral DWs can be moved with spin-orbit torques. However, the the velocity is very slow $10^{-4} \mathrm{~m} / \mathrm{s}$. Recently, skyrmions have been driven by a current density of $5 \times 10^{7} \mathrm{~A} \mathrm{~cm}^{-1}$ at a relatively high speed of $100 \mathrm{~m} / \mathrm{s}$ [50]. Besides information memory, skyrmions can be used to build logic, computing, and neuromorphic devices [35].

\subsection{Heusler alloys}

The Heusler compounds were first discovered by Friedrich Heusler in 1903 when he reported that $\mathrm{Cu}_{2} \mathrm{MnAl}$ surprisingly exhibits a ferromagnetic behaviour at room temperature [51], although none of its constituent elements shows magnetism. Until 1934, Otto Heusler [52] and Albert Bradley [53] found that this ferromagnetic property strongly depends on the crystal structure. Today, there are more than 1000 Heusler compounds with a wide range of material properties from semiconductors, metals to topological insulators with various applications, such as spintronics, thermoelectrics, opto-electronics and so on [54]. Especially for spintronics, due to their high spin polarization, high Curie temperature, low Gilbert damping and tunable material properties, they are ideal materials for spintronics applications, like MRAM, TMR sensor and spin injection [55]. 


\begin{tabular}{|c|c|c|c|c|c|c|c|c|c|c|c|c|c|c|c|c|c|}
\hline H & & & & & & & & & & & & \multirow[b]{2}{*}{ B } & \multirow[b]{2}{*}{ C } & \multirow[b]{2}{*}{$\mathbf{N}$} & \multirow[b]{2}{*}{ O } & \multirow[b]{2}{*}{$\mathbf{F}$} & \multirow{2}{*}{$\begin{array}{c}\mathrm{He} \\
\mathrm{Ne}\end{array}$} \\
\hline $\mathbf{L i}$ & Be & & & & & & & & & & & & & & & & \\
\hline $\mathrm{Na}$ & Mg & & & & & & & & & & & Al & $\mathbf{S i}$ & $\mathbf{P}$ & $\mathbf{S}$ & Cl & $\mathbf{A r}$ \\
\hline $\mathbf{K}$ & $\mathbf{C a}$ & Sc & $\mathbf{T i}$ & V & $\mathrm{Cr}$ & Mn & $\mathrm{Fe}$ & Co & $\mathbf{N i}$ & $\mathbf{C u}$ & $\mathbf{Z n}$ & Ga & Ge & As & Se & $\mathrm{Br}$ & $\mathbf{K r}$ \\
\hline $\mathbf{R b}$ & $\mathbf{S r}$ & $\mathbf{Y}$ & $\mathbf{Z r}$ & Nb & Mo & Tc & $\mathbf{R u}$ & $\mathbf{R h}$ & $\mathbf{P d}$ & $\mathbf{A g}$ & $\mathbf{C d}$ & In & Sn & Sb & $\mathrm{Te}$ & I & $\mathbf{X e}$ \\
\hline Cs & Ba & & Hf & Ta & W & $\mathbf{R e}$ & Os & $\mathbf{I r}$ & $\mathbf{P t}$ & Au & $\mathrm{Hg}$ & Tl & $\mathbf{P b}$ & $\mathbf{B i}$ & Po & At & $\mathbf{R n}$ \\
\hline $\mathbf{F r}$ & $\mathbf{R a}$ & & & & & & & & & & & & & & & & \\
\hline & & & La & Ce & $\operatorname{Pr}$ & $\mathbf{N d}$ & Pm & Sm & Eu & Gd & Tb & Dy & Ho & Er & Tm & $\mathbf{Y b}$ & Lu \\
\hline & & & Ac & Th & $\mathbf{P a}$ & $\mathbf{U}$ & Np & $\mathbf{P u}$ & Am & $\mathbf{C m}$ & Bk & Cf & Es & $\mathbf{F m}$ & Md & No & $\mathbf{L r}$ \\
\hline
\end{tabular}

Figure 1.16: Periodic table of the elements. The Heusler compounds can be formed by combination of the different elements for $X$ (red), $Y$ (blue) and $Z$ (purple) [54].

\subsubsection{Crystal structure}

Ternary inter-metallic Heusler compounds are categorized into two groups $X Y Z$ (half Heusler) and $X_{2} Y Z$ (full Heusler) due to their crystal structures, where $X$ and $Y$ are transition metal elements and $Z$ is a main group element (Figure 1.16). In some cases $Y$ can be replaced by a rare earth or alkaline metal. For half Heusler and full Heusler alloys without disorder, they show $C 1_{b}$ structure and $L 2_{1}$ structure, respectively, as shown in the Figure 1.17(a) and (b). In the unit cell of the $L 2_{1}$ structure, $X$ is at $8 \mathrm{c}\left(\frac{1}{4} \frac{1}{4} \frac{1}{4}\right)$, $Y$ at $4 \mathrm{a}(000)$ and $Z$ at $4 \mathrm{~b}\left(\frac{1}{2} \frac{1}{2} \frac{1}{2}\right)$ in Wyckoff coordinates. Compared with the $L 2_{1}$ structure, the $C 1_{b}$ structure is formed by removing one of the $X$ sites. However, various degrees of disorder give rise to different types of structure. For the B2 structure, the (000) and $\left(\frac{1}{2} \frac{1}{2} \frac{1}{2}\right)$ sites are randomly occupied by $Y$ and $Z$ atoms (Figure 1.17(c)). In addition, a completely random distribution of all three atoms on all sites leads to the $A 2$ structure (Figure 1.17(d)).

The properties of Heusler alloys are strongly related to the order and distribution of the atoms, thus it is very important to determine the crystal structure of Heusler alloy thin films. X-ray diffraction is a simple method 

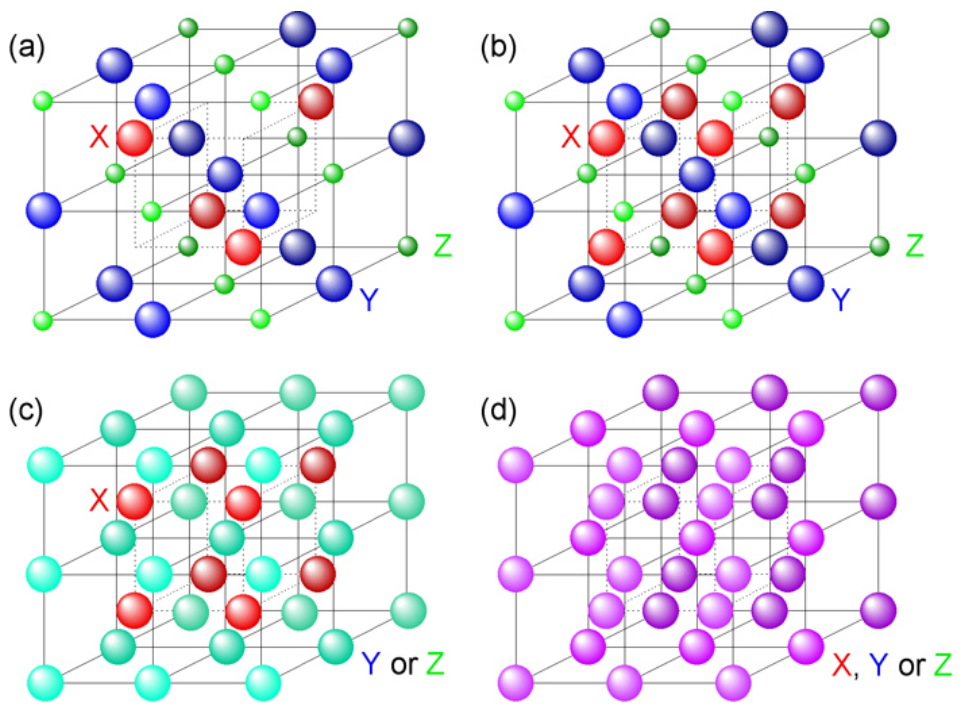

Figure 1.17: Crystal structures. (a) half Heusler alloys $C 1_{b}$ structure, (b) full Heusler alloys $L 2{ }_{1}$ structure, (c) disordered $B 2$ and (d) $A 2$ structure. [56]

to detect the crystal structure. For example, the $L 2_{1}$ structure can be confirmed if there is the existence of both (220) and (111) peaks resulting from the superlattice reflections in the $L 2_{1}$ structure [57].

\subsubsection{Half-metal and magnetism}

The materials with $100 \%$ spin polarization are called half-metals [58]. The spin polarization is defined as the normalized imbalance of the density of states (DOS) of the majority band (spin up, $\uparrow$ ) and minority band (spin down, $\downarrow$ ) at the Fermi level

$$
P\left(E_{\mathrm{F}}\right)=\frac{D_{\uparrow}\left(E_{\mathrm{F}}\right)-D_{\downarrow}\left(E_{\mathrm{F}}\right)}{D_{\uparrow}\left(E_{\mathrm{F}}\right)+D_{\downarrow}\left(E_{\mathrm{F}}\right)},
$$

where $D_{\uparrow}$ and $D_{\downarrow}$ are the DOS of spin-up and spin-down electrons at the Fermi level, respectively. For half-metals, they have a band gap at the minority band like a semiconductor, while the majority band has no gap and exhibits metallic behaviour at the Fermi level, as shown in Figure 1.18(b). 


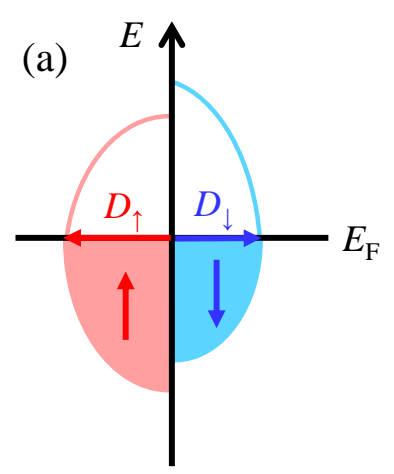

Ferromagnet

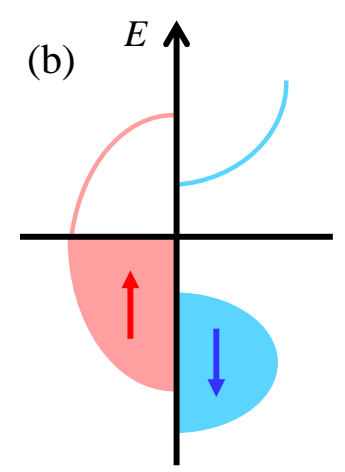

Half-metallic ferromagnet

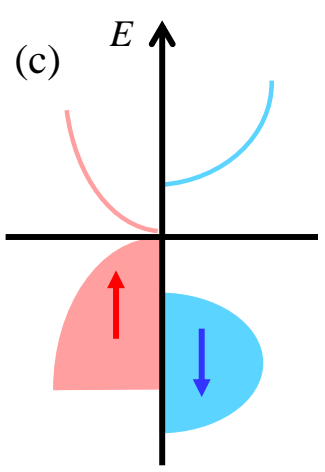

Spin gapless ferromagnet

Figure 1.18: Schematic illustration of density of states for ordinary ferromagnet (a), half-metallic ferromagnet (b) and spin gapless ferromagnet (c). Arrows indicate the majority $(\uparrow)$ and minority $(\downarrow)$ states.

Due to the DOS of the minority band being zero at the Fermi level, this material will be $100 \%$ spin polarized at the Fermi level which makes it ideal for MRAM. For magnetic tunnel junctions, unit cells of MRAM, with ferromagnet/insulator tunnel barrier/ferromagnet structure, the value of TMR is defined by the equation 1.2. In order to obtain a large TMR value, a high spin polarization is necessary. If a material exhibits that one of the DOS equals to zero, one can obtain a $100 \%$ spin polarization which results in a theoretically infinite value of TMR. For typical ferromagnetic materials, like $\mathrm{Fe}, \mathrm{Co}$ and $\mathrm{Ni}$, the spin polarization is below 0.45 [59] due to a nonvanishing DOS of spin-up and spin-down electrons (see Figure 1.18(a)). Experimentally, a large TMR $354 \%$ at $290 \mathrm{~K}$ has been achieved in $\mathrm{Co}_{2} \mathrm{MnSi}$ (halfmetal) $/ \mathrm{MgO} / \mathrm{Co}_{2} \mathrm{MnSi}$ MTJs [60]. What's more, half-metals can be used in spin-injection, spin-filter, and GMR devices [61].

The half Heusler alloy NiMnSb is the first half-metal, predicted by R. A. de Groot in 1983 [63]. The second family of half-metals, $\mathrm{Co}_{2}$-based Heusler compounds, such as $\mathrm{Co}_{2} \mathrm{MnSi}, \mathrm{Co}_{2} \mathrm{MnSn}$ and $\mathrm{Co}_{2} \mathrm{MnAl}[64,65]$, have attracted most of the attention due to their high Curie temperatures (above $600 \mathrm{~K})$. It is well known that half-metallic Heusler alloys follow the Slater- 


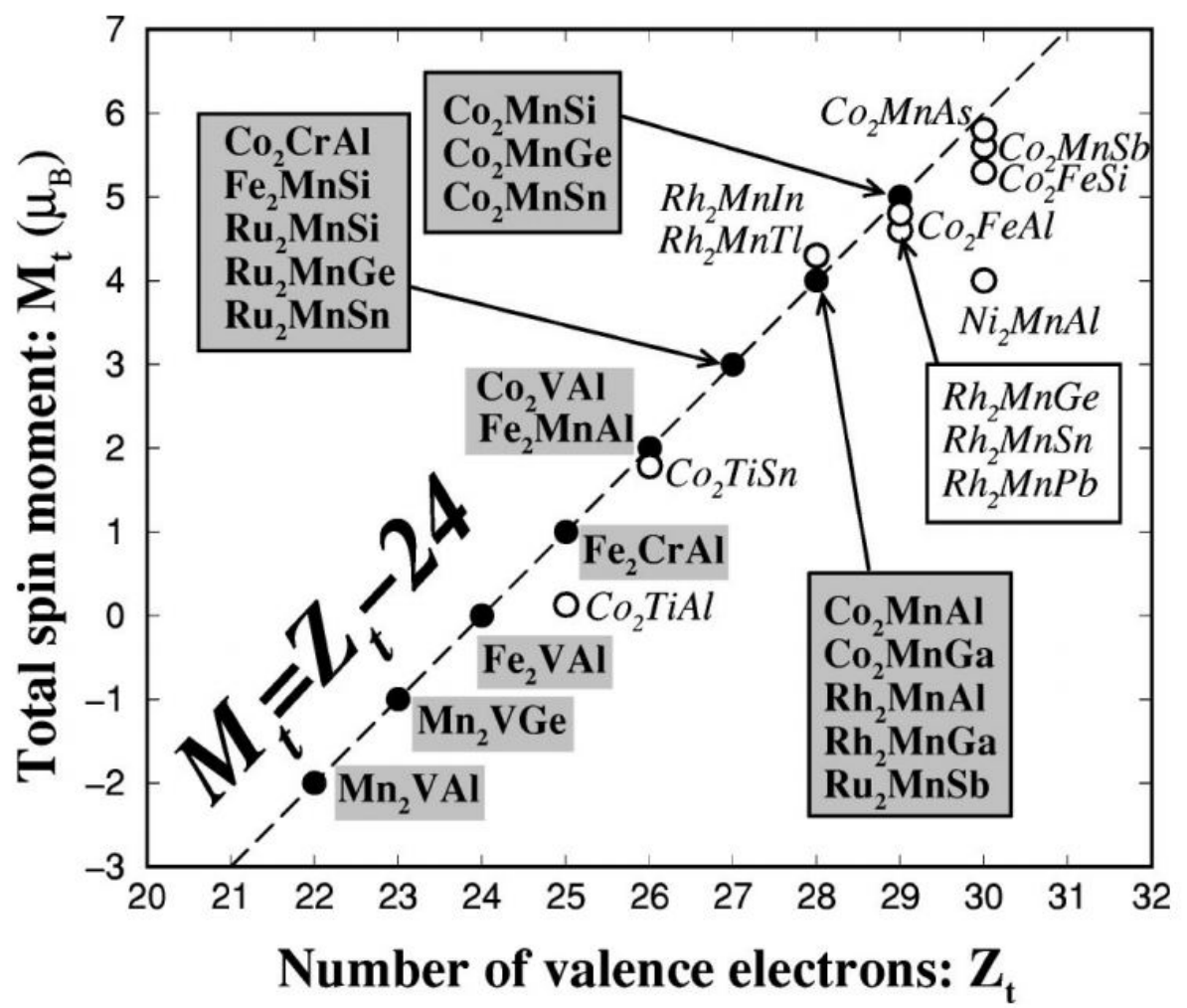

Figure 1.19: Calculated total spin magnetic moments for Heusler alloys. The dashed line represents the Slater-Pauling behaviour [62].

Pauling rule for predicting their total magnetic moment [62]. The electronic structure of these alloys exhibits a gap in the minority density of states at the Fermi level, in which the Fermi energy level is pinned. Therefore, the minority spin density is predefined to a finite value, and hence, the number of majority electrons increases proportional to the total number of electrons. As a consequence, the magnetic moment scales linearly with the number of valence electrons. The total magnetic moment $m$ is given by:

$$
m=N_{\text {maj }}-N_{\text {min }}
$$

where $N_{\text {maj }}$ and $N_{\text {min }}$ are the number of majority states and minority states, respectively. The total number of valence electrons in one unit cell given 
by:

$$
N_{\mathrm{V}}=N_{\text {maj }}+N_{\text {min }}
$$

Thus, one can obtain $m=N_{\mathrm{V}}-2 N_{\min }$. For the $\mathrm{Co}_{2} \mathrm{MnZ}$ half-metals, there are eight $d$, one $s$ and three $p$ band electrons with $N_{\min }=2$ per unit cell. Thus, $m=N_{\mathrm{V}}-24$ (see Figure 1.19 [62]).

\subsubsection{Spin gapless semiconductors, $\mathrm{Mn}_{2} \mathrm{CoAl}$}

Recent studies have reported an interesting class of semiconductor materials that bridge the gap between semiconductors and half-metallic ferromagnets. These materials, called spin gapless semiconductors (SGS), exhibit a band gap in one of the spin channels and a zero band gap in the other (see Figure 1.18(c)) and thus allow for tunable magnetic properties. The first spin gapless magnetic semiconductor, $\mathrm{Mn}_{2} \mathrm{CoAl}$, was experimentally verified by Claudia Felser's group in 2013 [66]. This bulk material shows a magnetic moment of $2 \mu_{B}$ and a Curie temperature of $720 \mathrm{~K}$. In order to develop this new material for spintronics application, researchers start to extend the study of magnetization and disorder for $\mathrm{Mn}_{2} \mathrm{CoAl}$ from bulk to thin films $[67,68]$. Also, perpendicular magnetic anisotropy has been observed in $\mathrm{MgO} / \mathrm{Mn}_{2} \mathrm{CoAl} / \mathrm{Pd}$ thin films $[69,70]$.

\subsubsection{Magnetic Weyl semimetals, $\mathrm{Co}_{2} \mathrm{MnGa}$}

In Weyl semimetals (WSMs), a type of topological semimetal, the valence and conduction bands touch each other at isolated points called Weyl nodes, as shown in Figure 1.20(a). Chiral Weyl fermions, as low-energy quasiparticle excitations around these Weyl nodal points, can be pumped along surface Fermi arcs that connect the paired Weyl nodes. These Weyl nodes can be understood as the monopoles and anti-monopoles of Berry curvature in momentum space [71]. Due to this non-trivial topology of band structure, WSMs display a rich variety of exotic transport properties [10, 72], 

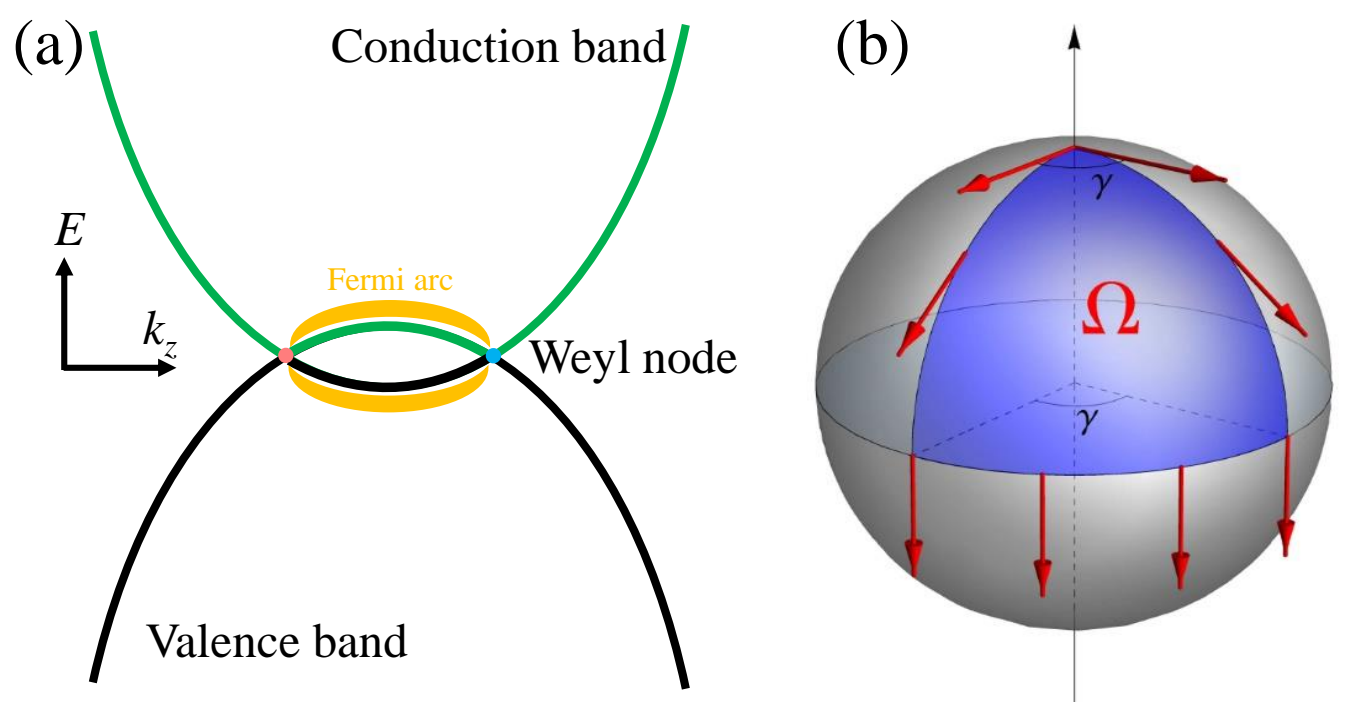

Figure 1.20: (a) The schematic of energy spectrum of a Weyl semimetal. The conduction and valence bands touch at the Weyl nodes with Fermi arcs. $k_{\mathrm{z}}$ is the wave vector. (b) Parallel transport of a vector along a closed path on a sphere.

like negative magnetoresistance, and giant magnitudes of anomalous Hall effect (AHE).

Here, the principle of Berry curvature is briefly introduced. Imagine a general vector that is transported along a closed path on a curved surface, as shown in Figure 1.20(b). If this vector always keeps tangential to the curved surface, its orientation would be changed after the vector came back to its initial point. There is an angle $\gamma$ between the initial and the final vector. The situation is very similar if we consider a quantum state instead of a "classical vector". For a quantum state vector $|\Psi(t)\rangle$ under the action of a Hamiltonian $H(\mathbf{R})$ with an external parameter $\mathbf{R}$, the state vector will accumulate a phase of geometric nature $|\Psi(t)\rangle=e^{i \varphi(t)}|0, t\rangle$ [73] according to the Schrödinger equation, where $|0, t\rangle$ is the instantaneous quantum state at $t$ after the initial quantum state acted with $H(\mathbf{R})$.

$$
\varphi(t)=-\frac{i}{\hbar} \int_{0}^{t} E(t) d t+\gamma
$$


where the first component is dynamic phase, the second component is Berry phase. $\gamma$ can be expressed as a loop $c$ integral in the parameter space $\gamma=\oint_{c} A(\mathbf{R}) d \mathbf{R}$, where $A(\mathbf{R})$ is the Berry connection, thus Berry curvature $\boldsymbol{\Omega}=\nabla \times A(\mathbf{R})$. The Berry curvature can be analogised as a magnetic field in momentum space, which leads to an intrinsic deflection of electrons.

Recently, kagomé-lattice compound $\mathrm{Co}_{3} \mathrm{Sn}_{2} \mathrm{~S}_{2}$ has been proven to be a magnetic WSM with giant anomalous Hall conductivity $\sigma_{\mathrm{xy}} \sim 1130 \Omega^{-1} \mathrm{~cm}^{-1}$ and anomalous Hall angle (a ratio of anomalous Hall conductivity and longitudinal conductivity), 20\% [11], and thin films also exhibit the same transport behaviour as the bulk single crystal [74]. However, $\mathrm{Co}_{3} \mathrm{Sn}_{2} \mathrm{~S}_{2}$ shows a low Curie temperature $T_{\mathrm{c}}=177 \mathrm{~K}$, which means it cannot be used for room temperature devices.

Co-based Heusler compounds, face-centered cubic metallic compounds, have been predicted to be promising candidates to realize magnetic WSMs with high Curie temperature [75, 76]. Bulk crystals of Heusler ferromagnet $\mathrm{Co}_{2} \mathrm{MnGa}$ with large Curie temperature $T_{\mathrm{c}} \approx 700 \mathrm{~K}$ have been studied and shown to display large anomalous Hall angle, $12 \%$, at room temperature, due to a large net Berry curvature near the Fermi energy associated with nodal lines and Weyl points [77, 78, 79]. In addition, Tung et al. [80] and Manna et al. [81], by calculating the Hall conductivity contribution of the majority and minority spin channels from bulk $\mathrm{Co}_{2} \mathrm{MnGa}$, found the spin-up and spin-down Hall currents would flow in opposite directions and the large anomalous Hall conductivity results almost entirely from the majority spin channel. In this case, the anomalous Hall current would be nearly fully spin-polarized, even though $\mathrm{Co}_{2} \mathrm{MnGa}$ is not a half-metallic ferromagnet. The combination of topological electronic properties, ferromagnetism above room temperature, and strongly spin-polarized anomalous Hall current make $\mathrm{Co}_{2} \mathrm{MnGa}$ an exceptional candidate for studying the interplay of topology and magnetism and realizing room temperature topo-spintronics applications, such as magnetic field sensing [82] and generating spin-transfer torque [83]. 


\subsubsection{Skyrmions in Heusler alloys}

Here, the research about skyrmions in Heusler alloys is briefly summarized. Skyrmions have been observed in both bulk and thin film Heusler alloys, but there is very little research. In a non-centrosymmetric bulk Heusler magnet, $\mathrm{Mn}_{1.4} \mathrm{Pt}_{0.9} \mathrm{Pd}_{0.1} \mathrm{Sn}$, skyrmions with $\sim 100 \mathrm{~nm}$ size have been observed by Lorentz transmission electron microscopy [84, 85]. For Heusler alloy thin films sandwiched by a heavy metal and $\mathrm{MgO}$ layer, skyrmions have been observed in $\mathrm{Ta}($ or $\mathrm{Pt}) / \mathrm{Co}_{2} \mathrm{FeAl} / \mathrm{MgO}[86,87]$ and $\mathrm{MgO} / \mathrm{Mn}_{2} \mathrm{CoAl} / \mathrm{Pd}$ [88] heterostructures in the range of 100 to $1000 \mathrm{~nm}$ size due to the interfacial DMI. 


\section{Chapter 2}

\section{Methodology}

This chapter provides an overview of various experimental techniques that have been used in this thesis. Section 2.1 describes thin films prepared by magnetron sputtering. Section 2.2 illustrates how to fabricate devices. Section 2.3 explains the ionic liquid gating technique. Section 2.4 shows different structure characterization methods utilized. Finally, Section 2.5 focuses on the methods that have been used to characterize the magnetic and electrical properties.

\subsection{Thin film growth}

\subsubsection{Substrate cleaning}

Mutilayer thin films are deposited on $10 \times 10 \mathrm{~mm}^{2}$ thermally oxidized $\mathrm{Si} / \mathrm{SiO}_{2}$ $(300 \mathrm{~nm})$ substrates. Before depositing, substrates must be cleaned carefully since they could become contaminated simply after exposing them to the air. Solvents can easily remove oils and organic residues from the surface of $\mathrm{Si}$ substrates, however solvents themselves actually leave residue on the surface of substrates as well. For this reason, a two-solvent method is used to ensure that the substrate is in a contaminant free state. The solvent cleaning method is outlined below: 


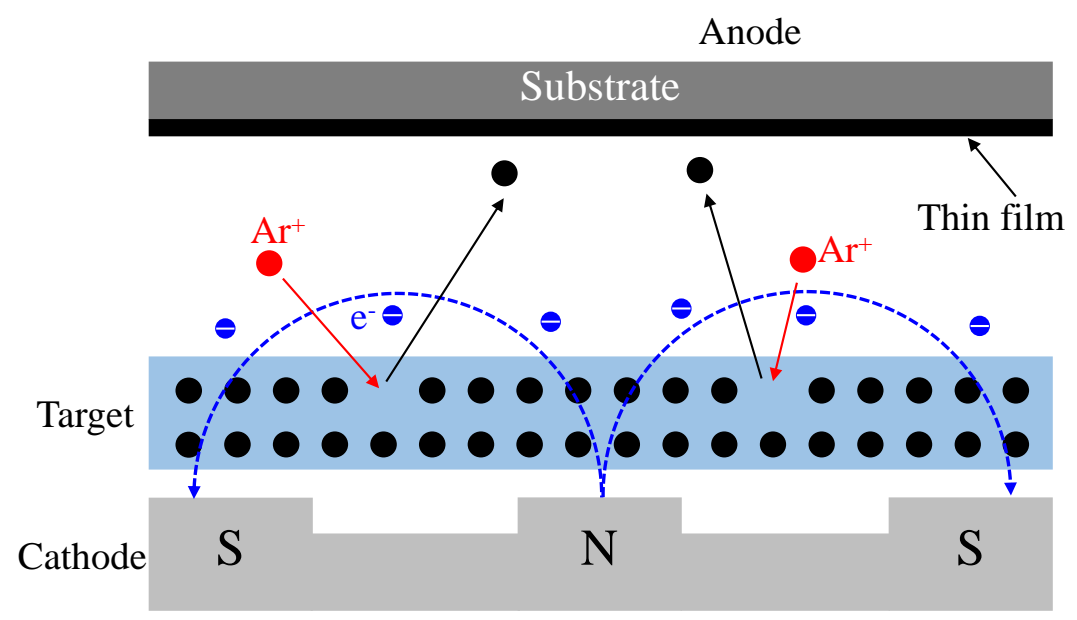

Figure 2.1: Schematic of thin film growth process with magnetron sputtering.

- Soak substrates in acetone for 5 minutes in an ultrasonic cleaner.

- Remove substrates and place them into an isopropyl alcohol container for 5 minutes cleaning.

- Remove substrates from isopropyl alcohol and rinse in deionized water for another 5 minutes cleaning.

- Blow dry substrates with nitrogen.

\subsubsection{Thin film deposition}

Among thin film deposition methods, physical vapour deposition (PVD) is widely used. The processes of PVD happen in a high vacuum environment in which thin films are prepared by evaporating or atomizing a solid target source in the working chamber. In this thesis, a magnetron sputtering system, a type of PVD system, was used to prepare insulating or metallic thin films.

The basic sputtering process is as follows (see Figure 2.1). A sputtering gas (e.g. Ar) is introduced into a vacuum chamber at a working pressure of 1 to 100 mTorr. Then, a DC voltage is applied between the target and 

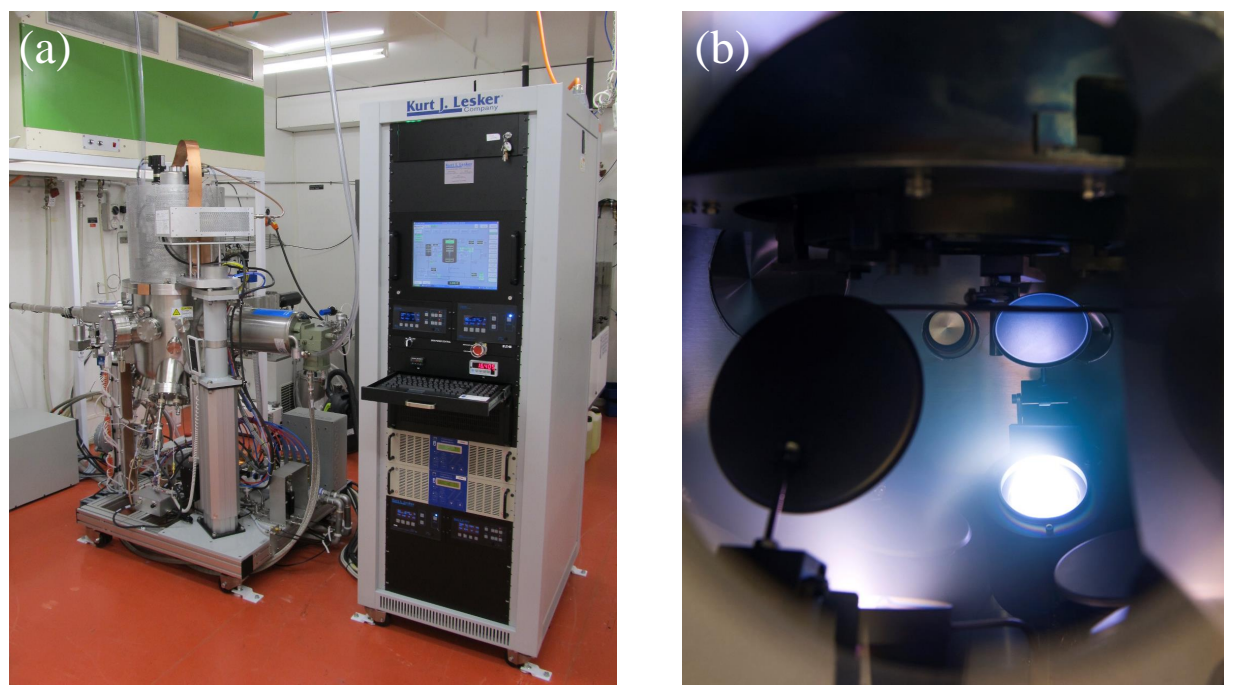

Figure 2.2: (a) CMS-18 magnetron sputtering system (Robinson Research Institute) and (b) flux of plasma.

substrate which ionizes the Ar atoms and creates a plasma in the chamber. These Ar ions are accelerated to the target and cause atoms from the target to be ejected. Finally, these ejected atoms travel through the high vacuum environment until they reach the substrate and form a thin film. For magnetron sputtering, there is a strong magnetic field near the target area. This magnetic field forces electrons from the process of Ar ionization along magnetic flux lines near the surface of the target instead of being attracted toward the substrate so that the plasma is confined to an area near the target, without causing damage to the thin film being formed. Also, electrons confined to the surface of target could increase the probability of further ionizing Ar atoms so that more target atoms can be ejected, and therefore the efficiency of the sputtering process can be increased.

A CMS-18 sputtering system produced by the Kurt J. Lesker company (www.lesker.com) (see Figure 2.2) was used. This thin film deposition system is equipped with six targets, substrate heater which can go up to 550 ${ }^{\circ} \mathrm{C}$, and both RF and DC power supplies. Before deposition, the substrate surface can be cleaned by the $\mathrm{Ar}^{+}$plasma. The pressure of the main cham- 


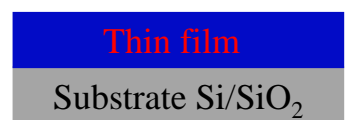

(a)

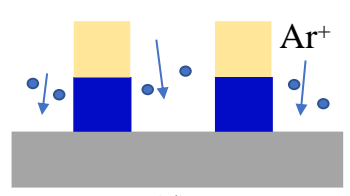

(d)

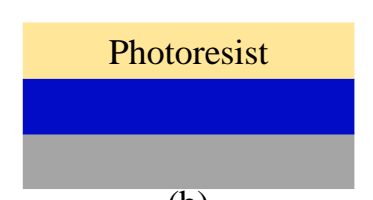

(b)

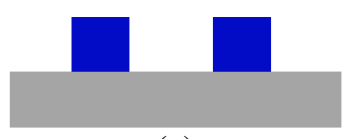

(e)

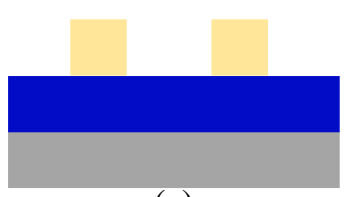

(c)

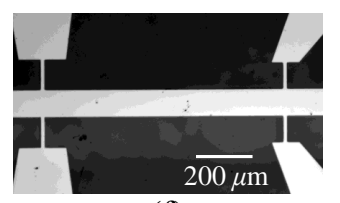

(f)

Figure 2.3: The processes of device fabrication. (a) Thin film deposition; (b) Spin coating photoresist; (c) Exposing and developing photoresist into the device pattern; (d) Ar milling; (e) Final device; (f) An example device measured by optical microscopy.

ber can reach to below $3 \times 10^{-8}$ Torr by using the cryo-pump. The typical growth pressures for the films of this thesis are 2 to 10 mTorr.

\subsection{Device fabrication}

In this work, a dry etching method was used to fabricate devices. The processes are outlined below:

- Deposit thin film on the substrate (Figure 2.3(a));

- Spin coat the negative photoresist AZ2070 on the thin film with a $4000 \mathrm{rpm}$ speed, and then soft bake it for $90 \mathrm{~s}$ at $110{ }^{\circ} \mathrm{C}$ on a vacuum hotplate (Figure 2.3(b));

- Expose the photoresist in the laser writer, and then soft bake it for 90 $\mathrm{s}$ at $100{ }^{\circ} \mathrm{C}$ on the vacuum hotplate;

- Place the sample in the developer AZ726 MIF for 4 minutes (Figure 2.3(c));

- Etch the sample by ion milling (Figure 2.3(d)); 
(a)
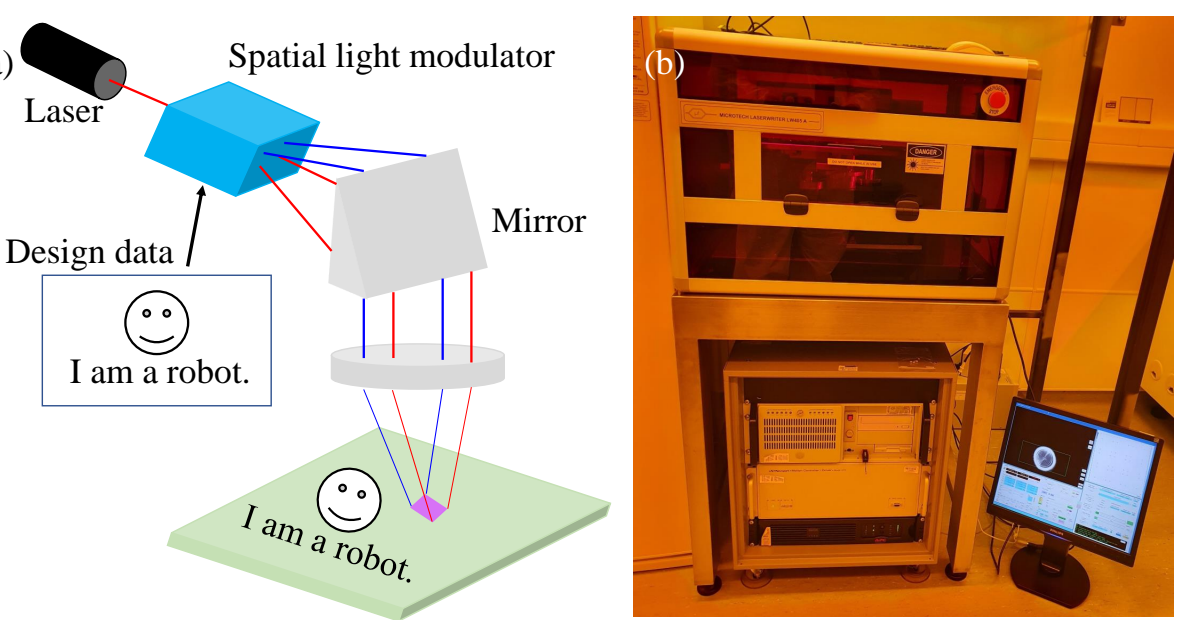

Figure 2.4: (a) Schematic of laser writer. "I am a robot" is an example of the designed pattern structure. (b) Microtech Lw405 model (Callaghan Innovation, Wellington).

- Remove the photoresist that remains on top of the patterned material with acetone, and then rinse in deionized water and blow dry the device with nitrogen (Figure 2.3(e)).

Figure 2.3(f) shows a patterned Hall bar structure with a $100 \mu \mathrm{m}$ width as an example.

\subsubsection{Laser writer lithography}

Laser writer lithography is a high resolution maskless lithography method, able to pattern features down to sub-micron line width, for direct writing on any flat surface coated with photoresist. It transforms a focused laser beam into a spatial light modulator which serves as a programmable mask, then patterns are generated by accurately moving the substrate underneath a focused and scanning laser beam with proper wavelength, as shown in Figure 2.4(a). In this thesis the laser writer used is Microtech LW405 model (Figure 2.4(b)), in the Callaghan Innovation yellow room. 


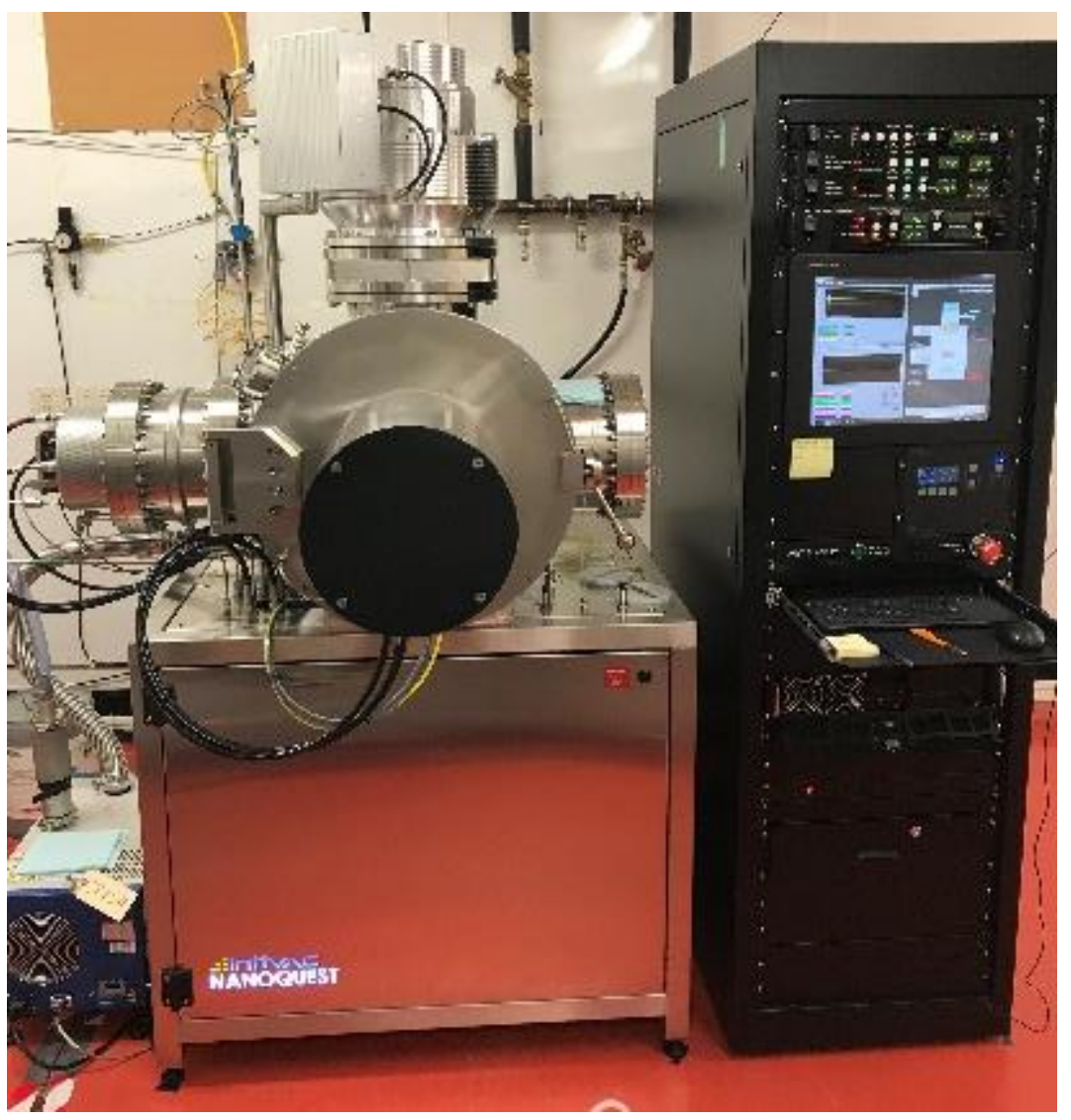

Figure 2.5: Ion milling (Robinson Research Institute).

\subsubsection{Ion milling}

Ion milling is a purely physical process employed for cleaning the surface or etching away material on a substrate. During the etching processes, Ar ions are accelerated to the surface of the sample and mill away the material that is not protected with a covering photoresist. In this work, Ar ions are perpendicular to the $10 \times 10 \mathrm{~mm}^{2}$ sample and accelerated to create a beam with a $300 \mathrm{~V}$ beam voltage, $110 \mathrm{~mA}$ beam current and 45 $\mathrm{V}$ accelerator voltage for etching 5 minutes under $2.3 \times 10^{-4}$ Torr at $10^{\circ} \mathrm{C}$. The ion milling system (Intlvac) is used in the Robinson Research Institute thin film lab, as shown in Figure 2.5. 
(a)

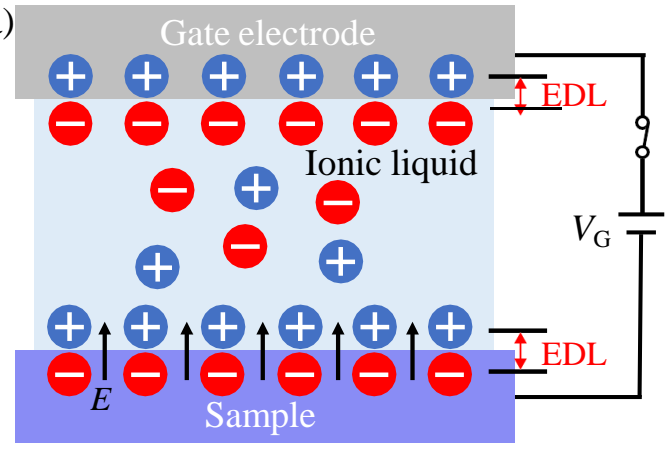

(b)

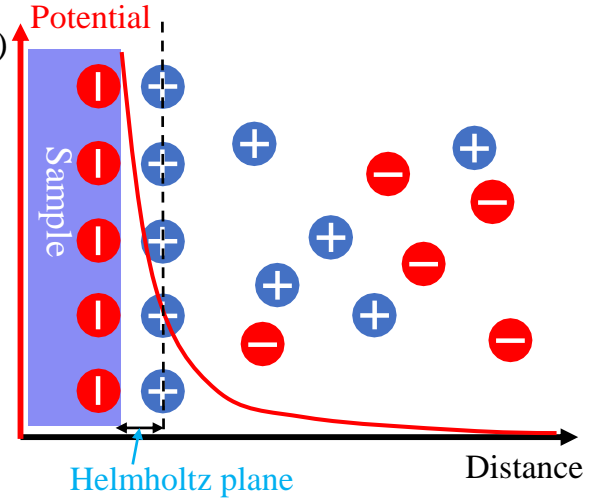

Figure 2.6: (a) Schematic of electric double layer (b) The decay of the electrical potential (red line) in the electric double layer.

\subsection{Ionic liquid gating}

Ionic liquid gating (ILG) is a method using ionic liquids or ion gels to form an electric double layer (EDL) under a low voltage, in which an high electric field (the order of hundreds of $\mathrm{MV} / \mathrm{cm}$ ) can be generated at the EDL interface over a nanometer scale gap [89], as shown in Figure 2.6.

This interfacial gating behaviour can lead to controlled and reversible changes of carrier concentration, and to a magneto-ionic effect [90]. It can thus achieve substantial modifications of magnetic anisotropy [91, 92] and DMI [93]. In this thesis, a small droplet of ionic liquid, N,N-Diethyl-Nmethyl-N-(2-methoxyethyl)ammoniumbis-(trifluoromethylsulfonyl)imide (DEME-TFSI) (IoLiTec), is used as the electrolyte connecting the Pt wire gate electrode and the surface of sample. In order to obtain a uniform thickness a piece of glass cover slide (100 $\mu \mathrm{m}$ thick) is placed on the ionic liquid. This ensures an aberration free optical path for the optical measurement. Samples are charged for 5 minutes at each voltage to reach the equilibrium of carriers. The gate voltage is applied by a Keithley 2450 source meter. 
(a) Incident X-rays

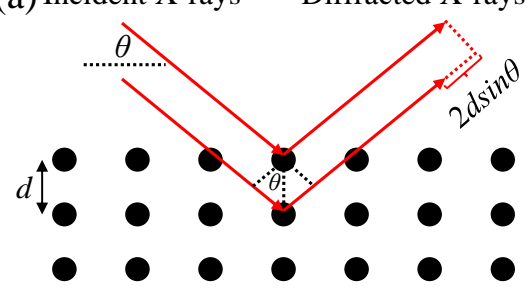

(b)

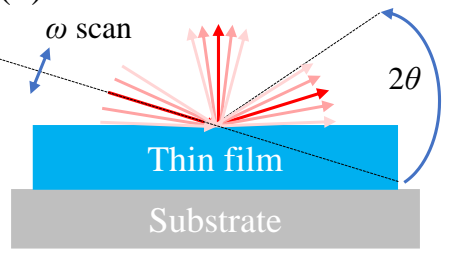

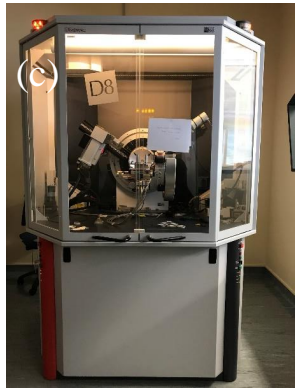

Figure 2.7: (a) Schematic of XRD and (b) rocking curve geometry; (c) Bruker D8 XRD system

\subsection{Structure characterization}

In this research, a variety of techniques have been used to characterize the thickness, composition, roughness and crystal structure of thin films. Knowing this information will allow us to optimize the growth condition and then improve the quality of samples which determines the electrical and magnetic properties. The crystal structure can be detected by $X-$ ray diffraction (XRD). The thickness can be measured by $X$-ray reflectivity (XRR) and scanning electron microscopy (SEM). The energy-dispersive $X-$ ray spectroscopy (EDX) can be used to characterize the stoichiometry of the Heusler alloys. The surface roughness is a important parameter for ultrathin films, and it can be measured by atomic force microscopy (AFM) and XRR. The chemical states at the surface is characterized by X-ray photoelectron spectroscopy (XPS).

\subsubsection{X-ray diffraction and X-ray reflectivity}

When monochromatic X-rays irradiate the surface of a sample, scattered beams from parallel lattice planes will undergo a constructive interference. This constructive diffraction can be described by Bragg's Law

$$
n \lambda=2 d \sin \theta,
$$



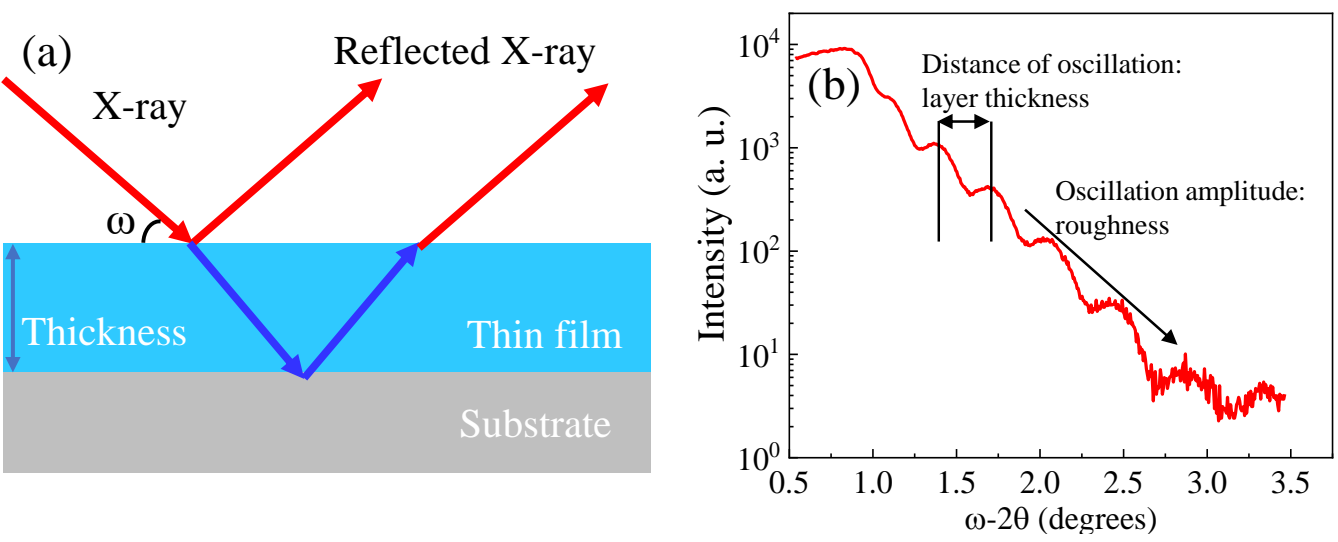

Figure 2.8: (a) Schematic of XRR geometry and (b) an experimental example of 20 $\mathrm{nm} \mathrm{Co}_{2} \mathrm{MnGa}$ thin film.

where $\lambda$ is the $\mathrm{X}$-ray wavelength, $\theta$ is the incidence angle, $d$ is the distance between parallel planes and $n$ is the order of reflection (see Figure 2.7(a)). In this work, the crystal structure of thin films is characterized by using a powder diffractometer (Bruker D8) with Co $K_{\alpha}$ radiation $(\lambda=1.7889 \AA)$ at Callaghan Innovation.

It is well known that the crystal orientation or texture and the degree of orientation are two important factors that can strongly affect the physical properties of a thin film. The crystal orientation can be determined by a symmetrical reflection measurement, as shown in Figure 2.7(a), and the degree of distribution of crystal orientation can be obtained by a rocking curve measurement, as shown in Figure 2.7(b). This method is performed by rocking the thin film sample while the detector is kept a fixed $2 \theta$ angle to detect diffraction intensity from the preferentially-oriented lattice planes. The distribution of crystal orientation is estimated from the full width at half maximum (FWHM) of the rocking curve profile.

From XRR, one can obtain thin film parameters including thickness, density and surface and interface roughness. When $X$-rays are irradiated onto a surface and interface of thin film, interference occurs between the $X$-rays reflected from the surface of the thin film and the interface between 
the thin film and substrate, as shown in Figure 2.8(a), the reflectivity profile shows oscillations caused by this X-ray interference. The oscillations are caused by the different phase difference between the $\mathrm{X}$-rays reflected from the different interfaces, and their period is dependent on the distance between the surface and interface, in other words, film thickness. Figure 2.8(b) shows a typical reflectivity curve for a single layer thin film and the corresponding information obtained from this oscillation measured by PANalytical X'Pert PRO. By fitting this curve and calculating the thickness, one can obtain the growth rate of thin films with deposition time.

\subsubsection{Scanning electron microscopy and energy-dispersive X-ray spectroscopy}

SEM is a microscopy technique which can be used to measure a high resolution $(10 \mathrm{~nm})$ image about the morphology of the sample. When a sample is illuminated with a focused high energy electron beam, secondary and back-scattered electrons can be emitted from the sample surface. Topography of the surface can be observed by two-dimensional scanning of the electron probe over the surface and acquisition of an image from the detected secondary or back-scattered electrons. Also, the thickness of a thin film can be measured by imaging the cross section, therefore, the growth rate can be calculated by using the deposition time.

EDX is an analytical technique used to identify the elemental composition of materials. Firstly, using a high energy electron beam causes electrons from inner atomic shells of the sample to excite. Then, higher state electrons will fill the vacancies and release X-rays, as shown in Figure 2.9(a). Since the energy of $X$-rays released during this process is unique to each element on the periodic table, an energy-dispersive spectrometer can be used to detect the emitted X-rays and identify what elements are present and in which corresponding proportions. Figure 2.9(b) shows an example of EDX-spectrum of a $20 \mathrm{~nm} \mathrm{Co}{ }_{2} \mathrm{MnGa}$ thin film measured by 

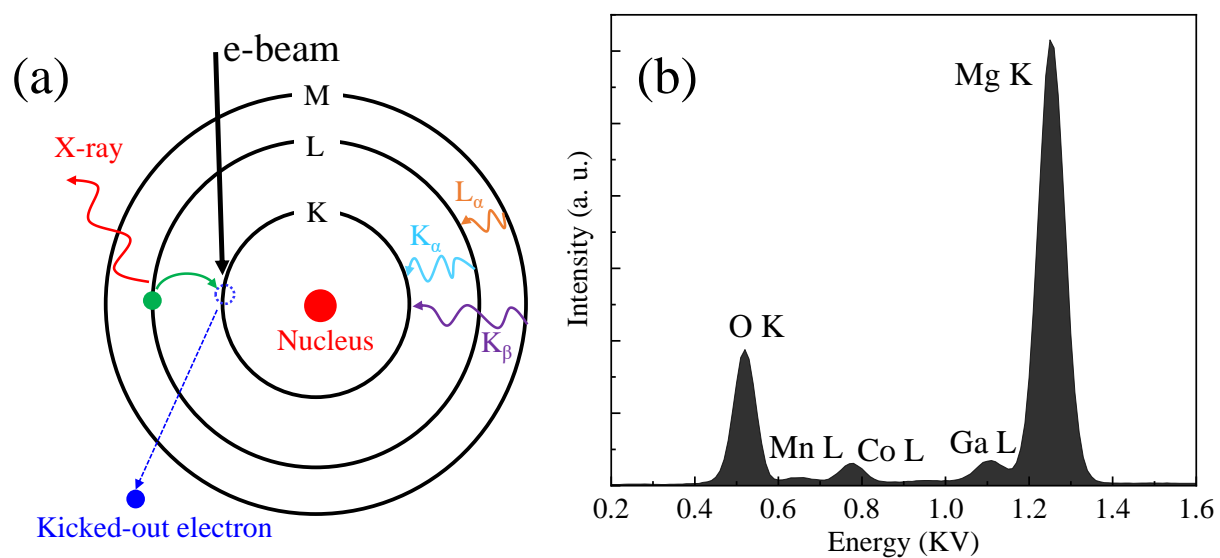

Figure 2.9: (a) Schematic of EDX (b) an experimental spectrum of $\mathrm{Co}_{2} \mathrm{MnGa}$ thin film on $\mathrm{MgO}$ substrate.

Quanta SEM.

\subsubsection{Atomic force microscopy}

AFM is another imaging technique in which both the vertical and lateral resolution can reach the order of $\AA$ [94] by using a cantilever with a very sharp tip to scan over a sample surface. Thus, it can be used to accurately characterize the roughness of thin films. The AFM relies on the force between the tip and sample which can be calculated by Hooke's law:

$$
F=-k z
$$

where $k$ and $z$ are the stiffness and deflection of cantilever, respectively. As the tip approaches the surface, it forms an attractive force between the surface and the tip when it draws closer to the sample surface. However, a repulsive force gradually takes control making the cantilever avert from the surface when the tip is very close to the surface of the sample, as shown in Figure 2.10(b). During this scanning process, a laser beam is used to detect the cantilever deflection towards or away from the surface by reflecting an incident beam off the flat top of the cantilever, as shown 


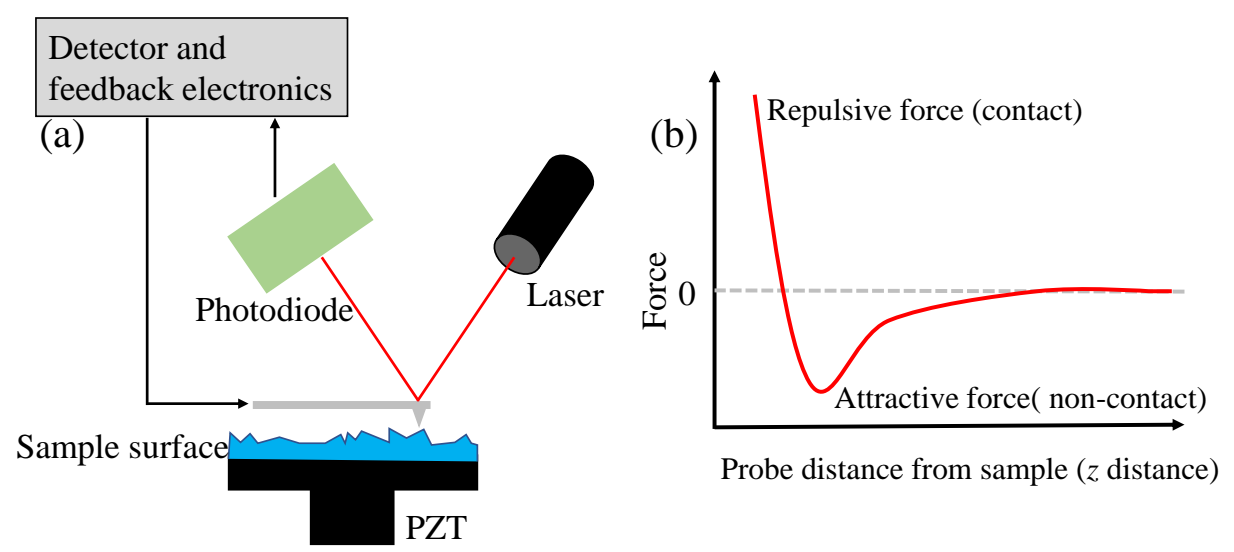

Figure 2.10: (a) Schematic of AFM and (b) force curve as a function of $z$ distance.

in the Figure 2.10(a). A position-sensitive photodiode can be used to track and record these deflection changes. By using a feedback loop to control the height of the tip above the surface while maintaining a constant laser position, an accurate topographic image of the sample can be obtained.

\subsubsection{X-ray photoelectron spectroscopy}

XPS is used to explore the elemental composition and chemical states of the first few atomic layers at the surface. Surface analysis by XPS involves irradiating a sample with monoenergetic $X$-rays and analyzing the energy of the emitted photoelectrons. The X-ray photons interact with atoms in the surface region, causing electrons to be emitted by the photoelectric effect, as shown in Figure 2.11. The kinetic energy of the emitted electrons is given by:

$$
K E=h \nu-B E-\phi,
$$

where $h \nu$ is the energy of the photon, $B E$ is the binding energy, and $\phi$ is the work function. The binding energy is regarded as the energy difference between the initial state of the core level and the Fermi level after the photoelectron has left the atom. Because each element has a unique set of binding energies, XPS can be used to identify and determine the elemen- 


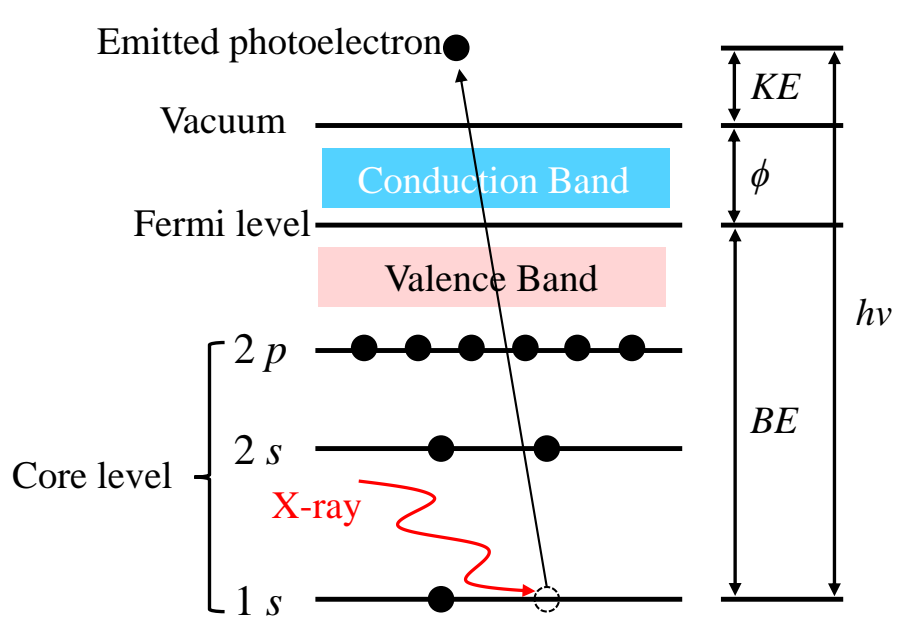

Figure 2.11: (a) Schematic of XPS principle.

tal composition and chemical states at the surface of thin films. The Kratos Axis Ultra DLD system with a monochromatic $\mathrm{Al} \mathrm{K}_{\alpha} \mathrm{X}$-ray source in the University of Auckland is used in this work.

\subsection{Electrical and magnetic characterization}

Electrical measurements are undertaken with a Quantum Design physical properties measurement system (PPMS). It is a system for the measurement of material properties, like magnetic, electrical and thermal transport properties, which is capable of achieving temperature from 1.9 to 400 $\mathrm{K}$ and magnetic field up to $9 \mathrm{~T}$. Magnetic measurements are performed by using superconducting quantum interference device (SQUID) magnetometer allowing extremely sensitive magnetic measurements in a magnetic field of up to $7 \mathrm{~T}$ and at temperature down from 1.9 to $400 \mathrm{~K}$. A magneto-optical Kerr effect (MOKE) microscope is used to image the magnetic domains and also measure the hysteresis loop in a magnetic field up to $1 \mathrm{~T}$ along both the perpendicular and longitudinal direction. 

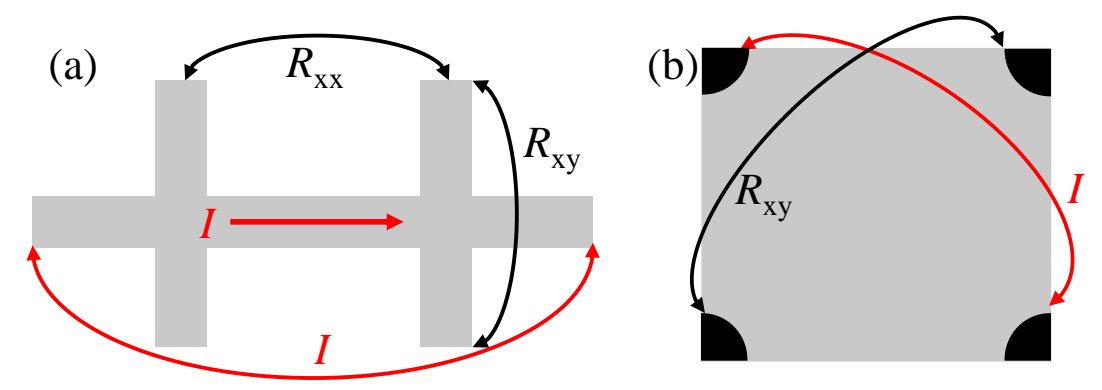

Figure 2.12: (a) Four-wire resistance geometry and (b) Van der Pauw geometry.

\subsubsection{Physical properties measurement system}

In this thesis, the resistivity option of PPMS is employed to measure the longitudinal $\left(R_{\mathrm{xx}}\right)$ and Hall $\left(R_{\mathrm{xy}}\right)$ resistance with the standard four-wire or Van der Pauw geometry, as shown in Figure 2.12. Thin films are mounted on resistivity pucks and placed on the horizontal rotator which enables a sample to be rotated over a full $360^{\circ}$ in the presence of an applied magnetic field (up to $\pm 9 \mathrm{~T}$ ) and a controlled temperature $(1.8$ - $400 \mathrm{~K}$ ) (see Figure 2.13). The temperature sensor on the rotator is underneath the puck so that the measured temperature is very close to that of samples. The AC drive mode was used in this thesis. In AC mode, the user bridge board applies a DC current to the sample and reads the potential drop across the sample. Then the user bridge board reverses the current and reads the potential drop again. The user bridge averages the absolute value of the positive and negative voltage readings. Operation in AC mode eliminates errors from DC offset voltages and produces the most accurate readings. A 1 - $100 \mu \mathrm{A}$ DC current is applied to perform the electrical transport measurement, like anomalous Hall effect (AHE), in this thesis.

AHE describes the fact that when an electric current flows through a ferromagnetic metal with an out-of-plane magnetization, the electrons with opposite spin direction would accumulate at the transverse direction and induce a large Hall voltage [96], as shown in Figure 2.14(a). Usually, the Hall resistivity is comprised of two terms, 


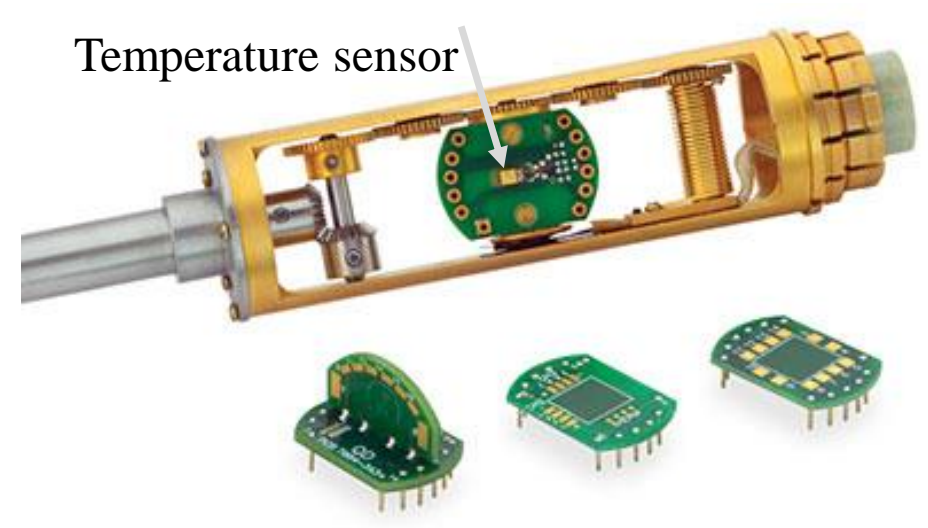

Figure 2.13: Top: horizontal rotator, and bottom: resistivity pucks with different function [95].

$$
\rho_{\mathrm{xy}}=R_{\mathrm{o}} H_{\mathrm{z}}+R_{\mathrm{s}} M_{\mathrm{z}}
$$

where $R_{\mathrm{o}}$ and $R_{\mathrm{s}}$ are the ordinary and anomalous Hall coefficient, respectively. In ferromagnetic materials, $R_{\mathrm{S}}$ is much larger than $R_{\mathrm{o}}$ so that the $\rho_{\mathrm{AH}}$ is proportional to the $M_{\mathrm{z}}$.

There are three mechanisms that can give rise to an anomalous Hall effect: intrinsic deflection, side jump and skew scattering [96]. The intrinsic contribution originates from the momentum-space Berry curvature which induces an electric field and leads to an anomalous velocity of electrons, as shown in Figure 2.14(b). For the side jump, the electron velocity is deflected in opposite directions when electrons approach and leave an impurity (Figure 2.14(c)). The skew scattering is simply related to the transport lifetime, which dominates in nearly perfect crystals (Figure 2.14(d)). The presence of skew scattering is due to the spin-orbit coupling from the disorder or electron of ferromagnet.

Experimentally, three distinct regimes have been identified [97]: (i) a high conductivity regime, $\sigma_{\mathrm{xx}}>10^{6} \Omega^{-1} \mathrm{~cm}^{-1}$, in which a linear contribution to $\sigma_{\mathrm{xy}} \sim \sigma_{\mathrm{xx}}$ due to skew scattering dominates; (ii) an intrinsic regime, $10^{4} \Omega^{-1} \mathrm{~cm}^{-1}<\sigma_{\mathrm{xx}}<10^{6} \Omega^{-1} \mathrm{~cm}^{-1}$, in which $\sigma_{\mathrm{xy}}$ is nearly a con- 


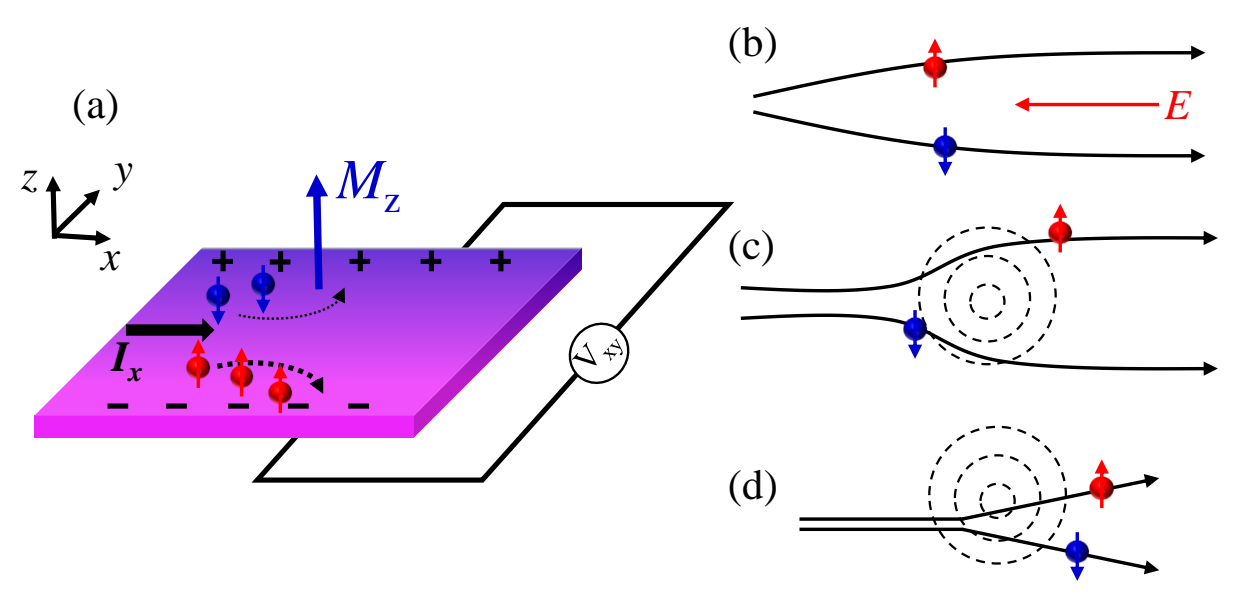

Figure 2.14: (a) Schematic of AHE. Mechanism of AHE, (b) intrinsic deflection, (c) side jump, and (d) skew scattering.

(a) Plastic straw
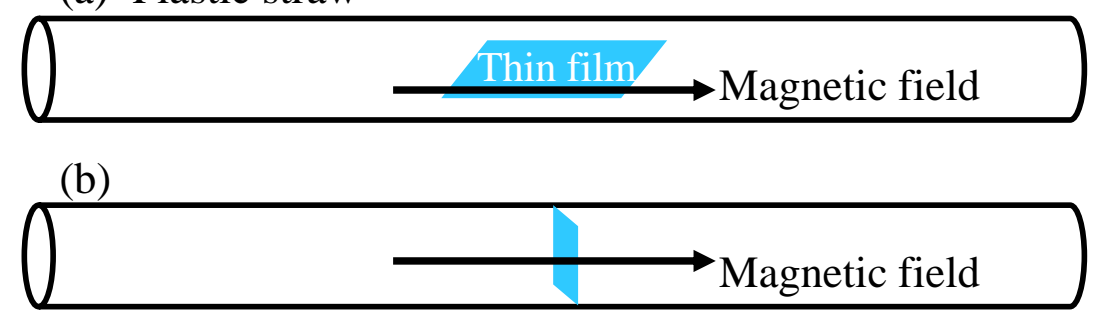

Figure 2.15: (a) Parallel and (b) perpendicular geometry.

stant of $1000 \Omega^{-1} \mathrm{~cm}^{-1}$; and (iii) a dirty regime $\sigma_{\mathrm{xx}}<10^{4} \Omega^{-1} \mathrm{~cm}^{-1}$ in which $\sigma_{\mathrm{xy}} \sim \sigma_{\mathrm{xx}}^{1.6}$.

If the magnetization in Figure 2.14(a) is along the in-plane direction, a small transverse resistivity can be measured which is called planar Hall effect [98]. This effect originates from the interaction between the magnetization and current in the plane of the film. 


\subsubsection{Superconducting quantum interference device mag- netometer}

SQUID magnetometry is a technique to record the change of magnetic flux by SQUID sensors and so measure the magnetic properties of materials at a certain temperature and magnetic field. A SQUID device is the most sensitive device available for measuring magnetic field or magnetic flux that approaches $10^{-15} \mathrm{~T}$ [99]. It consists of a closed superconducting loop including one or two Josephson junctions in the loop's current path. In this thesis, the reciprocating sample option (RSO) module of a Quantum Design MPMS-XL SQUID magnetometer is employed to measure the magnetization of thin films at an external magnetic field (up to $\pm 7 \mathrm{~T}$ ) and a controlled temperature $(1.8-400 \mathrm{~K})$. The sample is mounted in a plastic straw with two different geometries that the magnetic field is parallel to or perpendicular to the surface of thin film so that the magnetic anisotropy can be confirmed, as shown in Figure 2.15. It should be noted that a standard sample mounting method is to place samples in a plastic straw to minimize background contribution. The RSO option measures the magnetization by moving the sample rapidly and sinusoidally through the SQUID pickup coils. The moving magnetic moment induces an electric current in the coils which are inductively coupled to a SQUID sensor generating a output SQUID voltage, so that the magnetization signal with a sensitive of $10^{-8}$ emu would be converted to measurable a voltage (see Figure 2.16).

\subsubsection{Magneto-optical Kerr effect microscope}

\section{Principle}

The magneto-optical Kerr effect (MOKE) describes the change of the polarization of light when reflected from a surface of a magnetized medium. It is well known that a linearly polarized light is an electromagnetic wave, which can be formed by a superposition of left and right circularly polar- 


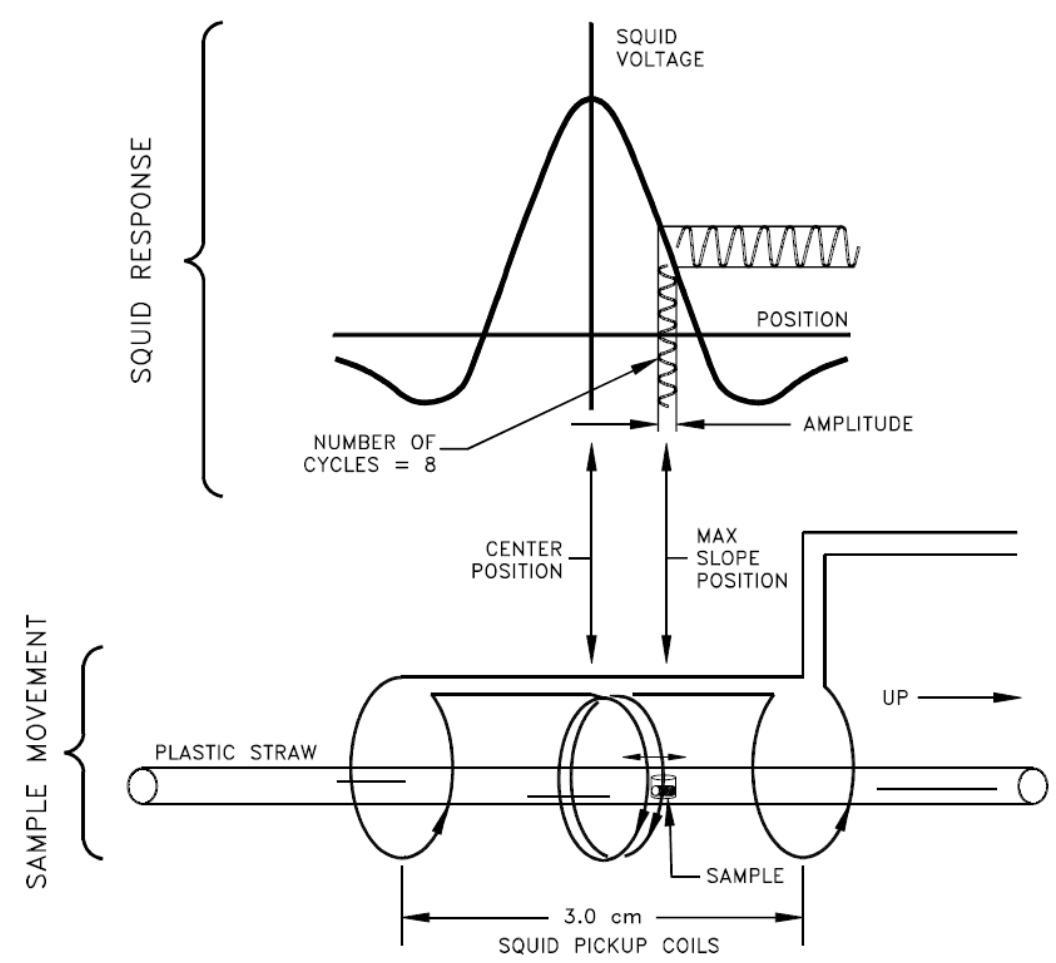

Figure 2.16: Schematic of the RSO measurement of the SQUID magnetometer [100]. The W-shape curve represents the signal for an ideal magnetic dipole detected by the SQUID coils as a function of position.

ized components. For MOKE, there are two processes happening when a linearly polarized light penetrates into a magnetized medium, as shown in Figure 2.17(a). First, the two circularly polarized components obtain different phase shifts due to their different propagating velocities, which leads to a rotation of the polarization plane called Kerr rotation $\left(\theta_{\mathrm{k}}\right)$. Second, the different absorption rates of the medium for the two circularly polarized components affect the amplitude of both components and thus induce a Kerr ellipticity $\left(\eta_{\mathrm{k}}\right)$. These two parameters form a Kerr angle

$$
\phi_{\mathrm{k}}=\theta_{\mathrm{k}}+i \eta_{\mathrm{k}} .
$$

Normally, $\theta_{\mathrm{k}}$ and $\eta_{\mathrm{k}}$ are proportional to the magnetization of magnetic materials. Therefore, MOKE can be used to investigate the magnetic proper- 
(a)

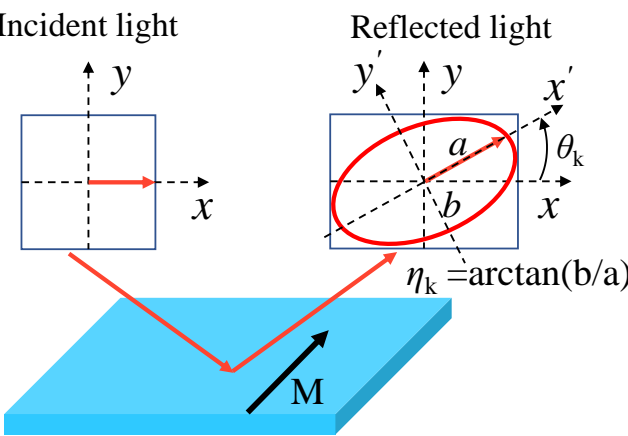

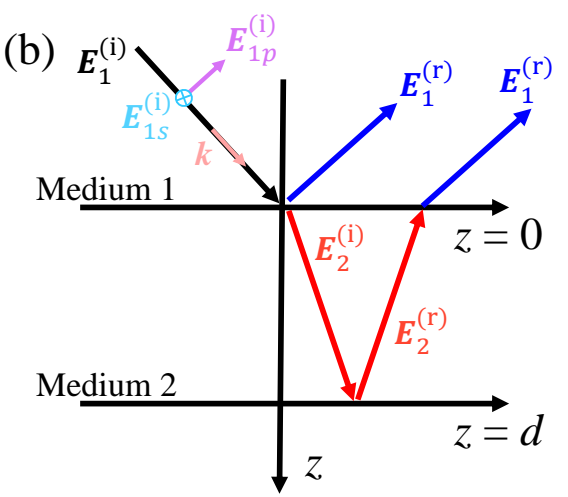

Figure 2.17: (a) Schematic of MOKE. (b) The change of electric field of polarized light after propagating into medium 2. $E_{1(2)}^{(\mathrm{i})}$ and $E_{1(2)}^{(\mathrm{r})}$ represent the incident and reflected light, respectively, in medium 1 or medium $2 . E_{1 \mathrm{~s}}^{(\mathrm{i})}$ and $E_{1 \mathrm{p}}^{(\mathrm{i})}$ represent the direction of electric field of incident light perpendicular and parallel to the plane of incidence, respectively. $k$ is the wave vector.

ties of the surface of ferromagnetic thin films.

The Kerr angle can be calculated by using electromagnetic theory [101]. Considering an electromagnetic wave $E=E_{0}\left(e^{i \mathbf{k} \cdot \mathbf{r}-i \omega t}\right)$ that propagates into a medium whose dielectric tensor is

$$
\varepsilon=\varepsilon_{0}\left(\begin{array}{ccc}
1 & i Q_{z} & -i Q_{y} \\
-i Q_{z} & 1 & i Q_{x} \\
i Q_{y} & -i Q_{x} & 1
\end{array}\right)
$$

where $Q_{x, y, z}$ is the magneto-optical constant or Voigt constant. Figure 2.17(b) shows the schematic of the incident and reflected light in MOKE measurement. The amplitude of electric field of the reflected light can be expressed in matrix notation

$$
\left(\begin{array}{c}
E_{p}^{(\mathrm{r})} \\
E_{s}^{(\mathrm{r})},
\end{array}\right)=R\left(\begin{array}{c}
E_{p}^{(\mathrm{i})} \\
E_{s}^{(\mathrm{i})},
\end{array}\right),
$$

where $R$ is the magneto-optical Fresnel reflection matrix. By solving Maxwell's equations for that $\varepsilon, R$ can be obtained

$$
R=\left(\begin{array}{cc}
r_{p p} & r_{p s} \\
r_{s p} & r_{s s}
\end{array}\right)
$$




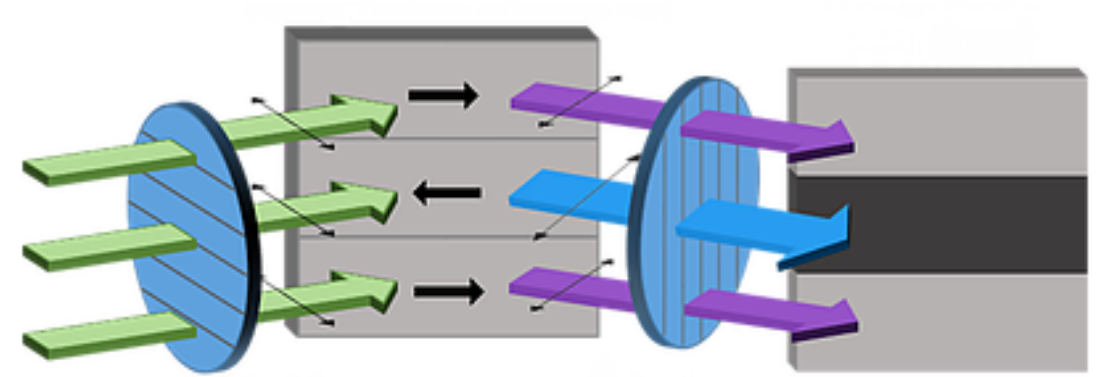

Figure 2.18: Schematic of MOKE. A linearly polarized light illuminates a magnetic material, then there is a contrast for those domains with opposite direction of magnetic moments.

where $s$ and $p$ relate to the $s$ - and $p$-polarized components of the incident wave, respectively. The definitions of the magneto-optical coefficients are

$$
r_{p p}=\frac{E_{1 \mathrm{p}}^{(\mathrm{r})}}{E_{1 \mathrm{p}}^{(\mathrm{i})}}, r_{s p}=\frac{E_{1 \mathrm{~s}}^{(\mathrm{r})}}{E_{1 \mathrm{p}}^{(\mathrm{i})}}, r_{p s}=\frac{E_{1 \mathrm{~s}}^{(\mathrm{r})}}{E_{1 \mathrm{p}}^{(\mathrm{i})}}, r_{s s}=\frac{E_{1 \mathrm{~s}}^{(\mathrm{r})}}{E_{1 \mathrm{~s}}^{(\mathrm{i})}} .
$$

$r_{p p}, r_{s p}$ and $r_{p s}$ include a term depending on Voigt constant. It should be noted that some of the reflected $E_{1}^{(\mathrm{r})}$ are induced from the transmission of $E_{2}^{(\mathrm{r})}$ which carry the information of magnetization of medium 2. The Kerr angle for $s$ - and $p$ - polarized light are given by

$$
\phi_{\mathrm{k}(\mathrm{s})}=\theta_{\mathrm{k}(\mathrm{s})}+i \eta_{\mathrm{k}(\mathrm{s})}=\frac{r_{p s}}{r_{s s}} \text { and } \phi_{\mathrm{k}(\mathrm{p})}=\theta_{\mathrm{k}(\mathrm{p})}+i \eta_{\mathrm{k}(\mathrm{p})}=\frac{r_{s p}}{r_{p p}}
$$

Therefore, by measuring the amplitude of $E$ of the reflective light with a photo-diode, one can obtain the the Kerr angle.

MOKE microscopy is a powerful tool for studying the magnetic morphology of ferromagnetic thin films. In Figure 2.18, the illuminating light is first passed through a polarizer, then reflects from the sample and passes through an analyser polarizer, before going through a regular optical microscope or camera. The changes in polarization are converted by the analyser into changes in light intensity, which are visible. 
(a)

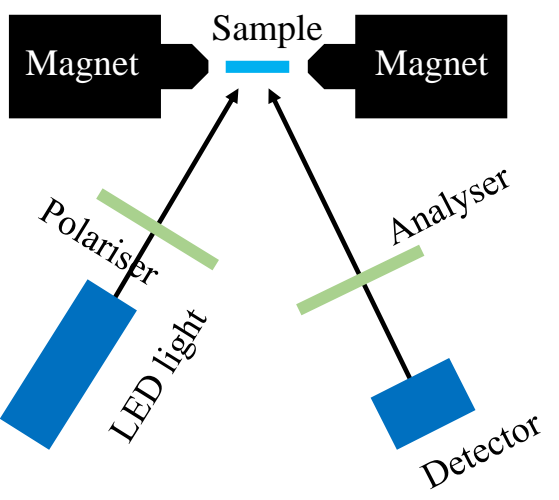

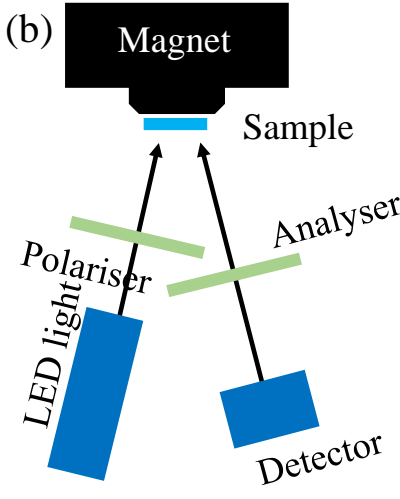

Figure 2.19: (a) Longitudinal and (b) polar MOKE configurations.

\section{Geometry}

There are three geometries for MOKE measurement, the longitudinal, transverse, and polar geometries, which are classified with respect to the direction of the magnetization vector of the film, the plane of light incidence and the sample surface. In this thesis, two of those are used in MOKE system.

- Longitudinal Kerr effect: in which the magnetization vector is parallel to both the plane of the incident light and the sample surface for measuring the in-plane component of magnetization (see Figure 2.19(a)).

- Polar Kerr effect: in which the magnetization field is parallel to the plane of the incident light but perpendicular to the sample plane. In this configuration only the out-of-plane component of magnetization is measured (see Figure 2.19(b)).

\section{$K_{\text {eff }}$ calculation}

The MOKE system used in this thesis is equipped with a combinatorial electromagnet which can generate both in-plane and out-of-plane magnetic fields. Therefore, the effective magnetic anisotropy, $K_{\text {eff }}$, can be mea- 

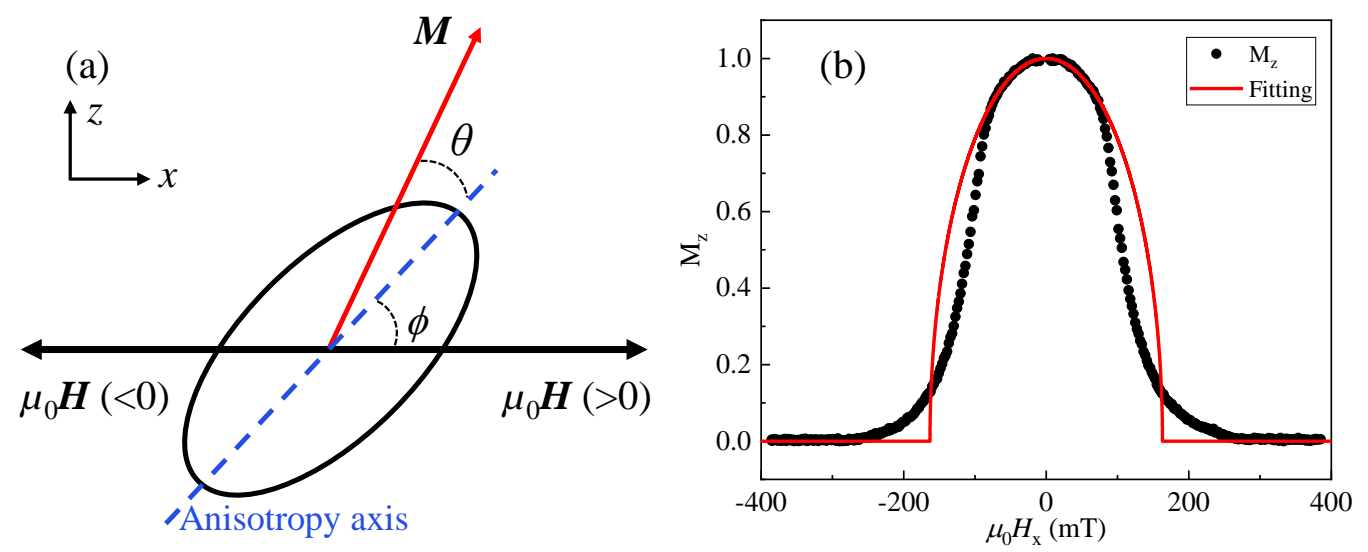

Figure 2.20: (a) Schematic of Stoner-Wohlfarth model for a single domain. (b) Rotation of magnetization from out-of-plane to in-plane direction by sweeping the in-plane magnetic field for $\mathrm{MgO}(1.6 \mathrm{~nm}) / \mathrm{Mn}_{2} \mathrm{CoAl}(2.6 \mathrm{~nm}) / \mathrm{Pd}(3.2 \mathrm{~nm})$ trilayer.

sured by using the Stoner-Wohlfarth model. This model describes the physics of magnetic grains with single domains under an applied field in which the magnetization reverses coherently [102]. For a single domain with an ellipsoid form (see Figure 2.20(a)), the total energy consisting of the anisotropy energy $E_{\mathrm{A}}$ and the Zeeman energy $E_{\mathrm{ze}}$ is

$$
E=E_{\mathrm{A}}+E_{\mathrm{ze}}=K_{\mathrm{u}} \sin ^{2} \theta-\mu_{0} M_{\mathrm{s}} H \cos (\theta-\phi),
$$

where $K_{\mathrm{u}}$ is the uniaxial anisotropy, $\theta$ is the angle between the $M$ and anisotropy axis, $\phi$ is the angle between the external magnetic field and anisotropy axis. It should be noted that $E_{\mathrm{A}}$ is determined from all contributing energy terms as mentioned in section 1.5 except Zeeman energy so that the magnetization lies along the anisotropy axis (easy axis) without external magnetic field.

Here, an example of the $K_{\text {eff }}$ measurement by MOKE is gave. For the $\mathrm{MgO} / \mathrm{Mn}_{2} \mathrm{CoAl} / \mathrm{Pd}$ trilayer with an anisotropy axis along out-of-plane direction ( $z$ axis), the magnetization will rotate coherently following the Stoner-Wohlfarth model by applying an in-plane magnetic field $\mu_{0} H_{\mathrm{x}}$ along $x$ axis $\left(\phi=90^{\circ}\right)$. Minimizing the energy with respect to $\theta$ with $\phi=90^{\circ}$ 
gives

$$
\theta=\sin ^{-1}\left(\frac{\mu_{0} M_{\mathrm{s}} H}{2 K_{\mathrm{u}}}\right) .
$$

Thus, the out-of-plane component of $M$ is

$$
M_{\mathrm{z}}=M \sqrt{1-\left(M \mu_{0} H / 2 K_{\mathrm{u}}\right)^{2}} .
$$

Figure 2.20(b) shows the normalized out-of-plane magnetization, $M_{\mathrm{z}}$, as a function of in-plane field. Firstly, an out-of-plane (OP) field $\mu_{0} H_{\mathrm{z}}=9 \mathrm{mT}$ is applied to saturate the magnetization of the trilayer. Then an in-plane (IP) field $\mu_{0} H_{\mathrm{x}}$ is swept along the $x$ axis, which leads to the normalized magnetization rotating from the saturated OP $\left(M_{\mathrm{z}}=1\right)$ to IP state $\left(M_{\mathrm{z}}=0\right)$. The Stoner-Wohlfarth model is used to fit the anisotropy field $\mu_{0} H_{\mathrm{k}}[88,103]$. In the low field regime, -80 to $80 \mathrm{mT}$, the moments rotate coherently following the Stoner-Wohlfarth model, which can be fitted by equation 2.13 (as shown in the red line of Figure 2.20(b)). It should be noted at high fields the magnetization deviates from the single domain Stoner-Wohlfarth behaviour due to the nucleation of magnetic domains [104]. The anisotropy field $\mu_{0} H_{\mathrm{k}}$ is found to be $163 \mathrm{mT}$, which is defined as the point where the extrapolated coherent rotation crosses $M_{\mathrm{z}}=0$. Thus, the effective magnetic anisotropy constant $K_{\text {eff }}=(1 / 2) \mu_{0} H_{\mathrm{k}} M_{\mathrm{s}}$ can be calculated once the $M_{\mathrm{s}}$ is obtained from a SQUID measurement.

\section{Magnetic bubble domain expansion}

MOKE can also be used to verify the existence of DMI-stabilized Néel domain walls in thin films by imaging the magnetic bubble domain expansion driven by a magnetic field pulse [105]. The sequence of measurement is as follows:

- Place the sample under the objective lens of MOKE.

- Saturate the sample along OP direction (up or down) (see Figure 2.21(a)). 

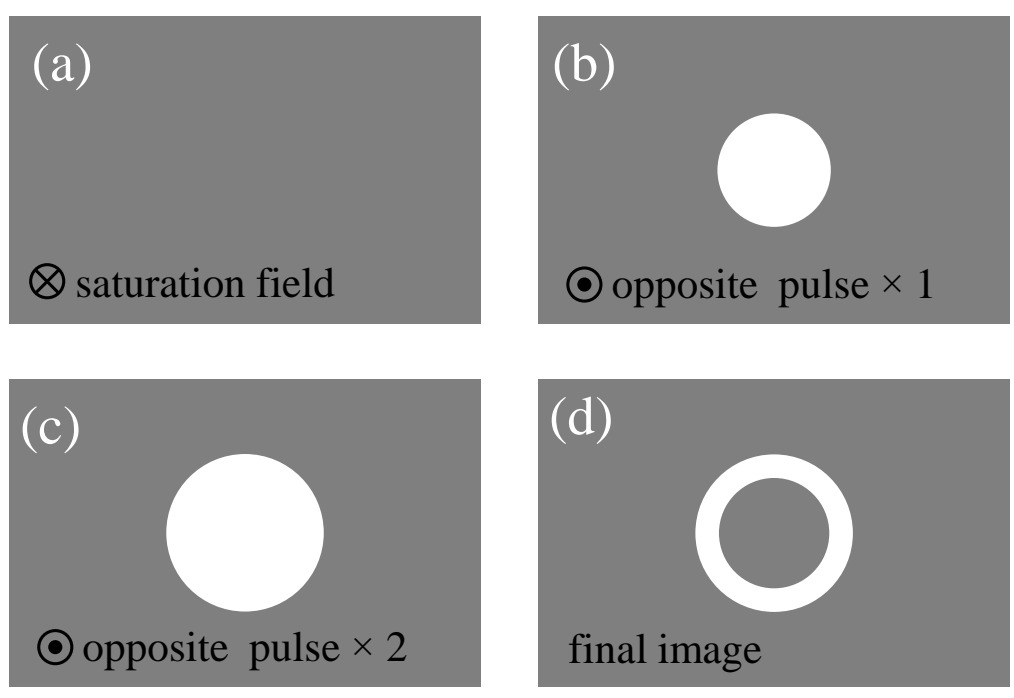

Figure 2.21: Schematic of magnetic bubble expansion. The gray and white colour denote the direction of magnetization along up and down direction, respectively.

- Apply an opposite OP magnetic field pulse to nucleate a small bubble domain (see Figure 2.21(b)). If another pulse is applied, the domain wall will expand as shown in Figure 2.21(c).

- take the image (b) as a background firstly before applying the second pulse. And then apply the second pulse so that the image (d) can be observed.

- Save the final image.

Here the $\mathrm{MgO}(1.6 \mathrm{~nm}) / \mathrm{Mn}_{2} \operatorname{CoAl}(2.6 \mathrm{~nm}) / \mathrm{Pd}(2.2 \mathrm{~nm})$ stack was examined. Figure 2.22(a) shows a typical image of a symmetrically expanding magnetic bubble under a $700 \mathrm{~ms}$ pulse of OP field, $\mu_{0} H_{\mathrm{z}}=9.4 \mathrm{mT}$. By applying the same pulse of OP field under an IP field bias, a clearly asymmetric expansion is observed where the DW moves in the direction of the IP field faster and further than the one moving against the IP field, as shown in Figures 2.22(b)-(c). It is well known that chiral Néel DWs [106, 107] expand asymmetrically under application of an IP field due to the DMI 

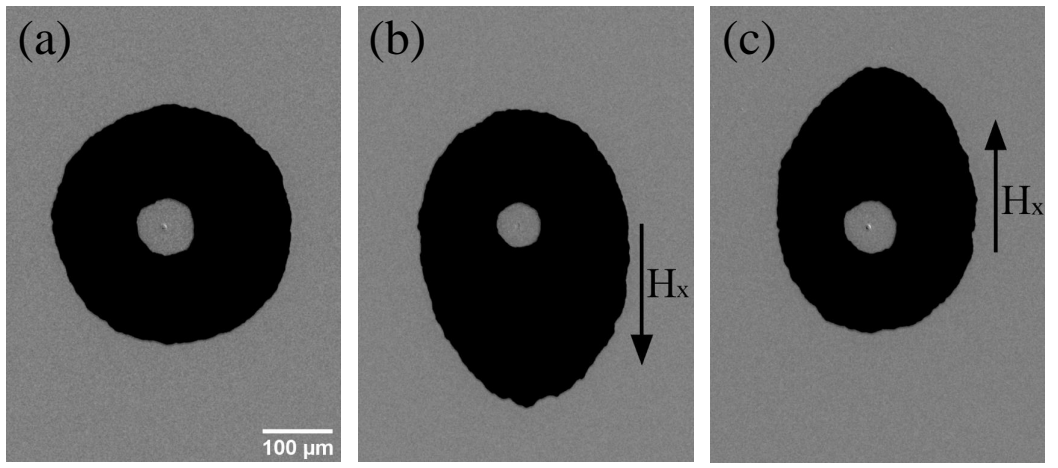

Figure 2.22: (a) DW expansion for stack $\mathrm{MgO}(1.6 \mathrm{~nm}) / \mathrm{Mn}_{2} \mathrm{CoAl}(2.6 \mathrm{~nm}) / \mathrm{Pd}(2.2$ $\mathrm{nm}$ ). DW displacement after applying a pulse of OP field, $\mu_{0} H_{\mathrm{z}}=9.4 \mathrm{mT}$, with $700 \mathrm{~ms}$. (b) DW displacement after applying OP field, $\mu_{0} H_{\mathrm{z}}=9.4 \mathrm{mT}$, with 700 ms under $\mu_{0} H_{\mathrm{x}}=-35.5 \mathrm{mT}$. (c) DW displacement after applying OP field, $\mu_{0} H_{\mathrm{z}}$ $=9.4 \mathrm{mT}$, with $700 \mathrm{~ms}$ under $\mu_{0} H_{\mathrm{x}}=23.7 \mathrm{mT}$.

modifying the DW energy density, whereas a Bloch DW would not expand asymmetrically. This is the evidence that the magnetic domains in $\mathrm{MgO} / \mathrm{Mn}_{2} \mathrm{CoAl} / \mathrm{Pd}$ system have Néel-type chiral DWs.

\section{MOKE image processing}

Here, the software or method used to analyze the MOKE images is briefly summarised. The number and diameter of the skyrmions are analyzed by using the particle counting method in Image [108]. The fractal dimension of domains is calculated by the box-counting method [109] by using the FracLac plugin in ImageJ. The nearest neighbour distance (NND) of skyrmions is calculated using the Nnd plugin in ImageJ. The domain period of demagnetizing image is extracted using a two-dimensional fast Fourier transform by the Gwyddion software [110]. 


\section{Chapter 3}

\section{Thickness dependence of perpendicular magnetic anisotropy in $\mathrm{Mn}_{2} \mathrm{CoAl}$ ultrathin films}

\subsection{Introduction}

In this thesis, most research focuses on $\mathrm{Mn}_{2} \mathrm{CoAl}$ films. The trilayers, $\mathrm{MgO} / \mathrm{Mn}_{2} \mathrm{CoAl} / \mathrm{Pd}$ ultrathin films, exhibit perpendicular magnetic anisotropy (PMA) which has been observed in my group [70]. However, the Pd thickness dependence of PMA and magnetic morphology in this system are still unknown. Here, the magnetization of thick $\mathrm{Mn}_{2} \mathrm{CoAl}$ films (100 nm) was firstly introduced. Then, the magnetization of $\mathrm{MgO} / \mathrm{Mn}_{2} \mathrm{CoAl} / \mathrm{Pd}$ trilayers was investigated. The Pd thickness dependence of magnetization of trilayers was also addressed. Finally, the domain structures in the trilayers with various Pd thickness were investigated. The rest of this thesis is based on the results and understanding obtained in this chapter. 


\subsection{Results}

\subsubsection{Characterization of thick $\mathrm{Mn}_{2} \mathrm{CoAl}$}

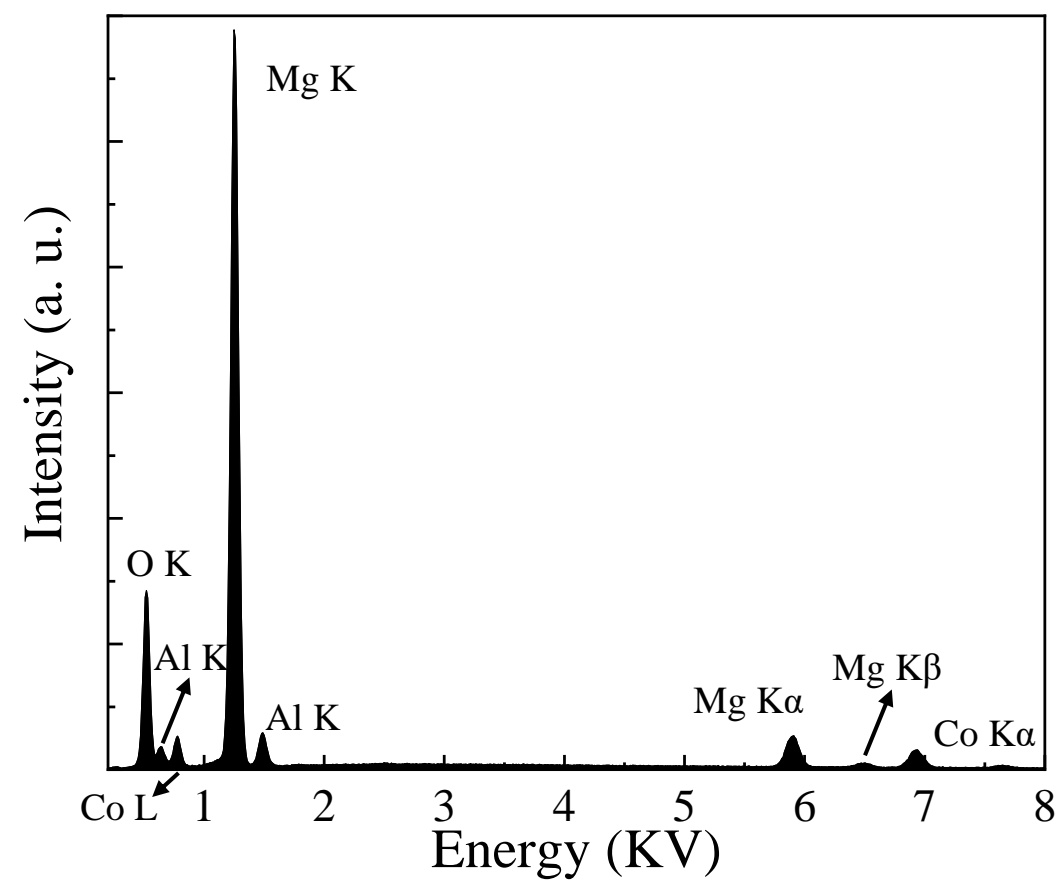

Figure 3.1: An experimental EDX spectrum of $100 \mathrm{~nm} \mathrm{Mn}{ }_{2} \mathrm{CoAl}$ thin film on $\mathrm{MgO}$ substrate measured at $25 \mathrm{KV}$.

Here, the basic properties of thick $\mathrm{Mn}_{2} \mathrm{CoAl}(100 \mathrm{~nm})$ films will be introduced, which have been fully studied by my group [68]. The $\mathrm{Mn}_{2} \mathrm{CoAl}$ thin films are deposited on single-crystalline $\mathrm{MgO}(001)$ substrates by the magnetron sputtering with a base pressure below $3 \times 10^{-8}$ Torr. The $\mathrm{MgO}$ substrates are held for $1 \mathrm{~h}$ at $550{ }^{\circ} \mathrm{C}$, and then the films are deposited on substrates under a $6 \mathrm{mTorr}$ of Ar with a growth rate of $0.83 \AA / \mathrm{s}$. After growth, the films are cooled down to room temperature at a rate of $\sim 10 \mathrm{~K} /$ minute. The elemental composition of $\mathrm{Mn}_{2} \mathrm{CoAl}$ was calibrated by EDX, as shown in Figure 3.1. The stoichiometry is $\mathrm{Mn}_{1.68} \mathrm{Co}_{1.16} \mathrm{Al}_{1.17}$, in which part of Mn has been lost. 
Figure 3.2(a) shows a hysteresis loop of $100 \mathrm{~nm} \mathrm{Mn}_{2} \mathrm{CoAl}$ film measured by SQUID at $300 \mathrm{~K}$. The film has a saturation magnetization of $1.52 \times 10^{5} \mathrm{~A} / \mathrm{m}$ with a coercive field of $13.3 \mathrm{mT}$. The saturation magnetization of this thin film is half of the value of bulk [66] which can be ascribed to the non-stoichiometry and the bad and black surface grown at high temperature. The temperature dependence of magnetization is measured from 3 to $700 \mathrm{~K}$, as shown in Figure 3.2(b), which meets the wellknown Bloch $T^{3 / 2}$ law [111]. It should be noted that the data from 3 to $400 \mathrm{~K}$ and 400 to $700 \mathrm{~K}$ was measured by SQUID and PPMS, respectively. The data is fitted by the empirical relation $M(T)=M(0)\left[1-\left(T / T_{\mathrm{c}}\right)^{2}\right]^{1 / 2}$ (red line). Extrapolating this fit to zero magnetization gives a Curie temperature of approximately $585 \mathrm{~K}$, in agreement with other group's results for $\mathrm{Mn}_{2} \mathrm{CoAl}$ thin films [112] but smaller than the $720 \mathrm{~K}$ of bulk $\mathrm{Mn}_{2} \mathrm{CoAl}$ [66].
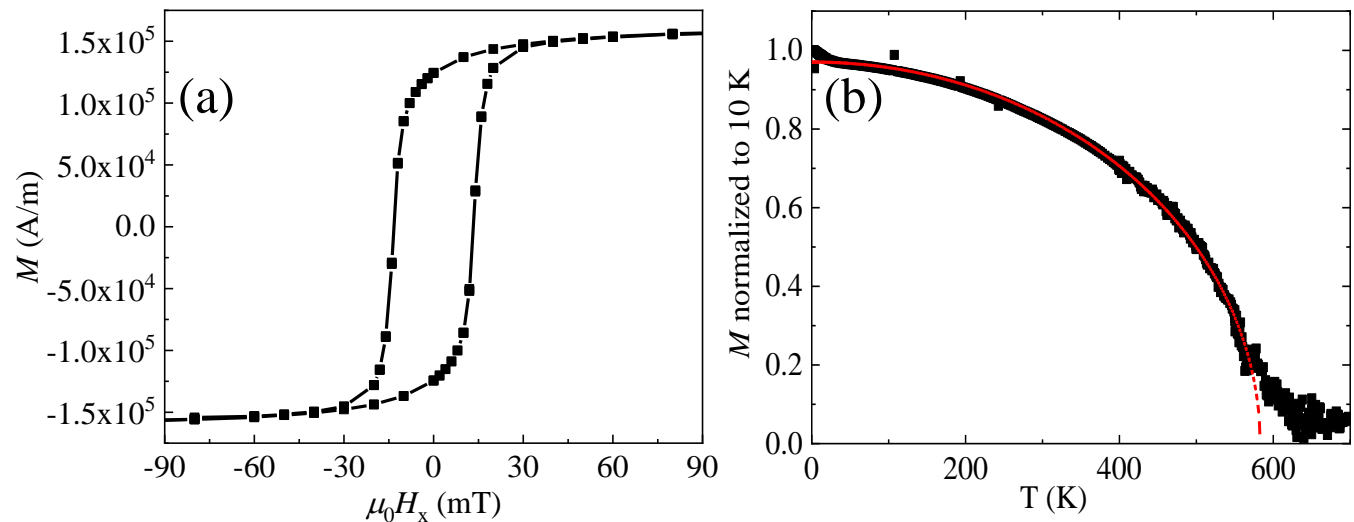

Figure 3.2: (a) Hysteresis loop of $100 \mathrm{~nm} \mathrm{Mn}_{2} \mathrm{CoAl}$ measured with an in-plane magnetic field at $300 \mathrm{~K}$. The magnetic field swept from $90 \mathrm{mT}$ down to $-90 \mathrm{mT}$. (b) Temperature dependence of magnetization under a $100 \mathrm{mT}$ in-plane magnetic field [68]. The red line is an extrapolation to estimate the Curie temperature by $M(T)=M(0)\left[1-\left(T / T_{\mathrm{c}}\right)^{2}\right]^{1 / 2}$. 


\subsubsection{Characterization of trilayers}

(a)

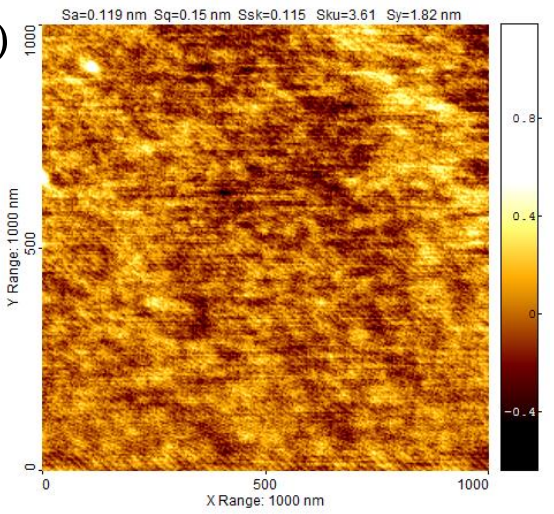

(b)

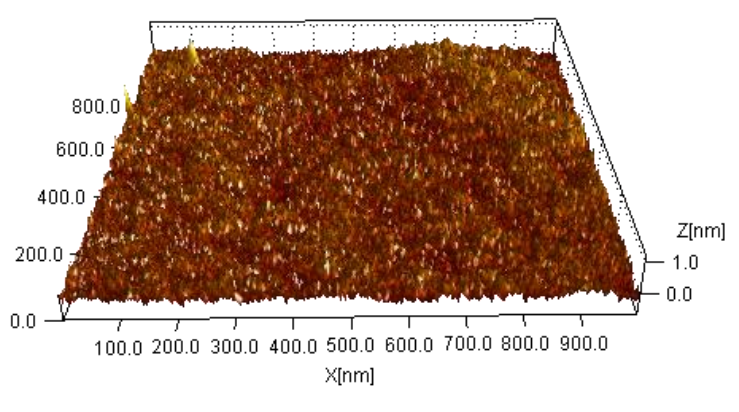

Figure 3.3: The atomic force microscopy image of $\mathrm{MgO} / \mathrm{Mn}_{2} \mathrm{CoAl} / \mathrm{Pd}$ ultrathin film for two dimensions (a) and three dimensions (b) $\left(5 \times 5 \mu \mathrm{m}^{2}\right)$ with colour contrast (dark to bright) for the height scale.

After introducing $\mathrm{Mn}_{2} \mathrm{CoAl}$ thick thin films, the $\mathrm{MgO} / \mathrm{Mn}_{2} \mathrm{CoAl} / \mathrm{Pd}$ ultrathin films are systematically investigated. The details of trilayers' deposition processes present as following. Thermally oxidized Si substrates are exposed to a 5 minutes $\mathrm{Ar}^{+}$plasma cleaning in the growth chamber immediately to remove residual water and molecules before deposition. In this chapter, ultrathin films consisting of $\mathrm{MgO} / \mathrm{Mn}_{2} \mathrm{CoAl} / \mathrm{Pd}$ trilayers are grown at ambient temperature while rotating the sample holder and then post-annealed in situ for $1 \mathrm{~h}$ at $300{ }^{\circ} \mathrm{C}$. $\mathrm{MgO}$ is RF sputtered at $100 \mathrm{~W}$ in 3 mTorr Ar at a growth rate of $0.05 \AA / \mathrm{s}$. $\mathrm{Mn}_{2} \mathrm{CoAl}$ and Pd are DC sputtered at $100 \mathrm{~W}$ in $5 \mathrm{mTorr}$ Ar and $20 \mathrm{~W}, 2 \mathrm{mTorr}$ Ar, respectively, at growth rates of 0.84 and $0.46 \AA / \mathrm{s}$, respectively.

The film roughness was measured by AFM, as shown in Figure 3.3, with a root-mean-square roughness $\left(R_{\mathrm{q}}\right)$ of $0.15 \mathrm{~nm}$ confirming a smooth surface morphology.

Magnetic hysteresis loops of $\mathrm{MgO}(2.0 \mathrm{~nm}) / \mathrm{Mn}_{2} \operatorname{CoAl}(2.6 \mathrm{~nm}) / \mathrm{Pd}(2.0$ $\mathrm{nm})$ are measured by SQUID with the external magnetic field along the OP direction at $300 \mathrm{~K}$, as shown in Figure 3.4. It shows a square loop 
meaning that there is a hysteresis effect along the OP direction. In other words, it exhibits PMA. The coercive field $\mu_{0} H_{\mathrm{c}}$ and saturation magnetization $M_{\mathrm{s}}$ are $0.6 \mathrm{mT}$ and $3.15 \times 10^{5} \mathrm{~A} / \mathrm{m}$, respectively. The value of $M_{\mathrm{s}}$ in the $\mathrm{Mn}_{2} \mathrm{CoAl}$ trilayer is almost same as bulk and thick $\mathrm{Mn}_{2} \mathrm{CoAl}$ thin films $[66,113]$ meaning that there is no obvious dead layer.

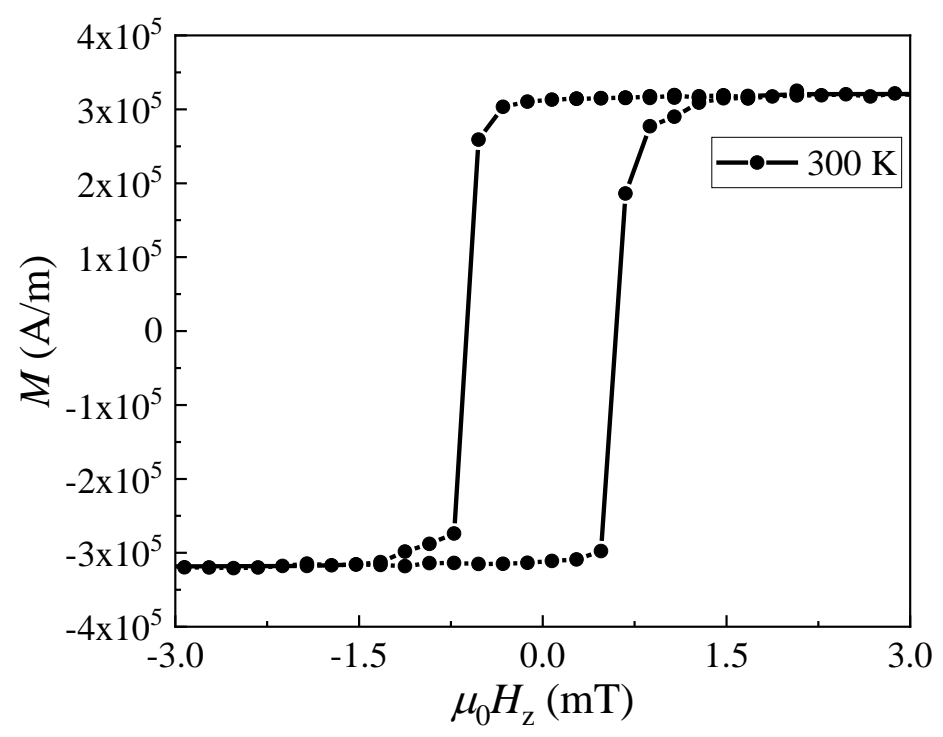

Figure 3.4: Hysteresis loop of $\mathrm{MgO}(2.0 \mathrm{~nm}) / \mathrm{Mn}_{2} \operatorname{CoAl}(2.6 \mathrm{~nm}) / \mathrm{Pd}(2.0 \mathrm{~nm})$ measured at $300 \mathrm{~K}$.

\subsubsection{Thickness dependence of Pd}

It is known that the PMA relates to the nonmagnetic heavy metal layer due to spin-orbit interactions [39, 114]. Here, trilayers with different thicknesses of Pd to modify the PMA of $\mathrm{Mn}_{2} \mathrm{CoAl}$ trilayers were grown. Figure 3.5 shows the hysteresis loops measured by MOKE with OP field for various thickness of $\mathrm{Pd}$ in the trilayers, $t_{\mathrm{Pd}}$ from 2.2 to $4.4 \mathrm{~nm}$. For trilayers with $\mathrm{Pd}(2.2-3.6 \mathrm{~nm})$, they show square loops. However, once the $t_{\mathrm{Pd}}$ increases above $3.6 \mathrm{~nm}$, the magnetic transition is not sharp, and the value of magnetization at $0 \mathrm{mT}$ does not equal to the saturation mag- 


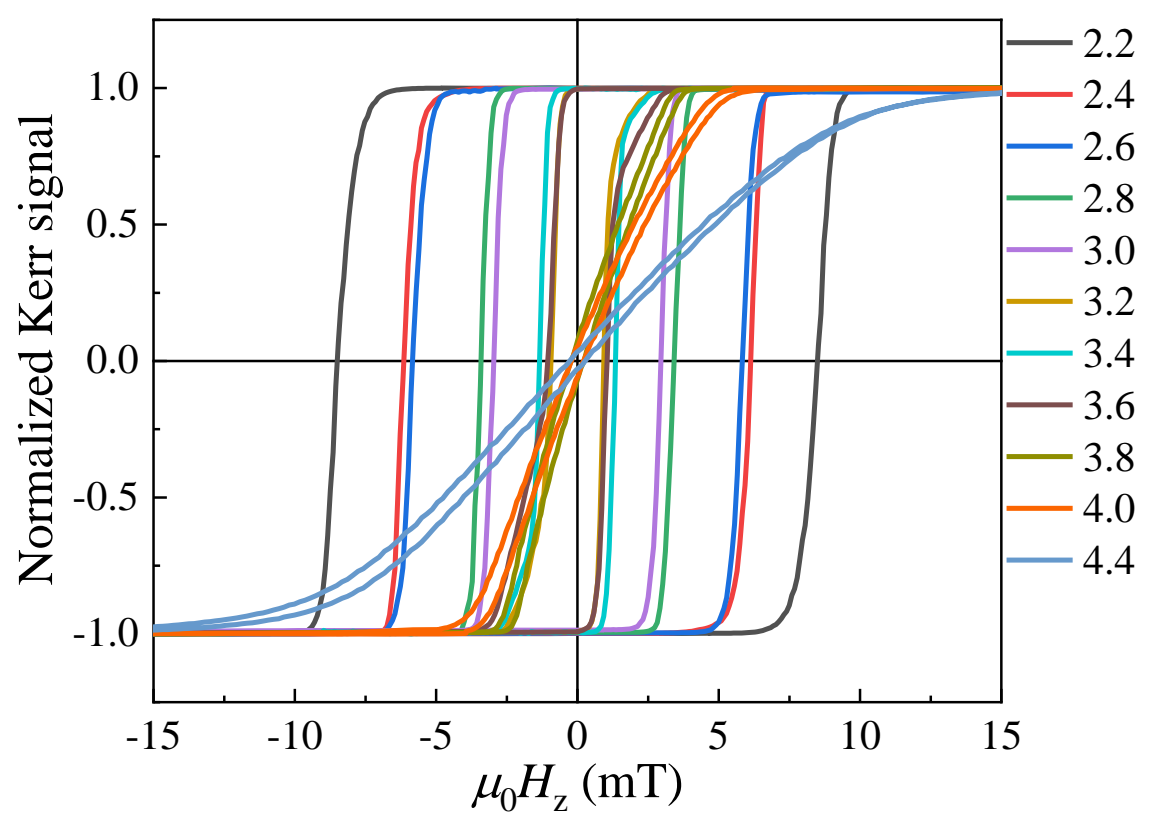

Figure 3.5: Hysteresis loops of $\mathrm{MgO}(1.6 \mathrm{~nm}) / \mathrm{Mn}_{2} \mathrm{CoAl}(2.6 \mathrm{~nm}) / \mathrm{Pd}(2.2-4.4 \mathrm{~nm})$ measured at ambient temperature with a field along the OP direction.

netization anymore suggesting that samples is at the spin-reorientation transition where magnetization rotates from the OP and IP direction. Figure 3.6 shows the important parameters, $M_{\mathrm{r}} / M_{\mathrm{s}}, \mu_{0} H_{\mathrm{c}}$ and $K_{\text {eff }}$, as function of $t_{\mathrm{Pd}}$. The $M_{\mathrm{r}} / M_{\mathrm{s}}$ is 1 for trilayers with $\mathrm{Pd}(2.2-3.6 \mathrm{~nm})$ indicating that the remanent magnetization keeps the same direction as that of saturation magnetization along OP direction, but it suddenly drops to close to 0 . The $K_{\text {eff }}$ calculation has been introduced in the section 2.5.3. Both $\mu_{0} H_{\mathrm{c}}$ and $K_{\text {eff }}$ decrease as increasing the thickness of Pd, as shown in Figures 3.6(b) and (c), indicating that the magnetic anisotropy of trilayers with thick Pd layer becomes weak along OP direction and transfers to IP direction. It should be noted that the $K_{\text {eff }}$ is still positive suggesting that the samples still have PMA.

To further understand the effects of the variation of Pd thickness, Magnetic domains are captured by polar MOKE microscopy at ambient temperature. Figure 3.7 shows the typical magnetic domain images for thin 
films with various Pd thickness at the transition regime, closing coercive field, where the direction of magnetization flips to the opposite direction. These images are captured after sweeping an OP field from positive magnetic saturation (starting field, $1.5 \mathrm{mT}$ ) to the terminal field noted in the Figure. For trilayer with $2.2 \mathrm{~nm} \mathrm{Pd}$, a big uniform domain can be observed, as shown in Figure 3.7(a). By increasing the Pd thickness to $2.8 \mathrm{~nm}$, the size of uniform domain decreases, and part of it collapse into labyrinth domains, as shown in Figure 3.7(b). By continuing to increase the Pd thickness to $3.2 \mathrm{~nm}$, the labyrinth domains dominate. It should be noted that the scale bars in Figures 3.7(a) and (c) correspond to different lengths. Interestingly, when the $t_{\mathrm{Pd}}=3.8 \mathrm{~nm}$, skyrmions with average diameter $\sim 1 \mu \mathrm{m}$ can be observed, which will be discussed in the next two chapters. From the hysteresis loop of this sample (see Figure 3.5), the magnetic anisotropy of trilayer is close to the transition of IP direction.
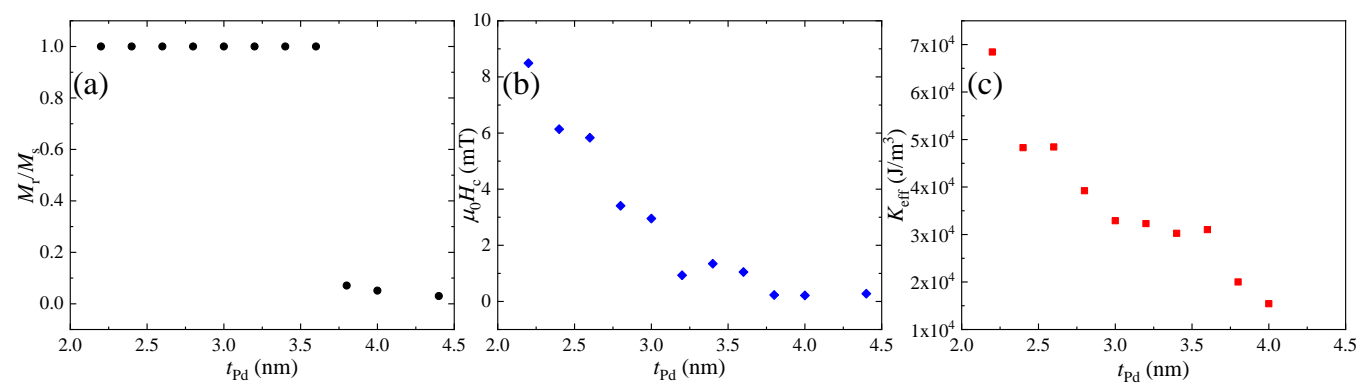

Figure 3.6: $M_{\mathrm{r}} / M_{\mathrm{s}}, \mu_{0} H_{\mathrm{c}}$ and $K_{\mathrm{eff}}$ as function of $t_{\mathrm{Pd}}$ in $\mathrm{MgO} / \mathrm{Mn}_{2} \mathrm{CoAl} / \mathrm{Pd}$ stacks.

\subsection{Discussion and conclusions}

By systematically studying the magnetic properties of $\mathrm{MgO} / \mathrm{Mn}_{2} \mathrm{CoAl} / \mathrm{Pd}$ trilayers influenced by the thickness of Pd layer, these samples can be prepared for different purposes. For example, trilayers with thick Pd show skyrmions which can be used to investigate the magnetic behaviours of 

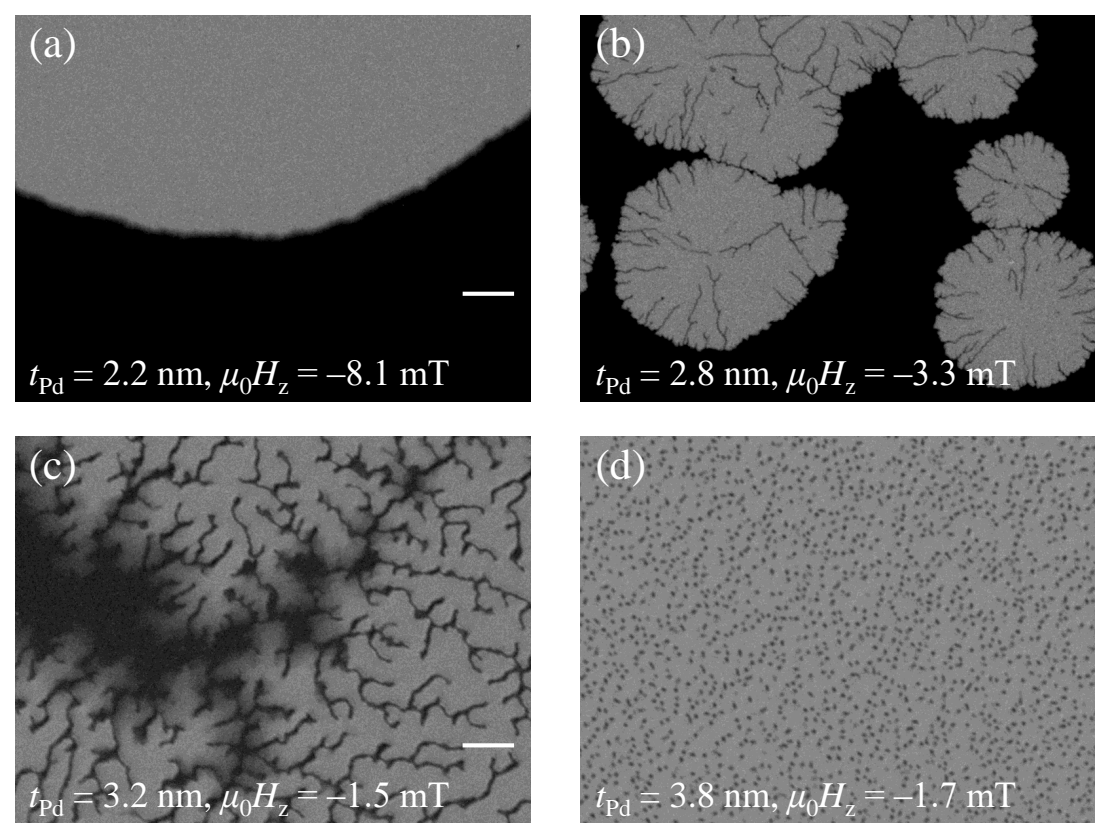

Figure 3.7: Typical magnetic domain images for various $\mathrm{MgO} / \mathrm{Mn}_{2} \mathrm{CoAl} / \mathrm{Pd}$ trilayers at certain magnetic field. $t_{\mathrm{Pd}}$ denotes the thickness of Pd layer. The scale bar corresponds to $80 \mu \mathrm{m}$ in (a) and (b). The scale bar corresponds to $8 \mu \mathrm{m}$ in (c) and $(\mathrm{d})$.

skyrmion-based devices. Further, to easily generate skyrmions by external stimulation, like magnetic field, temperature, and voltage, a trilayer can be designed to exhibit labyrinth domain which closes to the skyrmion regime.

In summary, the magnetization, anisotropy and magnetic morphology of $\mathrm{MgO} / \mathrm{Mn}_{2} \mathrm{CoAl} / \mathrm{Pd}(t \mathrm{~nm})$ trilayers were investigated. By carefully optimising the growth condition and thickness of each layer, the PMA has been achieved in $\mathrm{Mn}_{2} \mathrm{CoAl}$-based trilayers. By fine tuning the $t_{\mathrm{Pd}}$, the effective magnetic anisotropy can be decreased by increasing the Pd thickness. Finally, skyrmions can be observed for the sample with a magnetic anisotropy at spin-reorientation transition regime. 


\section{Chapter 4}

\section{Skyrmions generated by in-plane magnetic field in $\mathrm{Mn}_{2} \mathrm{CoAl}$ ultrathin films}

\subsection{Introduction}

For ferromagnet/heavy metal ultrathin film bilayers or multilayers with PMA, skyrmions can be observed due to the competition among the exchange interaction, magnetic anisotropy interaction and DMI [7]. In order to generate skyrmions, one has to tune these interactions. Since the strength of the exchange interaction is hard to change in a fixed system, researchers are focusing on modulating the DMI and the interfacial magnetic anisotropy in multilayers. However, both are very sensitive to the layer thickness and interface quality $[115,116,117]$ so it can require an extensive experimental campaign to produce multiple samples attempting to find that narrow range. This is a challenge for sample preparation and is curtailing possible applications. To truly speed up the work to optimise skyrmion conditions it would be most useful to develop a method to adjust the relevant magnetic parameters that produce skyrmions after a sample has been produced. 
On the other hand, it is also well known that skyrmions can be formed from labyrinth domains or uniformly ferromagnetic domains by varying temperature, magnetic field and current in thin films [8, 50, 118]. An approach which can separate these formations of skyrmions is still lacking.

Fractal analysis, a method that allows the complexity of patterns to be quantified, can be a useful tool to study and distinguish these domains [119]. A fractal is a shape made of parts with irregular and self-similar features at all scales [120]. The fractal dimension, $D_{\mathrm{f}}$, is a measure of the degree of self-similarity of a pattern, and thus can be used as a classifier. In magnetic materials, $D_{\mathrm{f}}$ has been used to describe and distinguish the multi-branched domains formed in garnet bubble films at various external magnetic fields [119]. In FePt/Pt(001) and $\mathrm{FePt} / \mathrm{MgO}(001)$ thin films with PMA, Attané et al., compared the fractal dimension measured during the magnetization reversal process to conclude that the structures of the magnetic domains for both are similar at large scales, but show very different domain wall geometries at small scale [121]. Thus, fractal analysis could be a powerful method to describe and distinguish the evolution of skyrmions formed from other magnetic domains.

In this chapter, the formation and evolution of skyrmions were investigated in $\mathrm{MgO} / \mathrm{Mn}_{2} \mathrm{CoAl} / \mathrm{Pd}$ ultrathin films with PMA by gradually modifying the effective magnetic anisotropy using an in-plane magnetic field bias. Also, the fractal dimension was demonstrated as a tool to describe and distinguish the evolution of magnetic domains and skyrmions.

\subsection{Results}

\subsubsection{Magnetization and chiral domain wall}

The hysteresis loop of a $\mathrm{MgO}(1.6 \mathrm{~nm}) / \mathrm{Mn}_{2} \operatorname{CoAl}(2.6 \mathrm{~nm}) / \mathrm{Pd}(3.2 \mathrm{~nm}) \mathrm{ul}-$ trathin film is measured with the external magnetic field along the OP direction at ambient temperature $(\sim 294 \mathrm{~K})$ by polar MOKE, as shown in 
Figure 4.1(a). This sample shows clear PMA with a coercive field of $\mu_{0} H_{\mathrm{c}}$ $=0.9 \mathrm{mT}$. Figure 4.1(b) shows the normalized MOKE signal as a function of in-plane (IP) field which can identify the effective magnetic anisotropy (see section 2.5.3). To determine the magnetic anisotropy, first an OP field $\mu_{0} H_{\mathrm{z}}=3.3 \mathrm{mT}$ is applied to saturate the magnetization of the multilayer, then an IP field $\mu_{0} H_{\mathrm{x}}$ is swept along the $x$ axis from 0 to $300 \mathrm{mT}$ [103]. The black dot curve of Figure 4.1(b) represents the normalized magnetization rotated from the saturated out-of-plane state $\left(m_{\mathrm{z}}=1\right)$ to in-plane (IP) $\left(m_{\mathrm{z}}=0\right)$. Assuming there is no magnetization component along the $y$ axis and the normalized saturation magnetization $m=1$, one can obtain $m_{\mathrm{x}}=\sqrt{\left(1-m_{\mathrm{z}}^{2}\right)}$. In the low field regime, 0 to $50 \mathrm{mT}$, the moments rotate coherently following the Stoner-Wohlfarth model, which can be fitted by equation $m_{\mathrm{z}}=m \sqrt{1-\left(m \mu_{0} H / 2 K_{\mathrm{u}}\right)^{2}}$ [102] (as shown in the red dash line of Figure 4.1(b)). The anisotropy field $\mu_{0} H_{\mathrm{k}}$ is found to be $205 \mathrm{mT}$, which is defined as the point where the extrapolated coherent rotation crosses $m_{\mathrm{z}}=0$. Thus, the effective magnetic anisotropy constant $K_{\text {eff }}=(1 / 2) \mu_{0} H_{\mathrm{k}} M_{\mathrm{s}}$ is $3.23 \times 10^{4} \mathrm{~J} / \mathrm{m}^{3}$, where $M_{\mathrm{s}}=3.15 \times 10^{5} \mathrm{~A} / \mathrm{m}$ is the saturation magnetization obtained from a SQUID measurement at $300 \mathrm{~K}$, as shown in previous chapter (Figure 3.4).

In order to verify the Néel-type chiral DWs stabilized by DMI at the interface between $\mathrm{Mn}_{2} \mathrm{CoAl}$ and $\mathrm{Pd}$ layer in this system, the magnetic bubble domain expansion driven by a magnetic field pulse is studied using polar MOKE, which has been introduced at section 2.5.3. Here, the $\mathrm{MgO}(1.6 \mathrm{~nm}) / \mathrm{Mn}_{2} \mathrm{CoAl}(2.6 \mathrm{~nm}) / \mathrm{Pd}(2.2 \mathrm{~nm})$ stack was examined. Figures 4.1 (c) shows a typical image of a symmetrically expanding magnetic bubble under a $700 \mathrm{~ms}$ pulse of OP field, $\mu_{0} H_{z}=9.4 \mathrm{mT}$. By applying the same pulse of OP field under an IP field bias, a clearly asymmetric expansion is observed where the DW moves in the direction of the IP field faster and further than the one moving against the IP field, as shown in Figures 4.1(d)-(e). It is well known that chiral Néel DWs [106, 107] expand asymmetrically under application of an IP field due to the DMI modifying 


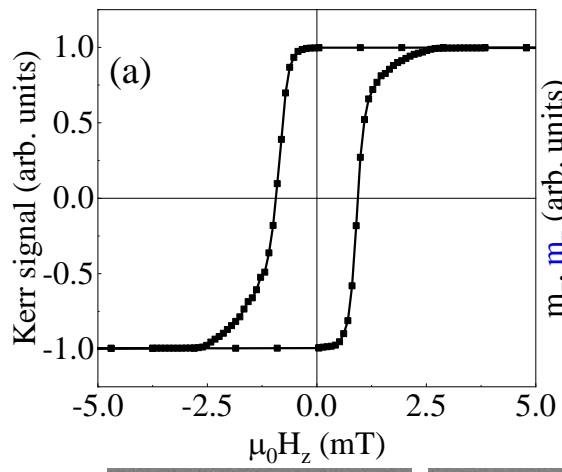

(c)

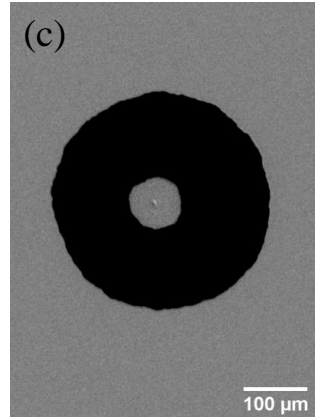

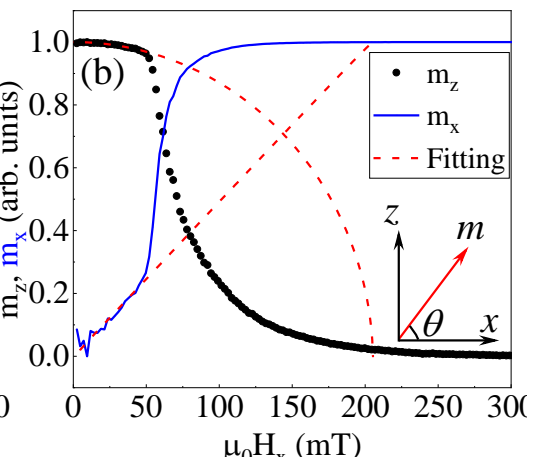

$\mu_{0} \mathrm{H}_{\mathrm{x}}(\mathrm{mT})$
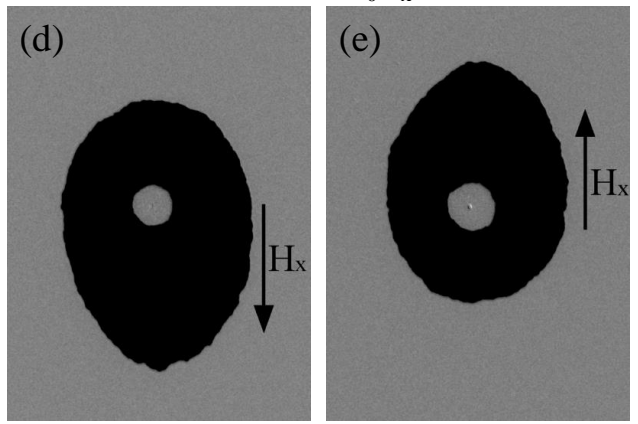

Figure 4.1: (a) Hysteresis loop of $\mathrm{MgO}(1.6 \mathrm{~nm}) / \mathrm{Mn}_{2} \operatorname{CoAl}(2.6 \mathrm{~nm}) / \operatorname{Pd}(3.2 \mathrm{~nm})$ ultrathin film under an OP field at ambient temperature, $\sim 294$ K. (b) Rotation of magnetization from OP to IP direction by sweeping the IP field.(c) DW expansion for stack $\mathrm{MgO}(1.6 \mathrm{~nm}) / \mathrm{Mn}_{2} \operatorname{CoAl}(2.6 \mathrm{~nm}) / \mathrm{Pd}(2.2 \mathrm{~nm})$. DW displacement after applying a pulse of OP field, $\mu_{0} H_{\mathrm{z}}=9.4 \mathrm{mT}$, with $700 \mathrm{~ms}$. (d) DW displacement after applying OP field, $\mu_{0} H_{\mathrm{z}}=9.4 \mathrm{mT}$, with $700 \mathrm{~ms}$ under $\mu_{0} H_{\mathrm{x}}=-35.5 \mathrm{mT}$. (e) DW displacement after applying OP field, $\mu_{0} H_{\mathrm{z}}=9.4 \mathrm{mT}$, with $700 \mathrm{~ms}$ under $\mu_{0} H_{\mathrm{x}}=23.7 \mathrm{mT}$.

the DW energy density, whereas a Bloch DW would not expand asymmetrically. This is evidence that the magnetic domains in this system have Néel-type chiral DWs.

\subsubsection{Skyrmion generation assisted by an in-plane field}

Figure 4.2 shows MOKE imaging of the magnetic domains of the ultrathin film at ambient temperature in various combinations of applied OP 

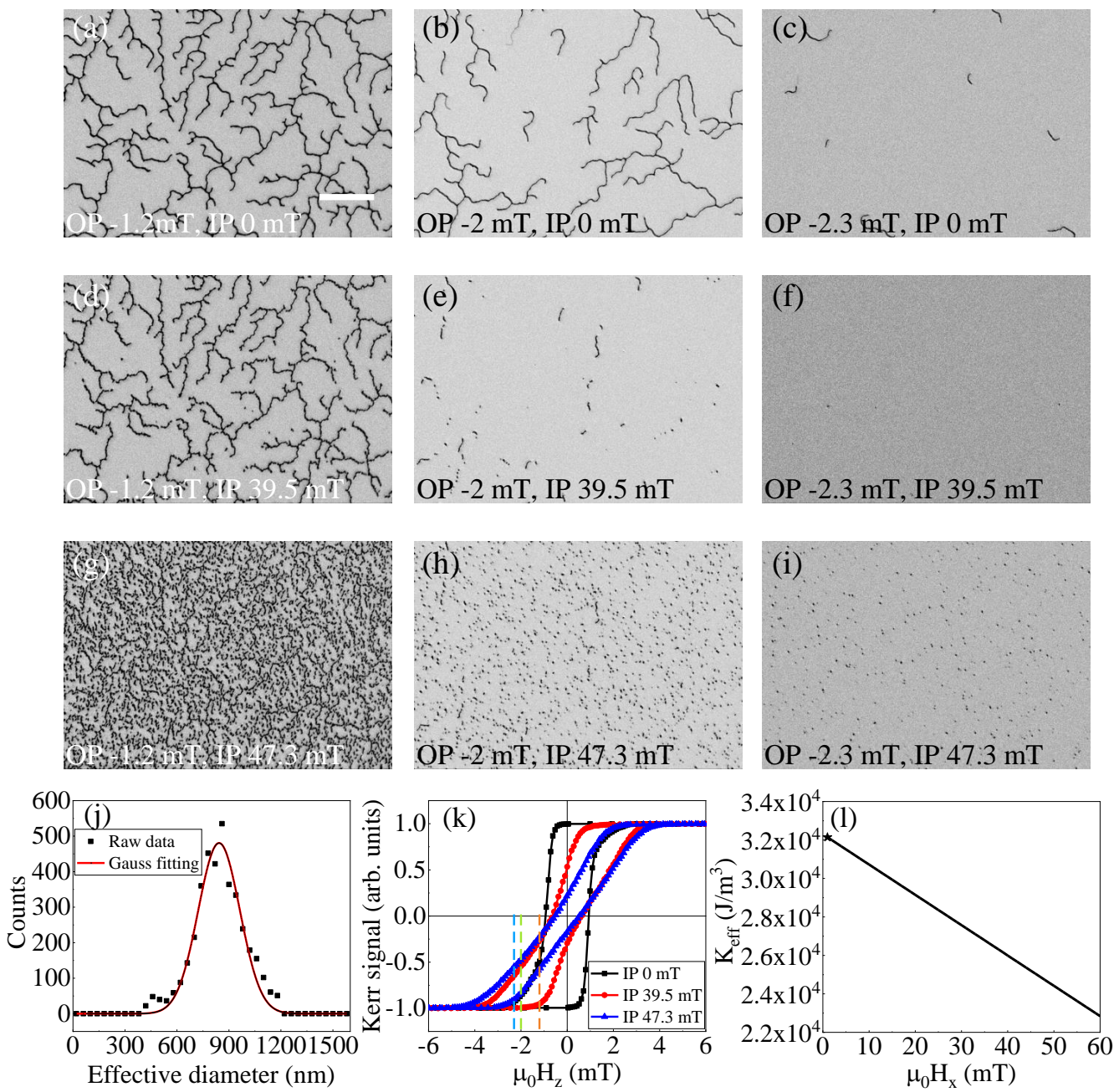

Figure 4.2: (a)-(i) Field-dependent domain morphology of $\mathrm{MgO}(1.6 \mathrm{~nm})$ $/ \mathrm{Mn}_{2} \mathrm{CoAl}(2.6 \mathrm{~nm}) / \mathrm{Pd}(3.2 \mathrm{~nm})$ at ambient temperature. The black and grey features represent magnetization $+m_{\mathrm{z}}$ and $-m_{\mathrm{z}}$, respectively. The scale bar in (a) corresponds to $25 \mu \mathrm{m}$. (j) Counts of skyrmions as a function of effective diameter extracted from (h). The solid line is a fit with a Gaussian distribution. (k) Magnetic hysteresis loops under an IP field 0, 39.5 and $47.3 \mathrm{mT}$, respectively, by sweeping the OP field. The dash lines represent $\mu_{0} H_{\mathrm{z}}=-1.2,-2$ and $-2.3 \mathrm{mT}$, respectively. (1) The effective magnetic anisotropy constant $K_{\text {eff }}$ as a function of IP field. The star represents experimental value extracted from Figure 4.1(b). 
and IP external magnetic fields. Figures 4.2(a)-(c) are each captured after sweeping an OP field. Labyrinth domains were observed for terminal fields ranging from -0.9 to $-2.9 \mathrm{mT}$. With an increasing negative terminal field, more labyrinth domains are annihilated until the film magnetization is saturated and the image shows a uniformly ferromagnetic state, with all magnetic moments pointing in the same (negative $z$ ) direction.

Next, the OP field sweeps were repeated with the same terminal fields, but this time, after reaching the terminal field, an IP field was applied as shown in Figures 4.2(d)-(i). Before each image the sample was reset to the saturated state with an OP field of $9.5 \mathrm{mT}$. For the case of $\mu_{0} H_{\mathrm{z}}=$ $-1.2 \mathrm{mT}$ with $\mu_{0} H_{\mathrm{x}}=39.5$ and $47.3 \mathrm{mT}$, finer features could be observed in the labyrinth domains, and skyrmions (which are visualized the black dots in the images) could be generated (Figures 4.2(d) and (g)). When increasing the IP field after reaching a terminal OP field $\mu_{0} H_{\mathrm{z}}=-2 \mathrm{mT}$ (Figure 4.2(b)), most of the labyrinth domains disappear (Figure 4.2(e)), and isolated skyrmions form both from collapsing labyrinth domains as well as nucleating from the featureless regions (Figure 4.2(h)).

In multilayer thin films, both labyrinth and uniformly ferromagnetic domains are typical sources of skyrmions [118, 122]. When starting from a terminal OP field of $\mu_{0} H_{z}=-2.3 \mathrm{mT}$ (Figure 4.2(c)), the remaining labyrinth domains disappear at $\mu_{0} H_{\mathrm{x}}=39.5 \mathrm{mT}$, and the magnetization of the sample is uniform (Figure 4.2(f)). By increasing $\mu_{0} H_{\mathrm{x}}$ to $47.3 \mathrm{mT}$, isolated skyrmions are generated directly from the featureless regions (Figure 4.2(i)).

The distribution of the effective diameter was quantitatively studied, $d_{s}$, from the images showing skyrmions. $d_{s}$ is defined as $d_{s}=2 \sqrt{A / \pi}$, corresponding to a conversion of the distorted skyrmion geometry into a circular shape, where $A$ is the area of the skyrmion. The $d_{s}$ of skyrmions is analyzed by using the particle counting method in ImageJ [108]. By calculating $d_{s}$ of all skyrmions from Figure 4.2(h), an approximately Gaussian distribution of $d_{s}$ centred around 795 $\pm 120 \mathrm{~nm}$ (Figure 4.2(j)) can be obtained. This value is typical for all experiments. Typical skyrmion sizes 
reported in the literature range between $4 \mathrm{~nm}$ and $4200 \mathrm{~nm}$ in various multilayer thin film systems [47].

Using the asymmetric domain wall expansion method for a similar trilayer sample, the formation of Néel-type chiral domain walls(see Figure 4.1) have confirmed. Therefore, the magnetization configuration of the skyrmions is expected to be Néel-type, similar to other PMA trilayers in the literature [47].

Figure 4.2(k) shows hysteresis loops measured by sweeping the OP field under a fixed IP field. For an increased fixed IP field, a higher OP field is required to saturate the thin film in the OP direction. In Figure 4.2(k), the points where the dashed lines (fixed OP field values) intersect with the field loops correspond to the field conditions for the MOKE images of Figures 4.2(a)-(i). Since the $K_{\text {eff }}$ can be written as $(1 / 2) \mu_{0}\left(H_{\mathrm{k}}-H_{\mathrm{x}}\right) M_{\mathrm{s}}$ under a IP field, the IP field gradually decreases the $K_{\text {eff }}$, as shown by the calculation in Figure 4.2(1).

Next is to understand the processes of generation of these skyrmions determined by the IP field. Figure 4.3 shows in finer detail how skyrmions are generated by an increasing IP field with a fixed OP field, $\mu_{0} H_{\mathrm{z}}=-2$ $\mathrm{mT}$, between Figures 4.2(b) and (h). By increasing $\mu_{0} H_{\mathrm{x}}$, labyrinth domains gradually collapse into skyrmions, as shown in the purple region of Figures 4.3(a)-(c). This transformation has been demonstrated before by applying current to cause chiral labyrinth domains in a PMA film to collapse as they squeeze through a constriction [8]. As $\mu_{0} H_{\mathrm{x}}$ is further increased new skyrmions are originated from the uniformly ferromagnetic region, as shown in the orange regions of Figure 4.3(d). Both skyrmions that emerged from the labyrinth domains and the uniform ferromagnetic region disappear after removing the in-plane field when the effective magnetic anisotropy returns to the pristine state. 

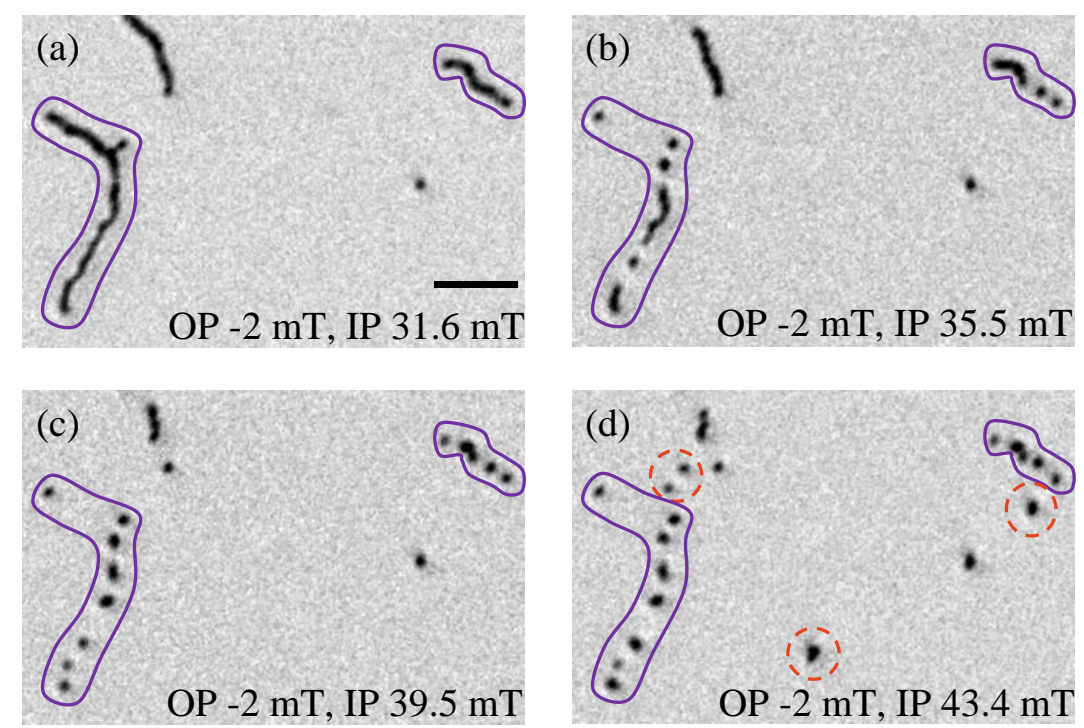

Figure 4.3: (a)-(d) The enlarged domain morphology of $\mathrm{MgO}(1.6 \mathrm{~nm})$ $/ \mathrm{Mn}_{2} \operatorname{CoAl}(2.6 \mathrm{~nm}) / \mathrm{Pd}(3.2 \mathrm{~nm})$ under a combination of OP and IP field at ambient temperature. Skyrmions in the purple (resp. orange) region are formed from labyrinth domains (resp. uniformly saturated magnetic domains). The scale bar in (a) corresponds to $5 \mu \mathrm{m}$.

\subsubsection{DMI calculation}

Here, two methods were used to calculate the DMI coefficient. The exchange stiffness is given by [123]

$$
A_{\mathrm{ex}}=\frac{D_{\mathrm{s}} M_{\mathrm{s}}}{2 g \mu_{\mathrm{B}}}
$$

where $D_{\mathrm{s}}$ is the spin-wave stiffness, $g$ is Landé $g$-factor and $\mu_{\mathrm{B}}$ is Bohr magneton. the $A_{\mathrm{ex}}$ was estimated as $8.99 \mathrm{pJ} / \mathrm{m}$ with $M_{\mathrm{s}}=3.15 \times 10^{5} \mathrm{Am}^{-1}$ (from Figure 3.4), $g=2.05, \mu_{\mathrm{B}}=9.274 \times 10^{-24} \mathrm{~J} / \mathrm{T}, D_{\mathrm{s}}=677 \mathrm{meV} \mathrm{nm}^{2}$ [124]. The DMI can be extracted from the equation [125]

$$
\sigma_{\mathrm{DW}}=4 \sqrt{A_{\mathrm{ex}} K_{\mathrm{eff}}}-\pi D,
$$

where $\sigma_{\mathrm{DW}}$ is the domain wall energy. For ultrathin films with perpendicular magnetic anisotropy, when the thickness of ferromagnetic layer, $t$, is 
much smaller than the domain width, $\sigma_{\text {DW }}$ can be written as [126]

$$
\sigma_{\mathrm{DW}}=\frac{\mu_{0} M_{\mathrm{s}}^{2} t}{\pi} \ln \frac{d}{0.955},
$$

where $d$ is the width of domain period extracted using a two-dimensional fast Fourier transform from the demagnetized domain image, as shown in Figure 4.4. Thus, $D$ can be calculated as $0.44 \mathrm{~mJ} / \mathrm{m}^{2}$ with $t=2.6 \mathrm{~nm}$ and $d$ $=4500 \mathrm{~nm}$.

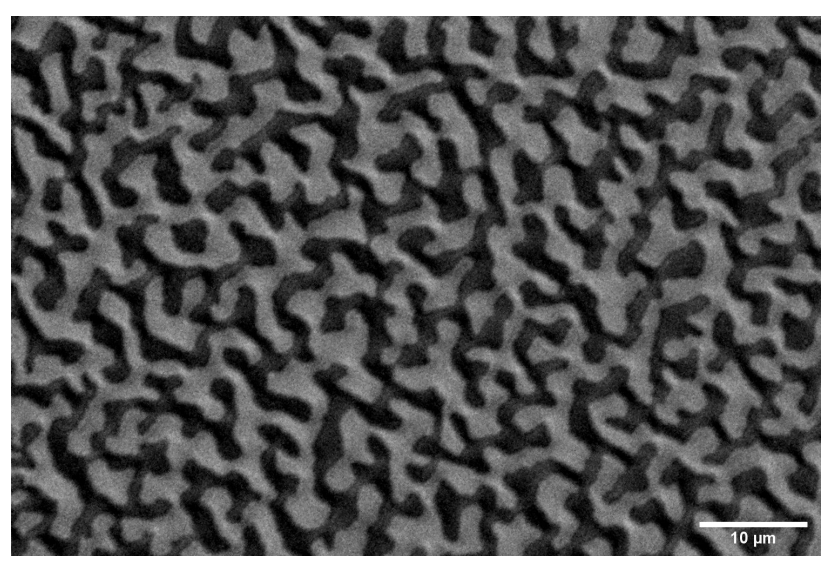

Figure 4.4: Typical image of demagnetized magnetic domain.

There is another method to calculate the DMI. $\sigma_{D W}$ can also be written as [127]

$$
\frac{2 \pi \sigma_{\mathrm{DW}}}{\mu_{0} M_{\mathrm{s}}^{2} t}=\ln \left[1+\left(\frac{d_{\downarrow, \text { min }}}{t}\right)^{2}\right]+\left(\frac{d_{\downarrow, \text { min }}}{t}\right)^{2} \ln \left[1+\left(\frac{d_{\downarrow, \text { min }}}{t}\right)^{-2}\right],
$$

where $d_{\downarrow, \text { min }}$ is the minimal value of downward domain. $d_{\downarrow, \min }$ can be extracted from the domain image under an OP field after processing demagnetization. Figure 4.5(a) shows a typical image under $\mu_{0} H_{\mathrm{z}}=-0.7 \mathrm{mT}$ after demagnetizing. By fitting the domain width by the function $y=$ $a \tanh (b x+c)+a_{0}$, which can be used to describe a hysteresis loop or magnetic saturation curve [128], as shown in Figure $4.5(\mathrm{~b}), d_{\downarrow, \min }$ can be extracted as $810 \mathrm{~nm}$. Thus, $D$ can be calculated as $0.48 \mathrm{~mJ} / \mathrm{m}^{2}$ which agrees 
very well with the value from first method. Thus, the $D=0.46 \mathrm{~mJ} / \mathrm{m}^{2}$ represents the mean of both values.
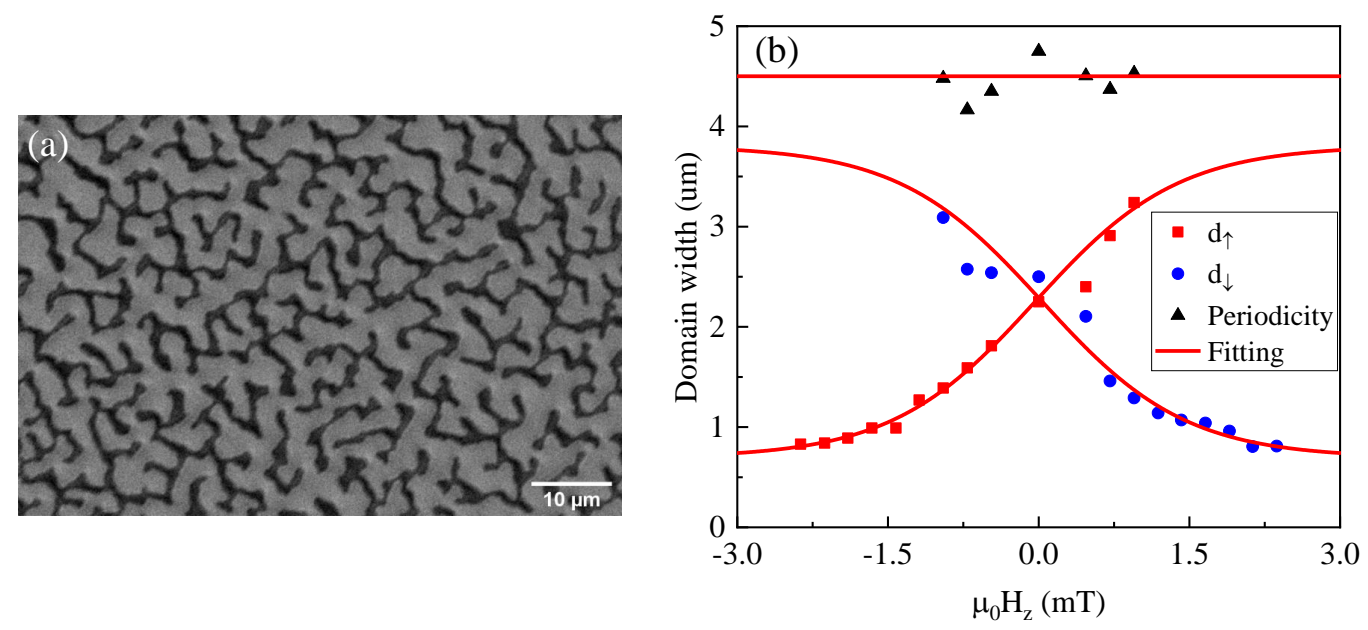

Figure 4.5: (a) Typical image of magnetic domain under $\mu_{0} H_{\mathrm{z}}=-0.7 \mathrm{mT}$ after demagnetizing. Black areas represent the downward domains $d_{\downarrow}$. (b) Domain width as a function of OP field and corresponding fitting by the function $y=$ $a \tanh (b x+c)+a_{0}$.

\subsubsection{Fractal analysis and magnetic phase diagram}

Given that magnetic domains and skyrmions show different levels of complexity and fragmented structures, fractal analysis can be a useful tool to study and distinguish these domains, as mentioned in section 1.6.1.

The fractal dimension $D_{\mathrm{f}}$ of the domain image is extracted by using the box-counting method, a method of gathering data for analyzing complex patterns by breaking a dataset, object, image, etc. into smaller and smaller pieces, typically "box"-shaped, and analyzing the pieces at each smaller scale. [129]. Figure 4.6 shows the processes of fractal analysis [130]. Figure 4.6(a) is the original MOKE microscope image. Before doing fractal analysis, a binary contour pattern of magnetic domains needs to be identified, as shown in Figure 4.6(b). Then, the squares were counted with 
different size needed to cover the contour pattern. Figures 4.6(c) and (d) show there are 956 (resp. 333) squares needed with size 8 (resp. 20) pixels. $D_{\mathrm{f}}$ is defined as
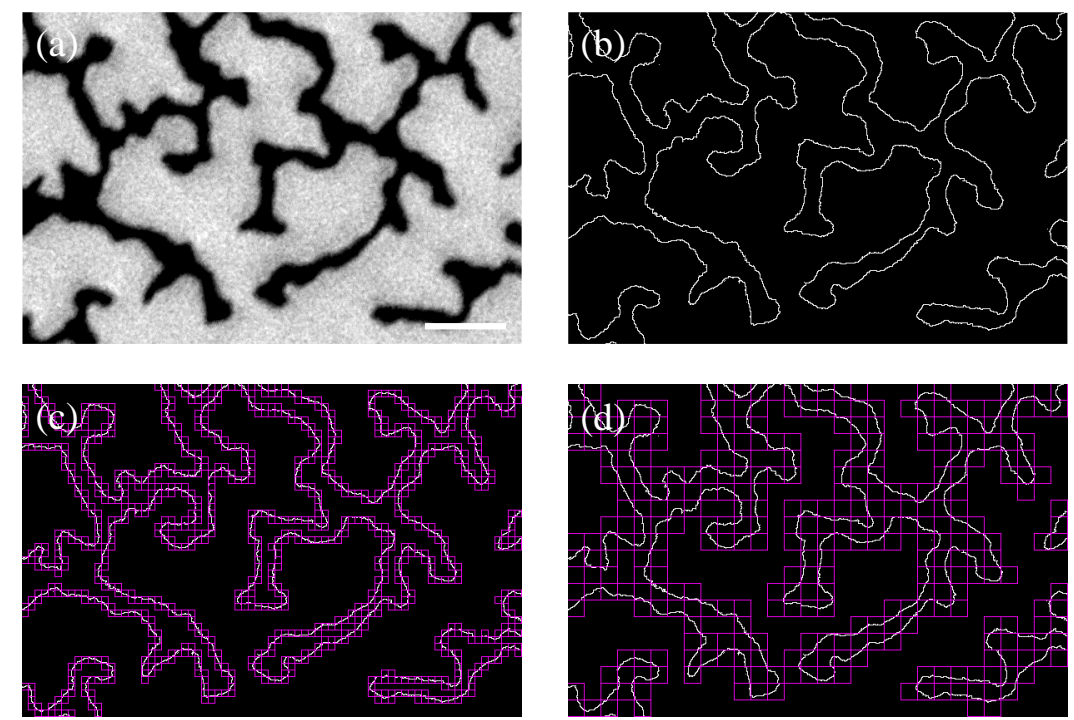

Figure 4.6: (a) Original image of magnetic domains. (b) Image after identifying the binary contour pattern. (c)-(d) Image showing the contour of magnetic domain covered by squares with size 8 and 20 pixels, respectively.

$$
D_{\mathrm{f}}=\frac{\ln (N(s))}{|\ln (s)|},
$$

where $s$ is the width of a square element used to cover the contour pattern and $N(s)$ is the number of elements needed to cover the contour pattern. For each image, this measurement was repeated for a range of element sizes $s$ and then use a linear fit on the $\ln (N(s)) v s|\ln (s)|$ graph (see e.g. Figure 4.7(a) to report $D_{\mathrm{f}}$ for this experimental condition. This allows us to quantify the complexity of the contour of the magnetic domains in these samples, with an image showing $D_{\mathrm{f}}=1$ representing a trivial straight line domain contour, while $D_{\mathrm{f}}$ close to 2 represents a highly complex domain contour such as a Peano Space-filling curve [131]. $D_{\mathrm{f}}$ of the image of Figure 4.7(a) is calculated using equation (4.5) for varying element size $s$ measured in pixels, as shown in Figure 4.7(a). A typical linear relationship has 
been observed in the $\ln (N(s))$ vs $|\ln (s)|$ plot, and $D_{\mathrm{f}}$ is 1.76. Applying this process to MOKE images taken at several different experimental conditions results in Figure 4.7(b), where the fractal dimension $D_{\mathrm{f}}$ vs the IP field was plotted.

Three qualitatively different regions can be identified directly from the MOKE images: labyrinth domains, coexistence of labyrinth domains and skyrmions, and only skyrmions. The colour of each data point shows the ratio $\gamma$ of the total area covered by the skyrmions compared with the area covered by the skyrmions and labyrinth domains. It should be noted that in fractal analysis only isolated skyrmions are counted as skyrmions, and a very small number of skyrmions that cannot be clearly resolved from each other might be ignored. With no IP field, i.e. $\mu_{0} H_{\mathrm{x}}=0 \mathrm{mT}, D_{\mathrm{f}}$ decreases from 1.82 to 1.26 with increasing $\mu_{0} H_{\mathrm{z}}$ as labyrinth domains collapse and the film magnetization saturates along the OP direction (see Figures 4.2(a)(c)). At low OP field $\mu_{0} H_{\mathrm{z}}=-0.8 \mathrm{mT}$, the value of $D_{\mathrm{f}}$ is constant for low IP fields $\mu_{0} H_{\mathrm{x}}$ and then begins to increase at high $\mu_{0} H_{\mathrm{x}}$, as the labyrinth domains start to gain finer, more complex features. For $\mu_{0} H_{\mathrm{z}}=-1.2 \mathrm{mT}, D_{\mathrm{f}}$ is almost constant up to $\mu_{0} H_{\mathrm{x}}=35 \mathrm{mT}$ and then increases with increasing $\mu_{0} H_{\mathrm{x}}$ : initially the labyrinth domains gain finer features (see Figure 4.2(d)) and above $35 \mathrm{mT}$ skyrmions are generated from labyrinth domains as well as from the uniformly ferromagnetic region (see Figure 4.2(g)). For $\mu_{0} H_{z}$ $=-1.6$ and $-2.0 \mathrm{mT}, D_{\mathrm{f}}$ first decreases as $\mu_{0} H_{\mathrm{x}}$ rises due to the disappearance of the labyrinth domains (see Figures 4.2(b) and (e)). Between $\mu_{0} H_{\mathrm{x}}=$ 25 and $30 \mathrm{mT}$, skyrmions are generated from collapse of the labyrinth domains (green shaded region of Figure 4.6(b)), however the value of $D_{\mathrm{f}}$ continuously decreases since more labyrinth domains disappear completely than collapse to create skyrmions. This can be reflected in the area ratio $\gamma$ at the lowest point of $D_{\mathrm{f}}$ where $\gamma$ is only $4.6 \%$ and $8.7 \%$ for $\mu_{0} H_{\mathrm{z}}=-1.6$ and $-2.0 \mathrm{mT}$, respectively. As $\mu_{0} H_{\mathrm{x}}$ is further increased, $D_{\mathrm{f}}$ increases rapidly due to a sharp increase in the generation of skyrmions. Interestingly, the lowest value of $D_{\mathrm{f}}$ for each curve coincides with a critical point separating 
two regions of skyrmion formation. In the labyrinth and skyrmion cohabitation regime (green area), for $\mu_{0} H_{\mathrm{x}}$ lower than the value where $D_{\mathrm{f}}$ reaches a minimum, skyrmions form only from the collapse of labyrinth domains (see Figures 4.3(a)-(c)). For $\mu_{0} H_{\mathrm{x}}$ above this point skyrmions nucleate out of the uniformly ferromagnetic region as well (see Figure 4.3(d)). This trend is even more stark when $\mu_{0} H_{z}=-2.3 \mathrm{mT}$, where the labyrinth domains shrink and completely disappear at the critical point $\mu_{0} H_{\mathrm{x}}=-35.5$ $\mathrm{mT}$, where a featureless magnetic image similar to Figure 4.2(f) was observed. At higher $\mu_{0} H_{\mathrm{x}}$, all the skyrmions nucleate directly from this featureless ferromagnetic region (see Figure 4.2(i)).

In summary, there are three distinct processes of domain transformation after sweeping the IP field, which were categorised using the fractal dimension. First, in the low OP field region $\left(\mu_{0} H_{\mathrm{z}}=-0.8\right.$ and $\left.1.2 \mathrm{mT}\right)$, $D_{\mathrm{f}}$ increases because the labyrinth domains gain finer features, and after a threshold IP field is reached, skyrmions are generated by the break up of the labyrinth domains. Second, $D_{\mathrm{f}}$ decreases due to the dominant effect of the disappearance of the labyrinth domains. Third, at the high IP field region above the critical point, $D_{\mathrm{f}}$ increases due to the appearance of skyrmions from the uniformly ferromagnetic region.

\subsubsection{Further analysis of skyrmions formed from the in- plane field}

Figure 4.8 shows the skyrmion density and nearest neighbour distance (NND) of the skyrmions as a function of the IP field at $\mu_{0} H_{z}=-2$ and $-2.3 \mathrm{mT}$, respectively. The skyrmion density increases with rising IP field, as shown in the black curve of Figures 4.8(a) and (b). It is known that the skyrmion density increases with an increase of the critical material parameter $\kappa=\pi D /\left(4 \sqrt{A_{\text {ex }} K_{\text {eff }}}\right)$, where $D$ is the DMI coefficient and $A_{\text {ex }}$ is the exchange stiffness [116, 122]. A disordered isolated skyrmions fabric progressively transforms into a skyrmion lattice when $0<\kappa<1$ shifts to 

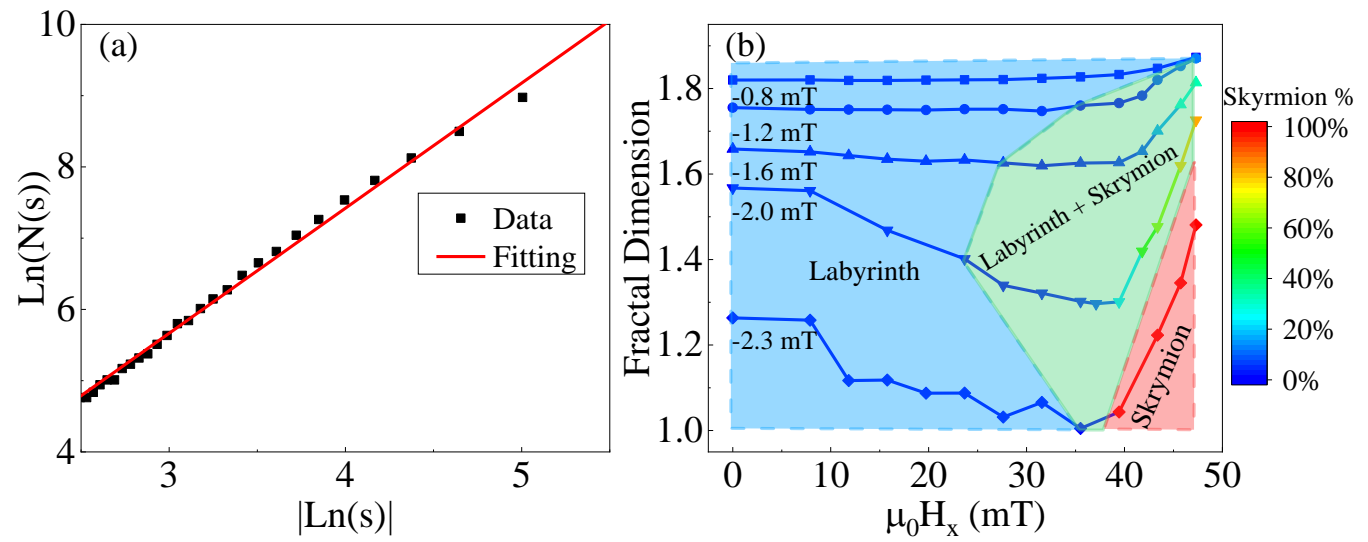

Figure 4.7: (a) Log-log plot of the number of elements and the element size for the domain patterns of Figure 4.2(a). The slope of fitting curve represents the fractal dimension. (b) Magnetic phase diagram related to the fractal dimension as a function of IP field under various OP field. The colour bar represents $\gamma$, the total fraction of skyrmions.
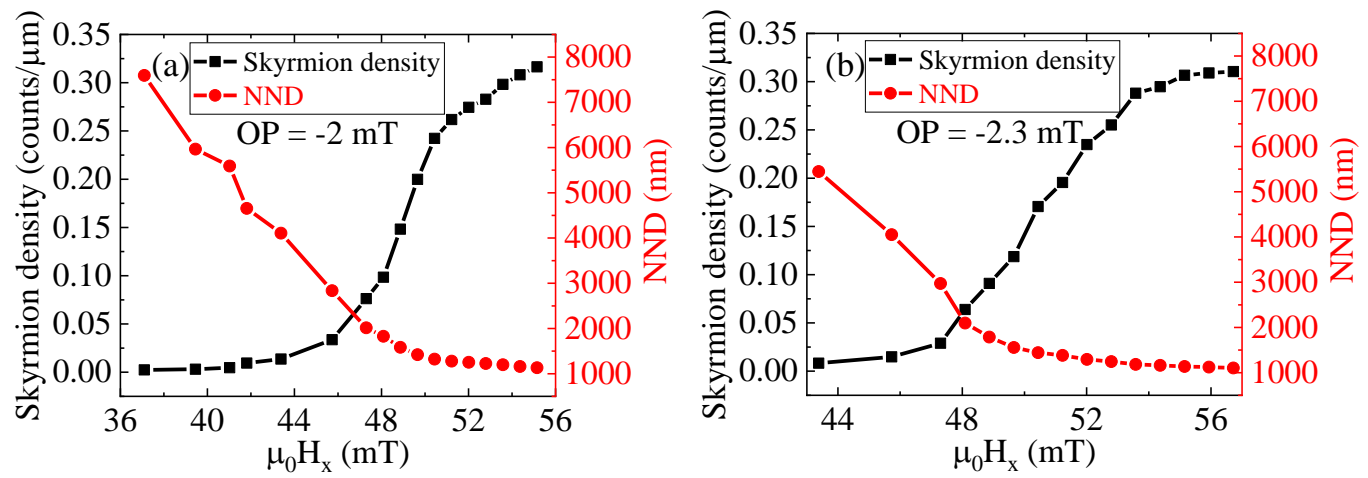

Figure 4.8: (a)-(b) The skyrmion density and NND as a function of IP field under $\mu_{0} H_{\mathrm{z}}=-2$ and $-2.3 \mathrm{mT}$, respectively.

$\kappa>1$ [46]. Generally, $D$ and $A_{\text {ex }}$ are fixed in a given system. However, the $K_{\text {eff }}$ in a perpendicularly magnetized material is weakened by applying an IP field, which leads to an increase of effective $\kappa$. For this system, the increase of skyrmion density agrees very well with this theory in the low IP field regime. However, the skyrmion density increases slowly or 
even reaches a plateau at high IP field, which may be explained by a repulsive skyrmion-skyrmion interaction [132], which inhibits the skyrmion density increasing beyond a certain value. Also, the NND as a function of IP field was measured, which defined as the distance from centre to centre of neighbouring skyrmions, as shown in the red curve of Figures 4.8(a) and (b). The value of NNDs at first decreases with increasing IP field, but at the same field where the density reaches a plateau, the NND value also approaches a constant. From the domain images, the skyrmions do not merge with each other at high fields. This indicates the presence of a repulsive interaction between the skyrmions in these thin films.

\subsection{Discussion and conclusions}

Given that skyrmions originate from the competition of exchange interaction, magnetic anisotropy and DMI; and that neither the exchange interaction nor the DMI in this system are expected to change in a magnetic field; the formation of labyrinth domains with only an OP field is due to the competition of PMA and DMI. When applying an IP field, the positive $K_{\text {eff }}$ becomes smaller, causing the PMA to gradually decrease with a rising IP field. Where an IP field was used to weaken the PMA, skyrmions are generated from collapsing labyrinth domains. When the IP field is large enough to reduce $K_{\text {eff }}$ so the film has very weak PMA, skyrmions are generated directly from the uniformly ferromagnetic region. In previous work $[133,134,70,135,136]$, the strength of the PMA in MgO/Heusler alloy/Pd trilayers is related to the thickness of the Heusler alloy layer. Films with slightly thicker layers of Pd were also produced, which result in smaller $K_{\text {eff }}$ values and hence the films have a weaker PMA - in these films skyrmions were able to generated by using just an OP field, as shown in Figure 3.6(c) and 3.7. The effect of the different Pd thickness and the effect of an IP field on the hysteresis loops is similar. The DMI constant $D$ is $0.46 \mathrm{~mJ} / \mathrm{m}^{2}$ in this system, which is at the intermediate strength. Previous 
research has shown that a small $D \sim 0.25 \mathrm{~mJ} / \mathrm{m}^{2}$ is enough to stabilize skyrmions in $\mathrm{Ta} / \mathrm{CoFeB} / \mathrm{MgO}$ with a weak $K_{\text {eff }} \sim 3.3 \times 10^{4} \mathrm{~J} / \mathrm{m}^{3}[137,115]$. It is known that reducing the $K_{\text {eff }}$ could lower the critical $D$ which is necessary to form non-collinear spin textures [138]. Using an IP field, the PMA in these multilayers were able to reduce to $K_{\text {eff }} \sim 2.3-2.6 \times 10^{4} \mathrm{~J} / \mathrm{m}^{3}$, reaching the regime where skyrmions are stabilized by the DMI.

In summary, the $\mathrm{MgO} / \mathrm{Mn}_{2} \mathrm{CoAl} / \mathrm{Pd}$ ultrathin films with PMA was fabricated, which show chiral labyrinth domains in an OP field due to the competition between PMA and DMI. With an IP field, skyrmions can be gradually generated from both collapse of the labyrinth domains and nucleation from uniformly ferromagnetic regions due to the reduction of

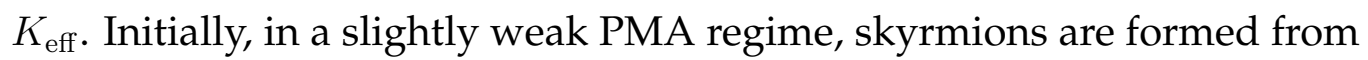
labyrinth domains. Further increasing the IP field reduces $K_{\text {eff }}$ which weakens the PMA and skyrmions start to form from uniformly ferromagnetic regions directly. By doing fractal analysis, a magnetic phase diagram was established that clearly identifies the two different types of skyrmion formation, as a function of fractal dimension and IP field. The processes of evolution of magnetic domains and skyrmions can be described by the fractal dimension. An increasing IP field causes an effective increase in the critical material parameter $\kappa$, which increases the skyrmion density up to a point beyond which the effect of a repulsive skyrmion-skyrmion interaction becomes dominant. These results show that skyrmions can be gradually generated with an assisted IP field and that fractal analysis is a potentially powerful tool to analyse the evolution of magnetic domains and skyrmions. 


\section{Chapter 5}

\section{Skyrmions generated by ionic liquid gating in $\mathrm{Mn}_{2} \mathrm{CoAl}$ ultrathin films}

\subsection{Introduction}

At chapter 4, the skyrmions can be generated by applying an external inplane magnetic field due to the change of effective magnetic anisotropy have discussed. Another method to engineer the magnetic anisotropy and DMI in thin films is electric field gating. Recently, the generation of skyrmions by this method has been demonstrated in various systems with PMA, like Pt/Co/oxide [139], Ta/CoFeB/TaO ${ }_{x}$ [140], Pt/CoNi multilayers [141] and IrMn/CoFeB/MgO [142] and due to the rise of DMI or reduction of PMA. However, these works employ a conventional field effect transistor (FET)-like structure using thick insulating gate dielectrics, like $\mathrm{SiO}_{2}$ or $\mathrm{HfO}_{2}$, with relatively small charge carrier densities resulting in a small capacitance and electric field [90]. Inevitably, skyrmions were only generated in thin films with a magnetic anisotropy close to the spinreorientation transition between the OP and IP direction at a large gate voltage. 
Instead of using a solid state dielectric, ionic liquid gating (ILG) using ionic liquids or ion gels has been demonstrated as a powerful method to effectively tune the magnetic anisotropy in $\mathrm{MgO} / \mathrm{FM} /$ heavy metal thin films [91, 92] and DMI [93]. Recently, using ILG, a high voltage-controlled magnetic anisotropy coefficient of $14.6 \mathrm{mT} \mathrm{V}^{-1}$ [92] and a DMI modification of $\sim 0.5 \mathrm{~mJ} \mathrm{~m}^{-2}$ [93] have been achieved. Thus, ILG can be a useful method to engineer magnetic interactions as mentioned above and generate skyrmions at a low gate voltage.

For ILG effect in ferromagnetic thin films, there are three potential mechanisms: electrostatic charging, magneto-ionic effect and electrochemical reaction. The electrostatic charging is due to charge accumulated at the surface which generates a volatile ultrahigh electric field, which could change the charge carrier density [91] and interfacial spin-orbit coupling of thin films [92] and thus induce a volatile behavior meaning that the magnetic state would be back to the initial state when the power is off. Magneto-ionic effect and electrochemical reaction are due to the ion diffusion [143] and redox reactions [144], respectively, which are expected to induce a nonvolatile effect meaning that the magnetic behavior of thin films can be retained once the voltage is switched off.

In this chapter, the $\mathrm{MgO} / \mathrm{Mn}_{2} \mathrm{CoAl} / \mathrm{Pd}$ ultrathin films with PMA by ILG were investigate. The ILG can generate both non-volatile and volatile skyrmions was demonstrated. Non-volatile skyrmions can be generated at a negative gate voltage in ultrathin films starting with either weak or intermediate PMA due to a magneto-ionic effect. Also volatile skyrmions can be generated at a small positive gate voltage after applying a large trigger gate voltage, which can be explained by electrostatic charge accumulation. All skyrmion generation modes happen at a non-skyrmion thickness regime demonstrating that ILG is a versatile technique to tune the magnetic anisotropy and DMI strength so that the magnetic texture can be designed and optimised without the need for combinatorial growth of thin films with sensitive magnetic parameters. 


\subsection{Results}

\subsubsection{Non-volatile skyrmion generation by ILG}

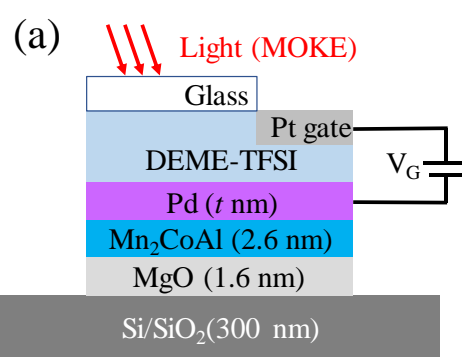

(c)
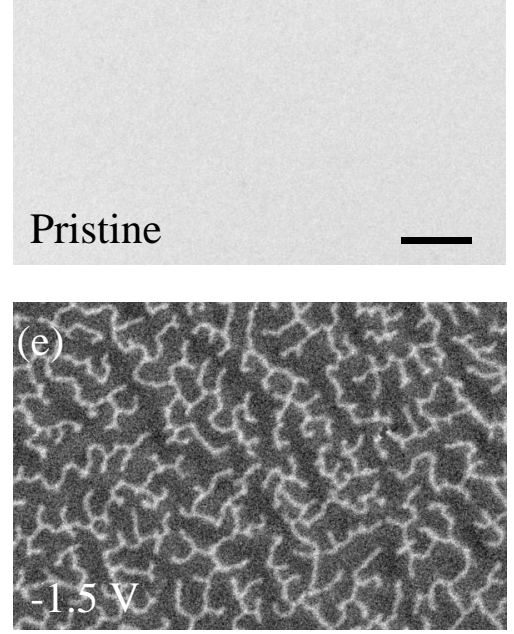
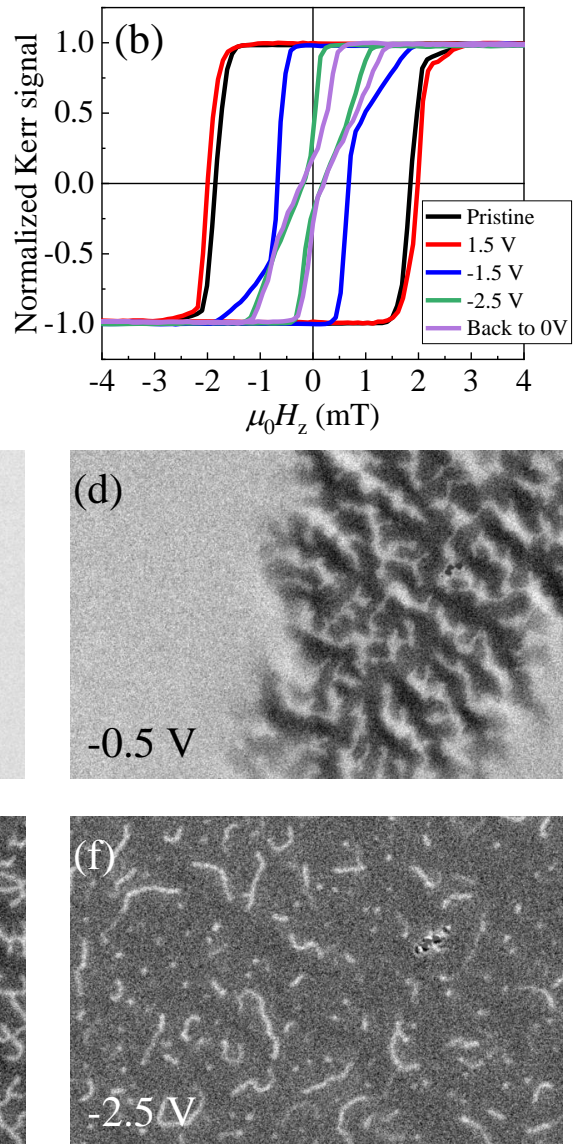

Figure 5.1: Schematic of device and gate-dependent magnetic characterization. (a) Schematic of ionic liquid device for $\mathrm{MgO} / \mathrm{Mn}_{2} \mathrm{CoAl} / \mathrm{Pd}$ stacks. (b) Gate dependence of hysteresis loops measured in an out-of-plane magnetic field at ambient temperature. (c)-(f) The corresponding magnetic domain images with $\mu_{0} H_{\mathrm{z}}=1.0 \mathrm{mT}$ at pristine, $-0.5,-1.5$, and $-2.5 \mathrm{~V}$, respectively. The scale bar in (c) corresponds $10 \mu \mathrm{m}$.

Figure 5.1(a) shows the device structure of $/ \mathrm{Mn}_{2} \mathrm{CoAl}$ trilayer with ionic liquid. A small droplet of ionic liquid, N,N-Diethyl-N-methyl-N-(2- 
methox yethyl)ammoniumbis-(trifluoromethylsulfonyl)imide (DEME-TFSI), is used as the electrolyte connecting the Pt gate electrode and the surface of sample. Samples are charged for 5 minutes at each voltage to reach the equilibrium of carriers, unless indicated otherwise. These gating experiments are carried out in ambient atmosphere at room temperature.

The magnetic hysteresis loops for the $\mathrm{MgO}(1.6 \mathrm{~nm}) / \mathrm{Mn}_{2} \operatorname{CoAl}(2.6 \mathrm{~nm}) /$ $\operatorname{Pd}(3.2 \mathrm{~nm})$ ultrathin film were measured by polar MOKE in a sweeping OP magnetic field at ambient temperature, as shown in Figure 5.1. Figures 5.1(c)-(f) show the corresponding magnetic domains under an OP field $-1 \mathrm{mT}$ after sweeping from positive magnetic saturation field. This pristine sample shows a clear PMA with a coercive field of $\mu_{0} H_{\mathrm{c}}=1.85 \mathrm{mT}$ (Figure 5.1(b)) and a uniform magnetization at $-1 \mathrm{mT}$ (Figure 5.1(c)). The anisotropy field $\mu_{0} H_{\mathrm{k}}$ is found to be $163 \mathrm{mT}$ fitted by the Stoner-Wohlfarth model. Thus, the effective magnetic anisotropy constant $K_{\text {eff }}=(1 / 2) \mu_{0} H_{\mathrm{k}} M_{\mathrm{s}}$ is $2.56 \times 10^{4} \mathrm{~J} \mathrm{~m}^{-3}$, where $M_{\mathrm{s}}=3.15 \times 10^{5} \mathrm{~A} \mathrm{~m}^{-1}$ is the saturation magnetization as mentioned in the chapter $3 . D$ can be calculated as $0.36 \mathrm{~mJ} \mathrm{~m}^{-2}$ for the film in the pristine state by using the same method in the chapter 4. Under this magnitude of DMI, Néel-type chiral domain walls are expected, which have been confirmed by using the asymmetric domain wall expansion method, as shown in the chapter 4 .

Then, a gate voltage $\left(\mathrm{V}_{\mathrm{G}}\right)$ on the stack was started to apply. At $\mathrm{V}_{\mathrm{G}}=1.5$ $\mathrm{V}, \mu_{0} H_{\mathrm{c}}$ slightly increases to $2 \mathrm{mT}$, and there is no change to the magnetic domains at $-1 \mathrm{mT}$. By changing $\mathrm{V}_{\mathrm{G}}$ to $-0.5 \mathrm{~V}$, the labyrinth domains start to nucleate from the uniform ferromagnetic state (see Figure 5.1(d)). At a negative $\mathrm{V}_{\mathrm{G}}=-1.5 \mathrm{~V}, \mu_{0} H_{\mathrm{c}}$ significantly shrinks to $0.67 \mathrm{mT}$, and the magnetic moment transition is not sharp near the saturation regime. What's more, only labyrinth domains can be observed (see Figure 5.1(e)). By continuing to increase $\mathrm{V}_{\mathrm{G}}$ to $-2.5 \mathrm{~V}, \mu_{0} H_{\mathrm{c}}$ shrinks to $0.18 \mathrm{mT}$, and the remanent state is not saturated anymore indicating that the magnetic anisotropy approaches the spin-reorientation transition between the OP and IP direction. Interestingly, skyrmions with average diameter $\sim 1 \mu \mathrm{m}$ can be gener- 
ated and coexist with labyrinth domains, as shown in Figure 5.1(f). When the voltage is set back to $0 \mathrm{~V}, \mu_{0} H_{\mathrm{c}}$ stays the same as it was with $\mathrm{V}_{\mathrm{G}}=$ $-2.5 \mathrm{~V}$ and the remanent state is still unsaturated showing this ILG effect is non-volatile.

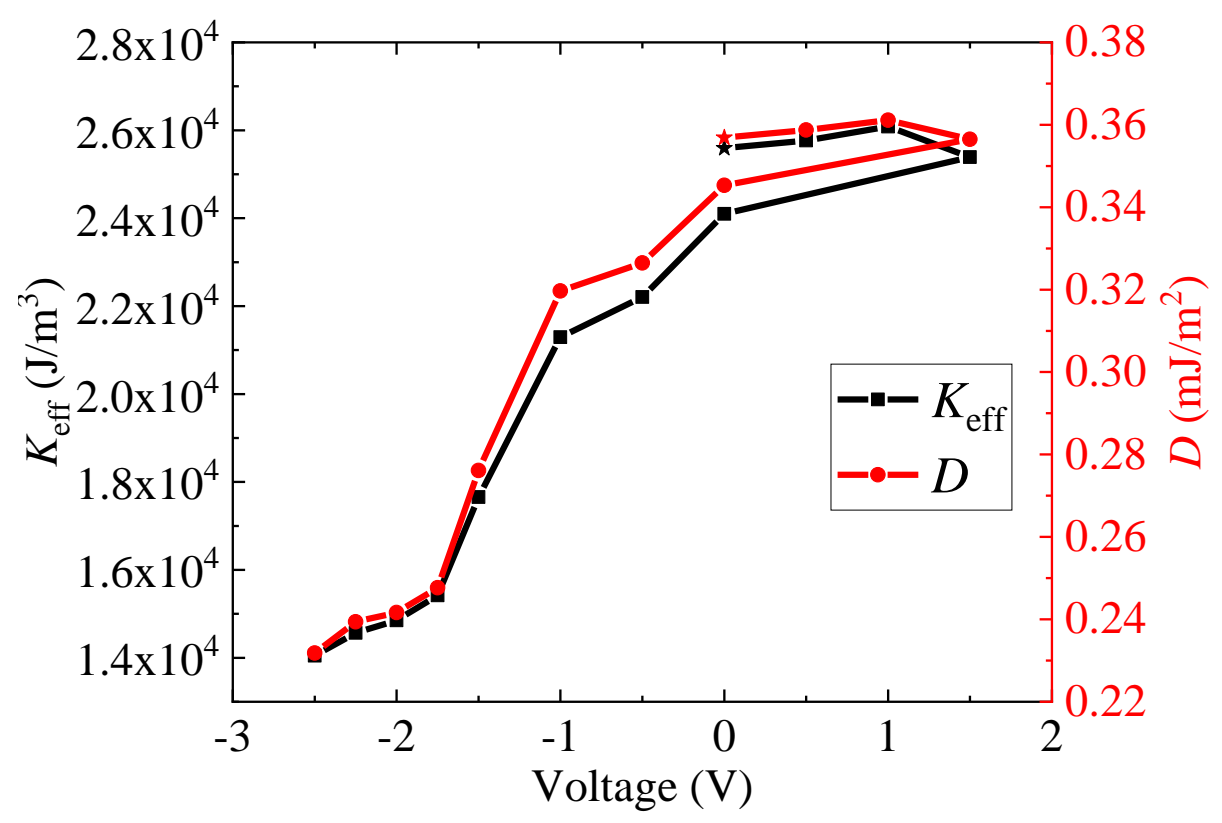

Figure 5.2: Effective magnetic anisotropy, $K_{\text {eff }}$, and DMI coefficient $D$ as a function of gate voltage. The star corresponds to the pristine state.

Given that skyrmions originate from the competition of DMI and magnetic anisotropy, $K_{\text {eff }}$ and $D$ at various gate voltages were able to calculates, as shown in Figure 5.2. The influence on $K_{\text {eff }}$ is very small at positive gate voltage. However, $K_{\text {eff }}$ significantly decreases with increasing negative gate voltage. Especially, at $-2.5 \mathrm{~V}, K_{\text {eff }}$ drops to $1.4 \times 10^{4} \mathrm{~J} \mathrm{~m}^{-3}$ with a remarkable anisotropy field tunability of $29.3 \mathrm{mT} \mathrm{V}^{-1}$, which is twice the previous record $\left(14.6 \mathrm{mT} \mathrm{V}^{-1}\right)$ [92]. The change in trend of $D$ as a function of gate voltage is similar to the one of the $K_{\text {eff. }}$ At $\mathrm{V}_{\mathrm{G}}=-2.5 \mathrm{~V}$, the $D$ decreases by $35 \%$ to $0.23 \mathrm{~mJ} \mathrm{~m}^{-2}$. Usually, to obtain skyrmions in ultrathin films, samples should exhibit a weak $K_{\text {eff }}$ or a comparatively large $D[138,50]$. Even though the value of $D$ decreases at negative voltage, the 
rate of $K_{\text {eff }}$ decrease is more than the one of $D$. A very small value $K_{\text {eff }}$ has been reached at $-2.5 \mathrm{~V}$, where the magnetic anisotropy closes to the spinreorientation transition in which skyrmions can be easy annihilated [117]. Previous research has shown that a $D \sim 0.25 \mathrm{~mJ} \mathrm{~m}^{-2}$ is enough to stabilize skyrmions in $\mathrm{Ta} / \mathrm{CoFeB} / \mathrm{MgO}$ with a $K_{\text {eff }} \sim 3.3 \times 10^{4} \mathrm{~J} \mathrm{~m}^{-3}[137,115]$. By using ILG, the PMA were able to reduced in these thin films to a very weak $K_{\text {eff }}=1.4 \times 10^{4} \mathrm{~J} \mathrm{~m}^{-3}$ with a relatively small $D=0.23 \mathrm{~mJ} \mathrm{~m}^{-2}$, reaching the regime where skyrmions are stabilized by the DMI. The magnetic configuration of the skyrmions is expected to be Néel-type, the same as that of the domain walls, which is similar to other PMA trilayers in the literature [47].

\subsubsection{Non-volatile skyrmion generation in intermediate PMA trilayers}

For thin films with a small $\mu_{0} H_{\mathrm{c}}$ and weak $K_{\text {eff }}$, a single gate voltage, less than $-2.5 \mathrm{~V}$, is enough to reduce PMA into a region where skyrmions can be generated, as shown above. It should be noted that, in this work, thin films with a weak PMA is defined that the magnetic anisotropy closes to the spin-reorientation transition between the OP and IP direction. In the case of samples with intermediate or strong $K_{\text {eff }}$ which usually show a large coercive field, one has to apply a large single voltage to create skyrmions. However, electrochemical reactions can happen at large voltages [145] which could significantly change the properties or even damage the sample.

To generate skyrmions in a thin film with an as-grown intermediate PMA, an alternative gating method was employed by repeatedly cycling the gate voltage from $-2.5 \mathrm{~V}$ to $1 \mathrm{~V}$. A trilayer consisting of $\mathrm{MgO}(1.6$ $\mathrm{nm}) / \mathrm{Mn}_{2} \operatorname{CoAl}(2.6 \mathrm{~nm}) / \mathrm{Pd}(2.8 \mathrm{~nm})$ was firstly studied, which has an intermediate strength PMA with $K_{\text {eff }}=5.76 \times 10^{4} \mathrm{~J} \mathrm{~m}^{-3}$ and $\mu_{0} H_{\mathrm{c}}=8.46 \mathrm{mT}$, as shown in Figure 5.3(a). Due to these large magnetic parameters, the pe- 

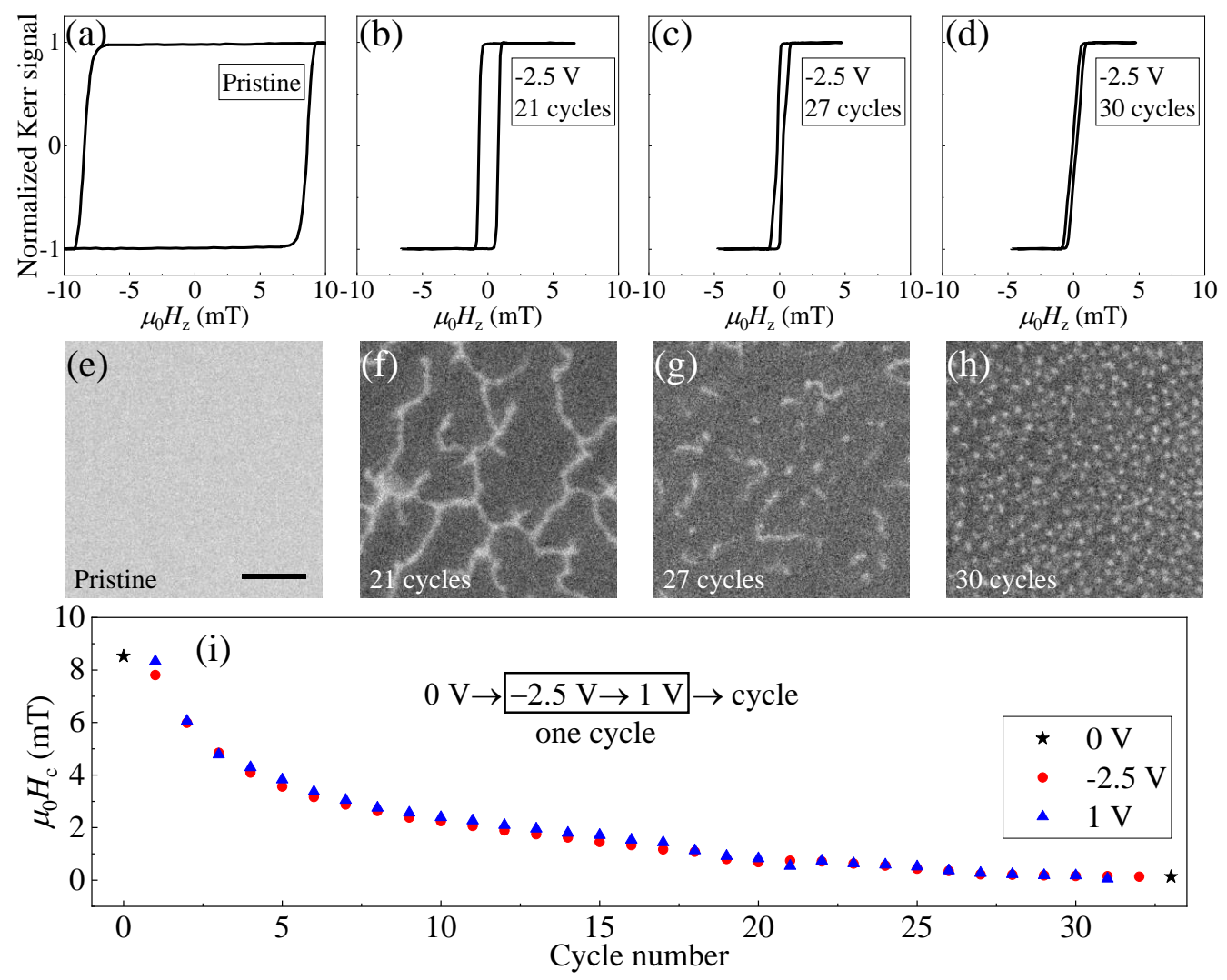

Figure 5.3: Evolution of hysteresis loops and magnetic domains. (a) Hysteresis loop of $\mathrm{MgO}(1.6 \mathrm{~nm}) / \mathrm{Mn}_{2} \mathrm{CoAl}(2.6 \mathrm{~nm}) / \mathrm{Pd}(2.8 \mathrm{~nm})$ trilayer at pristine state. (b)-(d) Hysteresis loops at $-2.5 \mathrm{~V}$ after cycling 21, 27, and 30 times, respectively. (e)-(h) The corresponding magnetic domain images, with $\mu_{0} H_{\mathrm{z}}=0.66 \mathrm{mT}$, captured from (a)-(d), respectively. The scale bar in (e) corresponds $10 \mu \mathrm{m}$. (i) $\mu_{0} H_{\mathrm{c}}$ as a function of cycle number for 1 and $-2.5 \mathrm{~V}$, respectively.

riodic demagnetized domains cannot be obtained and thus the DMI with the method mentioned in subchapter 4.2 .3 cannot be calculated.

Figures 5.3(b)-(d) and (f)-(h) show the magnetic hysteresis loops at $-2.5 \mathrm{~V}$ after different numbers of cycles, and the corresponding magnetic morphology at $0.66 \mathrm{mT}$, respectively. Essentially, $\mu_{0} H_{\mathrm{c}}$ decreases with repeated cycling, and finally tends towards saturated, which resembles the 
training effect of exchange bias in antiferromagnet/ferromagnet bilayers, in which the exchange bias field gradually degrades upon repeated magnetic field cycling [146]. For instance, after cycling 21 times, a square loop still can be observed, but $\mu_{0} H_{\mathrm{c}}$ significantly decreases to $0.68 \mathrm{mT}$, and labyrinth domains nucleate from the uniform ferromagnetic state, as shown in Figures 5.3(b) and (f), respectively. For 27 cycles, the shape of the hysteresis loop looks similar to the green loop in Figure 5.1(b) after applying $\mathrm{V}_{\mathrm{G}}=-2.5 \mathrm{~V}$. Both of them exhibit similar magnetic morphology in which the coexistence of skyrmions and labyrinth domains can be observed (compare Figure 5.3(g) to Figure 5.1(f)). After $30 \mathrm{cy}-$ cles, $K_{\text {eff }}$ decreases to $1.44 \times 10^{4} \mathrm{~J} \mathrm{~m}^{-3}$ with a giant anisotropy field tunability of $109.8 \mathrm{mT} \mathrm{V}^{-1}$, and only skyrmions can be observed, as shown in Figure 5.3(h). Figure 5.3(i) shows the evolution of $\mu_{0} H_{\mathrm{c}}$ at -2.5 or $1 \mathrm{~V}$ by increasing cycles. Before 20 cycles, the value of $\mu_{0} H_{\mathrm{c}}$ decreases at $\mathrm{V}_{\mathrm{G}}=-2.5 \mathrm{~V}$ after each cycle, and then recovers a bit at $\mathrm{V}_{\mathrm{G}}=1 \mathrm{~V}$, which is in line with the results of Figure 5.1(b). After 20 cycles, $\mu_{0} H_{c}$ decreases slowly and tends towards saturation. The value of $\mu_{0} H_{\mathrm{c}}$ does not change by setting the voltage back to $0 \mathrm{~V}$ demonstrating this ILG behaviour is non-volatile.

\subsubsection{Volatile skyrmion generation by ionic liquid gating}

It is also possible to use ILG to change the magnetic anisotropy and coercive field and generate skyrmions in a volatile mode, i.e. the skyrmions disappear when the gate voltage is removed. $\mathrm{A} \mathrm{MgO}(1.6 \mathrm{~nm}) / \mathrm{Mn}_{2} \mathrm{CoAl}-$ $(2.6 \mathrm{~nm}) / \mathrm{Pd}(3.2 \mathrm{~nm})$ ultrathin film was studied, which has an initial anisotropy close to the sample of Figures 5.1-5.2, i.e. weak anisotropy. A large trigger voltage of $\mathrm{V}_{\mathrm{G}}=-3 \mathrm{~V}$ was firstly applied for charging 2 minutes, and then reduce $\mathrm{V}_{\mathrm{G}}$ to $-0.5 \mathrm{~V}$. The square hysteresis loop indicates a PMA, as shown in Figure 5.4(a). Subsequently, a positive $\mathrm{V}_{\mathrm{G}}=1.5 \mathrm{~V}$ is applied. Interestingly, the square loop disappears indicating that the PMA is entirely 

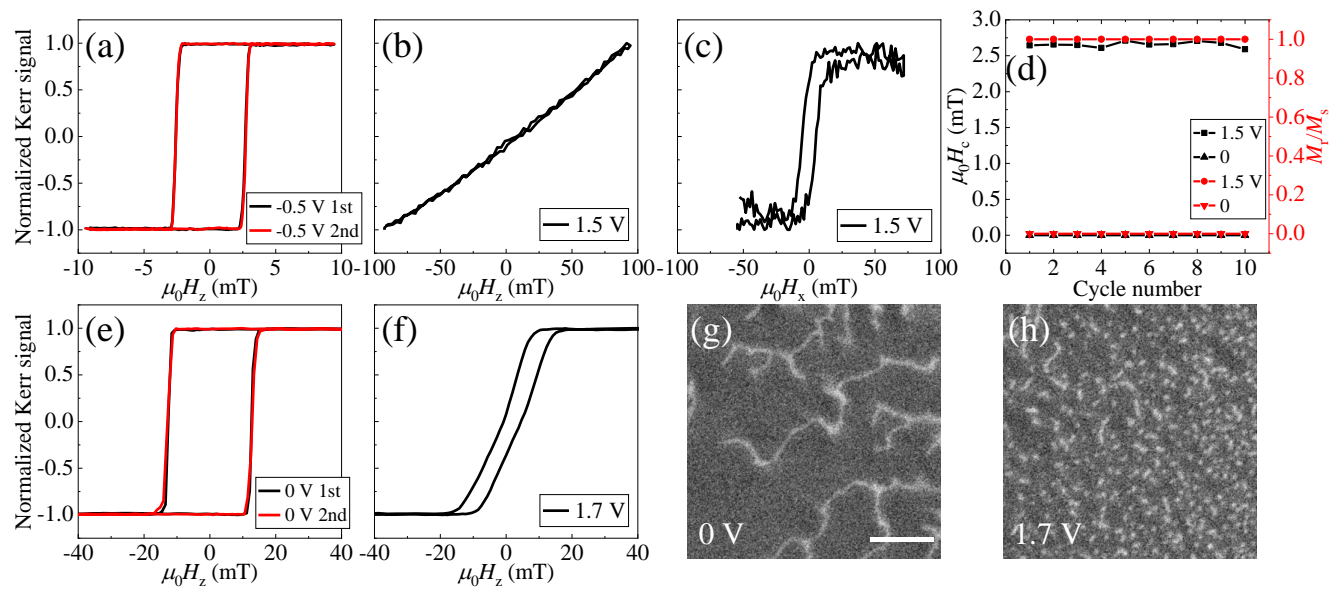

Figure 5.4: Volatile gating effect for hysteresis loops and magnetic domains. (a)(b) Hysteresis loops measured at -0.5 and $1.5 \mathrm{~V}$, respectively. (c) Longitudinal hysteresis loop as a function of in-plane magnetic field at $1.5 \mathrm{~V}$. (d) $\mu_{0} H_{\mathrm{c}}$ and $M_{\mathrm{r}} / M_{\mathrm{S}}$ as a function of cycle number at 0 and $1.5 \mathrm{~V}$, respectively. (e)-(f) Hysteresis loops measured at 0 and $1.7 \mathrm{~V}$, respectively. $(\mathrm{g})-(\mathrm{h})$ The corresponding magnetic domain images, with $\mu_{0} H_{\mathrm{z}}=1.1 \mathrm{mT}$, captured from (e)-(f), respectively.

lost (see Figure 5.4(b)). To prove that the magnetic moments rotate to the IP direction rather than the magnetization has been destroyed, the in situ longitudinal geometry of MOKE measurement is performed by sweeping an IP magnetic field. Figure 5.4(c) shows the longitudinal Kerr signal of the gated thin film at $1.5 \mathrm{~V}$. A clear loop can be observed demonstrating that a switch of the anisotropy from the OP to IP direction is achieved . When $\mathrm{V}_{\mathrm{G}}$ is set back to $-0.5 \mathrm{~V}$, the PMA is spontaneously recovered with a loop which is indistinguishable from the first $-0.5 \mathrm{~V}$ loop, as shown in the red curve in Figure 5.4(a). Figure 5.4(d) presents the reversibility of $\mu_{0} H_{\mathrm{c}}$ and the ratio of the remnant magnetization $\left(M_{\mathrm{r}}\right)$ to $M_{\mathrm{s}}$ as $\mathrm{V}_{\mathrm{G}}$ is cycled 10 times between $1.5 \mathrm{~V}$ and $-0.5 \mathrm{~V}$. Both $\mu_{0} H_{\mathrm{c}}$ and $M_{\mathrm{r}} / M_{\mathrm{s}}$ can be reversibly and non-destructively toggled into the IP direction with a small positive voltage. Further, skyrmions can be observed by using this charge method. Again, a trigger $-3 \mathrm{~V}$ was applied, and then back to $0 \mathrm{~V}$. The sam- 
ple starts with a PMA, as shown in Figure 5.4(e). After that, a positive $\mathrm{V}_{\mathrm{G}}$ $=1.7 \mathrm{~V}$ was applied, and one obtains a hysteresis loop with a shape that looks similar to the green one in Figure 5.1(b), which exhibits skyrmions. Figures 5.4(g) and (h) show the corresponding domain images under $\mu_{0} H_{z}$ $=1.1 \mathrm{mT}$, at 0 and $1.7 \mathrm{~V}$ respectively. Labyrinth domains can be observed at $0 \mathrm{~V}$, and then skyrmions are created at $1.7 \mathrm{~V}$. By setting $\mathrm{V}_{\mathrm{G}}$ back to 0 $\mathrm{V}$, the PMA is totally recovered, as shown in the red loop in Figure 5.4(e), showing this ILG behaviour is volatile.

\subsubsection{Surface analysis}

It is well known that the ILG modification is related to the electrostatic charging, magneto-ionic and electrochemical reaction [90]. To understand the potential mechanisms in this study, XPS is employed to characterize the oxidation or chemical state information of these ultrathin films at different ILG methods, as shown in Figure 5.5. Figures 5.5(a) and (b) show scans of the regions for $\mathrm{Pd} 3 d$ and $3 p_{3 / 2}$ core-levels, respectively, and a corresponding peak fitting for the weak PMA sample in the pristine state. The fits to the XPS results use the $\mathrm{Pd} 3 d_{5 / 2}$ and $3 p_{3 / 2}$ peaks that are in centered at $335.0 \mathrm{eV}$ and $531.4 \mathrm{eV}$, respectively [147]. The spectrum presented in Figure 5.5(a) shows a spin-orbit split doublet consistent with metallic Pd, with the $3 d_{5 / 2}$ and $3 d_{3 / 2}$ appearing as well separated peaks. To higher binding energy, there is a second species present, with weak peaks at $341.5 \mathrm{eV}$ and $336.3 \mathrm{eV}$, respectively, representing $\mathrm{O}-\mathrm{Pd}$ bonds [148] suggesting there is a very thin native oxide $\mathrm{PdO}_{x}$ layer. To confirm the oxidation of $\mathrm{Pd}$, the $\mathrm{O} 1 s$ spectrum is scanned, although it is very close to the $3 p_{3 / 2}$ line in Figure 5.5(b). One can observe a clear shoulder at $529.0 \mathrm{eV}$ on the low binding energy side of the $\mathrm{Pd} 3 p_{3 / 2}$ peak, which is consistent with an $\mathrm{O} 1 s$ peak, as shown in Figure 5.5(b).

It should be noted that there is no evidence that this $\mathrm{O} 1 \mathrm{~s}$ peak could be derived from the $\mathrm{MgO}$ or $\mathrm{SiO}_{2}$ layers, as no evidence for either $\mathrm{Mg}$ or 

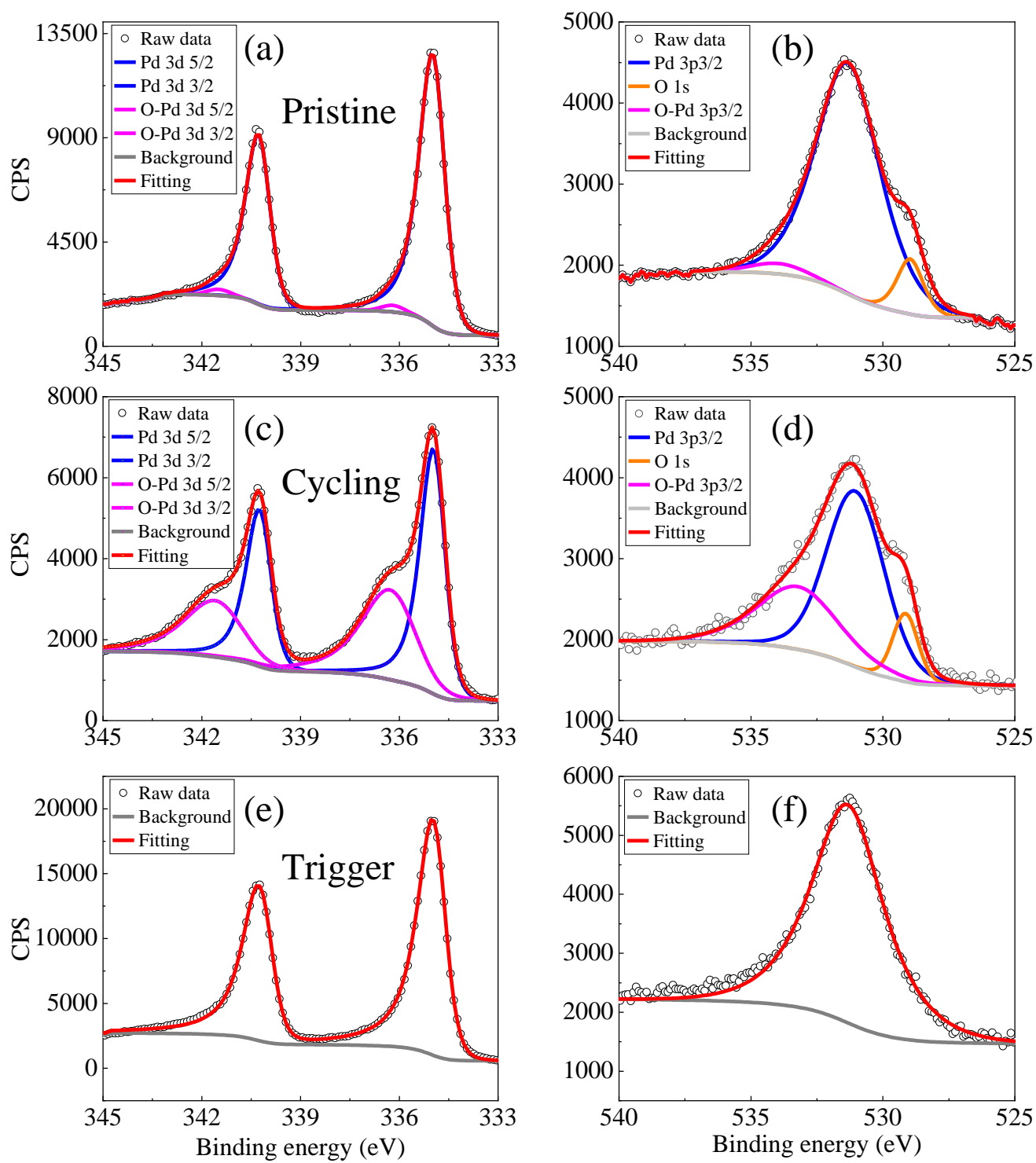

Figure 5.5: The XPS spectra of trilayers at various gating conditions. The XPS spectra of (a)-(b) pristine sample, (c)-(d) sample after cycling 30 times to skyrmion region, (e)-(f) sample after giving a trigger, $-3 \mathrm{~V}$.

Si was observed in the whole arrange of survey spectra. A simple consideration of the escape depth of an electron also precludes the oxygen observed here from originating in either of these layers. The mean escape 
depth of $\mathrm{O} 1 s$ is smaller than $2.42 \mathrm{~nm}$ [149] while both $\mathrm{MgO}$ and $\mathrm{SiO}_{2}$ are covered by $2.6 \mathrm{~nm} \mathrm{Mn}{ }_{2} \mathrm{CoAl}$ and 2.8-3.2 nm Pd layers. For the intermediate PMA sample, after cycling the gate voltage from $-2.5 \mathrm{~V}$ to $1 \mathrm{~V}$ more than 30 times, there are obviously broad peaks of O-Pd bond appearing at the same binding energy as they do in the pristine sample, as shown in Figures 5.5(c) and (d). Also, the peak of the $\mathrm{O} 1 \mathrm{~s}$ spectrum can now be clearly observed. However, after applying a large trigger voltage of $-3 \mathrm{~V}$, only pure $\mathrm{Pd} 3 d$ and $3 p$ peaks are observed, suggesting the native oxide $\mathrm{PdO}_{x}$ layer is not longer present, as shown in Figures 5.5(e) and (f).

\subsection{Discussion and conclusions}

Here, referring to the XPS measurements, the mechanisms that result in the non-volatile and volatile skyrmion generation by ILG at different charge methods will be discussed.

It is known that there is a considerable amount of residual water ( 391 p.p.m. by mass) in the hydrophobic ionic liquid DEME-TFSI [150]. In this situation, the $\mathrm{O}^{2-}$ or $\mathrm{H}^{+}$ions can be driven into thin films by ILG with a bias voltage due to the $\mathrm{H}_{2} \mathrm{O}$ hydrolysis in the ambient atmosphere above $1.23 \mathrm{~V}$ [151], therefore the phase [152, 150], and magnetic anisotropy [153] can be changed.

For the weak PMA thin film after applying a negative gate voltage, the $\mathrm{O}^{2-}$ ions from the hydrolysed water are driven towards the Pd layer and form a slightly thicker $\mathrm{PdO}_{x}$ layer, inducing the reduction of $\mu_{0} H_{\mathrm{c}}$. At -2.5 $\mathrm{V}$, both PMA and DMI decrease to a critical value where skyrmions can be formed. On the other hand, by applying a comparatively small positive voltage where part of the $\mathrm{O}^{2-}$ ions can be pumped out, the value of $\mu_{0} H_{\mathrm{c}}$ can partially be reversed.

For the sample with intermediate PMA by cycling gate voltage, after the first couple of $-2.5 \mathrm{~V}$ cycles, $\mu_{0} H_{\mathrm{c}}$ decreases rapidly as the $\mathrm{O}^{2-}$ ions easily diffuse into the Pd layer (see Figure 5.3(i)). After more cycles, the 
$\mu_{0} H_{\mathrm{c}}$ decreases more slowly and tends towards saturation, suggesting that it becomes harder for the $\mathrm{O}^{2-}$ ions to diffuse deeper into the $\mathrm{Pd}$ and, finally stabilizes at $-2.5 \mathrm{~V}$ after 30 times cycling. Here, after applying $1 \mathrm{~V}, \mu_{0} H_{\mathrm{c}}$ could partially reverse meaning there are still residual $\mathrm{O}^{2-}$ ions that cannot be removed from the $\mathrm{Pd}$ layer. In other words, the $\mathrm{PdO}_{x}$ layer thickness increases after each cycle until a maximum thickness is reached and the change in anisotropy saturates, which can be confirmed by XPS results Figures 5.5(c) and (d).

Essentially, for the first two ILG methods, where the maximum gate voltage applied is $-2.5 \mathrm{~V}$, the mechanism is the magneto-ionic effect because the $\mathrm{O}^{2-}$ ions diffuse into the surface and oxidise the Pd layer, which reduces the $K_{\text {eff }}$ and DMI and thus generates skyrmions in a non-volatile mode. To understand the progressive oxidation process of Pd layer due to oxygen diffusion, the depth profile of XPS has been performed, as shown in Figure 5.6. The spectra are recorded after every $30 \mathrm{~s} \mathrm{Ar}^{+}$etching. It should be noted that the $\mathrm{Pd} 3 d$ peak still exists after $240 \mathrm{~s} \mathrm{Ar}^{+}$etching suggesting that $\mathrm{Pd}$ layer has not been totally etched away. Since the native $\mathrm{PdO}_{x}$ is very thin, the $\mathrm{O} 1 s$ peak disappears after $30 \mathrm{~s} \mathrm{Ar}^{+}$etching, as shown in Figure 5.6(b).

However, for the thin film has been voltage cycling 30 times, a $90 \mathrm{~s}$ $\mathrm{Ar}^{+}$etching is required to remove the $\mathrm{O} 1 s$ peak (see Figure 5.6(d)) indicating that a thick $\mathrm{PdO}_{x}$ is formed and $\mathrm{O}^{2-}$ ions do not penetrate into the $\mathrm{Mn}_{2} \mathrm{CoAl}$ layer. Figure 5.7 shows the XPS spectra of $\mathrm{Co}, \mathrm{Mn}$ and $\mathrm{Al}$, respectively. The peaks obtained from different charging method, cycling and trigger, are same as those of pristine state. Thus, the $\mathrm{O}^{2-}$ ions only diffuse into the Pd layer and do not reach the $\mathrm{Mn}_{2} \mathrm{CoAl}$ layer. Also, the electrochemical reaction effect can be ruled out because the hysteresis loops are unchanged after charging for 5, 28 and 44 minutes at $-2.5 \mathrm{~V}$, respectively (see Figure 5.8).

For the sample after applying a trigger voltage of $-3 \mathrm{~V}$, the lack of a peak in the XPS spectrum showing O-Pd bond, as shown in Figures 5.5(e) 

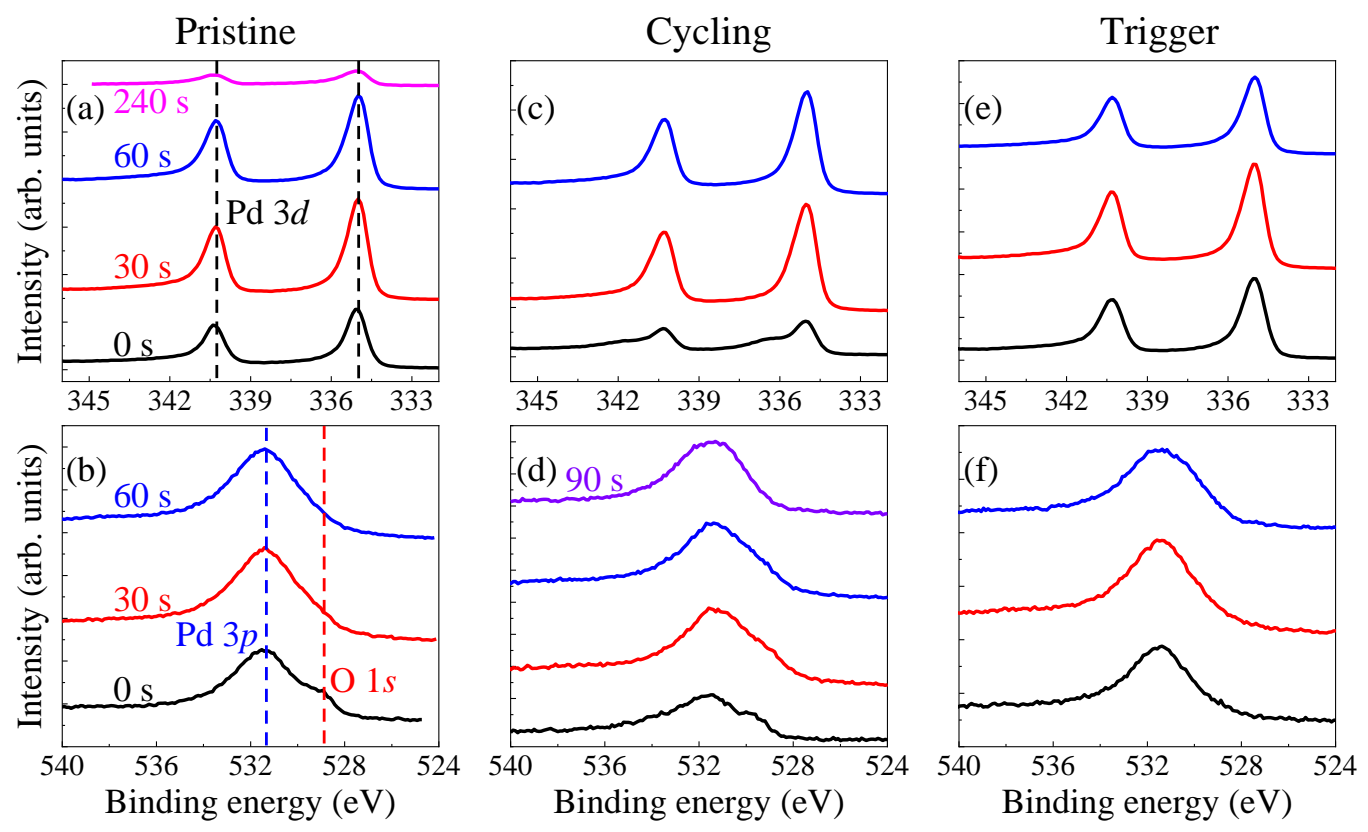

Figure 5.6: The XPS spectra of $\mathrm{Pd}$ and $\mathrm{O}$ after Ar ions etching 0, 30, 60, 90, and 240 $\mathrm{s}$, respectively, at (a)-(b) pristine state, (c)-(d) cycling 30 times, (e)-(f) $-3 \mathrm{~V}$ trigger.
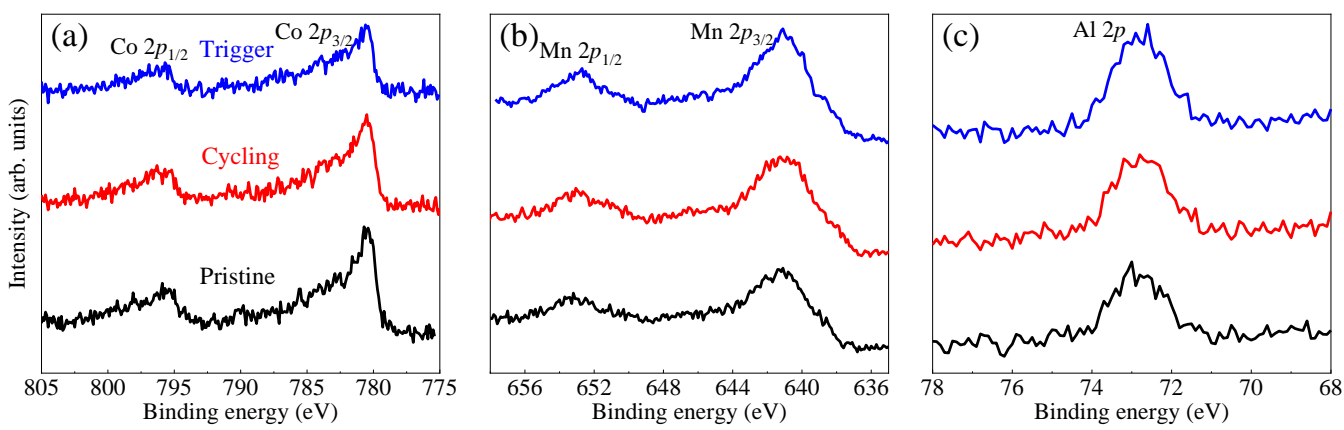

Figure 5.7: The XPS spectra of $\mathrm{Co}, \mathrm{Mn}$ and $\mathrm{Al}$ for pristine state, cycling 30 times, and $-3 \mathrm{~V}$ trigger, respectively, after Ar ions etching $120 \mathrm{~s}$.

and (f), indicates that the native oxide $\mathrm{PdO}_{x}$ layer in the pristine state is etched away as the edge of the electrochemical potential window of DEME-TFSI ( $-3 \mathrm{~V}$ to $2.7 \mathrm{~V}$ ) [154] can be reached. Thus, the ionic liquid is 


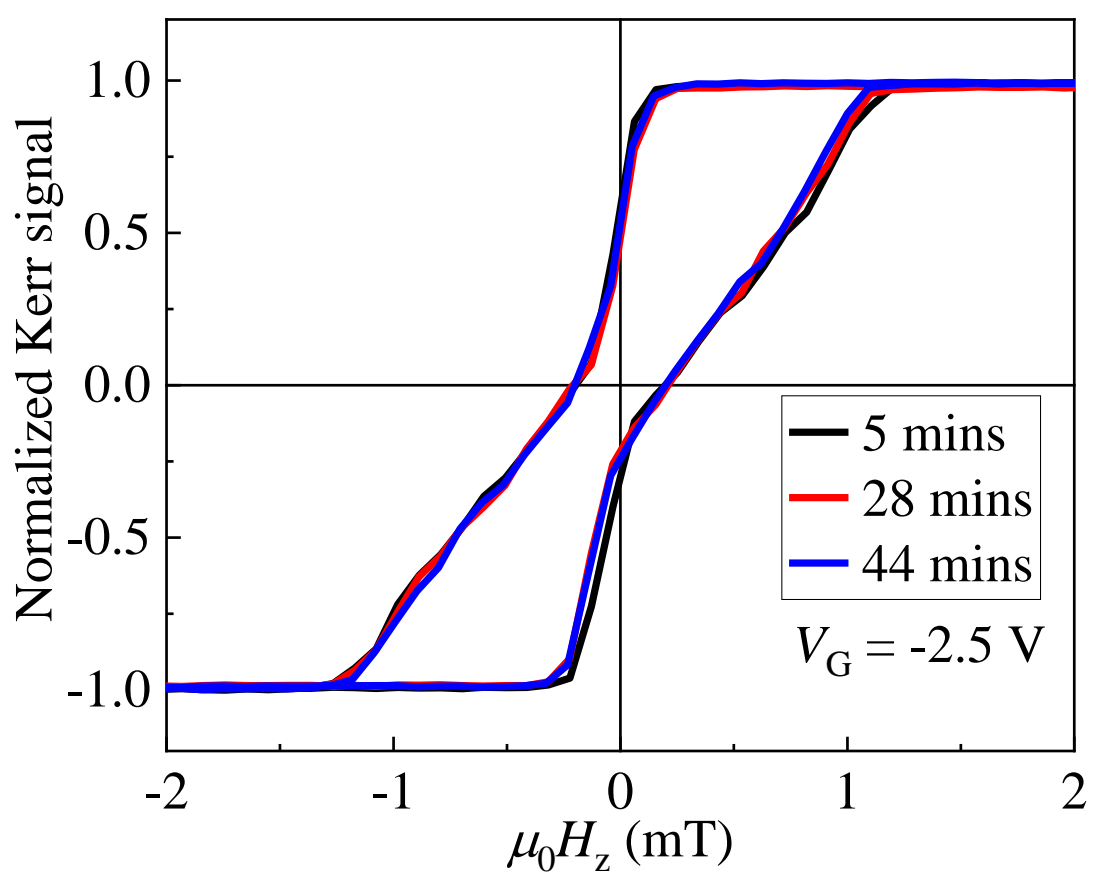

Figure 5.8: The hysteresis loops of $\mathrm{MgO}(1.6 \mathrm{~nm}) / \mathrm{Mn}_{2} \mathrm{CoAl}(2.6 \mathrm{~nm}) / \mathrm{Pd}(3.2 \mathrm{~nm})$ at $-2.5 \mathrm{~V}$ after charging 5,28 , and 44 minutes, respectively.

in direct contact with the pure Pd layer which could induce a significantly large electrostatic charge effect without $\mathrm{PdO}_{x}$ shielding. With an external electric field, Zhao et al., found that the distribution of the charge density in a heavy metal can be varied resulting in a changing of the spin-orbit coupling energy and a Rashba effect in Au/DEME-TFSI/Pt/(Co/Pt $)_{2} / \mathrm{Ta}$ capacitor heterostructure with PMA [92]. For the Rashba effect, the spin degeneracy is removed under an electric field due to the spin-orbit interaction and leads to a effective magnetic field [155]. Therefore, in this film, the magnetic moments rotate from OP to IP direction in a volatile mode at a positive gate voltage due to an in-plane net Rashba magnetic field. By carefully decreasing the magnetic anisotropy, closing to the spinreorientation transition, the skyrmions can be formed in a volatile state. These thin films exhibit an excellent reproducibility of magnetic anisotropy to repeated gate voltages which strongly suggests that, except for dur- 
ing the high voltage trigger, no chemical reaction occurs at low voltage. Magneto-ionic gating effect has been demonstrated in $\mathrm{Pd} / \mathrm{Co} / \mathrm{Pd}$ heterostructures with PMA [153]. In this case, $\mathrm{H}^{+}$ions can be pumped into Pd layer at a positive gate voltage, which induces a $90^{\circ}$ magnetization switching. This gating behaviour is non-volatile, and can be reversed by applying a $-1 \mathrm{~V}$ gate voltage. In the case shown in Figure 5.4, the magnetic anisotropy spontaneously reverses after setting $\mathrm{V}_{\mathrm{G}}$ back to $0 \mathrm{~V}$ suggesting there is not non-volatile magneto-ionic gating effect in this gating method with a trigger.

In summary, the $\mathrm{MgO} / \mathrm{Mn}_{2} \mathrm{CoAl} / \mathrm{Pd}$ ultrathin films were fabricated with different strength of PMA and investigate the skyrmion generation in these thin films without skyrmion at pristine state by ILG. Non-volatile skyrmions can be generated at a negative gate voltage in films with a weak PMA. For the thin films with intermediate PMA, samples can be trained to the skyrmion regime by cycling voltages. The mechanism for both gating methods is that oxygen ions diffuse into the Pd layer and form a $\mathrm{PdO}_{x}$ layer, which induces a significant reduction of effective magnetic anisotropy, with an extremely high anisotropy field tunability. Moreover, volatile skyrmions can be created at a small positive gate voltage after applying a trigger gate voltage to etch away the native oxide $\mathrm{PdO}_{x}$ layer. Without $\mathrm{PdO}_{x}$ shielding, an electrostatic charge accumulation happens at the surface and induces an in-plane net Rashba magnetic field. These results strongly demonstrate that ILG is a powerful and versatile technique towards the generation of skyrmions and skyrmion-based applications. 


\section{Chapter 6}

\section{Large anomalous Hall angle of $\mathrm{Co}_{2} \mathrm{MnGa}$}

\subsection{Introduction}

Skyrmion generation is the firstly crucial step toward skyrmion-based spintronics application. At chapters 4 and 5, the skyrmion creation have investigated in perpendicularly magnetized Heusler alloy $\mathrm{Mn}_{2} \mathrm{CoAl}$ by inplane magnetic field and ionic liquid gating. To realize the real applications, methods are needed to manipulate skyrmions. Spin-polarized current with angular momentum can be used to drive skyrmion motion [6]. Usually, the spin-polarized current can be induced by spin Hall effect [28], in which an electric current passes through a non-magnetic metal, like Pt, $\mathrm{Ta}$, and $\mathrm{W}$, and a transverse pure spin current would be generated at the surface of non-magnetic materials. This pure spin current with angular momentum diffuses into the ferromagnetic layer so that the skyrmions can be driven by spin-orbit torque [50]. Instead of using non-magnetic metal, ferromagnetic materials can also induce an anomalous spin-orbit torque (ASOT) due to the anomalous Hall effect [156].

In order to obtain a large and efficient ASOT, materials with a large anomalous Hall angle (AHA) is needed. The AHA is defined by the ra- 
tio of the Hall and longitudinal conductivities, $\theta_{\mathrm{H}}=\sigma_{\mathrm{xy}} / \sigma_{\mathrm{xx}}$. The full Heusler alloy $\mathrm{Co}_{2} \mathrm{MnGa}$ is a ferromagnetic Weyl semimetal as mentioned in subchapter1.7.4. It shows a large Curie temperature $T_{\mathrm{c}} \approx 700 \mathrm{~K}$ and a large AHA, $12 \%[79,78]$, at room temperature, due to a large net Berry curvature near the Fermi energy. To realize the ASOT for spintronics applications, $\mathrm{CO}_{2} \mathrm{MnGa}$ needs to be fabricated as thin films. Thus, it is important to know if the AHA is still robust at low dimension.

However, almost all of this work was done before the topological characteristics of this material were realized [157, 158, 159, 160]. Hence, there has so far been very little research about the transport properties related to the topological band structure in $\mathrm{Co}_{2} \mathrm{MnGa}$ thin films. Reichlova et al. found that $\mathrm{Co}_{2} \mathrm{MnGa}$ thin films exhibit a large anomalous Nernst effect coefficient at $300 \mathrm{~K},-2$ to $-3 \mu \mathrm{V} \mathrm{K}^{-1}$, due to the non-trivial band structure [161, 162]. However, this coefficient decreases significantly with thickness down to $10 \mathrm{~nm}$ and the reason still needs to be uncovered. Very recently, Markou and Wang et al. investigated thick $\mathrm{Co}_{2} \mathrm{MnGa}$ thin films, which show the same value of anomalous Hall angle as in the single crystals $[163,164]$, but they did not address the reason for the decline of anomalous Hall angle with decreasing thickness. A comprehensive understanding of Weyl topological effects on the AHE in magnetic WSM thin films is important for understanding the prospect of producing energy-efficient, high-density spintronic devices, as well as investigating the potential effects of their low dimensionality on the Berry curvature.

In this chapter, the transport properties of $\mathrm{Co}_{2} \mathrm{MnGa}$ thin films with thicknesses from $50 \mathrm{~nm}$ down to $5 \mathrm{~nm}$ were systematically investigated. The thicker films exhibit a large AHA which can be ascribed to the intrinsic Berry curvature. However, for films thinner than $15 \mathrm{~nm}$, the AHA drops significantly due to the reduction of the majority spin contribution to the Berry curvature, proving that tuning the Berry curvature can be achieved in this Heusler alloy. These results suggest that $\mathrm{Co}_{2} \mathrm{MnGa}$ can be a great potential spin torque generator for driving skyrmions motion. 


\subsection{Results}

\subsubsection{Sample preparation}

$\mathrm{Co}_{2} \mathrm{MnGa}(5-50 \mathrm{~nm})$ thin films are epitaxially deposited on single-crystalline $\mathrm{MgO}(001)$ substrates. Before fabricating thin films, substrates are cleaned with an Ar plasma, and then annealed at $400{ }^{\circ} \mathrm{C}$ for $1 \mathrm{~h}$ in the vacuum chamber. $\mathrm{Co}_{2} \mathrm{MnGa}$ thin films are DC magnetron sputtered from a stoichiometric polycrystalline target at $100 \mathrm{~W}$ under 6 mTorr of Ar with a growth rate of $0.8 \AA \mathrm{s} \mathrm{s}^{-1}$ at $400{ }^{\circ} \mathrm{C}$ while rotating the sample holder. All samples are post-annealed in situ at $550{ }^{\circ} \mathrm{C}$ for $1 \mathrm{~h}$ to improve the lattice structure and the atomic ordering among $\mathrm{Co}, \mathrm{Mn}$, and Ga sites. After cooling down to ambient temperature, a $2 \mathrm{~nm}$ protective $\mathrm{MgO}$ layer is deposited on the top. The elemental composition of $\mathrm{Co}_{2} \mathrm{MnGa}$ was calibrated by EDX, as shown in Figure 2.9(b). A good stoichiometry, $\mathrm{Co}_{1.96} \mathrm{Mn}_{0.96} \mathrm{Ga}_{1.08}$, can be obtained. Samples are patterned into standard Hall bars $(l=2000 \mu \mathrm{m}$, $w=150 \mu \mathrm{m})$ for magnetotransport characterization.

\subsubsection{Structure Characterization}

For the $50 \mathrm{~nm}$ film, the $\theta-2 \theta$ scan of the XRD pattern shows the substrate $\mathrm{MgO}(002)$ peak and also $\mathrm{Co}_{2} \mathrm{MnGa}(002)$ and (004) peaks, as shown in Figure 6.1(a), indicating a good out of plane texture. Other $\mathrm{MgO}[00 \mathrm{l}]$ lines are indicated by an asterisk, $*$. The inset is the rocking curve from the (004) peak, and the full width at half maximum (FWHM) is $0.779^{\circ}$ suggesting a high crystal quality. Furthermore, the X-ray $\varphi$ scans from $-45^{\circ}$ to $315^{\circ}$ (Figure 6.1(b)) display a fourfold symmetry of the $\mathrm{Co}_{2} \mathrm{MnGa}(220)$ peak and the $45^{\circ}$ rotation of these peaks relative to the substrate (220) peaks, confirming the epitaxial relationship of $\mathrm{MgO}(001)[100] \| \mathrm{Co}_{2} \mathrm{MnGa}(001)[110]$. The superstructure (111) peak with in-plane XRD was also obtained, which shows fourfold symmetry in the $\varphi$ scan as well, as shown in the top panel of Figure 6.1(b). 
In full Heusler alloys, the XRD peaks with all odd $(h k l)$ indexes, like (111), are known to originate in superlattice reflections in the $L 2_{1}$ structure [57]. The in-plane $a-b$ and $c$-axis lattice constants are $5.765 \AA$ and $5.759 \AA$ $(c / a=0.999)$, respectively, obtained from the (004) and (111) peaks, indicating that the crystal structure is very close to the cubic Heusler structure, with only a small tetragonal distortion. The strain $\varepsilon=\left(a_{/ /}-a_{0}\right) / a_{0}$ is estimated as $-0.035 \%$, where $a_{0}$ is the lattice constant of bulk single crystal $\mathrm{Co}_{2} \mathrm{MnGa}(5.767 \AA)$ [165] and $a_{\|}$is the in-plane lattice constant of thin film $(5.765 \AA)$. Even though the diagonal $\sqrt{2 a} \mathrm{MgO}$ of the substrate is $5.962 \AA$, which could induce a $3.27 \%$ lattice mismatch, the stress in these thin film is almost fully relaxed due to the post-annealing at $550{ }^{\circ} \mathrm{C}$ for $1 \mathrm{~h}$.

The film roughnesses measured by XRR or AFM are below $1 \mathrm{~nm}$, as shown by example for the $20 \mathrm{~nm}$ thick film in Figure 6.1(c). Fitting the XRR results in a root-mean-square roughness $\left(R_{\mathrm{q}}\right)$ of $0.7 \mathrm{~nm}$, with the thickness $20 \mathrm{~nm} \pm 2 \mathrm{~nm}$. The AFM image confirms a smooth surface morphology with a $R_{\mathrm{q}}$ of $0.5 \mathrm{~nm}$, as shown in the insert of Figure 6.1(c).

\subsubsection{Magnetotransport properties}

Magnetic hysteresis loops of a $50 \mathrm{~nm} \mathrm{Co}_{2} \mathrm{MnGa}$ film are measured with the external magnetic field along both the in-plane and out-of-plane directions at $300 \mathrm{~K}$ by the SQUID magnetometer, as shown in Figure 6.2(a). These show the easy axis is along the $\mathrm{Co}_{2} \mathrm{MnGa}[110]$ direction, the same as for other Heusler alloy thin films, like $\mathrm{Co}_{2} \mathrm{MnAl}$ and $\mathrm{Co}_{2} \mathrm{MnSi}[166,167]$, and the coercive field $\mu_{0} H_{\mathrm{c}}$ and saturation magnetization $M_{\mathrm{s}}$ are $3 \mathrm{mT}$, $6 \times 10^{5} \mathrm{~A} / \mathrm{m}$, respectively. Note that the crystalline direction used in this chapter refers to the $\mathrm{Co}_{2} \mathrm{MnGa}$ lattice, unless indicated otherwise. When the magnetic field is along the [100] direction, though it shows a small coercive field $\left(\mu_{0} H_{\mathrm{c}}=1 \mathrm{mT}\right.$ ), the saturation field is $25 \mathrm{mT}$, larger than along the [110] direction. For the [001] direction, the saturation field is $1 \mathrm{~T}$, but it still shows a small square loop, interestingly indicating there is a small 

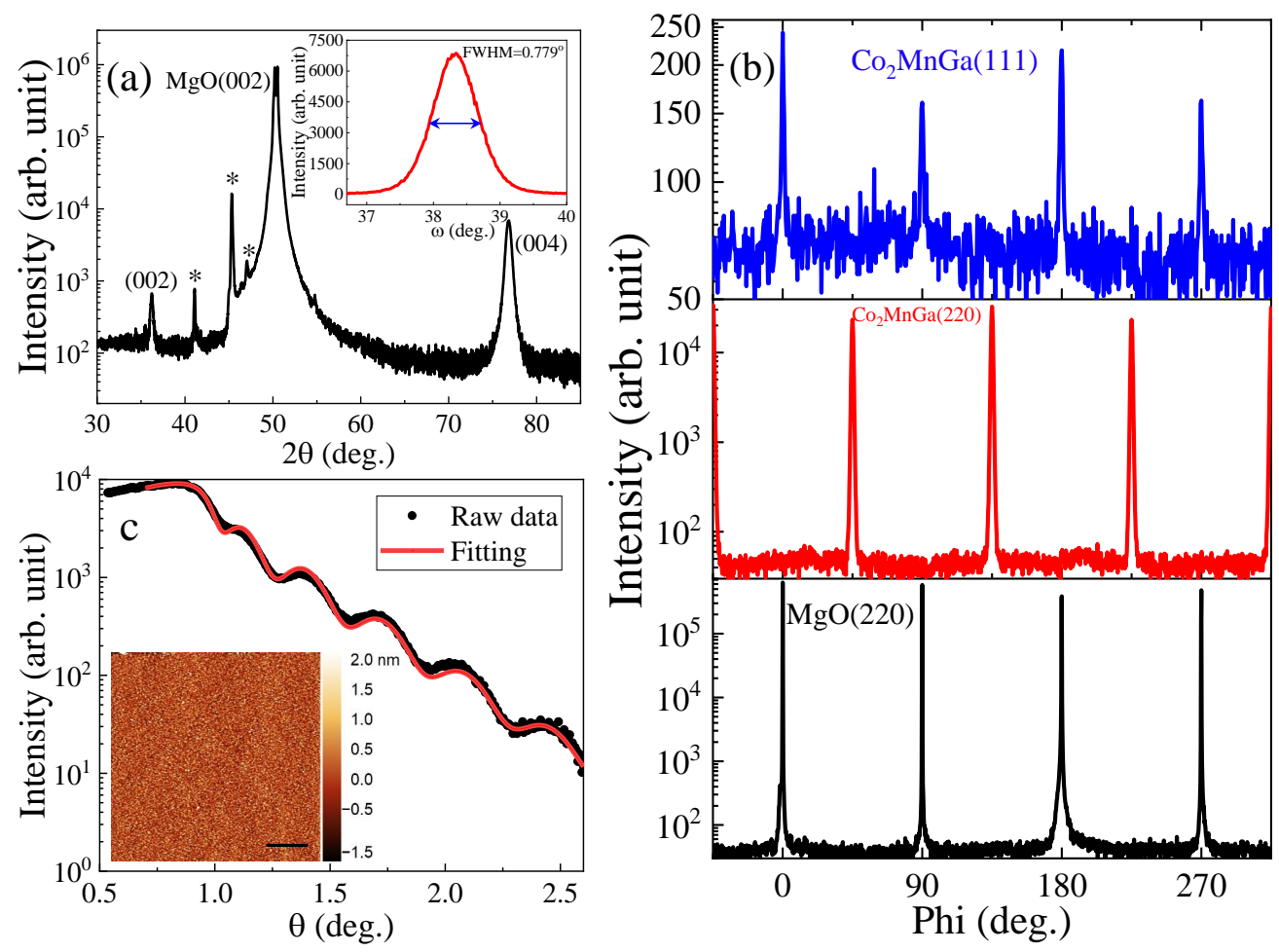

Figure 6.1: Crystal structure and surface morphology of $\mathrm{Co}_{2} \mathrm{MnGa}$. (a) $\theta-2 \theta$ scan, the inset is the rocking curve from (004) peak, and (b) in-plane $\varphi$ scans from $-45^{\circ}$ to $315^{\circ}$, for $50 \mathrm{~nm} \mathrm{Co}{ }_{2} \mathrm{MnGa}$ thin films. (c) X-ray reflectivity for the $20 \mathrm{~nm}$ $\mathrm{Co}_{2} \mathrm{MnGa}$ thin film, the inset is the atomic force microscopy image $\left(5 \times 5 \mu \mathrm{m}^{2}\right)$ with colour contrast (dark to bright) for the height scale corresponds to $3.5 \mathrm{~nm}$. The scale bar corresponds to $1 \mu \mathrm{m}$.

out-of-plane magnetization component at zero applied field. The uniaxial anisotropy energy density calculated from the hard [001] and easy [110] directions is $3.05 \times 10^{5} \mathrm{~J} \mathrm{~m}^{-3}$.

The Hall resistivity as a function of magnetic field is measured at $300 \mathrm{~K}$ and $3 \mathrm{~K}$, respectively, as shown in Figure 6.2(b). For all of these samples, the current is applied along the [100] direction. The pure anomalous Hall resistivity is calculated by using

$$
\rho_{\mathrm{AH}}=\rho_{\mathrm{xy}}-R_{\mathrm{o}} H_{\mathrm{z}},
$$



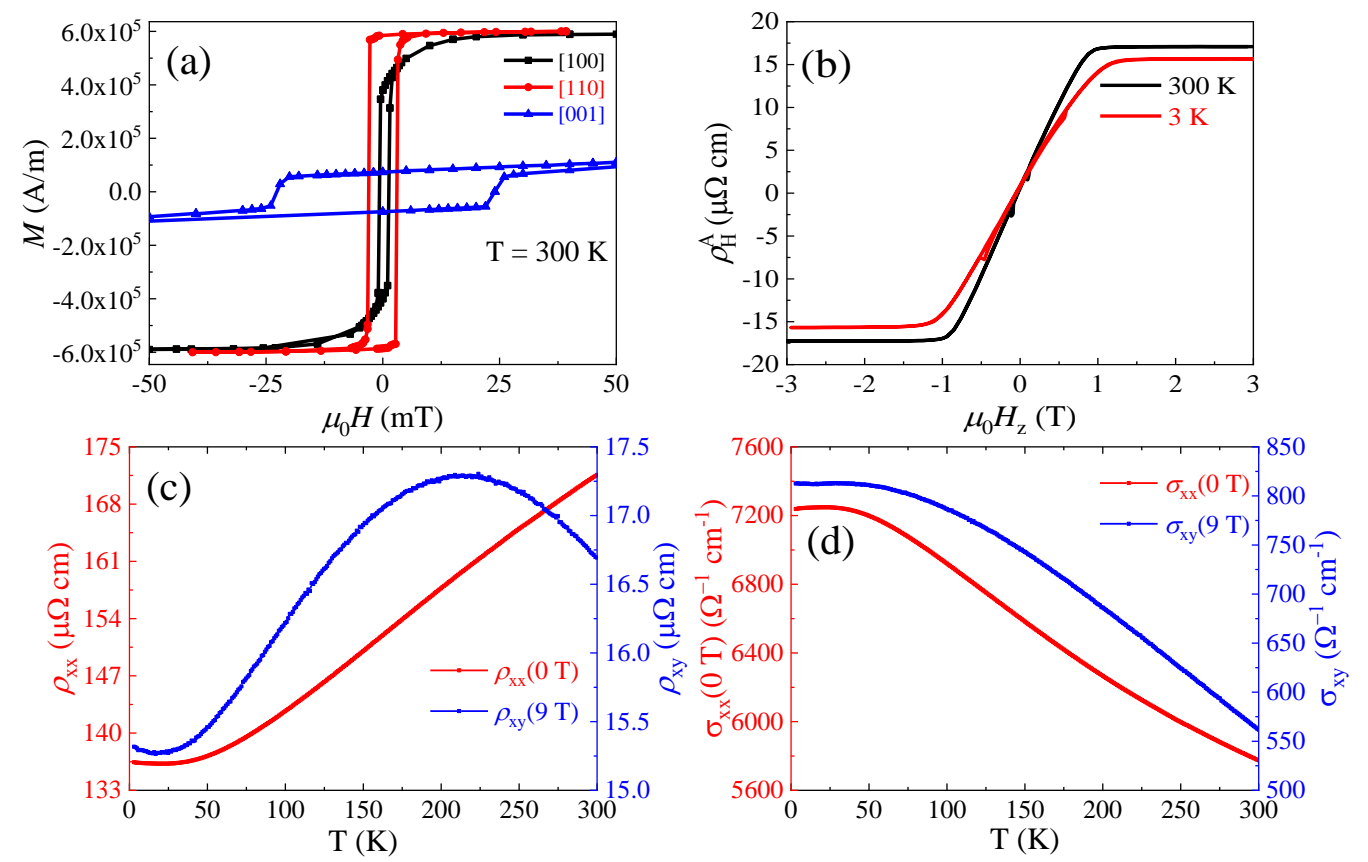

Figure 6.2: Magnetic and electric transport measurements for $50 \mathrm{~nm} \mathrm{Co}{ }_{2} \mathrm{MnGa}$. (a) Magnetization measured at $300 \mathrm{~K}$ with the magnetic field along in-plane and out-of-plane direction, respectively. (b) Anomalous Hall effect measurements at 3 and $300 \mathrm{~K}$, respectively. (c) Longitudinal resistivity without field (red) and Hall resistivity cooling down with a $9 \mathrm{~T}$ magnetic field (blue) as a function of temperature from 3 to $300 \mathrm{~K}$. (d) The corresponding conductivity as a function of temperature from 3 to $300 \mathrm{~K}$.

where $R_{\mathrm{o}}$ is the ordinary Hall coefficient and $H_{\mathrm{z}}$ is the magnetic field along out-of-plane direction. The Hall coefficient $R_{\mathrm{o}}$ is negative and quite small, $2.42 \times 10^{-4} \mathrm{~cm}^{3} \mathrm{C}^{-1}(300 \mathrm{~K})$ and $1.06 \times 10^{-4} \mathrm{~cm}^{3} \mathrm{C}^{-1}(3 \mathrm{~K})$, meaning that charge carriers are of the electron type. The anomalous Hall saturation fields at 300 and $3 \mathrm{~K}$ are 1.3 and $1.6 \mathrm{~T}$, respectively. The anomalous Hall resistivities are 17.1 and $15.4 \mu \Omega \mathrm{cm}$ at 300 and $3 \mathrm{~K}$, respectively, and the ordinary Hall resistivities at $9 \mathrm{~T}$ are $0.218 \mu \Omega \mathrm{cm}(300 \mathrm{~K})$ and $0.095 \mu \Omega \mathrm{cm}$ $(3 \mathrm{~K})$. The Hall carrier concentration and Hall mobility are $2.58 \times 10^{22} \mathrm{~cm}^{-3}$ and $1.41 \mathrm{~cm}^{2} \mathrm{~V}^{-1} \mathrm{~s}^{-1}$, respectively, at $300 \mathrm{~K}$. Since the ordinary Hall resis- 
tivity is negligible in these samples, it is safe to adopt $\rho_{\mathrm{xy}}$ as nearly equal to the anomalous Hall resistivity. The knot in the Hall resistivity $v s \mu_{0} H_{z}$ curve also indicates the out-of-plane magnetization component seen in the magnetization measurements.

In Figure 6.2(c), the temperature dependence of Hall resistivity $\rho_{\mathrm{xy}}$ and longitudinal resistivity $\rho_{\mathrm{xx}}$ obtained from 300 to $3 \mathrm{~K}$ are plotted. The $50 \mathrm{~nm}$ $\mathrm{Co}_{2} \mathrm{MnGa}$ thin film has a residual resistivity $\rho_{\mathrm{o}}=136 \mu \Omega \mathrm{cm}$ at $23 \mathrm{~K}$ and exhibits a typical metallic behaviour at higher temperature (red curve). The $\rho_{\mathrm{xy}}$ increases with $\mathrm{T}$, displaying a maximum value $17 \mu \Omega \mathrm{cm}$ around $210 \mathrm{~K}$, followed by a decrease to higher temperature (blue curve). The Hall conductivity $\sigma_{\mathrm{xy}}$ and longitudinal conductivity $\sigma_{\mathrm{xx}}$ are calculated by using $\sigma_{\mathrm{xy}}=\rho_{\mathrm{xy}} /\left(\rho_{\mathrm{xy}}^{2}+\rho_{\mathrm{xx}}^{2}\right)(0 T)$ and $\sigma_{\mathrm{xx}}=\rho_{\mathrm{xx}} /\left(\rho_{\mathrm{xy}}^{2}+\rho_{\mathrm{xx}}^{2}\right)(0 T)$, respectively. Both Hall conductivity (blue curve) and longitudinal conductivity (red curve) increase monotonically on cooling and approach constant values of 812 and $7250 \Omega^{-1} \mathrm{~cm}^{-1}$, respectively, at low temperature, as shown in Figure 6.2(d).
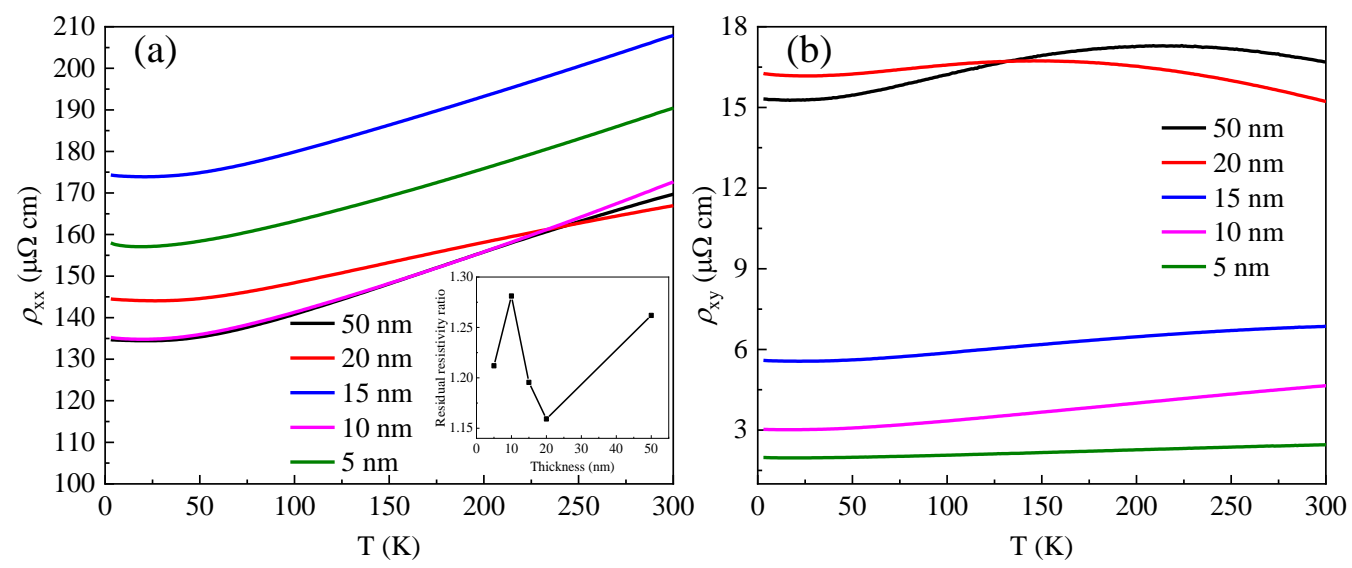

Figure 6.3: (a) Longitudinal resistivity without field as a function of temperature from $3 \mathrm{~K}$ to $300 \mathrm{~K}$ for $\mathrm{Co}_{2} \mathrm{MnGa}(5-50 \mathrm{~nm})$. The inset shows the residual resistivity ratio $\rho_{\mathrm{xx}}(300 \mathrm{~K}) / \rho_{\mathrm{xx}}$ (residual) as a function of thickness. (b) Anomalous Hall resistivity cooling down in $9 \mathrm{~T}$ as a function of temperature from $3 \mathrm{~K}$ to $300 \mathrm{~K}$ for $\mathrm{Co}_{2} \mathrm{MnGa}(5-50 \mathrm{~nm})$. 
To understand the thickness dependence of the magnetic transport properties, $\mathrm{Co}_{2} \mathrm{MnGa}$ films with smaller thicknesses, from 20 to $5 \mathrm{~nm}$, were fabricated. Figure 6.3 shows the $\rho_{\mathrm{xx}}$ and $\rho_{\mathrm{xy}}$ as a function of temperature from $3 \mathrm{~K}$ to $300 \mathrm{~K}$ for $\mathrm{Co}_{2} \mathrm{MnGa}(5-50 \mathrm{~nm})$. The $\rho_{\mathrm{xx}}$ is in the range of 167 to $208 \mu \Omega \mathrm{cm}$ at $300 \mathrm{~K}$ for $\mathrm{Co}_{2} \mathrm{MnGa}$, which is similar to other group's results [164, 163]. Especially of note, the $\rho_{\mathrm{xx}}$ of $10 \mathrm{~nm} \mathrm{Co}_{2} \mathrm{MnGa}$ films is almost same as one of $50 \mathrm{~nm}$. This small difference suggests that all films show similar crystalline quality since films with different order (B2) or disordered films show higher resistivity. What's more, the residual resistivity ratios (see inset of Figure 6.3(a) of all $\mathrm{Co}_{2} \mathrm{MnGa}$ films in these results are similar and even slightly larger than films from previous reports in which a good $L 2_{1}$-order structure has been confirmed (e.g. $30 \mathrm{~nm}$ film in [164] and $80 \mathrm{~nm}$ in [163].

The AHA can be calculated by using $\theta_{\mathrm{H}}=\sigma_{\mathrm{xy}} / \sigma_{\mathrm{xx}}$. Figure 6.4 shows the AHA as a function of temperature from 300 to $3 \mathrm{~K}$. The $50-\mathrm{nm}$ ferromagnetic Weyl semimetal $\mathrm{Co}_{2} \mathrm{MnGa}$ thin film shows a large AHA $\theta_{\mathrm{H}}=$ $9.7 \%$ at $300 \mathrm{~K}$, increasing at low temperature to reach a maximum value $11.4 \%$ at $113 \mathrm{~K}$. Usually, typical ferromagnetic thin films with topologically trivial band structure show a small angle, less than 3\% [97]. Even though some non-magnetic Weyl semimetals exhibit giant AHA in single crystals $[168,169]$, to date only $\mathrm{Co}_{2} \mathrm{MnG}$ a shows such a large AHA in thin films at room temperature.

Figure 6.4(b) shows the thickness dependence of the AHA measured from 300 to $3 \mathrm{~K}$. Once the thickness is decreased to $20 \mathrm{~nm}$, the AHA is still large, around $11 \%$, and this film shows a similar trend as the $50-\mathrm{nm}$ film when decreasing the temperature. However, for samples with thicknesses of 15,10 , and $5 \mathrm{~nm}$, the AHA is temperature independent, and the value drops to $3.3 \%, 2.7 \%$, and $1.3 \%$, respectively. The inset shows the maximum value of each sample, as measured between 3 and $300 \mathrm{~K}$. These results of thinner samples agree very well with ref.[170] in which the AHA of $6 \mathrm{~nm}$ $\mathrm{Co}_{2} \mathrm{MnGa}$ is no more than $2.8 \%$. Figure $6.4(\mathrm{c})$ is the $\log \sigma_{\mathrm{xy}}-\sigma_{\mathrm{xx}}$ plot for 
all samples measured at 300 to $3 \mathrm{~K} . \sigma_{\mathrm{xx}}$ is about $4.5-7.5 \times 10^{3} \Omega^{-1} \mathrm{~cm}^{-1}$ at various thicknesses and temperatures, and $\sigma_{\mathrm{xy}}$ is nearly independent of $\sigma_{\mathrm{xx}}$. For the samples with thicknesses from 50 to $20 \mathrm{~nm}$, one can see that $\sigma_{\mathrm{xy}}$ is nearly constant (around $700 \Omega^{-1} \mathrm{~cm}^{-1}$ ).

It is known that there exist three regimes in the scaling behaviour of the AHE depending on the range of $\sigma_{\mathrm{xx}}$, categorized by a scaling parameter, $\alpha$, in $\sigma_{\mathrm{xy}} \propto \sigma_{\mathrm{xy}}^{\alpha}$ [96]. The values of $\sigma_{\mathrm{xx}}$ of all samples are close to the intermediate region as defined in ref.[96], where $\sigma_{\mathrm{xx}}=10^{4}-10^{6} \Omega^{-1} \mathrm{~cm}^{-1}$. Furthermore, this constant value of $\sigma_{\mathrm{xy}}$ is close to the intrinsic contribution (around $1000 \Omega^{-1} \mathrm{~cm}^{-1}$ ). This is the value expected from the intrinsic scattering-independent mechanism originating from the Berry curvature in momentum space, and indicates that $\sigma_{\mathrm{xy}}$ in these films is dominated by the intrinsic component due to the non-trivial topology of band structure of $\mathrm{Co}_{2} \mathrm{MnGa}$ [97]. However, for the thinner samples with thicknesses of 15 , 10 , and $5 \mathrm{~nm}$, the values of $\sigma_{\mathrm{xy}}$ drop steeply to 170,160 , and $70 \Omega^{-1} \mathrm{~cm}^{-1}$, respectively. Since the values of $\sigma_{\mathrm{xy}}$ of these samples are much smaller than the intrinsic contribution, $\sigma_{\mathrm{xy}}$ might be due not only to the intrinsic scattering, but also might include contributions from the extrinsic side-jump mechanism, which can also induce values of $\sigma_{\mathrm{xy}}$ that are independent of $\sigma_{\mathrm{xy}}$ [171]. This agrees with results in ref.[134] in which $\sigma_{\mathrm{xy}}$ of polycrystalline $\mathrm{MgO} / \mathrm{Co}_{2} \mathrm{MnGa} / \mathrm{Pd}$ ultrathin films results from both intrinsic and side-jump scattering.

Figures 6.4(d) and (e) show $\sigma_{\mathrm{xx}}$ and $\sigma_{\mathrm{xy}}$ against the temperature and thickness, respectively. $\sigma_{\mathrm{xx}}$ slightly increases with decreasing temperature, and then remains constant at low temperature. $\sigma_{\mathrm{xx}}$ for all samples at various temperatures varies only by $25 \%$; however, $\sigma_{\mathrm{xy}}$ drops suddenly once the thickness is below $15 \mathrm{~nm}$. For instance, comparing the $50 \mathrm{~nm}$ and 10 nm films, $\sigma_{\mathrm{xx}}$ of both is around $7.2 \times 10^{3} \Omega^{-1} \mathrm{~cm}^{-1}$ at $3 \mathrm{~K}$, but $\sigma_{\mathrm{xy}}$ drops from 810 to $160 \Omega^{-1} \mathrm{~cm}^{-1}$ for the thinner film.

In non-magnetic WSMs, the existence of planar Hall effects or negative magnetoresistances has also been taken as further evidence of the effect of 

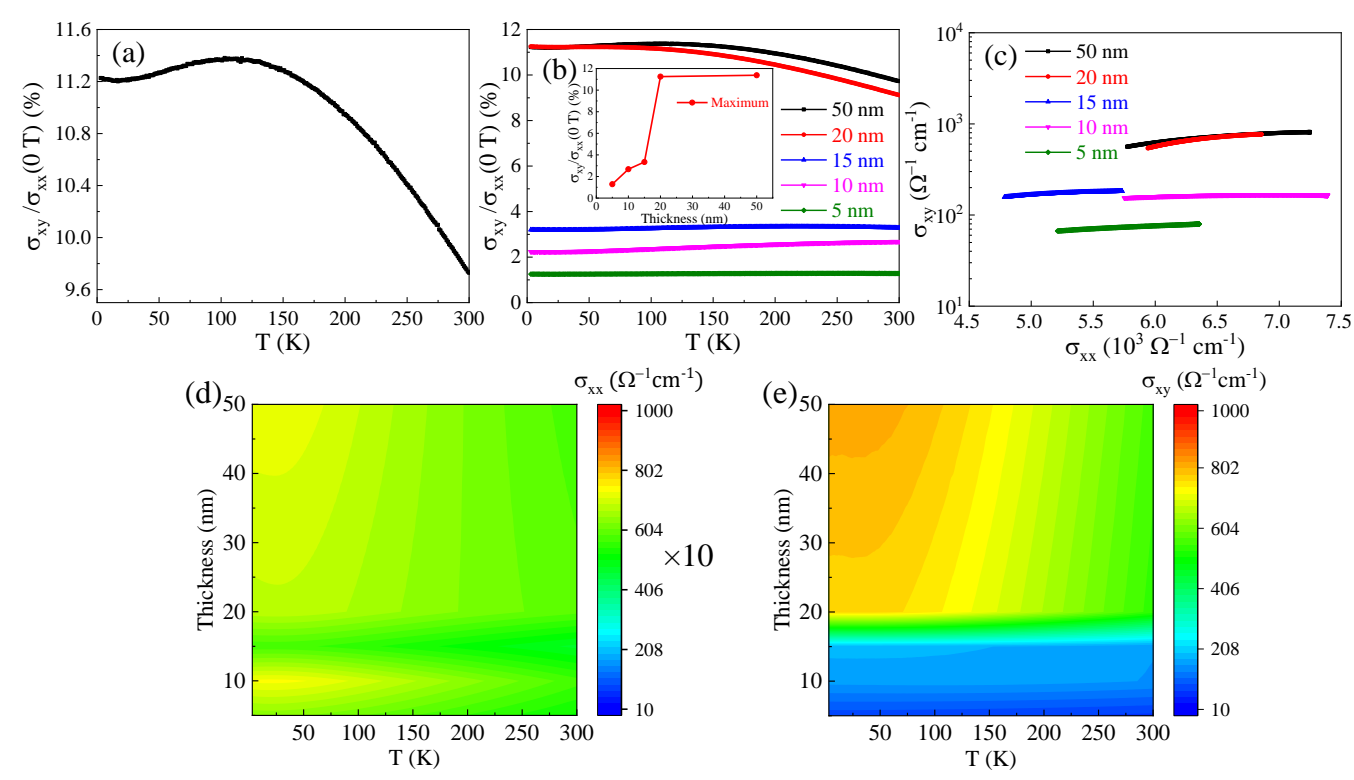

Figure 6.4: Transport measurement of the AHA at $9 \mathrm{~T}$. (a) Temperature dependent AHA measured from $3 \mathrm{~K}$ to $300 \mathrm{~K}$ for $50 \mathrm{~nm} \mathrm{Co} 2 \mathrm{MnGa}$. (b) Thickness dependence of AHA as a function of temperature from $3 \mathrm{~K}$ to $300 \mathrm{~K}$, the inset is the maximum AHA as a function of thickness. (c) $\log \sigma_{\mathrm{xy}}-\sigma_{\mathrm{xx}}$ plot for $\mathrm{Co}_{2} \mathrm{MnGa}$ with thicknesses from $5 \mathrm{~nm}$ to $50 \mathrm{~nm}$. (d)-(e) Contour mapping for thickness dependence of $\sigma_{\mathrm{xx}}$ and $\sigma_{\mathrm{xy}}$ as a function of temperature from $3 \mathrm{~K}$ to $300 \mathrm{~K}$, respectively.

the Weyl character of the band structure on the electronic transport [172, 173]. However, both of these effects are present in magnetic non-Weyl materials also, and the measurements of these effects in magnetic WSM $\mathrm{Co}_{3} \mathrm{Sn}_{2} \mathrm{~S}_{2}$ are unremarkable and consistent with a trivial magnetic origin [174].

In Figures 6.5 and 6.6 the angle-dependent planar Hall effect and magnetoresistance of $\mathrm{Co}_{2} \mathrm{MnGa}$ films measured along several crystallographic directions. The magnitudes of these effects are similar to those measured in $\mathrm{Co}_{3} \mathrm{Sn}_{2} \mathrm{~S}_{2}[174,11]$ and are not larger than in typical magnetic non-Weyl materials. The linear negative MR observed at high field in $\mathrm{Co}_{2} \mathrm{MnGa}$ when the magnetic field is parallel to the current, as shown in Figure 6.6, 

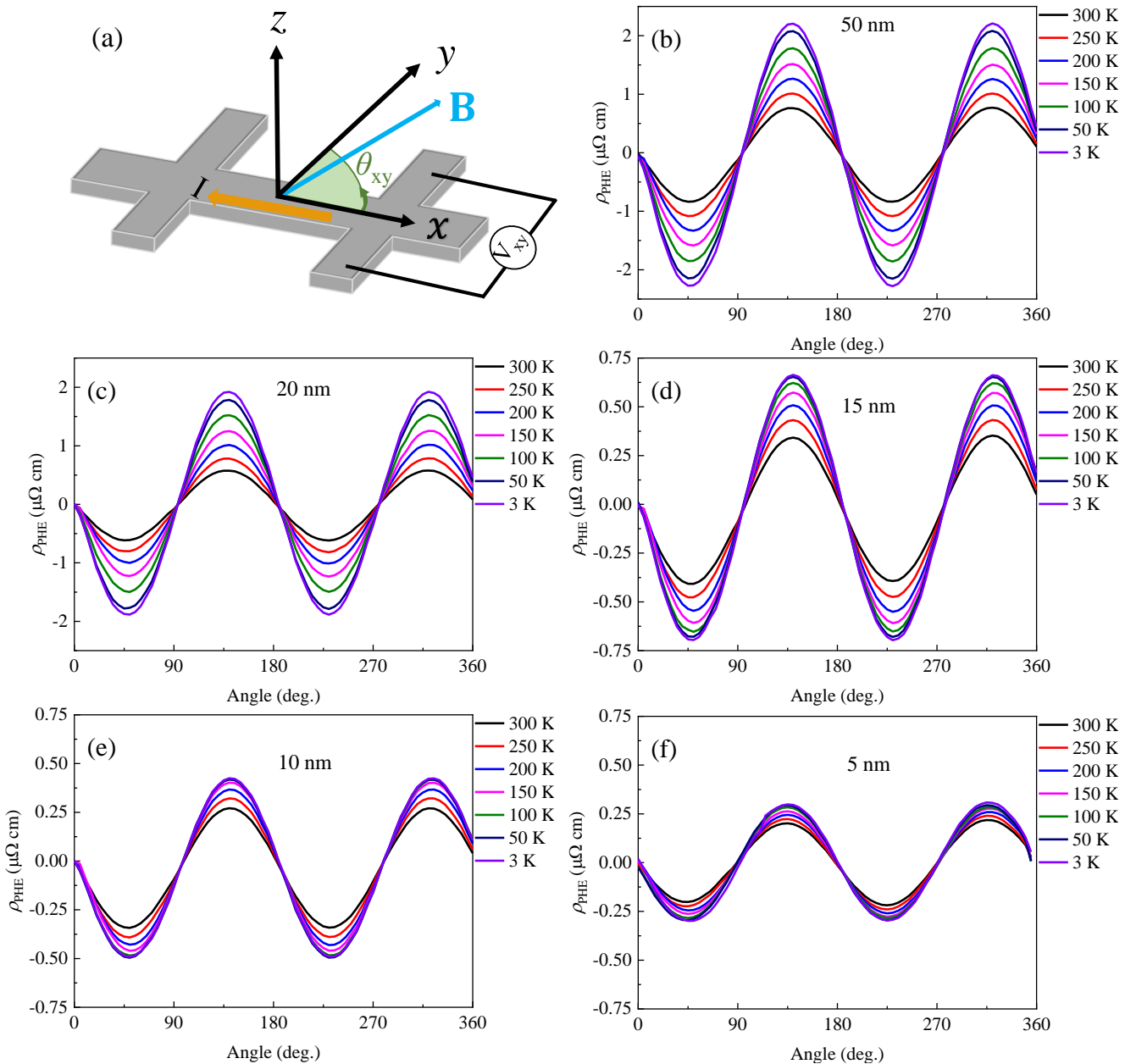

Figure 6.5: Angular dependence of planar Hall resistivity for various thickness of $\mathrm{Co}_{2} \mathrm{MnGa}$ film. (a) Schematic of measurement geometry. The angle $\theta_{\mathrm{xy}}$ represents the angle between the rotating field and $x$-axis when the field rotates from $x$-axis to $y$-axis in the $x y$ plane. Current is along the [100] direction of $\mathrm{Co}_{2} \mathrm{MnGa}$. (b)(f) Planar Hall resistivity as a function of angle, $\theta_{\mathrm{xy}}$, measured from $300 \mathrm{~K}$ and $3 \mathrm{~K}$ under a magnetic field of $9 \mathrm{~T}$ for 50, 20, 15, 10 and $5 \mathrm{~nm} \mathrm{Co} \mathrm{Cn}_{2} \mathrm{Ma}$ film, respectively.

can be ascribed to the thermal-induced spin disorder, which is a typical ferromagnet behaviour $[175,170]$. Therefore, the large AHA might be the only consistent magnetotransport indicator of the topological non-trivial 

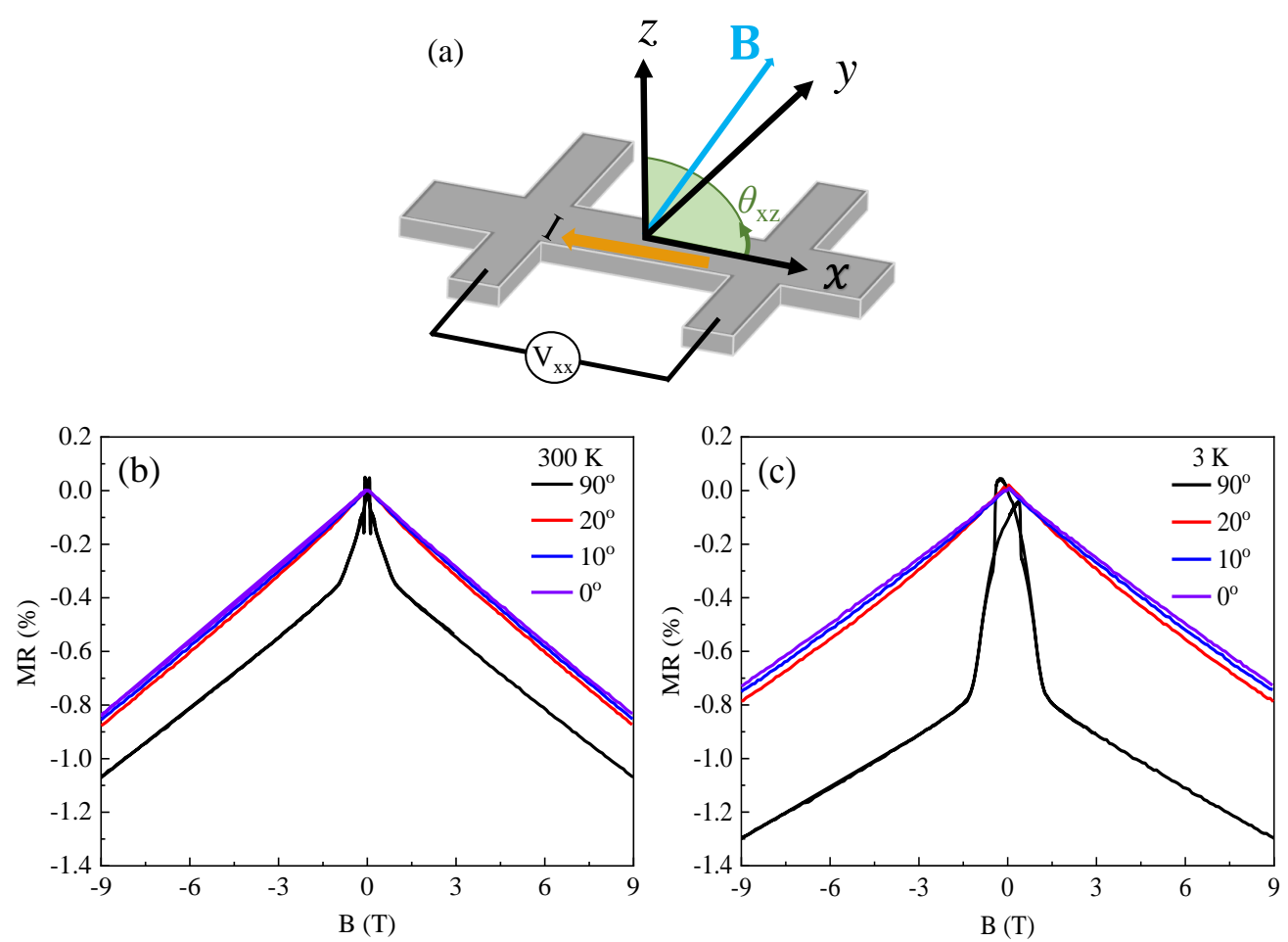

Figure 6.6: Magnetoresistance (MR) ratio of the $50 \mathrm{~nm} \mathrm{Co} \mathrm{Cn}_{2} \mathrm{MnG}$ film. (a) Schematic of measurement geometry. The angle $\theta_{\mathrm{xz}}$ represents the angle between the rotating field and $x$-axis when the field rotates from $x$-axis to $z$-axis in the $x z$ plane. Current is along the [100] direction of $\mathrm{Co}_{2} \mathrm{MnGa}$. (b) and (c) MR ratio as a function of magnetic field measured at $300 \mathrm{~K}$ and $3 \mathrm{~K}$ with various angles, $\theta_{\mathrm{xz}}$, respectively.

band structure in ferromagnetic WSMs such as $\mathrm{Co}_{2} \mathrm{MnGa}$.

\subsubsection{Evolution of Berry curvature and surface band struc- ture}

The intrinsic contribution to $\sigma_{\mathrm{xy}}$ is dependent only on the band structure of a material. It can be calculated from the Kubo formula for the Hall conductivity 

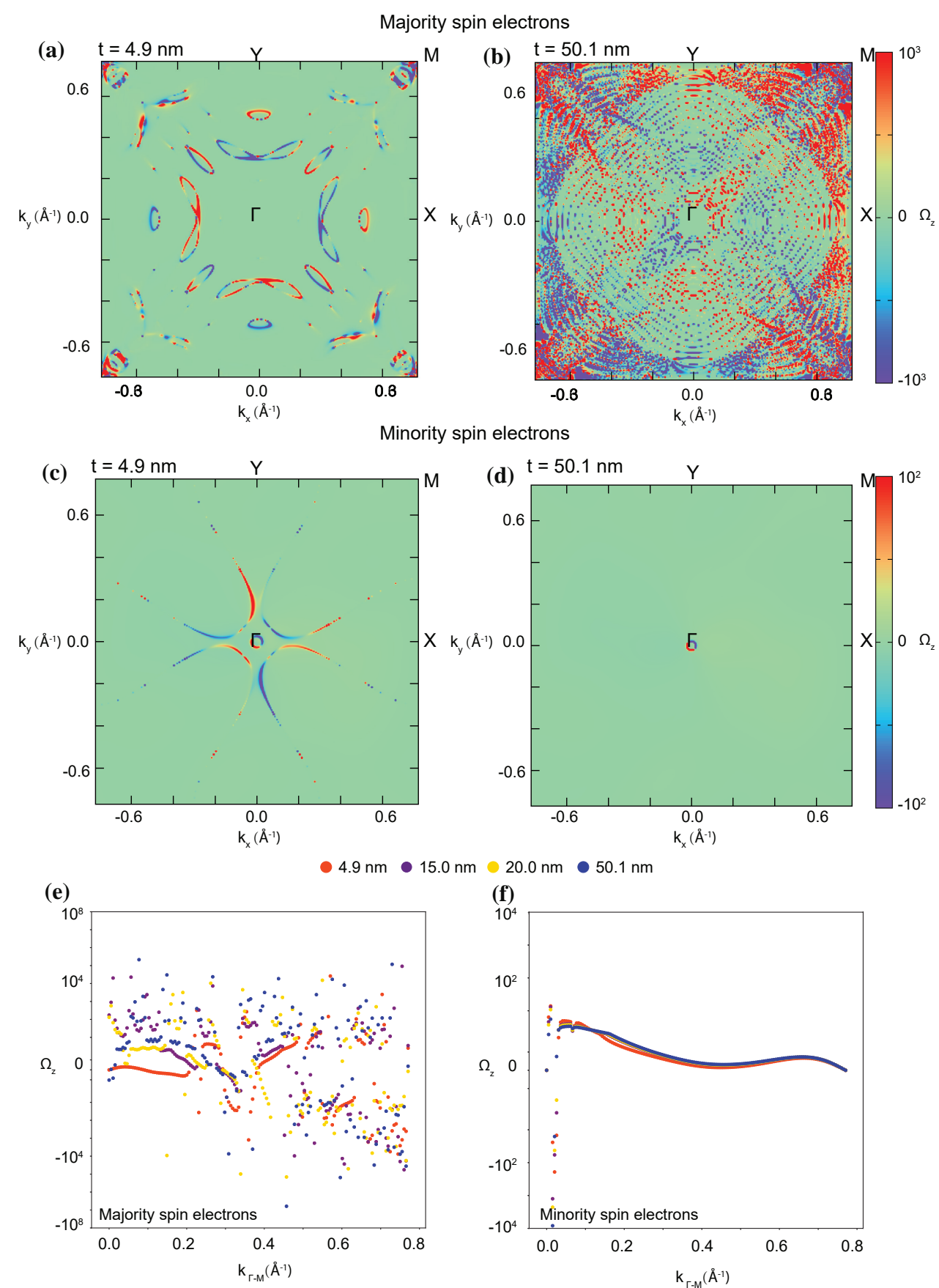

Figure 6.7: (a)-(d) show the calculated averaged-integral of $z$ component Berry curvature $\left(\Omega_{\mathrm{z}}\right)$ of occupied bands from minority and majority spin electrons for $\mathrm{Co}_{2} \mathrm{MnGa}$ thin films of $4.9 \mathrm{~nm}$ and $50.1 \mathrm{~nm}$. (e) and (f) show a comparison of $\Omega_{\mathrm{z}}$ along $\Gamma$-M line for $\mathrm{Co}_{2} \mathrm{MnGa}$ thin films of 4.9, 15.0, 20.0, and $50.1 \mathrm{~nm}$. 


$$
\sigma_{\mathrm{xy}}=\frac{\mathrm{e}^{2}}{\hbar} \int \frac{d \boldsymbol{k}}{2 \pi^{3}} f\left(\varepsilon_{k}\right) \Omega_{\mathrm{z}}
$$

where $f\left(\varepsilon_{k}\right)$ is the Fermi-Dirac distribution function, $\Omega_{\mathrm{z}}$ is the $z$ component Berry curvature [176]. $\sigma_{\mathrm{xy}}$ can also be found from the sum of the conductivities for majority spin electrons $(\uparrow)$ and minority spin electrons $(\downarrow), \sigma_{\mathrm{xy}}=\sigma_{\mathrm{xy}}^{\uparrow}+\sigma_{\mathrm{xy}}^{\downarrow}$. Thus, the spin polarization-dependent conductivities $\sigma_{\mathrm{xy}}^{\uparrow}$ and $\sigma_{\mathrm{xy}}^{\downarrow}$ can be calculated by taking the integral of $\Omega_{\mathrm{z}}^{\uparrow}$ and $\Omega_{\mathrm{z}}^{\downarrow}$ over the entire Brillouin zone (BZ). Manna et al., by calculating the Hall conductivity contribution of the majority and minority spin channels from bulk $\mathrm{Co}_{2} \mathrm{MnGa}$, found $\sigma_{\mathrm{xy}} \sim 1600 \Omega^{-1} \mathrm{~cm}^{-1}$ and $\Omega_{\mathrm{z}}^{\downarrow}$ is almost equal to 0 at the Fermi level $\left(E_{\mathrm{F}}\right)$ [81]. The value of $\sigma_{\mathrm{xy}}$ in these thin films is half that of bulk $\mathrm{Co}_{2} \mathrm{MnGa}$ [81]. However, interestingly almost the same AHA of the bulk reports can be obtained since the value of $\sigma_{\mathrm{xx}}$ in this thin film is relatively smaller than in ref.[81].

To understand the influence of spin-dependent electrons for $\sigma_{\mathrm{xy}}$ of thin films with different thickness, my collaborators, Dr. Yuefeng Yin and A/Prof. Nikhil Medhekar from Monash University, calculate the averaged $z \mathrm{com}$ ponent of the Berry curvature (total Berry curvature/number of slabs) and surface band structure from majority spin electrons and minority spin electrons near the Fermi level in the $k_{\mathrm{x}}-k_{\mathrm{y}}$ plane, respectively, as shown in Figure 6.7. For 123 primitive cell $(50.1 \mathrm{~nm})$ thick slab of $\mathrm{Co}_{2} \mathrm{MnGa}, \Omega_{\mathrm{z}}^{\uparrow} \gg \Omega_{\mathrm{z}}^{\downarrow}$ (see Figures 6.7(b) and (d)), meaning $\sigma_{\mathrm{xy}}^{\uparrow} \gg \sigma_{\mathrm{xy}}^{\downarrow}$ and suggesting that the Berry curvature contribution to $\sigma_{\mathrm{xy}}$ from the majority spin electrons is much higher than that from minority spin electrons, which is the same as for bulk $\mathrm{Co}_{2} \mathrm{MnGa}$.

Recent work has confirmed this by spin-resolved angle-resolved photoelectron spectroscopy on a $50 \mathrm{~nm} \mathrm{Co}_{2} \mathrm{MnGa}$ thin film [177]. The magnitude of $\Omega_{\mathrm{z}}^{\uparrow}$ of this thick $\mathrm{Co}_{2} \mathrm{MnGa}$ film is close to bulk values and is consistent with $\sigma_{\mathrm{xy}}$ values calculated by others [81,77, 80], suggesting films of $\sim 50 \mathrm{~nm}$ thickness still show the bulk-like properties, like the large $\sigma_{\mathrm{xy}}$ originating from the non-trivial topology of the band structure with large 
intrinsic Berry curvature. The $\Omega_{\mathrm{z}}^{\uparrow}$ for the $50 \mathrm{~nm}$ film in Figure 6.7 is complicated because it includes multiple 'slabs' in the calculation, and at $50 \mathrm{~nm}$ there is not yet enough overlap of the states so that continuous bulk-like bands are formed. Once the thickness is decreased to $4.9 \mathrm{~nm}$ (12 primitive cells), $\Omega_{\mathrm{z}}^{\uparrow}$ drops significantly (see Figures 6.7(b) and (d). This agrees with the experimental observation that $\sigma_{\mathrm{xy}}$ reduces when film thickness decreases.

Figures 6.7(e) and (f) summarises the $\Omega_{\mathrm{z}}^{\uparrow}$ and $\Omega_{\mathrm{z}}^{\downarrow}$ as a function of $k$ along the $\Gamma$ - line for various thicknesses. The extent of Berry curvature reduction for majority spins is more significant than that for minority spins. Once the thickness is down to $20 \mathrm{~nm}$, the $\Omega_{\mathrm{z}}^{\uparrow}$ slightly drops, but it is still close to the bulk value indicating that $\sigma_{\mathrm{xy}}$ is robust down to this thickness. Below 15 $\mathrm{nm}$, the role of the surface becomes more important, which could result in extrinsic side-jump scattering and might suppress the effect of the Weyl nodes on the magnetotransport. What's more, there is a 3.27\% lattice mismatch between $\mathrm{Co}_{2} \mathrm{MnGa}$ and the $\mathrm{MgO}$ substrate. Though the stress in the $50 \mathrm{~nm}$ film is almost fully relaxed due to the post-annealing, there could be a tetragonal distortion at low thickness which might induce an energy shift of the Weyl nodes [178] or shift the electron density-of-states [179]. Note the very different maxima used in the scales of Figures 6.7(a) - (d) vs Figures 6.7(e) - (f), so that the main features can be seen in Figures 6.7(a) (d), and the very large spikes can be seen in Figures 6.7(e) - (f).

\subsection{Discussion and conclusions}

By comparing to previously reported results of thin films, including Heusler alloys and classic ferromagnetic transition metal and alloys, the AHA in $\mathrm{Co}_{2} \mathrm{MnGa}$ shows a large value, $11.4 \%$, and also a comparatively large Hall conductivity $775 \Omega^{-1} \mathrm{~cm}^{-1}$, as shown in Figure 6.8. The reported results were taken or calculated from Table 6.1. To obtain a large AHA, $\sigma_{\mathrm{xy}}$ needs to be large, or $\sigma_{\mathrm{xx}}$ needs to be small, but large $\sigma_{\mathrm{xy}}$ signal with very good 


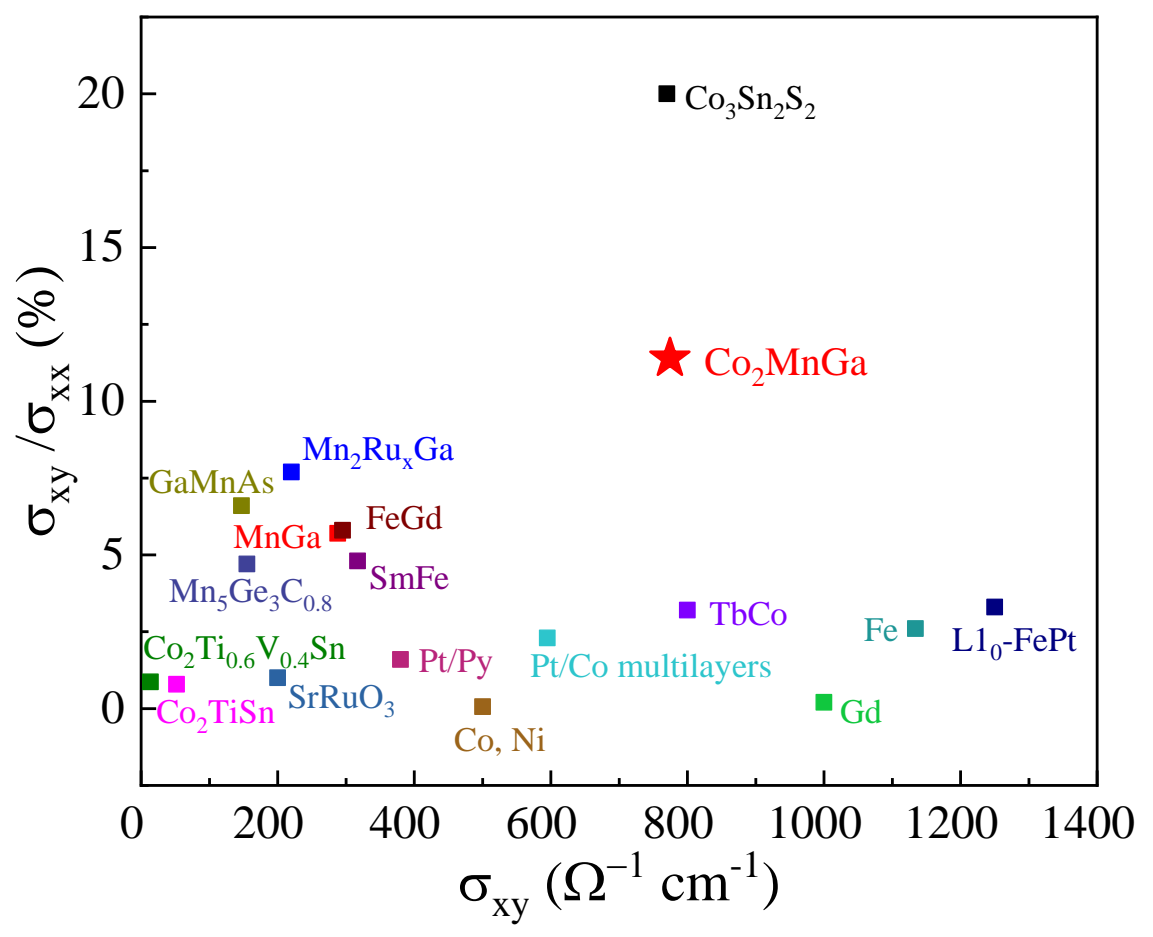

Figure 6.8: $\sigma_{\mathrm{xy}}$-dependent AHA of this result in comparison with previously reported results for other thin films.

signal-to-noise ratio is necessary for logic or memory hybrid CMOS/AHE devices [184] and AHE sensors [186]. Near the dirty regime[45] with a small $\sigma_{\mathrm{xx}}$, however, $\sigma_{\mathrm{xy}}$ is suppressed more drastically than $\sigma_{\mathrm{xx}}$, due to quasiparticle damping or spoiling of the resonance condition [187]. For ferromagnetic systems, both $\sigma_{\mathrm{xy}}$ and $\sigma_{\mathrm{xy}}$ are either large or small, and therefore the AHA is usually not very large. Though $\mathrm{Co}_{3} \mathrm{Sn}_{2} \mathrm{~S}_{2}$ thin films have a giant AHA 20\%, it shows a low Curie temperature $T_{\mathrm{c}}=177 \mathrm{~K}$ as mentioned before. Even for exceptions such as the compensated Heusler ferrimagnet $\mathrm{Mn}_{2-x} \mathrm{Ru}_{x} \mathrm{Ga}$, which has a large AHA 7.7\% at 300K, it displays a small $\sigma_{\mathrm{xy}}$, around $220 \Omega^{-1} \mathrm{~cm}^{-1}$ [52].

In summary, high quality epitaxial films of the topological semimetal $\mathrm{Co}_{2} \mathrm{MnGa}$ were prepared with thicknesses from 5 to $50 \mathrm{~nm}$ on $\mathrm{MgO}$ sub- 


\begin{tabular}{|c|c|c|c|c|}
\hline Materials & $\sigma_{\mathrm{xx}}$ & $\sigma_{\mathrm{xy}}$ & AHA & ref. \\
\hline $\mathrm{MnGa}$ & 5100 & 288 & 5.71 & [11] \\
\hline $\mathrm{Mn}_{2} \mathrm{Ru}_{x} \mathrm{Ga}$ & 2900 & 220 & 7.7 & [180] \\
\hline $\mathrm{Co}_{2} \mathrm{TiSn}$ & 6578.5 & 51.9 & 0.79 & [181] \\
\hline $\mathrm{Co}_{2} \mathrm{Ti}_{0.6} \mathrm{~V}_{0.4} \mathrm{Sn}$ & 1602.4 & 13.9 & 0.87 & [181] \\
\hline$L 1_{0}-\mathrm{FePt}$ & 37900 & 1250 & 3.3 & [11] \\
\hline TbCo & 25000 & 800 & 3.2 & [11] \\
\hline SmFe & 6600 & 317 & 4.8 & [11] \\
\hline FeGd & 5111 & 295 & 5.8 & [182] \\
\hline GaMnAs & 2200 & 147 & 6.6 & [11] \\
\hline $\mathrm{SrRuO}_{3}$ & 20000 & 200 & 1 & [11] \\
\hline $\mathrm{Fe}$ & 43600 & 1134 & 2.6 & [97] \\
\hline $\mathrm{Co}, \mathrm{Ni}$ & 100000 & 500 & 0.06 & [97] \\
\hline $\mathrm{Gd}$ & 500000 & 1000 & 0.2 & [97] \\
\hline $\mathrm{Pt} / \mathrm{Py}$ & 23800 & 380 & 1.6 & [183] \\
\hline $\mathrm{Pt} / \mathrm{Co}$ multilayers & 24376 & 595 & 2.3 & [184] \\
\hline $\mathrm{Mn}_{5} \mathrm{Ge}_{3} \mathrm{C}_{0.8}$ & 3326 & 155 & 4.7 & [185] \\
\hline $\mathrm{Co}_{3} \mathrm{Sn}_{2} \mathrm{~S}_{2}$ & 3850 & 770 & 20 & [74] \\
\hline $\mathrm{Co}_{2} \mathrm{MnGa}$ & 6805.2 & 774.4 & 11.4 & this work \\
\hline
\end{tabular}

Table 6.1: Summary of $\sigma_{\mathrm{xx}}, \sigma_{\mathrm{xy}}$, and AHA of magnetic thin films. Only the maximum values of AHA are adopted here.

strates and established they have a similar large AHA at room temperature as in bulk crystals. For the $50 \mathrm{~nm}$ thick film, it shows large $\sigma_{\mathrm{xy}}$ $\sim 775 \Omega^{-1} \mathrm{~cm}^{-1}$ and AHA $11.4 \%$ at $113 \mathrm{~K}$. The origin of the large $\sigma_{\mathrm{xy}}$ is the large net Berry curvature near the $E_{\mathrm{F}}$ due to the non-trivial topology of band structure of $\mathrm{Co}_{2} \mathrm{MnGa}$, and still shows a large AHA 9.7\% at 300 $\mathrm{K}$. The bulk-like properties are robust, for film thicknesses down to 20 $\mathrm{nm}$. Once the thickness is decreased below $20 \mathrm{~nm}, \sigma_{\mathrm{xy}}$ suddenly drops a lot with a slight change of $\sigma_{\mathrm{xx}}$, with a corresponding decrease in the 
AHA. From the band structure calculations, this decrease can be ascribe to the reduction of the majority spin electron Berry curvature. These results confirm that $\mathrm{Co}_{2} \mathrm{MnGa}$ is a topological magnetic material in which tuning of the Berry curvature is possible, as observed in the anomalous Hall behaviour. The opportunity to control the Berry curvature in materials provides a way to realize topo-spintronic logic or memory devices and AHE sensors that operate at room temperature. 


\section{Chapter 7}

\section{Summary and outlook}

\subsection{Summary}

This thesis presents comprehensive research on the magnetic properties, skyrmion generation and anomalous Hall effect for Heusler alloy thin films which show great potential for spintronics applications. Before discussing the experimental results, the required background and techniques are introduced.

Firstly, by carefully calibrate the thickness of each layer, the perpendicular magnetic anisotropy has been achieved in $\mathrm{MgO} / \mathrm{Mn}_{2} \mathrm{CoAl} / \mathrm{Pd}$ trilayers. The domain structures and effective magnetic anisotropy can be designed by fine tuning the thickness of Pd.

Secondly, based on the understanding of magnetic properties of the $\mathrm{MgO} / \mathrm{Mn}_{2} \mathrm{CoAl} / \mathrm{Pd}$ trilayers, the generation of skyrmions by applying an external in-plane field was investigated. With an IP field, skyrmions can be gradually generated from both collapse of the labyrinth domains and nucleation from uniformly ferromagnetic regions due to the reduction of $K_{\text {eff }}$. Initially, in a slightly weak PMA regime, skyrmions are formed from labyrinth domains. Further increasing the IP field reduces $K_{\text {eff }}$ which weakens the PMA and skyrmions start to form from uniformly ferromagnetic regions directly. Further, by doing fractal analysis, a magnetic phase 
diagram was established that clearly identifies the two different types of skyrmion formation, as a function of fractal dimension and IP field. The processes of evolution of magnetic domains and skyrmions can be described by the fractal dimension. Finally, an increasing IP field causing an effective increase in the critical material parameter $\kappa$ was found, which increases the skyrmion density up to a point beyond which the effect of a repulsive skyrmion-skyrmion interaction becomes dominant. These results show that skyrmions can be gradually generated with an assisted IP field and that fractal analysis is a potentially powerful tool to analyse the evolution of magnetic domains and skyrmions.

Thirdly, instead of using an in-plane field, the ionic liquid gating method was employed to create skyrmions for the trilayer in a non-skyrmion thickness regime. Non-volatile skyrmions can be generated at a negative gate voltage in films with a weak PMA. For the thin films with intermediate PMA, samples can be trained to the skyrmion regime by cycling voltages. The mechanism for both gating methods is that oxygen ions diffuse into the Pd layer and form a $\mathrm{PdO}_{x}$ layer, which induces a significant reduction of effective magnetic anisotropy, with an extremely high anisotropy field tunability. Also, volatile skyrmions can be created at a small positive gate voltage after applying a trigger gate voltage to etch away the native oxide $\mathrm{PdO}_{x}$ layer. Without $\mathrm{PdO}_{x}$ shielding, an electrostatic charge accumulation happens at the surface and induces an in-plane net Rashba magnetic field. These results strongly demonstrate that ILG is a powerful and versatile technique towards the generation of skyrmions and skyrmion-based applications.

Finally, the thickness dependence of anomalous Hall angle was systematically studied in Weyl semimetal $\mathrm{Co}_{2} \mathrm{MnGa}$ thin films which can be used as a spin-orbit torque generator to manipulate skyrmions. The high quality epitaxial films of $\mathrm{Co}_{2} \mathrm{MnGa}$ were prepared with thicknesses from 5 to $50 \mathrm{~nm}$ on $\mathrm{MgO}$ substrates. For the $50-\mathrm{nm}$ thick film, it shows a large $\sigma_{\mathrm{xy}} \sim 775 \Omega^{-1} \mathrm{~cm}^{-1}$ and AHA $11.4 \%$ at $113 \mathrm{~K}$. The origin of the large $\sigma_{\mathrm{xy}}$ is 
the large net Berry curvature near the $E_{\mathrm{F}}$ due to the non-trivial topology of band structure of $\mathrm{Co}_{2} \mathrm{MnGa}$, and still shows a large AHA 9.7\% at 300 $\mathrm{K}$. The bulk-like properties are robust, for film thicknesses down to $20 \mathrm{~nm}$. Once the thickness is decreased below $20 \mathrm{~nm}$, the $\sigma_{\mathrm{xy}}$ suddenly drops a lot with a slight change of $\sigma_{\mathrm{xx}}$, with a corresponding decrease in the AHA. From the band structure calculations, the decrease of AHA can be ascribed to the reduction of the majority spin electron Berry curvature. These results confirm that $\mathrm{Co}_{2} \mathrm{MnGa}$ is a topological magnetic material in which tuning of the Berry curvature is possible, as observed in the anomalous Hall behaviour. The opportunity to control the Berry curvature in materials provides a way to realize topo-spintronic logic or memory devices and AHE sensors that operate at room temperature.

\subsection{Outlook}

As a future task, it remains to experimentally confirm the magnetic chiral texture of skyrmions in $\mathrm{MgO} / \mathrm{Mn}_{2} \mathrm{CoAl} / \mathrm{Pd}$ system. In this thesis, the Néel type of domain wall has been verified by asymmetrical magnetic bubble expansion so that the skyrmions should be exhibit the same type of texture. The directly electric experimental proof used to confirm the magnetic chiral texture of skyrmions is skyrmion Hall effect in which the skyrmion experiences a transverse deflection analogous to charged particles in the classical Hall effect [188]. Also, it would be appealing to manipulate skyrmions by anomalous spin-orbit torque induced by $\mathrm{Co}_{2} \mathrm{MnGa}$, which is a new way to drive skyrmion motion. It is worth to compare the energy efficiency and critical current density of this method with traditional spin-orbit torque originating from heavy metal. 


\section{Bibliography}

[1] K. Wang, J. Alzate, and P. K. Amiri, "Low-power non-volatile spintronic memory: STT-RAM and beyond," Journal of Physics D: Applied Physics, vol. 46, no. 7, p. 074003, 2013.

[2] A. Hirohata, K. Yamada, Y. Nakatani, I.-L. Prejbeanu, B. Diény, P. Pirro, and B. Hillebrands, "Review on spintronics: Principles and device applications," Journal of Magnetism and Magnetic Materials, vol. 509, p. 166711, 2020.

[3] B. Dieny, I. L. Prejbeanu, K. Garello, P. Gambardella, P. Freitas, R. Lehndorff, W. Raberg, U. Ebels, S. O. Demokritov, J. Akerman, et al., "Opportunities and challenges for spintronics in the microelectronics industry," Nature Electronics, vol. 3, no. 8, pp. 446-459, 2020.

[4] J. Heidecker, "MRAM technology status," tech. rep., National Aeronautics and Space, 2013.

[5] B. Tudu and A. Tiwari, "Recent developments in perpendicular magnetic anisotropy thin films for data storage applications," Vacuum, vol. 146, pp. 329-341, 2017.

[6] A. Fert, V. Cros, and J. Sampaio, "Skyrmions on the track," Nature Nanotechnology, vol. 8, no. 3, pp. 152-156, 2013. 
[7] W. Jiang, G. Chen, K. Liu, J. Zang, S. G. Te Velthuis, and A. Hoffmann, "Skyrmions in magnetic multilayers," Physics Reports, vol. 704, pp. 1-49, 2017.

[8] W. Jiang, P. Upadhyaya, W. Zhang, G. Yu, M. B. Jungfleisch, F. Y. Fradin, J. E. Pearson, Y. Tserkovnyak, K. L. Wang, O. Heinonen, et al., "Blowing magnetic skyrmion bubbles," Science, vol. 349, no. 6245, pp. 283-286, 2015.

[9] C. Moreau-Luchaire, C. Moutafis, N. Reyren, J. Sampaio, C. Vaz, N. Van Horne, K. Bouzehouane, K. Garcia, C. Deranlot, P. Warnicke, et al., "Additive interfacial chiral interaction in multilayers for stabilization of small individual skyrmions at room temperature," Nature Nanotechnology, vol. 11, no. 5, pp. 444-448, 2016.

[10] K. Manna, Y. Sun, L. Muechler, J. Kübler, and C. Felser, "Heusler, Weyl and Berry," Nature Reviews Materials, vol. 3, no. 8, pp. 244-256, 2018.

[11] E. Liu, Y. Sun, N. Kumar, L. Muechler, A. Sun, L. Jiao, S.-Y. Yang, D. Liu, A. Liang, Q. Xu, et al., "Giant anomalous Hall effect in a ferromagnetic kagome-lattice semimetal," Nature Physics, vol. 14, no. 11, pp. 1125-1131, 2018.

[12] Y. Zhang, Y. Yin, G. Dubuis, T. Butler, N. V. Medhekar, and S. Granville, "Berry curvature origin of the thickness-dependent anomalous Hall effect in a ferromagnetic Weyl semimetal," npj Quantum Materials, vol. 6, no. 1, pp. 1-8, 2021.

[13] M. N. Baibich, J. M. Broto, A. Fert, F. N. Van Dau, F. Petroff, P. Etienne, G. Creuzet, A. Friederich, and J. Chazelas, "Giant magnetoresistance of (001) Fe/(001) Cr magnetic superlattices," Physical Review Letters, vol. 61, no. 21, p. 2472, 1988. 
[14] G. Binasch, P. Grünberg, F. Saurenbach, and W. Zinn, "Enhanced magnetoresistance in layered magnetic structures with antiferromagnetic interlayer exchange," Physical Review B, vol. 39, no. 7, p. $4828,1989$.

[15] M. Julliere, "Tunneling between ferromagnetic films," Physics Letters A, vol. 54, no. 3, pp. 225-226, 1975.

[16] S. Yuasa, T. Nagahama, A. Fukushima, Y. Suzuki, and K. Ando, "Giant room-temperature magnetoresistance in single-crystal $\mathrm{Fe} / \mathrm{MgO} / \mathrm{Fe}$ magnetic tunnel junctions," Nature Materials, vol. 3, no. 12, pp. 868-871, 2004.

[17] S. S. Parkin, C. Kaiser, A. Panchula, P. M. Rice, B. Hughes, M. Samant, and S.-H. Yang, "Giant tunnelling magnetoresistance at room temperature with $\mathrm{MgO}(100)$ tunnel barriers," Nature Materials, vol. 3, no. 12, pp. 862-867, 2004.

[18] G. Ju, Y. Peng, E. K. Chang, Y. Ding, A. Q. Wu, X. Zhu, Y. Kubota, T. J. Klemmer, H. Amini, L. Gao, et al., "High density heat-assisted magnetic recording media and advanced characterization-progress and challenges," IEEE Transactions on Magnetics, vol. 51, no. 11, pp. 1-9, 2015.

[19] L. Berger, "Emission of spin waves by a magnetic multilayer traversed by a current," Physical Review B, vol. 54, no. 13, p. 9353, 1996.

[20] J. C. Slonczewski, "Current-driven excitation of magnetic multilayers," Journal of Magnetism and Magnetic Materials, vol. 159, no. 1-2, pp. L1-L7, 1996.

[21] A. Khvalkovskiy, D. Apalkov, S. Watts, R. Chepulskii, R. Beach, A. Ong, X. Tang, A. Driskill-Smith, W. Butler, P. Visscher, et al., "Basic principles of STT-MRAM cell operation in memory arrays," Journal of Physics D: Applied Physics, vol. 46, no. 7, p. 074001, 2013. 
[22] A. Manchon, J. Železnỳ, I. M. Miron, T. Jungwirth, J. Sinova, A. Thiaville, K. Garello, and P. Gambardella, "Current-induced spin-orbit torques in ferromagnetic and antiferromagnetic systems," Reviews of Modern Physics, vol. 91, no. 3, p. 035004, 2019.

[23] L. Liu, C.-F. Pai, Y. Li, H. Tseng, D. Ralph, and R. Buhrman, "Spintorque switching with the giant spin Hall effect of tantalum," Science, vol. 336, no. 6081, pp. 555-558, 2012.

[24] S. S. Parkin, M. Hayashi, and L. Thomas, "Magnetic domain-wall racetrack memory," Science, vol. 320, no. 5873, pp. 190-194, 2008.

[25] M. Hayashi, L. Thomas, C. Rettner, R. Moriya, Y. B. Bazaliy, and S. S. Parkin, "Current driven domain wall velocities exceeding the spin angular momentum transfer rate in permalloy nanowires," Physical Review Letters, vol. 98, no. 3, p. 037204, 2007.

[26] I. Dzyaloshinsky, "A thermodynamic theory of "weak" ferromagnetism of antiferromagnetics," Journal of Physics and Chemistry of Solids, vol. 4, no. 4, pp. 241-255, 1958.

[27] T. Moriya, "Anisotropic superexchange interaction and weak ferromagnetism," Physical Review, vol. 120, no. 1, p. 91, 1960.

[28] J. Sinova, S. O. Valenzuela, J. Wunderlich, C. Back, and T. Jungwirth, "Spin Hall effects," Reviews of Modern Physics, vol. 87, no. 4, p. 1213, 2015.

[29] S. S. Parkin and S.-H. Yang, "Memory on the racetrack," Nature Nanotechnology, vol. 10, no. 3, pp. 195-198, 2015.

[30] S. S. Parkin, "Data in the fast lanes of racetrack memory," Scientific American, vol. 300, no. 6, pp. 76-81, 2009. 
[31] X. Yu, Y. Onose, N. Kanazawa, J. Park, J. Han, Y. Matsui, N. Nagaosa, and Y. Tokura, "Real-space observation of a two-dimensional skyrmion crystal," Nature, vol. 465, no. 7300, pp. 901-904, 2010.

[32] S. Seki, X. Yu, S. Ishiwata, and Y. Tokura, "Observation of skyrmions in a multiferroic material," Science, vol. 336, no. 6078, pp. 198-201, 2012.

[33] K. Karube, J. White, N. Reynolds, J. Gavilano, H. Oike, A. Kikkawa, F. Kagawa, Y. Tokunaga, H. M. Rønnow, Y. Tokura, et al., "Robust metastable skyrmions and their triangular-square lattice structural transition in a high-temperature chiral magnet," Nature Materials, vol. 15, no. 12, pp. 1237-1242, 2016.

[34] N. Romming, C. Hanneken, M. Menzel, J. E. Bickel, B. Wolter, K. von Bergmann, A. Kubetzka, and R. Wiesendanger, "Writing and deleting single magnetic skyrmions," Science, vol. 341, no. 6146, pp. 636639, 2013.

[35] X. Zhang, Y. Zhou, K. M. Song, T.-E. Park, J. Xia, M. Ezawa, X. Liu, W. Zhao, G. Zhao, and S. Woo, "Skyrmion-electronics: writing, deleting, reading and processing magnetic skyrmions toward spintronic applications," Journal of Physics: Condensed Matter, vol. 32, no. 14, p. 143001, 2020.

[36] P. Carcia, A. Meinhaldt, and A. Suna, "Perpendicular magnetic anisotropy in Pd/Co thin film layered structures," Applied Physics Letters, vol. 47, no. 2, pp. 178-180, 1985.

[37] P. Carcia, "Perpendicular magnetic anisotropy in $\mathrm{Pd} / \mathrm{Co}$ and $\mathrm{Pt} / \mathrm{Co}$ thin-film layered structures," Journal of Applied Physics, vol. 63, no. 10, pp. 5066-5073, 1988. 
[38] F. Den Broeder, W. Hoving, and P. Bloemen, "Magnetic anisotropy of multilayers," Journal of Magnetism and Magnetic Materials, vol. 93, pp. 562-570, 1991.

[39] J. Stöhr, "Exploring the microscopic origin of magnetic anisotropies with X-ray magnetic circular dichroism (XMCD) spectroscopy," Journal of Magnetism and Magnetic Materials, vol. 200, no. 1-3, pp. 470$497,1999$.

[40] S. Monso, B. Rodmacq, S. Auffret, G. Casali, F. Fettar, B. Gilles, B. Dieny, and P. Boyer, "Crossover from in-plane to perpendicular anisotropy in $\mathrm{Pt} / \mathrm{CoFe} / \mathrm{AlO}_{x}$ sandwiches as a function of $\mathrm{Al}$ oxidation: A very accurate control of the oxidation of tunnel barriers," Applied Physics Letters, vol. 80, no. 22, pp. 4157-4159, 2002.

[41] A. Manchon, C. Ducruet, L. Lombard, S. Auffret, B. Rodmacq, B. Dieny, S. Pizzini, J. Vogel, V. Uhlír, M. Hochstrasser, et al., "Analysis of oxygen induced anisotropy crossover in $\mathrm{Pt} / \mathrm{Co} / \mathrm{MO}_{x}$ trilayers," Journal of Applied Physics, vol. 104, no. 4, p. 043914, 2008.

[42] H. Yang, M. Chshiev, B. Dieny, J. Lee, A. Manchon, and K. Shin, "First-principles investigation of the very large perpendicular magnetic anisotropy at $\mathrm{Fe} / \mathrm{MgO}$ and $\mathrm{Co} / \mathrm{MgO}$ interfaces," Physical Review B, vol. 84, no. 5, p. 054401, 2011.

[43] J. M. Coey, Magnetism and magnetic materials. Cambridge university press, 2010.

[44] I. M. Miron, T. Moore, H. Szambolics, L. D. Buda-Prejbeanu, S. Auffret, B. Rodmacq, S. Pizzini, J. Vogel, M. Bonfim, A. Schuhl, et al., "Fast current-induced domain-wall motion controlled by the Rashba effect," Nature Materials, vol. 10, no. 6, pp. 419-423, 2011. 
[45] K.-S. Ryu, L. Thomas, S.-H. Yang, and S. S. Parkin, "Chiral spin torque at magnetic domain walls," Nature Nanotechnology, vol. 8, no. 7, pp. 527-533, 2013.

[46] A. Fert, N. Reyren, and V. Cros, "Magnetic skyrmions: advances in physics and potential applications," Nature Reviews Materials, vol. 2, no. 7, p. 17031, 2017.

[47] K. Everschor-Sitte, J. Masell, R. M. Reeve, and M. Kläui, “Perspective: Magnetic skyrmions-overview of recent progress in an active research field," Journal of Applied Physics, vol. 124, no. 24, p. 240901, 2018.

[48] F. Jonietz, S. Mühlbauer, C. Pfleiderer, A. Neubauer, W. Münzer, A. Bauer, T. Adams, R. Georgii, P. Böni, R. A. Duine, et al., "Spin transfer torques in $\mathrm{MnSi}$ at ultralow current densities," Science, vol. 330, no. 6011, pp. 1648-1651, 2010.

[49] X. Yu, N. Kanazawa, W. Zhang, T. Nagai, T. Hara, K. Kimoto, Y. Matsui, Y. Onose, and Y. Tokura, "Skyrmion flow near room temperature in an ultralow current density," Nature Communications, vol. 3, no. 1, pp. 1-6, 2012.

[50] S. Woo, K. Litzius, B. Krüger, M.-Y. Im, L. Caretta, K. Richter, M. Mann, A. Krone, R. M. Reeve, M. Weigand, et al., "Observation of room-temperature magnetic skyrmions and their current-driven dynamics in ultrathin metallic ferromagnets," Nature Materials, vol. 15, no. 5, pp. 501-506, 2016.

[51] F. Heusler, "Ueber magnetische manganlegierungen," Verh. Dtsch. Phys. Ges., vol. 5, p. 219, 1903.

[52] O. Heusler, "Kristallstruktur und ferromagnetismus der manganaluminium-kupferlegierungen," Annalen der Physik, vol. 411, no. 2, pp. 155-201, 1934. 
[53] A. J. Bradley and J. Rodgers, "The crystal structure of the heusler alloys," Proceedings of the royal society of london. Series A, Containing Papers of a Mathematical and Physical Character, vol. 144, no. 852, pp. 340-359, 1934.

[54] T. Graf, C. Felser, and S. S. Parkin, "Simple rules for the understanding of heusler compounds," Progress in solid state chemistry, vol. 39, no. 1, pp. 1-50, 2011.

[55] A. Hirohata, W. Frost, M. Samiepour, and J.-y. Kim, "Perpendicular magnetic anisotropy in heusler alloy films and their magnetoresistive junctions," Materials, vol. 11, no. 1, p. 105, 2018.

[56] A. Hirohata, T. Huminiuc, J. Sinclair, H. Wu, M. Samiepour, G. Vallejo-Fernandez, K. O'Grady, J. Balluf, M. Meinert, G. Reiss, et al., "Development of antiferromagnetic heusler alloys for the replacement of iridium as a critically raw material," Journal of Physics D: Applied Physics, vol. 50, no. 44, p. 443001, 2017.

[57] Y. Sakuraba, J. Nakata, M. Oogane, H. Kubota, Y. Ando, A. Sakuma, and T. Miyazaki, "Huge spin-polarization of $\mathrm{L}_{1}$-ordered $\mathrm{Co}_{2} \mathrm{MnSi}$ epitaxial Heusler alloy film," Japanese Journal of Applied Physics, vol. 44, no. 8L, p. L1100, 2005.

[58] W. E. Pickett and J. S. Moodera, "Half metallic magnets," Physics Today, vol. 54, no. 5, pp. 39-45, 2001.

[59] G. Strijkers, Y. Ji, F. Yang, C. Chien, and J. Byers, "Andreev reflections at metal/superconductor point contacts: Measurement and analysis," Physical Review B, vol. 63, no. 10, p. 104510, 2001.

[60] H.-x. Liu, Y. Honda, T. Taira, K.-i. Matsuda, M. Arita, T. Uemura, and M. Yamamoto, "Giant tunneling magnetoresistance in epitaxial $\mathrm{Co}_{2} \mathrm{MnSi} / \mathrm{MgO} / \mathrm{Co}_{2} \mathrm{MnSi}$ magnetic tunnel junctions by half- 
metallicity of $\mathrm{Co}_{2} \mathrm{MnSi}$ and coherent tunneling," Applied Physics Letters, vol. 101, no. 13, p. 132418, 2012.

[61] I. Galanakis and P. H. Dederichs, "Half-metallicity and SlaterPauling behavior in the ferromagnetic Heusler alloys," in Halfmetallic Alloys, pp. 1-39, Springer, 2005.

[62] I. Galanakis, P. Dederichs, and N. Papanikolaou, "Slater-Pauling behavior and origin of the half-metallicity of the full-Heusler alloys," Physical Review B, vol. 66, no. 17, p. 174429, 2002.

[63] R. De Groot, F. Mueller, P. Van Engen, and K. Buschow, "New class of materials: half-metallic ferromagnets," Physical Review Letters, vol. 50, no. 25, p. 2024, 1983.

[64] J. Kübler, A. William, and C. Sommers, "Formation and coupling of magnetic moments in Heusler alloys," Physical Review B, vol. 28, no. 4, p. 1745, 1983.

[65] S. Fujii, S. Sugimura, S. Asano, et al., "Hyperfine fields and electronic structures of the Heusler alloys $\mathrm{Co}_{2} \mathrm{MnX}(\mathrm{X}=\mathrm{Al}, \mathrm{Ga}, \mathrm{Si}, \mathrm{Ge}, \mathrm{Sn})$," Journal of Physics: Condensed Matter, vol. 2, no. 43, p. 8583, 1990.

[66] S. Ouardi, G. H. Fecher, C. Felser, and J. Kübler, "Realization of spin gapless semiconductors: The Heusler compound $\mathrm{Mn}_{2} \mathrm{CoAl}$," Physical Review Letters, vol. 110, no. 10, p. 100401, 2013.

[67] M. E. Jamer, B. A. Assaf, T. Devakul, and D. Heiman, "Magnetic and transport properties of $\mathrm{Mn}_{2} \mathrm{CoAl}$ oriented films," Applied Physics Letters, vol. 103, no. 14, p. 142403, 2013.

[68] R. G. Buckley, T. Butler, C. Pot, N. M. Strickland, and S. Granville, "Exploring disorder in the spin gapless semiconductor $\mathrm{Mn}_{2} \mathrm{CoAl}$," Materials Research Express, vol. 6, no. 10, p. 106113, 2019. 
[69] N. Sun, Y. Zhang, H. Fu, W. Che, C. You, and R. Shan, "Perpendicular magnetic anisotropy in $\mathrm{Mn}_{2} \mathrm{CoAl}$ thin film," AIP Advances, vol. 6, no. 1, p. 015006, 2016.

[70] B. Ludbrook, G. Dubuis, A.-H. Puichaud, B. Ruck, and S. Granville, "Nucleation and annihilation of skyrmions in $\mathrm{Mn}_{2} \mathrm{CoAl}$ observed through the topological Hall effect," Scientific Reports, vol. 7, no. 1, p. 13620, 2017.

[71] N. Armitage, E. Mele, and A. Vishwanath, "Weyl and Dirac semimetals in three-dimensional solids," Reviews of Modern Physics, vol. 90, no. 1, p. 015001, 2018.

[72] J. Hu, S.-Y. $\mathrm{Xu}, \mathrm{N} . \mathrm{Ni}$, and Z. Mao, "Transport of topological semimetals," Annual Review of Materials Research, vol. 49, pp. 207$252,2019$.

[73] P. Bruno, "Berry phase effects in magnetism," arXiv preprint condmat/0506270, 2005.

[74] K. Fujiwara, J. Ikeda, J. Shiogai, T. Seki, K. Takanashi, and A. Tsukazaki, "Ferromagnetic $\mathrm{Co}_{3} \mathrm{Sn}_{2} \mathrm{~S}_{2}$ thin films fabricated by co-sputtering," Japanese Journal of Applied Physics, vol. 58, no. 5, p. $050912,2019$.

[75] Z. Wang, M. Vergniory, S. Kushwaha, M. Hirschberger, E. Chulkov, A. Ernst, N. P. Ong, R. J. Cava, and B. A. Bernevig, "Time-reversalbreaking Weyl fermions in magnetic Heusler alloys," Physical Review Letters, vol. 117, no. 23, p. 236401, 2016.

[76] J. Kübler and C. Felser, "Weyl points in the ferromagnetic Heusler compound $\mathrm{Co}_{2} \mathrm{MnAl}$," Europhysics Letters, vol. 114, no. 4, p. 47005, 2016. 
[77] I. Belopolski, K. Manna, D. S. Sanchez, G. Chang, B. Ernst, J. Yin, S. S. Zhang, T. Cochran, N. Shumiya, H. Zheng, et al., "Discovery of topological Weyl fermion lines and drumhead surface states in a room temperature magnet," Science, vol. 365, no. 6459, pp. 1278 $1281,2019$.

[78] S. N. Guin, K. Manna, J. Noky, S. J. Watzman, C. Fu, N. Kumar, W. Schnelle, C. Shekhar, Y. Sun, J. Gooth, et al., "Anomalous Nernst effect beyond the magnetization scaling relation in the ferromagnetic Heusler compound $\mathrm{Co}_{2} \mathrm{MnGa}$," NPG Asia Materials, vol. 11, no. 1, pp. 1-9, 2019.

[79] A. Sakai, Y. P. Mizuta, A. A. Nugroho, R. Sihombing, T. Koretsune, M.-T. Suzuki, N. Takemori, R. Ishii, D. Nishio-Hamane, R. Arita, et al., "Giant anomalous Nernst effect and quantum-critical scaling in a ferromagnetic semimetal," Nature Physics, vol. 14, no. 11, pp. 1119-1124, 2018.

[80] J.-C. Tung and G.-Y. Guo, "High spin polarization of the anomalous Hall current in Co-based Heusler compounds," New Journal of Physics, vol. 15, no. 3, p. 033014, 2013.

[81] K. Manna, L. Muechler, T.-H. Kao, R. Stinshoff, Y. Zhang, J. Gooth, N. Kumar, G. Kreiner, K. Koepernik, R. Car, et al., "From colossal to zero: controlling the anomalous Hall effect in magnetic Heusler compounds via Berry curvature design," Physical Review X, vol. 8, no. 4, p. 041045, 2018.

[82] Y. Lu, J. Cai, H. Pan, and L. Sun, "Ultrasensitive anomalous Hall effect in $\mathrm{SiO}_{2} / \mathrm{Fe}-\mathrm{Pt} / \mathrm{SiO}_{2}$ sandwich structure films," Applied Physics Letters, vol. 100, no. 2, p. 022404, 2012.

[83] S. Iihama, T. Taniguchi, K. Yakushiji, A. Fukushima, Y. Shiota, S. Tsunegi, R. Hiramatsu, S. Yuasa, Y. Suzuki, and H. Kubota, "Spin- 
transfer torque induced by the spin anomalous Hall effect," Nature Electronics, vol. 1, no. 2, pp. 120-123, 2018.

[84] A. K. Nayak, V. Kumar, T. Ma, P. Werner, E. Pippel, R. Sahoo, F. Damay, U. K. Rößler, C. Felser, and S. S. Parkin, “Magnetic antiskyrmions above room temperature in tetragonal Heusler materials," Nature, vol. 548, no. 7669, pp. 561-566, 2017.

[85] L. Peng, R. Takagi, W. Koshibae, K. Shibata, K. Nakajima, T.h. Arima, N. Nagaosa, S. Seki, X. Yu, and Y. Tokura, "Controlled transformation of skyrmions and antiskyrmions in a noncentrosymmetric magnet," Nature Nanotechnology, vol. 15, no. 3, pp. 181-186, 2020.

[86] S. Husain, N. Sisodia, A. K. Chaurasiya, A. Kumar, J. P. Singh, B. S. Yadav, S. Akansel, K. H. Chae, A. Barman, P. Muduli, et al., "Observation of skyrmions at room temperature in $\mathrm{Co}_{2} \mathrm{FeAl}$ Heusler alloy ultrathin film heterostructures," Scientific Reports, vol. 9, no. 1, pp. 1$12,2019$.

[87] W. Akhtar, A. Hrabec, S. Chouaieb, A. Haykal, I. Gross, M. Belmeguenai, M. Gabor, B. Shields, P. Maletinsky, A. Thiaville, et al., "Current-induced nucleation and dynamics of skyrmions in a Co-based Heusler alloy," Physical Review Applied, vol. 11, no. 3, p. 034066, 2019.

[88] Y. Zhang, G. Dubuis, T. Butler, and S. Granville, “Fractal analysis of skyrmions generated by field-assisted fine-tuning of magnetic anisotropy," Physical Review Applied, vol. 15, no. 1, p. 014020, 2021.

[89] S. Z. Bisri, S. Shimizu, M. Nakano, and Y. Iwasa, "Endeavor of iontronics: from fundamentals to applications of ion-controlled electronics," Advanced Materials, vol. 29, no. 25, p. 1607054, 2017. 
[90] C. Navarro-Senent, A. Quintana, E. Menéndez, E. Pellicer, and J. Sort, "Electrolyte-gated magnetoelectric actuation: Phenomenology, materials, mechanisms, and prospective applications," APL Materials, vol. 7, no. 3, p. 030701, 2019.

[91] M. Weisheit, S. Fähler, A. Marty, Y. Souche, C. Poinsignon, and D. Givord, "Electric field-induced modification of magnetism in thin-film ferromagnets," Science, vol. 315, no. 5810, pp. 349-351, 2007.

[92] S. Zhao, L. Wang, Z. Zhou, C. Li, G. Dong, L. Zhang, B. Peng, T. Min, $\mathrm{Z}$. Hu, J. Ma, et al., "Ionic liquid gating control of spin reorientation transition and switching of perpendicular magnetic anisotropy," $A d-$ vanced Materials, vol. 30, no. 30, p. 1801639, 2018.

[93] L. H. Diez, Y. Liu, D. A. Gilbert, M. Belmeguenai, J. Vogel, S. Pizzini, E. Martinez, A. Lamperti, J. Mohammedi, A. Laborieux, et al., "Nonvolatile ionic modification of the Dzyaloshinskii-Moriya interaction," Physical Review Applied, vol. 12, no. 3, p. 034005, 2019.

[94] Y. Gan, "Atomic and subnanometer resolution in ambient conditions by atomic force microscopy," Surface Science Reports, vol. 64, no. 3, pp. 99-121, 2009.

[95] PPMS Horizontal Rotator. Quantum Design.

[96] N. Nagaosa, J. Sinova, S. Onoda, A. H. MacDonald, and N. P. Ong, "Anomalous Hall effect," Reviews of Modern Physics, vol. 82, no. 2, p. 1539, 2010.

[97] T. Miyasato, N. Abe, T. Fujii, A. Asamitsu, S. Onoda, Y. Onose, N. Nagaosa, and Y. Tokura, "Crossover behavior of the anomalous Hall effect and anomalous Nernst effect in itinerant ferromagnets," Physical Review Letters, vol. 99, no. 8, p. 086602, 2007. 
[98] C. R. Akouala, R. Kumar, S. Punugupati, C. L. Reynolds, J. G. Reynolds, E. J. Mily, J.-P. Maria, J. Narayan, and F. Hunte, "Planar Hall effect and anisotropic magnetoresistance in semiconducting and conducting oxide thin films," Applied Physics A, vol. 125, no. 5, p. 293, 2019.

[99] M. McElfresh, "Fundamentals of magnetism and magnetic measurements," Quantum Design, 1994.

[100] MPMS-XL manual. Quantum Design.

[101] Z. Yang and M. Scheinfein, "Combined three-axis surface magnetooptical Kerr effects in the study of surface and ultrathin-film magnetism," Journal of Applied Physics, vol. 74, no. 11, pp. 6810-6823, 1993.

[102] C. Tannous and J. Gieraltowski, "The Stoner-Wohlfarth model of ferromagnetism," European Journal of Physics, vol. 29, no. 3, p. 475, 2008.

[103] T. Wu, H. Fu, R. Hajjar, T. Suzuki, and M. Mansuripur, "Measurement of magnetic anisotropy constant for magneto-optical recording media: A comparison of several techniques," Journal of Applied Physics, vol. 73, no. 3, pp. 1368-1376, 1993.

[104] P. Shepley, A. Rushforth, M. Wang, G. Burnell, and T. Moore, "Modification of perpendicular magnetic anisotropy and domain wall velocity in $\mathrm{Pt} / \mathrm{Co} / \mathrm{Pt}$ by voltage-induced strain," Scientific Reports, vol. 5, no. 1, pp. 1-5, 2015.

[105] R. Lavrijsen, D. Hartmann, A. van den Brink, Y. Yin, B. Barcones, R. Duine, M. Verheijen, H. Swagten, and B. Koopmans, "Asymmetric magnetic bubble expansion under in-plane field in $\mathrm{Pt} / \mathrm{Co} / \mathrm{Pt}$ : Effect of interface engineering," Physical Review B, vol. 91, no. 10, p. 104414, 2015. 
[106] S.-G. Je, D.-H. Kim, S.-C. Yoo, B.-C. Min, K.-J. Lee, and S.-B. Choe, "Asymmetric magnetic domain-wall motion by the DzyaloshinskiiMoriya interaction," Physical Review B, vol. 88, no. 21, p. 214401, 2013.

[107] A. Hrabec, N. A. Porter, A. Wells, M. J. Benitez, G. Burnell, S. McVitie, D. McGrouther, T. A. Moore, and C. H. Marrows, "Measuring and tailoring the Dzyaloshinskii-Moriya interaction in perpendicularly magnetized thin films," Physical Review B, vol. 90, p. 020402, Jul 2014.

[108] "ImageJ - An open platform for scientific image analysis, https://imagej.net/welcome."

[109] K. Foroutan-pour, P. Dutilleul, and D. L. Smith, "Advances in the implementation of the box-counting method of fractal dimension estimation," Applied Mathematics and Computation, vol. 105, no. 2-3, pp. 195-210, 1999.

[110] D. Nečas and P. Klapetek, "Gwyddion: an open-source software for SPM data analysis," Open Physics, vol. 10, no. 1, pp. 181-188, 2012.

[111] S. Blundell, Magnetism In Condensed Matter. Oxford University Press, 2001.

[112] G. Xu, Y. Du, X. Zhang, H. Zhang, E. Liu, W. Wang, and G. Wu, "Magneto-transport properties of oriented $\mathrm{Mn}_{2} \mathrm{CoAl}$ films sputtered on thermally oxidized Si substrates," Applied Physics Letters, vol. 104, no. 24, p. 242408, 2014.

[113] K. Ueda, S. Hirose, and H. Asano, "Ambipolar transport in $\mathrm{Mn}_{2} \mathrm{CoAl}$ films by ionic liquid gating," Applied Physics Letters, vol. 110, no. 20, p. 202405, 2017. 
[114] Y.-C. Lau, Z. Chi, T. Taniguchi, M. Kawaguchi, G. Shibata, N. Kawamura, M. Suzuki, S. Fukami, A. Fujimori, H. Ohno, et al., "Giant perpendicular magnetic anisotropy in $\mathrm{Ir} / \mathrm{Co} / \mathrm{Pt}$ multilayers," Physical Review Materials, vol. 3, no. 10, p. 104419, 2019.

[115] G. Yu, P. Upadhyaya, X. Li, W. Li, S. K. Kim, Y. Fan, K. L. Wong, Y. Tserkovnyak, P. K. Amiri, and K. L. Wang, "Room-temperature creation and spin-orbit torque manipulation of skyrmions in thin films with engineered asymmetry," Nano Letters, vol. 16, no. 3, pp. 1981-1988, 2016.

[116] A. Soumyanarayanan, M. Raju, A. G. Oyarce, A. K. Tan, M.-Y. Im, A. P. Petrović, P. Ho, K. Khoo, M. Tran, C. Gan, et al., “Tunable roomtemperature magnetic skyrmions in $\mathrm{Ir} / \mathrm{Fe} / \mathrm{Co} / \mathrm{Pt}$ multilayers," $\mathrm{Na}$ ture Materials, vol. 16, no. 9, pp. 898-904, 2017.

[117] R. Tolley, S. Montoya, and E. Fullerton, "Room-temperature observation and current control of skyrmions in $\mathrm{Pt} / \mathrm{Co} / \mathrm{Os} / \mathrm{Pt}$ thin films," Physical Review Materials, vol. 2, no. 4, p. 044404, 2018.

[118] I. Lemesh, K. Litzius, M. Böttcher, P. Bassirian, N. Kerber, D. Heinze, J. Zázvorka, F. Büttner, L. Caretta, M. Mann, et al., “Current-induced skyrmion generation through morphological thermal transitions in chiral ferromagnetic heterostructures," Advanced Materials, vol. 30, no. 49, p. 1805461, 2018.

[119] B.-S. Han, D. Li, D.-J. Zheng, and Y. Zhou, "Fractal study of magnetic domain patterns," Physical Review B, vol. 66, no. 1, p. 014433, 2002.

[120] B. B. Mandelbrot, The fractal geometry of nature. Freeman, San Francisco, 1982.

[121] J. Attané, M. Tissier, A. Marty, and L. Vila, "Invasion percolation universality class and fractal geometry of magnetic domains," Physical Review B, vol. 82, no. 2, p. 024408, 2010. 
[122] S. Zhang, J. Zhang, Y. Wen, E. M. Chudnovsky, and X. Zhang, “Determination of chirality and density control of Néel-type skyrmions with in-plane magnetic field," Communications Physics, vol. 1, no. 1, p. 36, 2018.

[123] J. Hamrle, O. Gaier, S.-G. Min, B. Hillebrands, Y. Sakuraba, and Y. Ando, "Determination of exchange constants of heusler compounds by Brillouin light scattering spectroscopy: application to $\mathrm{Co}_{2} \mathrm{MnSi}$," Journal of Physics D: Applied Physics, vol. 42, no. 8, p. 084005, 2009.

[124] A. Jakobsson, P. Mavropoulos, E. Şaşığlu, S. Blügel, M. Ležaić, B. Sanyal, and I. Galanakis, "First-principles calculations of exchange interactions, spin waves, and temperature dependence of magnetization in inverse-Heusler-based spin gapless semiconductors," Physical Review B, vol. 91, no. 17, p. 174439, 2015.

[125] M. Heide, G. Bihlmayer, and S. Blügel, “Dzyaloshinskii-Moriya interaction accounting for the orientation of magnetic domains in ultrathin films: Fe/W (110)," Physical Review B, vol. 78, no. 14, p. 140403, 2008.

[126] B. Kaplan and G. Gehring, "The domain structure in ultrathin magnetic films," Journal of Magnetism and Magnetic Materials, vol. 128, no. 1-2, pp. 111-116, 1993.

[127] T. H. Johansen, A. V. Pan, and Y. M. Galperin, “Exact asymptotic behavior of magnetic stripe domain arrays," Physical Review B, vol. 87, no. 6, p. 060402, 2013.

[128] J. Takács, "A phenomenological mathematical model of hysteresis," COMPEL, 2001. 
[129] B. Mandelbrot, "How long is the coast of britain? statistical selfsimilarity and fractional dimension," Science, vol. 156, no. 3775, pp. 636-638, 1967.

[130] "https://imagej.nih.gov/ij/plugins/fraclac/flhelp/tutorials.htm."

[131] G. Peano, "Sur une courbe, qui remplit toute une aire plane," Mathematische Annalen, vol. 36, no. 1, pp. 157-160, 1890.

[132] H. Du, X. Zhao, F. N. Rybakov, A. B. Borisov, S. Wang, J. Tang, C. Jin, C. Wang, W. Wei, N. S. Kiselev, et al., "Interaction of individual skyrmions in a nanostructured cubic chiral magnet," Physical Review Letters, vol. 120, no. 19, p. 197203, 2018.

[133] B. M. Ludbrook, B. J. Ruck, and S. Granville, "Perpendicular magnetic anisotropy in $\mathrm{Co}_{2} \mathrm{Fe}_{0.4} \mathrm{Mn}_{0.6} \mathrm{Si}$," Journal of Applied Physics, vol. 120, no. 1, p. 013905, 2016.

[134] B. Ludbrook, B. Ruck, and S. Granville, "Perpendicular magnetic anisotropy in $\mathrm{Co}_{2} \mathrm{MnGa}$ and its anomalous Hall effect," Applied Physics Letters, vol. 110, no. 6, p. 062408, 2017.

[135] J. Hu, Y. Zhang, B. Wei, S. Tu, S. Liu, D. Yu, J.-P. Ansermet, S. Granville, $\mathrm{H}$. Yu, et al., "Anomalous Nernst effect in $\mathrm{Co}_{2} \mathrm{MnGa}$ thin films with perpendicular magnetic anisotropy," Journal of Magnetism and Magnetic Materials, vol. 500, p. 166397, 2020.

[136] J. Hu, T. Butler, M. A. Cabero Z, H. Wang, B. Wei, S. Tu, C. Guo, C. Wan, X. Han, S. Liu, et al., "Regulating the anomalous Hall and Nernst effects in Heusler-based trilayers," Applied Physics Letters, vol. 117, no. 6, p. 062405, 2020.

[137] Z. Qin, Y. Wang, S. Zhu, C. Jin, J. Fu, Q. Liu, and J. Cao, "Stabilization and reversal of skyrmion lattice in $\mathrm{Ta} / \mathrm{CoFeB} / \mathrm{MgO}$ multilayers," 
ACS Applied Materials \& Interfaces, vol. 10, no. 42, pp. 36556-36563, 2018.

[138] M. Hervé, B. Dupé, R. Lopes, M. Böttcher, M. D. Martins, T. Balashov, L. Gerhard, J. Sinova, and W. Wulfhekel, "Stabilizing spin spirals and isolated skyrmions at low magnetic field exploiting vanishing magnetic anisotropy," Nature Communications, vol. 9, no. 1, p. 1015, 2018.

[139] M. Schott, A. Bernand-Mantel, L. Ranno, S. Pizzini, J. Vogel, H. Béa, C. Baraduc, S. Auffret, G. Gaudin, and D. Givord, "The skyrmion switch: turning magnetic skyrmion bubbles on and off with an electric field," Nano Letters, vol. 17, no. 5, pp. 3006-3012, 2017.

[140] T. Srivastava, M. Schott, R. Juge, V. Krizakova, M. Belmeguenai, Y. Roussigné, A. Bernand-Mantel, L. Ranno, S. Pizzini, S.-M. Chérif, et al., "Large-voltage tuning of Dzyaloshinskii-Moriya interactions: A route toward dynamic control of skyrmion chirality," Nano Letters, vol. 18, no. 8, pp. 4871-4877, 2018.

[141] C. Ma, X. Zhang, J. Xia, M. Ezawa, W. Jiang, T. Ono, S. Piramanayagam, A. Morisako, Y. Zhou, and X. Liu, "Electric fieldinduced creation and directional motion of domain walls and skyrmion bubbles," Nano Letters, vol. 19, no. 1, pp. 353-361, 2018.

[142] D. Bhattacharya, S. A. Razavi, H. Wu, B. Dai, K. L. Wang, and J. Atulasimha, "Creation and annihilation of non-volatile fixed magnetic skyrmions using voltage control of magnetic anisotropy," Nature Electronics, vol. 3, no. 9, pp. 539-545, 2020.

[143] U. Bauer, L. Yao, A. J. Tan, P. Agrawal, S. Emori, H. L. Tuller, S. Van Dijken, and G. S. Beach, "Magneto-ionic control of interfacial magnetism," Nature Materials, vol. 14, no. 2, pp. 174-181, 2015. 
[144] S. Zhao, Z. Zhou, B. Peng, M. Zhu, M. Feng, Q. Yang, Y. Yan, W. Ren, Z.-G. Ye, Y. Liu, et al., "Quantitative determination on ionic-liquidgating control of interfacial magnetism," Advanced Materials, vol. 29, no. 17, p. 1606478, 2017.

[145] Y. Zhou and S. Ramanathan, "Relaxation dynamics of ionic liquid$\mathrm{VO}_{2}$ interfaces and influence in electric double-layer transistors," Journal of Applied Physics, vol. 111, no. 8, p. 084508, 2012.

[146] C. Schlenker, S. S. Parkin, J. Scott, and K. Howard, "Magnetic disorder in the exchange bias bilayered FeNi-FeMn system," Journal of Magnetism and Magnetic Materials, vol. 54, pp. 801-802, 1986.

[147] K. Kim, A. Gossmann, and N. Winograd, "X-ray photoelectron spectroscopic studies of palladium oxides and the palladium-oxygen electrode," Analytical Chemistry, vol. 46, no. 2, pp. 197-200, 1974.

[148] W. Moddeman, W. Bowling, D. Carter, and D. Grove, "XPS surface and bulk studies of heat treated palladium in the presence of hydrogen at $150^{\circ} \mathrm{C}$," Surface and Interface Analysis, vol. 11, no. 6-7, pp. 317326, 1988.

[149] J. Zemek, S. Hucek, A. Jablonski, and I. Tilinin, "Photoelectron escape depth," Journal of Electron Spectroscopy and Related Phenomena, vol. 76, pp. 443-447, 1995.

[150] N. Lu, P. Zhang, Q. Zhang, R. Qiao, Q. He, H.-B. Li, Y. Wang, J. Guo, D. Zhang, Z. Duan, et al., "Electric-field control of tri-state phase transformation with a selective dual-ion switch," Nature, vol. 546, no. 7656, pp. 124-128, 2017.

[151] H. Ohta, Y. Sato, T. Kato, S. Kim, K. Nomura, Y. Ikuhara, and H. Hosono, "Field-induced water electrolysis switches an oxide semiconductor from an insulator to a metal," Nature Communications, vol. 1, no. 1, p. 118, 2010. 
[152] J. Jeong, N. Aetukuri, T. Graf, T. D. Schladt, M. G. Samant, and S. S. Parkin, "Suppression of metal-insulator transition in $\mathrm{VO}_{2}$ by electric field-induced oxygen vacancy formation," Science, vol. 339, no. 6126, pp. 1402-1405, 2013.

[153] A. J. Tan, M. Huang, C. O. Avci, F. Büttner, M. Mann, W. Hu, C. Mazzoli, S. Wilkins, H. L. Tuller, and G. S. Beach, "Magneto-ionic control of magnetism using a solid-state proton pump," Nature Materials, vol. 18, no. 1, pp. 35-41, 2019.

[154] H. Yuan, H. Shimotani, A. Tsukazaki, A. Ohtomo, M. Kawasaki, and Y. Iwasa, "High-density carrier accumulation in $\mathrm{ZnO}$ field-effect transistors gated by electric double layers of ionic liquids," Advanced Functional Materials, vol. 19, no. 7, pp. 1046-1053, 2009.

[155] H. C. Koo, S. B. Kim, H. Kim, T.-E. Park, J. W. Choi, K.-W. Kim, G. Go, J. H. Oh, D.-K. Lee, E.-S. Park, et al., "Rashba effect in functional spintronic devices," Advanced Materials, p. 2002117, 2020.

[156] W. Wang, T. Wang, V. P. Amin, Y. Wang, A. Radhakrishnan, A. Davidson, S. R. Allen, T. J. Silva, H. Ohldag, D. Balzar, et al., "Anomalous spin-orbit torques in magnetic single-layer films," $\mathrm{Na}$ ture Nanotechnology, vol. 14, no. 9, pp. 819-824, 2019.

[157] S. Holmes and M. Pepper, "Magnetic and electrical properties of $\mathrm{Co}_{2} \mathrm{MnGa}$ grown on GaAs (001)," Applied Physics Letters, vol. 81, no. 9, pp. 1651-1653, 2002.

[158] C. Yu, M. J. Pechan, D. Carr, and C. J. Palmstrøm, "Ferromagnetic resonance in the stripe domain state: A study in $\mathrm{Co}_{2} \mathrm{MnGa}(001), "$ Journal of Applied Physics, vol. 99, no. 8, p. 08J109, 2006.

[159] Y. Kudryavtsev, V. Oksenenko, Y. Lee, Y. Hyun, J. Kim, J. Park, S. Park, and J. Dubowik, "Evolution of the magnetic properties of 
$\mathrm{Co}_{2} \mathrm{MnGa}$ Heusler alloy films: From amorphous to ordered films," Physical Review B, vol. 76, no. 2, p. 024430, 2007.

[160] M. Kolbe, S. Chadov, E. A. Jorge, G. Schönhense, C. Felser, H.-J. Elmers, M. Kläui, and M. Jourdan, “Test of band structure calculations for Heusler compounds by spin-resolved photoemission spectroscopy," Physical Review B, vol. 86, no. 2, p. 024422, 2012.

[161] H. Reichlova, R. Schlitz, S. Beckert, P. Swekis, A. Markou, Y.-C. Chen, D. Kriegner, S. Fabretti, G. Hyeon Park, A. Niemann, et al., "Large anomalous Nernst effect in thin films of the Weyl semimetal $\mathrm{Co}_{2} \mathrm{MnGa}$," Applied Physics Letters, vol. 113, no. 21, p. 212405, 2018.

[162] G.-H. Park, H. Reichlova, R. Schlitz, M. Lammel, A. Markou, P. Swekis, P. Ritzinger, D. Kriegner, J. Noky, J. Gayles, et al., “Thickness dependence of the anomalous Nernst effect and the Mott relation of Weyl semimetal $\mathrm{Co}_{2} \mathrm{MnGa}$," Physical Review B, vol. 101, no. 6, p. $060406,2020$.

[163] A. Markou, D. Kriegner, J. Gayles, L. Zhang, Y.-C. Chen, B. Ernst, Y.-H. Lai, W. Schnelle, Y.-H. Chu, Y. Sun, et al., “Thickness dependence of the anomalous Hall effect in thin films of the topological semimetal $\mathrm{Co}_{2} \mathrm{MnGa}$," Physical Review B, vol. 100, no. 5, p. 054422, 2019.

[164] Q. Wang, Z. Wen, T. Kubota, T. Seki, and K. Takanashi, “Structuralorder dependence of anomalous Hall effect in $\mathrm{Co}_{2} \mathrm{MnGa}$ topological semimetal thin films," Applied Physics Letters, vol. 115, no. 25, p. 252401, 2019.

[165] J. Kübler, Theory of itinerant electron magnetism, vol. 106. Oxford University Press, 2017.

[166] L. Zhu and J. Zhao, "Anomalous resistivity upturn in epitaxial L2 ${ }_{1}$ $\mathrm{Co}_{2} \mathrm{MnAl}$ films," Scientific Reports, vol. 7, no. 1, pp. 1-7, 2017. 
[167] Y. Liu, L. Shelford, V. Kruglyak, R. Hicken, Y. Sakuraba, M. Oogane, and Y. Ando, "Optically induced magnetization dynamics and variation of damping parameter in epitaxial $\mathrm{Co}_{2} \mathrm{MnSi}$ Heusler alloy films," Physical Review B, vol. 81, no. 9, p. 094402, 2010.

[168] T. Suzuki, R. Chisnell, A. Devarakonda, Y.-T. Liu, W. Feng, D. Xiao, J. W. Lynn, and J. Checkelsky, "Large anomalous Hall effect in a halfHeusler antiferromagnet," Nature Physics, vol. 12, no. 12, pp. 1119 1123, 2016.

[169] R. Singha, S. Roy, A. Pariari, B. Satpati, and P. Mandal, "Magnetotransport properties and giant anomalous Hall angle in the half-Heusler compound TbPtBi," Physical Review B, vol. 99, no. 3, p. 035110, 2019.

[170] S. Tong, X. Zhao, D. Wei, and J. Zhao, "Low-temperature resistivity anomaly and weak spin disorder in $\mathrm{Co}_{2} \mathrm{MnGa}$ epitaxial thin films," Physical Review B, vol. 101, no. 18, p. 184434, 2020.

[171] S. Onoda, N. Sugimoto, and N. Nagaosa, "Intrinsic versus extrinsic anomalous Hall effect in ferromagnets," Physical Review Letters, vol. 97, no. 12, p. 126602, 2006.

[172] M. Hirschberger, S. Kushwaha, Z. Wang, Q. Gibson, S. Liang, C. A. Belvin, B. A. Bernevig, R. J. Cava, and N. P. Ong, "The chiral anomaly and thermopower of Weyl fermions in the half-Heusler GdPtBi," Nature Materials, vol. 15, no. 11, pp. 1161-1165, 2016.

[173] J. Xiong, S. K. Kushwaha, T. Liang, J. W. Krizan, M. Hirschberger, W. Wang, R. J. Cava, and N. P. Ong, "Evidence for the chiral anomaly in the Dirac semimetal $\mathrm{Na}_{3} \mathrm{Bi}$," Science, vol. 350, no. 6259, pp. 413416, 2015. 
[174] R. Gopal, Y. Singh, et al., "Observation of planar Hall effect in the ferromagnetic Weyl semimetal $\mathrm{Co}_{3} \mathrm{Sn}_{2} \mathrm{~S}_{2}$," Journal of Magnetism and Magnetic Materials, vol. 502, p. 166547, 2020.

[175] Y.-C. Chen, M. Yen, Y.-H. Lai, A. Markou, L. Zhang, Y.-Y. Chin, H.J. Lin, C.-T. Chen, C. Felser, and Y.-H. Chu, "Heteroepitaxy of Cobased Heusler compound/muscovite for flexible spintronics," ACS Applied Materials E Interfaces, vol. 11, no. 38, pp. 35162-35168, 2019.

[176] D. Xiao, M.-C. Chang, and Q. Niu, "Berry phase effects on electronic properties," Reviews of Modern Physics, vol. 82, no. 3, p. 1959, 2010.

[177] K. Sumida, Y. Sakuraba, K. Masuda, T. Kono, M. Kakoki, K. Goto, W. Zhou, K. Miyamoto, Y. Miura, T. Okuda, et al., "Spin-polarized Weyl cones and giant anomalous Nernst effect in ferromagnetic Heusler films," Communications Materials, vol. 1, no. 1, pp. 1-9, 2020.

[178] C. Schindler, J. Noky, M. Schmidt, C. Felser, J. Wosnitza, and J. Gooth, "Effect of uniaxial stress on the electronic band structure of NbP," Physical Review B, vol. 102, no. 3, p. 035132, 2020.

[179] A. Ayuela, J. Enkovaara, K. Ullakko, and R. Nieminen, "Structural properties of magnetic Heusler alloys," Journal of Physics: Condensed Matter, vol. 11, no. 8, p. 2017, 1999.

[180] N. Thiyagarajah, Y.-C. Lau, D. Betto, K. Borisov, J. Coey, P. Stamenov, and K. Rode, "Giant spontaneous Hall effect in zero-moment $\mathrm{Mn}_{2} \mathrm{Ru}_{x} \mathrm{Ga}$," Applied Physics Letters, vol. 106, no. 12, p. 122402, 2015.

[181] J. Hu, B. Ernst, S. Tu, M. Kuveždić, A. Hamzić, E. Tafra, M. Basletić, Y. Zhang, A. Markou, C. Felser, et al., "Anomalous Hall and Nernst effects in $\mathrm{Co}_{2} \mathrm{TiSn}$ and $\mathrm{Co}_{2} \mathrm{Ti}_{0.6} \mathrm{~V}_{0.4} \mathrm{Sn}$ Heusler thin films," Physical Review Applied, vol. 10, no. 4, p. 044037, 2018. 
[182] T. McGuire, R. Gambino, and R. Taylor, "Hall effect in amorphous thin-film magnetic alloys," Journal of Applied Physics, vol. 48, no. 7, pp. 2965-2970, 1977.

[183] Y. Zhang, N. Sun, W. Che, R. Shan, and Z. Zhu, "Origin of enhanced anomalous Hall effect in ultrathin Pt/permalloy bilayers," AIP Advances, vol. 6, no. 2, p. 025214, 2016.

[184] J. Moritz, B. Rodmacq, S. Auffret, and B. Dieny, "Extraordinary Hall effect in thin magnetic films and its potential for sensors, memories and magnetic logic applications," Journal of Physics D: Applied Physics, vol. 41, no. 13, p. 135001, 2008.

[185] C. Sürgers, G. Fischer, P. Winkel, and H. v. Löhneysen, "Magnetotransport in ferromagnetic $\mathrm{Mn}_{5} \mathrm{Ge}_{3}, \mathrm{Mn}_{5} \mathrm{Ge}_{3} \mathrm{C}_{0} .8$, and $\mathrm{Mn}_{5} \mathrm{Si}_{3} \mathrm{C}_{0.8}$ thin films," Physical Review B, vol. 90, no. 10, p. 104421, 2014.

[186] E. Vilanova Vidal, G. Stryganyuk, H. Schneider, C. Felser, and G. Jakob, "Exploring $\mathrm{Co}_{2} \mathrm{MnAl}$ Heusler compound for anomalous Hall effect sensors," Applied Physics Letters, vol. 99, no. 13, p. 132509, 2011.

[187] K. Kim, J. Seo, E. Lee, K.-T. Ko, B. Kim, B. G. Jang, J. M. Ok, J. Lee, Y. J. Jo, W. Kang, et al., "Large anomalous Hall current induced by topological nodal lines in a ferromagnetic van der Waals semimetal," $\mathrm{Na}-$ ture Materials, vol. 17, no. 9, pp. 794-799, 2018.

[188] W. Jiang, X. Zhang, G. Yu, W. Zhang, X. Wang, M. B. Jungfleisch, J. E. Pearson, X. Cheng, O. Heinonen, K. L. Wang, et al., "Direct observation of the skyrmion Hall effect," Nature Physics, vol. 13, no. 2, pp. 162-169, 2017. 


\section{List of Publications}

1. Y. Zhang, Y. Yin, G. Dubuis, T. Butler, N. V. Medhekar, S. Granville, Berry curvature origin of the thickness-dependent anomalous Hall effect in a ferromagnetic Weyl semimetal, npj Quantum Materials, 6, 17 (2021).

2. Y. Zhang, G. Dubuis, C. Doyle, T. Butler, S. Granville, Fractal analysis of skyrmions generated by field-assisted fine-tuning of magnetic anisotropy, Physical Review Applied, 15, 014020 (2021).

3. Y. Zhang, G. Dubuis, T. Butler, S. Granville, Non-volatile and volatile skyrmion generation engineered by ionic liquid gating in ultrathin films, Physical Review Applied, 16, 014030 (2021).

4. Y. Zhang, G. Dubuis, T. Butler, S. Kaltenberg, E. Trewick, S. Granville, Ionic liquid gated ambipolar transport in perpendicularly magnetized Heusler ultrathin films, (in preparation)

5. L. Leiva, S. Granville, Y. Zhang, S. Dushenko, E. Shigematsu, T. Shinjo, R. Ohshima, Y. Ando, and M. Shiraishi, Colossal enhancement of spin caloritronic unidirectional magnetoresistance in a Weyl ferromagnet at room temperature, (Under review)

6. L. Leiva, S. Granville, Y. Zhang, S. Dushenko, E. Shigematsu, T. Shinjo, R. Ohshima, Y. Ando, and M. Shiraishi, Giant spin Hall angle in the Heusler alloy Weyl ferromagnet $\mathrm{Co}_{2} \mathrm{MnGa}$, Physical Review B, 103, L041114 (2021).

7. J. F. Hu, Y. Zhang(co-1 ${ }^{s t}$ ), M. C. Cabero, B. H. Wei, S. Tu, S. Liu, D. P. Yu, J.-P. Ansermet, S. Granville, H. M. Yu, Anomalous Nernst effect in $\mathrm{Co}_{2} \mathrm{MnGa}$ thin films with perpendicular magnetic anisotropy, Journal of Magnetism and Magnetic Materials, 500, 166397 (2020). 


\section{List of Figures}

1.1 Typical GMR curve, the magnetoresistance as a function magnetic field. . . . . . . . . . . . . . 5

1.2 Schematic of the mechanism of GMR. FM: ferromagnetic layer (arrows indicate the direction of magnetization), NM: non-magnetic layer. Electrons with spin up and down scatter differently in the multilayer. The bottom shows the equivalent circuit diagrams for the total resistances. $R_{\downarrow \downarrow}\left(R_{\uparrow \downarrow}\right)$ denotes the resistance for electrons with spin aligned with the same(different) direction of the magnetization of FM layer. . 6

1.3 Schematic of the mechanism of spin transfer torque. . . . . . 7

1.4 Schematic of magnetic DW racetrack memory [30]. . . . . . . 8

1.5 Magnetic moment associated with orbital motion (a) and spin (b) of an electron. . . . . . . . . . . . . . . . . . . 10

1.6 (a) Spontaneous magnetization below $T_{\mathrm{c}}$. (b) The random behaviour of magnetization above $T_{\mathrm{c}}$. (c)-(d) The alignment of magnetic domains without or with magnetic field, respectively. (e) Hysteresis loop. (f) The magnetization as a function of temperature. . . . . . . . . . . . . 11

1.7 Schematic of demagnetizing field. . . . . . . . . . . . 14

1.8 Typical curve about $K_{\text {eff }} \cdot t$ versus the thickness of ferromagnetic layer in multilayers. The flat plates and arrows denote the thin film and the direction of magnetization, respectively. ..................... 15 
1.9 Schematic of DMI between two neighbouring spins $\mathbf{S}_{i}$ and $\mathbf{S}_{j}$ at the interface between ferromagnet and heavy metal layer. The DMI vector $\mathbf{D}_{i j}$ is along the $y$ axis direction. . . . . 17

1.10 (a) A single domain, (b) A double-domain and (c) a multidomain. The arrow and dash lines represent magnetic moment and demagnetizing field, respectively. . . . . . . . . 18

1.11 Schematic view of magnetic moments rotation in a Bloch wall (a) and Néel wall (b) between two domains with a opposite magnetized direction. The dashed arrows show the axis of rotation of magnetization. The thickness direction is along the $z$ axis. . . . . . . . . . . . . . . 19

1.12 Cross section of a Bloch and Néel wall in thin films with different thickness $t$ and wall width $\Delta$. The arrows represent the projection on the cross section plane. . . . . . . . 20

1.13 The spin rotation of chiral Néel domain walls. . . . . . . . . 21

1.14 (a) Spin texture of skyrmions. (b) The spin profile from the cross-section of Néel-type skyrmion and its angular progression which is the same as the non-trivial DWs. (c) The angular progression of the trivial DWs. . . . . . . . . 22

1.15 Skyrmion-based magnetic racetrack memory. The presence of a skyrmion code data bit 1 and the absence of a skyrmion code data bit 0 . Skyrmion driven by a current pulse. The red dot denotes skyrmion. . . . . . . . . . . . . . 23

1.16 Periodic table of the elements. The Heusler compounds can be formed by combination of the different elements for $X$ (red), $Y$ (blue) and Z (purple) [54].

1.17 Crystal structures. (a) half Heusler alloys $C 1_{b}$ structure, (b) full Heusler alloys $L 2_{1}$ structure, (c) disordered $B 2$ and (d) A2 structure. [56] . . . . . . . . . . . . . . 25 
1.18 Schematic illustration of density of states for ordinary ferromagnet (a), half-metallic ferromagnet (b) and spin gapless ferromagnet (c). Arrows indicate the majority $(\uparrow)$ and minority $(\downarrow)$ states. . . . . . . . . . . . . . 26

1.19 Calculated total spin magnetic moments for Heusler alloys. The dashed line represents the Slater-Pauling behaviour [62]. 27

1.20 (a) The schematic of energy spectrum of a Weyl semimetal. The conduction and valence bands touch at the Weyl nodes with Fermi arcs. $k_{\mathrm{z}}$ is the wave vector. (b) Parallel transport of a vector along a closed path on a sphere. . . . . . . . . . 29

2.1 Schematic of thin film growth process with magnetron sputtering. . . . . . . . . . . . . . . . . . 34

2.2 (a) CMS-18 magnetron sputtering system (Robinson Research Institute) and (b) flux of plasma. . . . . . . . . . . . . . 35

2.3 The processes of device fabrication. (a) Thin film deposition; (b) Spin coating photoresist; (c) Exposing and developing photoresist into the device pattern; (d) Ar milling; (e) Final device; (f) An example device measured by optical microscopy. . . . . . . . . . . . . . . . 36

2.4 (a) Schematic of laser writer. "I am a robot" is an example of the designed pattern structure. (b) Microtech Lw405 model (Callaghan Innovation, Wellington). . . . . . . . . . . 37

2.5 Ion milling (Robinson Research Institute). . . . . . . . . . . . 38

2.6 (a) Schematic of electric double layer (b) The decay of the electrical potential (red line) in the electric double layer. . . 39

2.7 (a) Schematic of XRD and (b) rocking curve geometry; (c) Bruker D8 XRD system . . . . . . . . . . . . . . . . 40

2.8 (a) Schematic of XRR geometry and (b) an experimental example of $20 \mathrm{~nm} \mathrm{Co}_{2} \mathrm{MnGa}$ thin film. . . . . . . . . . . . . 41

2.9 (a) Schematic of EDX (b) an experimental spectrum of $\mathrm{CO}_{2} \mathrm{MnGa}$ thin film on $\mathrm{MgO}$ substrate. . . . . . . . . . . . 43 
2.10 (a) Schematic of AFM and (b) force curve as a function of $z$ distance. . . . . . . . . . . . . . . . 44

2.11 (a) Schematic of XPS principle. . . . . . . . . . . . . . 45

2.12 (a) Four-wire resistance geometry and (b) Van der Pauw geometry. ................... 46

2.13 Top: horizontal rotator, and bottom: resistivity pucks with different function [95].

2.14 (a) Schematic of AHE. Mechanism of AHE, (b) intrinsic deflection, (c) side jump, and (d) skew scattering. . . . . . . . . 48

2.15 (a) Parallel and (b) perpendicular geometry. . . . . . . . . . . 48

2.16 Schematic of the RSO measurement of the SQUID magnetometer [100]. The W-shape curve represents the signal for an ideal magnetic dipole detected by the SQUID coils as a function of position. . . . . . . . . . . . . . 50

2.17 (a) Schematic of MOKE. (b) The change of electric field of polarized light after propagating into medium 2. $E_{1(2)}^{(\mathrm{i})}$ and $E_{1(2)}^{(\mathrm{r})}$ represent the incident and reflected light, respectively, in medium 1 or medium 2. $E_{1 \mathrm{~s}}^{(\mathrm{i})}$ and $E_{1 \mathrm{p}}^{(\mathrm{i})}$ represent the direction of electric field of incident light perpendicular and parallel to the plane of incidence, respectively. $k$ is the wave vector. . . . . . . . . . . . . . . . . . . . 51

2.18 Schematic of MOKE. A linearly polarized light illuminates a magnetic material, then there is a contrast for those domains with opposite direction of magnetic moments. . . . . . . . . 52

2.19 (a) Longitudinal and (b) polar MOKE configurations. . . . . 53

2.20 (a) Schematic of Stoner-Wohlfarth model for a single domain. (b) Rotation of magnetization from out-of-plane to in-plane direction by sweeping the in-plane magnetic field for $\mathrm{MgO}(1.6 \mathrm{~nm}) / \mathrm{Mn}_{2} \mathrm{CoAl}(2.6 \mathrm{~nm}) / \mathrm{Pd}(3.2 \mathrm{~nm})$ trilayer. . . 54 
2.21 Schematic of magnetic bubble expansion. The gray and white colour denote the direction of magnetization along up and down direction, respectively. . . . . . . . . . . . . . 56

2.22 (a) DW expansion for stack $\mathrm{MgO}(1.6 \mathrm{~nm}) / \mathrm{Mn}_{2} \operatorname{CoAl}(2.6 \mathrm{~nm}) / \operatorname{Pd}(2.2$ $\mathrm{nm})$. DW displacement after applying a pulse of OP field, $\mu_{0} H_{\mathrm{z}}=9.4 \mathrm{mT}$, with $700 \mathrm{~ms}$. (b) DW displacement after applying OP field, $\mu_{0} H_{\mathrm{z}}=9.4 \mathrm{mT}$, with $700 \mathrm{~ms}$ under $\mu_{0} H_{\mathrm{x}}$ $=-35.5 \mathrm{mT}$. (c) DW displacement after applying OP field, $\mu_{0} H_{\mathrm{z}}=9.4 \mathrm{mT}$, with $700 \mathrm{~ms}$ under $\mu_{0} H_{\mathrm{x}}=23.7 \mathrm{mT} . \ldots . .57$

3.1 An experimental EDX spectrum of $100 \mathrm{~nm} \mathrm{Mn}_{2} \mathrm{CoAl}$ thin film on $\mathrm{MgO}$ substrate measured at $25 \mathrm{KV}$. . . . . . . . . 60

3.2 (a) Hysteresis loop of $100 \mathrm{~nm} \mathrm{Mn}_{2} \mathrm{CoAl}$ measured with an in-plane magnetic field at $300 \mathrm{~K}$. The magnetic field swept from $90 \mathrm{mT}$ down to $-90 \mathrm{mT}$. (b) Temperature dependence of magnetization under a $100 \mathrm{mT}$ in-plane magnetic field [68]. The red line is an extrapolation to estimate the Curie temperature by $M(T)=M(0)\left[1-\left(T / T_{\mathrm{c}}\right)^{2}\right]^{1 / 2} \ldots \ldots \ldots 1$

3.3 The atomic force microscopy image of $\mathrm{MgO} / \mathrm{Mn}_{2} \mathrm{CoAl} / \mathrm{Pd}$ ultrathin film for two dimensions (a) and three dimensions (b) $\left(5 \times 5 \mu \mathrm{m}^{2}\right)$ with colour contrast (dark to bright) for the height scale. . . . . . . . . . . . . . . 62

3.4 Hysteresis loop of $\mathrm{MgO}(2.0 \mathrm{~nm}) / \mathrm{Mn}_{2} \operatorname{CoAl}(2.6 \mathrm{~nm}) / \operatorname{Pd}(2.0$ nm) measured at $300 \mathrm{~K} . \ldots \ldots$. . . . . . . . . . 63

3.5 Hysteresis loops of $\mathrm{MgO}(1.6 \mathrm{~nm}) / \mathrm{Mn}_{2} \mathrm{CoAl}(2.6 \mathrm{~nm}) / \mathrm{Pd}(2.2$ $-4.4 \mathrm{~nm}$ ) measured at ambient temperature with a field along the OP direction. . . . . . . . . . . . . . . . . . . 64

3.6 $M_{\mathrm{r}} / M_{\mathrm{s}}, \mu_{0} H_{\mathrm{c}}$ and $K_{\mathrm{eff}}$ as function of $t_{\mathrm{Pd}}$ in $\mathrm{MgO} / \mathrm{Mn}_{2} \mathrm{CoAl} / \mathrm{Pd}$ stacks. ......................... 65 
3.7 Typical magnetic domain images for various $\mathrm{MgO} / \mathrm{Mn}_{2} \mathrm{CoAl} / \mathrm{Pd}$ trilayers at certain magnetic field. $t_{\mathrm{Pd}}$ denotes the thickness of Pd layer. The scale bar corresponds to $80 \mu \mathrm{m}$ in (a) and (b). The scale bar corresponds to $8 \mu \mathrm{m}$ in (c) and (d). . . . 66

4.1 (a) Hysteresis loop of $\mathrm{MgO}(1.6 \mathrm{~nm}) / \mathrm{Mn}_{2} \mathrm{CoAl}(2.6 \mathrm{~nm}) / \mathrm{Pd}(3.2$ $\mathrm{nm})$ ultrathin film under an OP field at ambient temperature, $\sim 294$ K. (b) Rotation of magnetization from OP to IP direction by sweeping the IP field.(c) DW expansion for stack $\mathrm{MgO}(1.6 \mathrm{~nm}) / \mathrm{Mn}_{2} \mathrm{CoAl}(2.6 \mathrm{~nm}) / \mathrm{Pd}(2.2 \mathrm{~nm})$. DW displacement after applying a pulse of OP field, $\mu_{0} H_{\mathrm{z}}=9.4 \mathrm{mT}$, with $700 \mathrm{~ms}$. (d) DW displacement after applying OP field, $\mu_{0} H_{\mathrm{z}}=9.4 \mathrm{mT}$, with $700 \mathrm{~ms}$ under $\mu_{0} H_{\mathrm{x}}=-35.5 \mathrm{mT}$. (e) DW displacement after applying OP field, $\mu_{0} H_{\mathrm{z}}=9.4 \mathrm{mT}$, with $700 \mathrm{~ms}$ under $\mu_{0} H_{\mathrm{x}}=23.7 \mathrm{mT} \ldots \ldots \ldots \ldots \ldots$

4.2 (a)-(i) Field-dependent domain morphology of $\mathrm{MgO}(1.6 \mathrm{~nm})$ $/ \mathrm{Mn}_{2} \mathrm{CoAl}(2.6 \mathrm{~nm}) / \mathrm{Pd}(3.2 \mathrm{~nm})$ at ambient temperature. The black and grey features represent magnetization $+m_{\mathrm{z}}$ and $-m_{z}$, respectively. The scale bar in (a) corresponds to 25 $\mu \mathrm{m}$. (j) Counts of skyrmions as a function of effective diameter extracted from (h). The solid line is a fit with a Gaussian distribution. (k) Magnetic hysteresis loops under an IP field 0, 39.5 and $47.3 \mathrm{mT}$, respectively, by sweeping the OP field. The dash lines represent $\mu_{0} H_{\mathrm{z}}=-1.2,-2$ and $-2.3 \mathrm{mT}$, respectively. (1) The effective magnetic anisotropy constant $K_{\text {eff }}$ as a function of IP field. The star represents experimental value extracted from Figure 4.1(b). . . . . . . . . . . 71 
4.3 (a)-(d) The enlarged domain morphology of $\mathrm{MgO}(1.6 \mathrm{~nm})$ $/ \mathrm{Mn}_{2} \mathrm{CoAl}(2.6 \mathrm{~nm}) / \mathrm{Pd}(3.2 \mathrm{~nm})$ under a combination of OP and IP field at ambient temperature. Skyrmions in the purple (resp. orange) region are formed from labyrinth domains (resp. uniformly saturated magnetic domains). The scale bar in (a) corresponds to $5 \mu \mathrm{m} . \ldots \ldots \ldots$. . . . . . 74

4.4 Typical image of demagnetized magnetic domain. . . . . . . 75

4.5 (a) Typical image of magnetic domain under $\mu_{0} H_{z}=-0.7$ $\mathrm{mT}$ after demagnetizing. Black areas represent the downward domains $d_{\downarrow}$. (b) Domain width as a function of OP field and corresponding fitting by the function $y=a \tanh (b x+c)+$

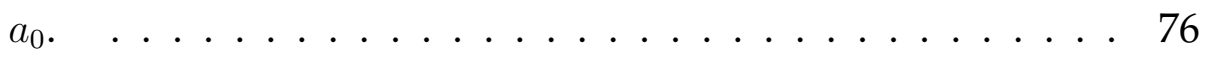

4.6 (a) Original image of magnetic domains. (b) Image after identifying the binary contour pattern. (c)-(d) Image showing the contour of magnetic domain covered by squares with size 8 and 20 pixels, respectively. . . . . . . . . . . . 77

4.7 (a) Log-log plot of the number of elements and the element size for the domain patterns of Figure 4.2(a). The slope of fitting curve represents the fractal dimension. (b) Magnetic phase diagram related to the fractal dimension as a function of IP field under various OP field. The colour bar represents $\gamma$, the total fraction of skyrmions. . . . . . . . . . . . 80

4.8 (a)-(b) The skyrmion density and NND as a function of IP field under $\mu_{0} H_{\mathrm{z}}=-2$ and $-2.3 \mathrm{mT}$, respectively. . . . . . 80

5.1 Schematic of device and gate-dependent magnetic characterization. (a) Schematic of ionic liquid device for $\mathrm{MgO} / \mathrm{Mn}_{2} \mathrm{CoAl} / \mathrm{Pd}$ stacks. (b) Gate dependence of hysteresis loops measured in an out-of-plane magnetic field at ambient temperature. (c)(f) The corresponding magnetic domain images with $\mu_{0} H_{z}=1.0 \mathrm{mT}$ at pristine, $-0.5,-1.5$, and $-2.5 \mathrm{~V}$, respectively. The scale bar in (c) corresponds $10 \mu \mathrm{m} . \ldots \ldots \ldots$. . . . . . . 85 
5.2 Effective magnetic anisotropy, $K_{\text {eff }}$, and DMI coefficient $D$ as a function of gate voltage. The star corresponds to the pristine state.

5.3 Evolution of hysteresis loops and magnetic domains. (a) Hysteresis loop of $\mathrm{MgO}(1.6 \mathrm{~nm}) / \mathrm{Mn}_{2} \mathrm{CoAl}(2.6 \mathrm{~nm}) / \mathrm{Pd}$ $(2.8 \mathrm{~nm})$ trilayer at pristine state. (b)-(d) Hysteresis loops at $-2.5 \mathrm{~V}$ after cycling 21, 27, and 30 times, respectively. (e)(h) The corresponding magnetic domain images, with $\mu_{0} H_{z}$ $=0.66 \mathrm{mT}$, captured from (a)-(d), respectively. The scale bar in (e) corresponds $10 \mu \mathrm{m}$. (i) $\mu_{0} H_{\mathrm{c}}$ as a function of cycle number for 1 and $-2.5 \mathrm{~V}$, respectively. . . . . . . . . 89

5.4 Volatile gating effect for hysteresis loops and magnetic domains. (a)-(b) Hysteresis loops measured at -0.5 and $1.5 \mathrm{~V}$, respectively. (c) Longitudinal hysteresis loop as a function of in-plane magnetic field at $1.5 \mathrm{~V}$. (d) $\mu_{0} H_{\mathrm{c}}$ and $M_{\mathrm{r}} / M_{\mathrm{s}}$ as a function of cycle number at 0 and $1.5 \mathrm{~V}$, respectively. (e)-(f) Hysteresis loops measured at 0 and $1.7 \mathrm{~V}$, respectively. (g)(h) The corresponding magnetic domain images, with $\mu_{0} H_{\mathrm{z}}$ $=1.1 \mathrm{mT}$, captured from $(\mathrm{e})-(\mathrm{f})$, respectively. . . . . . . . 91

5.5 The XPS spectra of trilayers at various gating conditions. The XPS spectra of (a)-(b) pristine sample, (c)-(d) sample after cycling 30 times to skyrmion region, (e)-(f) sample after giving a trigger, $-3 \mathrm{~V} . \ldots \ldots . \ldots 93$

5.6 The XPS spectra of Pd and $\mathrm{O}$ after Ar ions etching 0, 30, 60, 90, and $240 \mathrm{~s}$, respectively, at (a)-(b) pristine state, (c)-(d) cycling 30 times, (e)-(f) $-3 \mathrm{~V}$ trigger. . . . . . . . . . . 96

5.7 The XPS spectra of Co, Mn and Al for pristine state, cycling 30 times, and $-3 \mathrm{~V}$ trigger, respectively, after Ar ions etching $120 \mathrm{~s} . \ldots \ldots \ldots$. . . . . . . . . . . . 96 
5.8 The hysteresis loops of $\mathrm{MgO}(1.6 \mathrm{~nm}) / \mathrm{Mn}_{2} \mathrm{CoAl}(2.6 \mathrm{~nm}) / \mathrm{Pd}(3.2$ $\mathrm{nm})$ at $-2.5 \mathrm{~V}$ after charging 5,28 , and 44 minutes, respec-

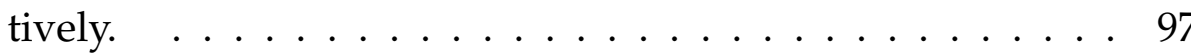

6.1 Crystal structure and surface morphology of $\mathrm{Co}_{2} \mathrm{MnGa}$. (a) $\theta-2 \theta$ scan, the inset is the rocking curve from (004) peak, and (b) in-plane $\varphi$ scans from $-45^{\circ}$ to $315^{\circ}$, for $50 \mathrm{~nm} \mathrm{Co} 2 \mathrm{MnGa}$ thin films. (c) X-ray reflectivity for the $20 \mathrm{~nm} \mathrm{Co}_{2} \mathrm{MnGa}$ thin film, the inset is the atomic force microscopy image $(5 \times 5$ $\mu \mathrm{m}^{2}$ ) with colour contrast (dark to bright) for the height scale corresponds to $3.5 \mathrm{~nm}$. The scale bar corresponds to $1 \mu \mathrm{m} . \ldots \ldots$. . . . . . . . . . . . . . 103

6.2 Magnetic and electric transport measurements for $50 \mathrm{~nm}$ $\mathrm{CO}_{2} \mathrm{MnGa}$. (a) Magnetization measured at $300 \mathrm{~K}$ with the magnetic field along in-plane and out-of-plane direction, respectively. (b) Anomalous Hall effect measurements at 3 and $300 \mathrm{~K}$, respectively. (c) Longitudinal resistivity without field (red) and Hall resistivity cooling down with a $9 \mathrm{~T}$ magnetic field (blue) as a function of temperature from 3 to $300 \mathrm{~K}$. (d) The corresponding conductivity as a function of temperature from 3 to $300 \mathrm{~K}$. . . . . . . . . . . . . . . 104

6.3 (a) Longitudinal resistivity without field as a function of temperature from $3 \mathrm{~K}$ to $300 \mathrm{~K}$ for $\mathrm{Co}_{2} \mathrm{MnGa}(5-50 \mathrm{~nm})$. The inset shows the residual resistivity ratio $\rho_{\mathrm{xx}}(300 \mathrm{~K}) / \rho_{\mathrm{xx}}$ (residual) as a function of thickness. (b) Anomalous Hall resistivity cooling down in $9 \mathrm{~T}$ as a function of temperature from $3 \mathrm{~K}$ to $300 \mathrm{~K}$ for $\mathrm{Co}_{2} \mathrm{MnGa}(5-50 \mathrm{~nm}) . \ldots \ldots$ 
6.4 Transport measurement of the AHA at 9 T. (a) Temperature dependent AHA measured from $3 \mathrm{~K}$ to $300 \mathrm{~K}$ for $50 \mathrm{~nm}$ $\mathrm{Co}_{2} \mathrm{MnGa}$. (b) Thickness dependence of AHA as a function of temperature from $3 \mathrm{~K}$ to $300 \mathrm{~K}$, the inset is the maximum AHA as a function of thickness. (c) $\log \sigma_{\mathrm{xy}}-\sigma_{\mathrm{xx}}$ plot for $\mathrm{Co}_{2} \mathrm{MnGa}$ with thicknesses from $5 \mathrm{~nm}$ to $50 \mathrm{~nm}$. (d)-(e) Contour mapping for thickness dependence of $\sigma_{\mathrm{xx}}$ and $\sigma_{\mathrm{xy}}$ as a function of temperature from $3 \mathrm{~K}$ to $300 \mathrm{~K}$, respectively. 108

6.5 Angular dependence of planar Hall resistivity for various thickness of $\mathrm{Co}_{2} \mathrm{MnGa}$ film. (a) Schematic of measurement geometry. The angle $\theta_{x y}$ represents the angle between the rotating field and $x$-axis when the field rotates from $x$-axis to $y$-axis in the $x y$ plane. Current is along the [100] direction of $\mathrm{Co}_{2} \mathrm{MnGa}$. (b)-(f) Planar Hall resistivity as a function of angle, $\theta_{\mathrm{xy}}$, measured from $300 \mathrm{~K}$ and $3 \mathrm{~K}$ under a magnetic field of $9 \mathrm{~T}$ for 50, 20, 15, 10 and $5 \mathrm{~nm} \mathrm{Co} \mathrm{Cn}_{2} \mathrm{Ma}$ film, respectively. . . . . . . . . . . . . . . 109

6.6 Magnetoresistance (MR) ratio of the $50 \mathrm{~nm} \mathrm{Co}_{2} \mathrm{MnGa}$ film. (a) Schematic of measurement geometry. The angle $\theta_{\mathrm{xz}}$ represents the angle between the rotating field and $x$-axis when the field rotates from $x$-axis to $z$-axis in the $x z$ plane. Current is along the [100] direction of $\mathrm{Co}_{2} \mathrm{MnGa}$. (b) and (c) MR ratio as a function of magnetic field measured at $300 \mathrm{~K}$ and $3 \mathrm{~K}$ with various angles, $\theta_{\mathrm{xz}}$, respectively. . . . . . . . . . 110

6.7 (a)-(d) show the calculated averaged-integral of $z$ component Berry curvature $\left(\Omega_{\mathrm{z}}\right)$ of occupied bands from minority and majority spin electrons for $\mathrm{Co}_{2} \mathrm{MnGa}$ thin films of 4.9 $\mathrm{nm}$ and $50.1 \mathrm{~nm}$. (e) and (f) show a comparison of $\Omega_{\mathrm{z}}$ along $\Gamma$-M line for $\mathrm{Co}_{2} \mathrm{MnGa}$ thin films of 4.9, 15.0, 20.0, and 50.1

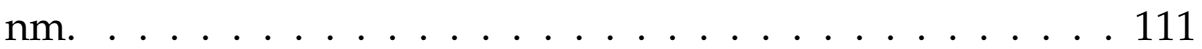


$6.8 \sigma_{\mathrm{xy}}$-dependent AHA of this result in comparison with previously reported results for other thin films. . . . . . . . . 114 


\section{List of Abbreviations}

ACW anticlockwise. 20

AFM atomic force microscopy. 40

AHA anomalous Hall angle. iii

AHE anomalous Hall effect. 29

ASOT anomalous spin-orbit torque. 99

CW clockwise. 20

DMI Dzyaloshinskii-Moriya interaction. 2

DOS density of states. 25

DW domain wall. 7

EDL electric double layer. 39

EDX scanning electron spectroscopy. 40

FM ferromagnet. 4

FWHM full width at half maximum. 41

GMR giant magnetoresistance. 4 
HDD hard disk drives. 5

ILG Ionic liquid gating. 39

IP in-plane. 55

MOKE magneto-optical Kerr effect. 45

MRAM magnetoresistive random-access memory. 1

MTJ magnetic tunnel junction. 2

NND nearest neighbour distance. 57

OP out-of-plane. 55

PMA perpendicular magnetic anisotropy. 2

PPMS physical properties measurement system. 45

PVD physical vapour deposition. 34

RAM random-access memory. 1

RSO reciprocating sample option. 49

SEM scanning electron microscopy. 40

SGS spin gapless semiconductors. 28

SOT spin-orbit torque. 7

SQUID superconducting quantum interference device. 45

STT spin transfer torque. 7

TMR tunneling magnetoresistance. 5 
WSM weyl semimetal. 28

XPS x-ray photoelectron spectroscopy. 40

XRD x-ray diffraction. 40

XRR x-ray reflectivity. 40 